\author{
Aus der ehem. Abteilung \\ Allgemeine Hygiene und Umweltmedizin \\ (Prof. Dr. med. H. Dunkelberg) \\ im Zentrum \\ Arbeits-, Sozial-, Umweltmedizin und Dermatologie, Venerologie und \\ Allergologie \\ der Medizinischen Fakultät der Universität Göttingen
}

\title{
Prüfung eines Testsystems zur mikrobiologischen Barrierewirksamkeit von Sterilgutverpackungen (Papier-Folien- Verbundverpackungen) nach Transport und Lagerung
}

\author{
INAUGURAL - DISSERTATION \\ zur Erlangung des Doktorgrades \\ für Zahnheilkunde \\ der Medizinischen Fakultät \\ der Georg-August-Universität zu Göttingen
}

vorgelegt von

Katharina Niemeyer (geb. Borgolte)

aus

Holzminden

Göttingen 2013 
Dekan: Prof. Dr. rer. nat. H. K. Kroemer

I. Berichterstatter: Prof. Dr. med. H. Dunkelberg

II. Berichterstatter: Prof. Dr. med. U. Reichard

III. Berichterstatter: Prof. Dr. med. dent. R. Mausberg

Tag der mündlichen Prüfung: 30. Juni 2014 


\section{Inhaltsverzeichnis}

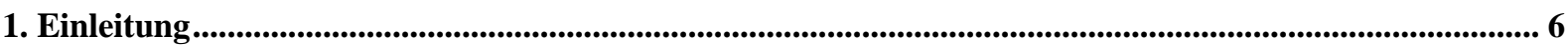

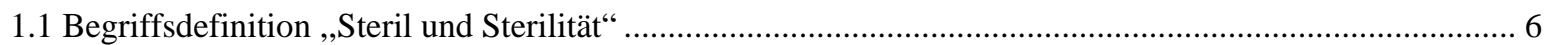

1.2 Fokussierung der Problematik „,Sterilität“ auf die Barrierewirkung der Verpackung................................... 7

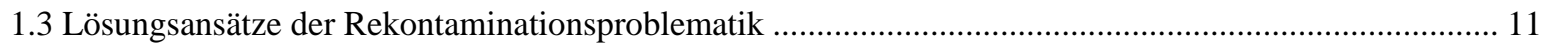

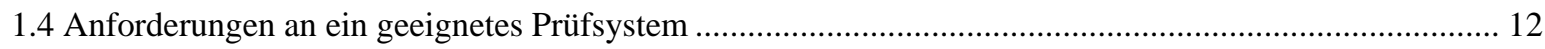

1.5 Darstellung bereits getesteter Verpackungssysteme............................................................................. 13

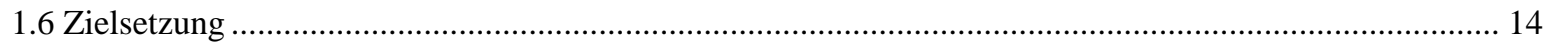

1.7 „Worst- Case- Szenario“ für die zu prüfende Testeinheit: Stand der Ergebnisse zu Beginn der hier

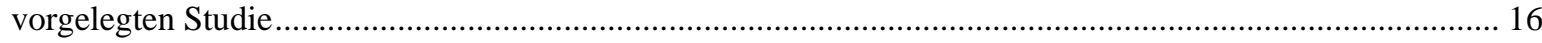

2. Material und methodisches Konzept ................................................................................................................ 20

2.1 Materialien zur Herstellung.............................................................................................................. 20

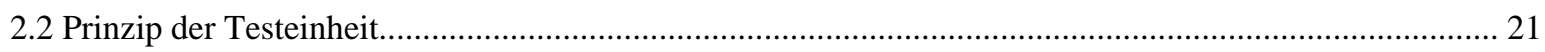

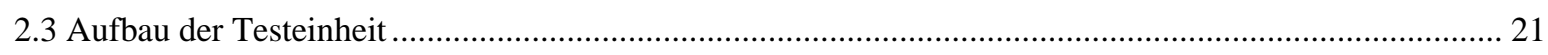

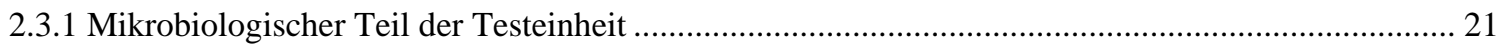

2.3.2 Aktivierungsteil der Testeinheit ................................................................................................... 23

2.4 Handhabung und Anwendung der Testeinheit ...................................................................................... 25

2.5 Kritische Begutachtung der produzierten Testeinheit ................................................................................ 25

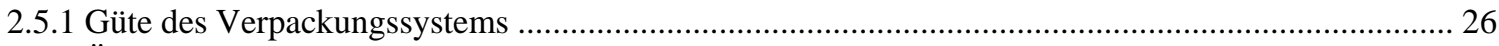

2.5.2 Überprüfung des Aktivierungsteils ................................................................................................... 26

2.5.3 Überprüfung der Anzüchtbarkeit verschiedener Bakterienspezies durch die verwendeten

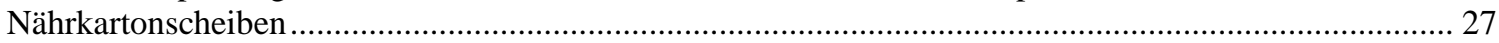

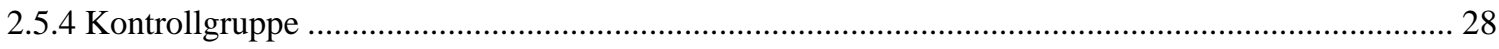

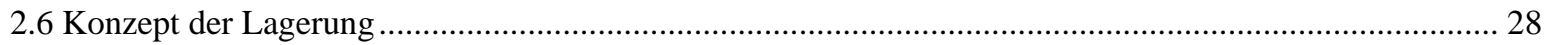

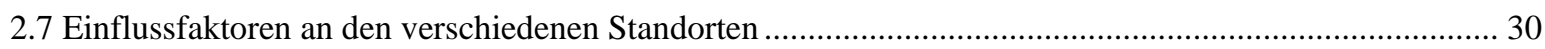

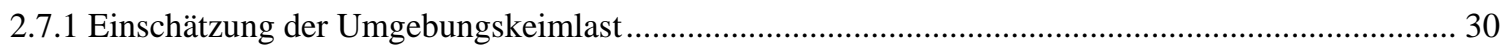

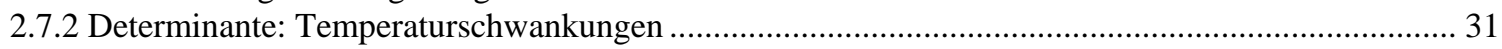

2.7.3 Determinante: atmosphärische Druckschwankungen .................................................................... 32

2.8 Berechnung der Volumenströme ins Verpackungsinnere bei bekannten Druck- und Temperaturverhältnissen 
2.8.1 Berechnung des druckabhängigen Volumenstroms durch die Papierseite der Testeinheit

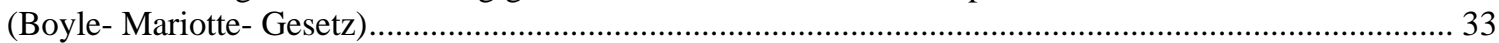

2.8.2 Berechnung der Volumenänderung in Abhängigkeit von der Temperaturdifferenz bei bekanntem

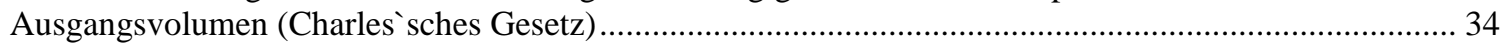

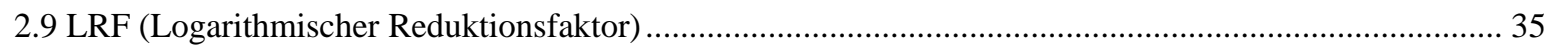

3.1 Auswertung der in den Vorversuchen 2006 ermittelten Rohdaten - Lagerung der Testeinheiten in den Kornhäusern (Dransfeld - Scharzfeld - Obernjesa)

3.1.1 Determinante: atmosphärische Druckänderung.....

3.1.1.1 Einfluss von Luftdruckänderungen auf die gelagerten Testeinheiten in den Kornhäusern

(Dransfeld, Obernjesa, Scharzfeld) und die Kontrollgruppe 1 (2006)....

3.1.1.2 Berechnung des druckabhängigen Volumenstroms durch die Papierseite der Testeinheit ......... 39

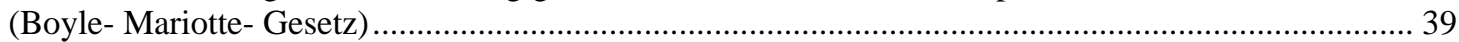

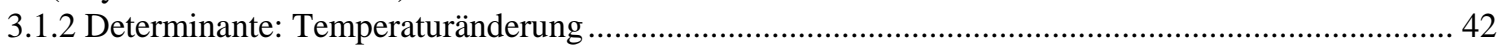

3.1.2.1 Volumenberechnung für die in Dransfeld, Obernjesa und Scharzfeld gelagerten Testeinheiten 42

3.1.3 Kalkulation der mikrobiologischen Beanspruchung der Testeinheiten an den verschiedenen Standorten.....

3.1.4 Berechnung des LRF für die in Obernjesa gelagerten Sterilgutverpackungen

3.2 Auswertung des Expositionsversuchs der Testeinheiten unter realitätsnahen Lagerungsbedingungen im medizinischen Betrieb

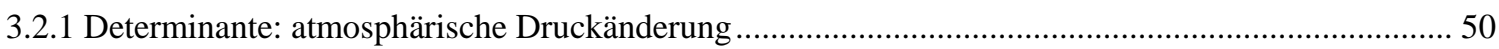

3.2.1.1 Berechnung des druckabhängigen Volumenstroms durch die Papierseite der Testeinheit ......... 51

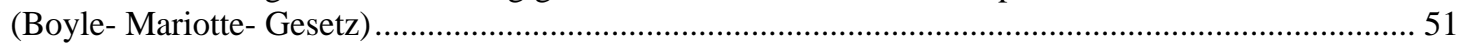

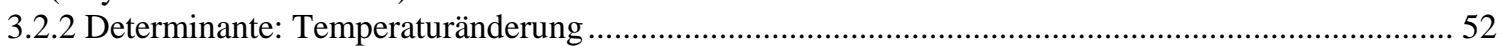

3.2.2.1 Berechnung der Volumenänderung in Abhängigkeit von der Temperaturänderung bei

bekanntem Ausgangsvolumen (Charles`sches Gesetz).................................................................. 52

3.3 Kalkulation der mikrobiologischen Beanspruchung der Testeinheiten an den verschiedenen Standorten. 54

3.3.1 Vergleich der Rekontaminationsrate (\%) und der mikrobiologischen Beanspruchung der Testeinheiten unter den verschiedenen Expositionsbedingungen:.......................................................... 56

Kornhäuser (Dransfeld, Scharzfeld, Obernjesa) und medizinische Versorgungseinrichtungen ( Bad

Rothenfelde, Oldenburg, Institut für Hygiene und Umweltmedizin Göttingen)..... 56

3.4 Berechnung der Rekontaminationswahrscheinlichkeit für die in Bad Rothenfelde und Oldenburg gelagerten Testeinheiten unter Berücksichtigung der anhand der Vorversuche 2006 (Kornhäuser) ermittelten Barrierewirkung

3.5 Aufrechterhaltung der Sterilität unter Berücksichtigung des Sterilitätssicherheitswertes -..... 61 sterility assurance level (SAL) 
4.5 Zusammenhang zwischen Verpackungsdesign und Verpackungsqualität .......................................... 72

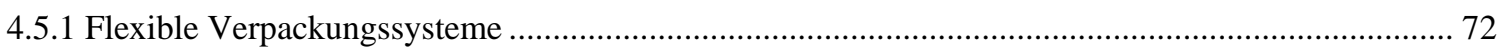

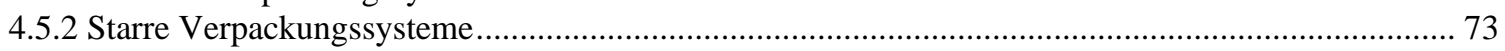

4.6 Einfluss der mikrobiologischen Beanspruchung auf die Wahrung der Sterilität bei verschiedenen

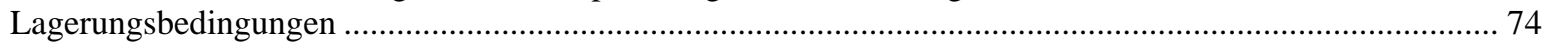

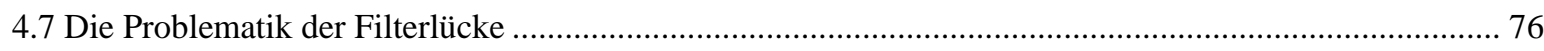

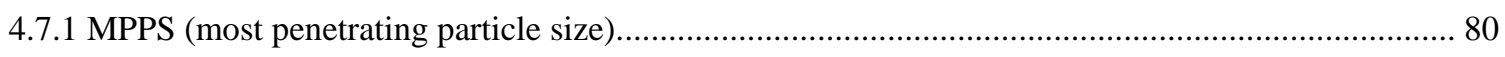

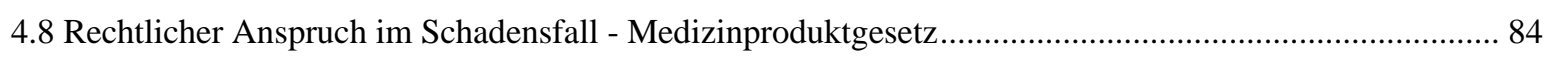

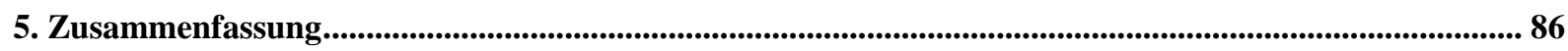

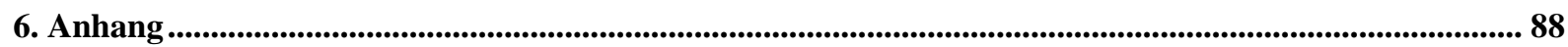

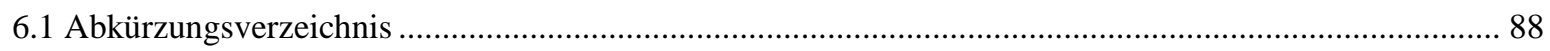

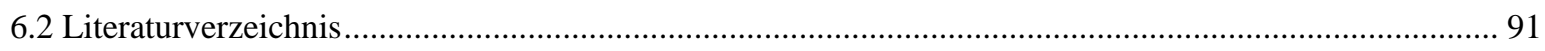

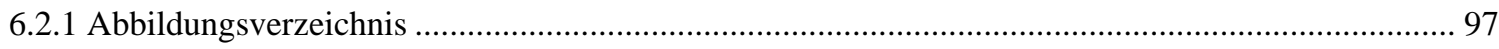

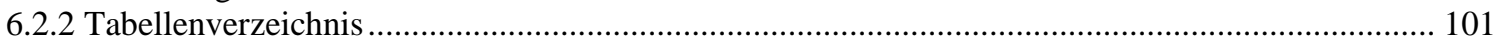

6.3 Rechenschritte und Ergebnisse zu den in der Arbeit ausgeführten Berechnungen................................ 103

6.3.1 Berechnung des Volumenstroms für die in den Kornhäusern gelagerten Testeinheiten und die

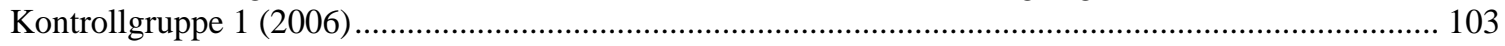
6.3.1.1Volumenstrom durch wetterabhängige Luftdruckänderungen in den Kornhäusern und für die Kontrollgruppe 1 (2006) (Boyle- Mariotte- Gesetz) ................................................................... 103 6.3.1.2 Volumenstrom durch Temperaturänderungen in den Kornhäusern ...................................... 114

(Charles`sches Gesetz)..................................................................................................... 114

6.3.2 Berechnungen des Volumenstroms für die unter realitätsnahen Bedingungen (im medizinischen Bereich) gelagerten Testeinheiten und die Kontrollgruppe 2 (2008).................................................. 122 6.3.2.1 Volumenstrom durch wetterabhängige Luftdruckänderungen in Bad Rothenfelde, Oldenburg und bei der Kontrollgruppe 2 (2008) (Boyle- Mariotte- Gesetz) .................................................... 122

6.3.2.2 Volumenstrom durch Temperaturänderungen am Standort Oldenburg ................................. 135

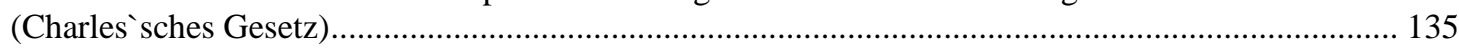




\section{Einleitung}

\subsection{Begriffsdefinition „Steril und Sterilität“}

Der absolute Begriff der „Sterilität“ ist als ein „,von lebensfähigen Mikroorganismen freier Zustand“ (DIN EN 556-1 (2001) S.5) definiert. Die Sterilität eines Medizinproduktes wird durch das Abtöten oder die Inaktivierung vermehrungsfähiger Mikroorganismen durch ein geeignetes Sterilisationsverfahren realisiert.

Hierbei kommt vor allem das Verfahren der Dampfsterilisation, das heißt die Autoklavierung (Bedingungen z.B. $121^{\circ} \mathrm{C}$; 18 Minuten Expositionszeit; gespannter bzw. gesättigter Wasserdampf unter 2,04 bar Absolutdruck) zum Einsatz. Lenkung und Validierung der Sterilisation durch feuchte Hitze ist durch die Norm DIN EN ISO 176651(2006) klar dargestellt, die Leistungsanforderungen an den Sterilisationsprozess an sich sind durch den Normenentwurf DIN EN 285+A2 (2006+2009) vorgegeben. Die Dampfsterilisation stellt das Verfahren der Wahl sowohl im klinischen als auch im industriellen Einsatzbereich dar. Das Prinzip dieser Methode lässt sich durch die Abhängigkeit zwischen Temperatur, Druck und Volumen in einem geschlossenen Gefäß aufzeigen. Als Medium für die Dampferzeugung wird vollentsalztes Wasser verwendet. In einem geschlossenen Gefäß steigt der Siedepunkt des Wassers und damit die aufzubringende Temperatur zur Erreichung des Siedens, somit nimmt auch die Temperatur des Wasserdampfes zu und der Druck des Dampfes erhöht sich. Auf diese Weise kann über den Druck in einem geschlossenen Gefäß (Autoklav) die Temperatur des gespannten Wasserdampfes gesteuert und konstant gehalten werden (DIN EN ISO 17665-1 (2006)). Gespannter Wasserdampf vermag in hohem Maße Energie zu übertragen. Verglichen mit Luft ist die Wärmeleitfähigkeit von gespanntem Wasserdampf ca. 27-fach höher. Die Dampfsterilisation gilt daher im Vergleich zur Heißluftsterilisation als sicher, validierbar und reproduzierbar, muss jedoch geeigneten Prüfverfahren und regelmäßigen Kontrollen im Hinblick auf die Effektivität des Verfahrens unterzogen werden (DIN EN 285+A2 (2006+2009); DIN EN ISO 17665-1 (2006)); Harte und Miller (2004)).

Die kontinuierliche Abnahme der Keimzahl unter Einwirkung des Sterilisationsverfahrens erfolgt gemäß einer Abtötungskinetik erster Ordnung, kann also durch den dekadischen Logarithmus beschrieben werden. In der Praxis wird die Forderung an eine Keimzahlreduktion auf einen Wert von $10^{-6} \mathrm{KbE}(\mathrm{KbE}=$ Kolonie bildende Einheiten) pro Produkt gestellt (Sterilitätssicherheitswert, englisch: sterility assurance level, SAL). Dies bedeutet, dass der „,von lebensfähigen Mikroorganismen freie[...] Zustand“ (DIN EN 556-1 (2001) S.5) erreicht ist, sofern mit einer Wahrscheinlichkeit von $\leq 1: 1.000 .000$ gewährleistet wird, dass nach dem erfolgten Sterilisationsprozess noch ein unsteriles Produkt vorliegen kann (DIN EN 556-1). In diesem Falle „verteilt“ sich ein lebensfähiger Mikroorganismus statistisch gesehen auf 1.000.000 Produkte.

Wichtige Kenngrößen der Sterilisation sind der D-Wert und der z-Wert. Der D-Wert (dezimaler Reduktionswert) gibt die Zeitspanne in Minuten an, die ein Sterilisationsprozess unter bestimmten, vordefinierten Bedingungen (z.B. $121{ }^{\circ} \mathrm{C}, 2,04$ bar Absolutdruck) durchlaufen muss, um eine Keimzahlreduktion einer Ausgangskeimlast um Faktor 10 zu bewirken, das heißt 90\% der initialen Keimlast zu inaktivieren. Der Zahlenwert „z“ gibt die Temperaturdifferenz in K (Kelvin) an, um welche die Behandlungstemperatur erhöht werden muss, damit die Abtötungszeit bei gleicher Keimzahlreduzierung auf 1/10 verkürzt wird (,z“ = zusätzliche Temperatur). Der „,"“- 
Wert hat im wesentlichen wissenschaftliche Bedeutung für die Bewertung der Sterilisationsleistung, der D-Wert wird für die Berechnung der notwendigen Sterilisierzeit herangezogen.

Für die Dampfsterilisation (121 C $\mathrm{C}^{\circ}$ 2,04 bar) beträgt der D-Wert 1,5 Minuten für Endosporen bestimmter bakterieller Sporenbildner (z.B. Bacillus stearothermophilus), die physikalisch und chemisch als besonders resistent gelten und deswegen als Prüforganismen eingesetzt werden. Hierbei spricht man von ReferenzMikroorganismen (DIN EN ISO 17665-1 (2006)). Mit der Inaktivierung der genannten Endosporen werden gleichzeitig sämtliche andere Mikroorganismen kumulativ erfasst.

Demzufolge ergibt sich für diesen Prozess bei Annahme einer hohen, initialen Kontamination von $10^{6}$ Keimen (normativ festgelegt), zunächst eine Zeitspanne von 9 Minuten, die durchlaufen werden muss, um die Kontamination auf $10^{\circ}$ (= 1 Keim) zu minimieren. Daher befände sich unter diesen Bedingungen noch 1 Keim auf dem Produkt. Der Sterilisationsprozess muss aber noch 9 weitere Minuten fortgeführt werden. In diesen weiteren 9 Minuten wird die Keimzahl theoretisch nochmals um Faktor $10^{6}$ abgesenkt.

Somit folgt nach einer insgesamt 18 Minuten andauernden (absoluten) Sterilisierzeit die statistische Wahrscheinlichkeit, dass maximal noch $10^{-6}$ Keime pro Produkt vorliegen, also die anfänglich geforderte Wahrscheinlichkeit, dass maximal ein unsteriles Produkt auf 1.000.000 Produkte auftritt. Demzufolge kann ein Produkt, welches mit diesem Verfahren (Dampfsterilisation bei $121 \mathrm{C}^{\circ}$, 2,04 bar und 18 Minuten Sterilisierzeit) behandelt wurde, nach Abschluss des Prozesses als steril gekennzeichnet werden.

Diese einführenden Beispiele demonstrieren unmittelbar, dass der Prozess der Dampfsterilisation und anderer entsprechender Verfahren ausgiebigen und umfangreichen mathematischen Modellen und Kontrollen unterliegt und somit ein normativ explizit geregeltes, validierbares Verfahren darstellt, welches die Sterilität des Endproduktes gewährleistet.

\subsection{Fokussierung der Problematik „Sterilität“ auf die Barrierewirkung der Verpackung}

Um diesen angemessen hohen Standard des Begriffs „Sterilität“ zu bewahren, der durch die erwähnten Verfahren in einem relativ beachtlichen Aufwand erreicht wird, ist eine angemessene Verpackung und Aufbewahrung des Sterilgutes unabdingbar. Die Hauptanforderung an eine Sterilgutverpackung ist somit die Verhinderung eines erneuten Keimzutrittes nach erfolgter Sterilisation im Sinne einer Rekontamination und die Aufrechterhaltung der Sterilität auf dem Niveau von $10^{-6}$ Keimen pro Produkt. Dies bedeutet also, dass der Zustand der ,,absoluten“ Sterilität praktisch nicht existent ist (die Wahrscheinlichkeit für unsterile Produkte wird durch die Sterilisation asymptotisch geringer). Am Abschluss des Sterilisierprozesses werden die normativen Forderungen der Bezeichnung "steril" erfüllt und danach einzig durch das Verpackungsmaterial in Abhängigkeit von den Umgebungsbedingungen aufrecht erhalten.

Während die Sterilisation als methodisch-normativer Prozess definiert ist und als sicher bezeichnet werden kann, bleibt die Sterilgutverpackung die entscheidende Determinante für eine Rekontamination des Sterilgutes unter Umgebungsbedingungen. Diese Grundanforderung an ein Sterilgutverpackungssystem ist laut dem Deutschen Institut für Normung nach DIN EN ISO 11607-1 klar beschrieben. „Das Ziel eines Verpackungssystems für in der Endverpackung zu sterilisierende Medizinprodukte ist es, die Sterilisation, den physikalischen Schutz und die Aufrechterhaltung der Sterilität bis zum Zeitpunkt der Anwendung sowie die aseptische Bereitstellung zu ermöglichen“ (DIN EN ISO 11607-1 (2009) S.4). 
Daher ist eine effektive Barrierewirkung der Sterilgutverpackung Voraussetzung, um einen erneuten Keimzutritt zu verhindern. Gleichzeitig muss die Sterilgutverpackung es jedoch ermöglichen, das Sterilisiermedium (in der Regel Wasserdampf) auf das zu sterilisierende Produkt wirken zu lassen. Somit stellt die Sterilgutverpackung eine Kompromisslösung dar, wobei einerseits eine Rekontamination durch eine möglichst dichte Verpackung unterbunden werden soll, andererseits jedoch eine gewisse Durchlässigkeit der Sterilgutverpackung unvermeidbar und sogar erwünscht ist, um das Sterilisiermedium effektiv auf das bereits vor der Sterilisation verpackte Sterilgut wirken zu lassen (DIN EN ISO 11607-1 (2009); DIN EN 868-3 (2009); DIN EN 868-7 (2009)). Hierdurch ergibt sich der Vorteil, dass das Sterilgut nach Sterilisierprozess in seiner Endverpackung problemlos dem Sterilisiergerät entnommen werden kann, ohne die Erfordernis besonderer Reinraumbedingungen oder anderer umgebungskeimzahlreduzierender Maßnahmen.

Daher ergibt sich eine zwingend erforderliche Gasdurchlässigkeit der Sterilgutverpackung, die jedoch so gewählt sein muss, dass Keime ausgesperrt bleiben.

„,Die Aufsichtsbehörden erkennen die kritische Natur der Sterilbarrieresysteme an, indem sie sie als Zubehör oder Bestandteil eines Medizinprodukts betrachten“ (DIN EN ISO 11607-2 (2006) S.4 ).

Eine primäre Argumentation könnte verlautbaren, dass eine Wahrung der Sterilität bei Verwendung von Verpackungsmaterialien nach DIN EN ISO 11607-1 sicher gegeben ist. Anzumerken ist jedoch, dass speziell die Papierverpackungen und die Papierseiten der Papier-Folien-Verbundverpackungen zur Dampfsterilisation einen zulässigen Maximalporendurchmesser von $35 \mu \mathrm{m}$ bis $50 \mu \mathrm{m}$ tolerieren (DIN EN 868-3 (2009)). Für die gleichartigen Verpackungen zur Strahlensterilisation werden jedoch $20 \quad \mu \mathrm{m}$ bis $30 \quad \mu \mathrm{m}$ als Maximalporendurchmesser angegeben (DIN EN 868-7 (2009)).

Eine Literaturquelle bezüglich der Begründung dieser Porendurchmesser ist in den entsprechenden DINVorschriften jedoch nicht vorhanden. Es kann davon ausgegangen werden, dass die genannten Größen bezüglich des Porendurchmessers einzig und allein auf rein technischen Erhebungen beruhen, im Sinne der Luftdurchlässigkeit für alternierende Druckänderungen, die während des Sterilisierprozesses erforderlich sind und der Reißfestigkeit.

Es ist also eine gewisse Luftdurchlässigkeit des Papiers erforderlich, um als Verpackungsmaterial für in der Endverpackung zu sterilisierende Produkte eine Durchlässigkeit für gasförmige Sterilisiermittel wie Dampf oder Ethylenoxid zu gewährleisten. „Bei der Prüfung nach ISO 5636-3 darf die Luftdurchlässigkeit des konditionierten Papiers nicht weniger als 3,4 $\mu \mathrm{m} / \mathrm{Pa}$ * s bei einem Luftdruck von 1,47 kPa betragen“ (DIN EN 868-3 (2009) S.7). 
Aus Sicht der Hygiene und Mikrobiologie gestaltet sich die Situation bezüglich des Rekontaminationsrisikos wie folgt.

Freie (lebensfähige) Bakterien weisen typische Durchmesser von 0,5 $\mu \mathrm{m}$ bis $2 \mu \mathrm{m}$ auf. In dieser Größenordnung existieren die Bakterien in freier Form. Häufig liegen sie jedoch verklumpt, in Agglomeraten größeren Durchmessers vor - Größenordnungsbereich liegt zwischen $3 \mu \mathrm{m}$ bis 7,5 $\mu \mathrm{m}$ (Dunkelberg und Schmelz 2008a).

Für native bakterielle Erreger ist das Vorkommen in Form von Aerosolpartikeln (mit einigen Ausnahmen) unüblich, in der Regel treten diese Bakterien in Partikeln oberflächengebunden auf (z.B. auf Hautschuppen bis $>7,5 \mu \mathrm{m}$ ) (Tham und Zuraimi 2005). Demgegenüber können Endo- und Pilzsporen mit Größen von 0,1 $\mu \mathrm{m}$ bis 2 $\mu \mathrm{m}$ häufig hochdispers verteilt in der Luft enthalten sein.

Augustowska und Dutkiewicz konnten in ihrer Veröffentlichung „Variability of airborne microflora in a hospital ward within a period of one year“ im beobachteten Zeitraum nicht nur Verschiebungen in der Zusammensetzung der Luftkeimspezies, sondern auch tägliche und sogar von den Jahreszeiten abhängige Schwankungen der Keimqualität bezüglich Häufigkeit und Keimspezies in der Luft feststellen (Augustowska und Dutkiewicz 2006, S.99). Bei den in diesen Untersuchungen in der Krankenhausluft nachgewiesenen Keimen handelt es sich u. a. vor allem um grampositive Kokken, Endosporen und Pilzsporen. Diese liegen ebenfalls im oben dargestellten Größenordnungsbereich.

Da diese in der Luft enthaltenen Mikroorganismen auftretenden Luftströmen folgen und bei flexiblen Sterilgutverpackungen (hier Papier-Folien-Verbundverpackung) durch Luftdruckänderungen oder starke Temperaturschwankungen ein Volumenein- bzw. Volumenausstrom durchs Verpackungsmaterial erfolgen kann, besteht die Gefahr, dass die Mikroorganismen auf diesem Wege ins Verpackungsinnere gelangen.

Demnach kann die Papierseite der Papier-Folien-Verbundverpackung als eine Art Filtersystem verstanden werden. Bei Betrachtung des physikalischen Prinzips eines solchen Luftfiltersystems muss im Hinblick auf die Wahrung der Sterilität bis zum Anwendungszeitpunkt folgender Sachverhalt kritisch geprüft werden.

Bei einem Luftfilter lässt sich ein bestimmter Partikelgrößenbereich beschreiben, der besonders leicht den Filter passiert (=Filterlücke).

Die Relevanz dieses Partikelgrößenbereiches wird durch seine Berücksichtigung in den DIN Normen 1822 in besonderem Maße bei der „Prüfung des planen Filtermediums“ deutlich. „Anstelle von massenbezogenen Aussagen basiert die neue Technologie auf Partikelzählverfahren im Bereich der Partikelgröße im Abscheidegradminimum (MPPS Most Penetrating Particle Size Bereich : 0,12 $\mu \mathrm{m}$ bis 0,25 $\mu \mathrm{m}$ )“ (DIN EN 1822-3 (2009) S.3). Aufgrund neuer Technologien kann mit besonders hoher Empfindlichkeit der Messinstrumente der integrale Abscheidegrad eines Filtermediums bestimmt werden.

Exemplarisch sei die Darstellung folgender in den DIN Normen (DIN EN 1822-3) abgebildeter Fraktionsabscheidegradkurve. Die MPPS liegt hier bei einer Partikelgröße von 0,16 $\mu \mathrm{m}$, was in diesem Fall einen Abscheidegrad von 99,9975 \% bedeutet. 
Abb. 1:

Modifizierte Darstellung der Fraktionsabscheidegradkurve aus DIN EN 1822-3

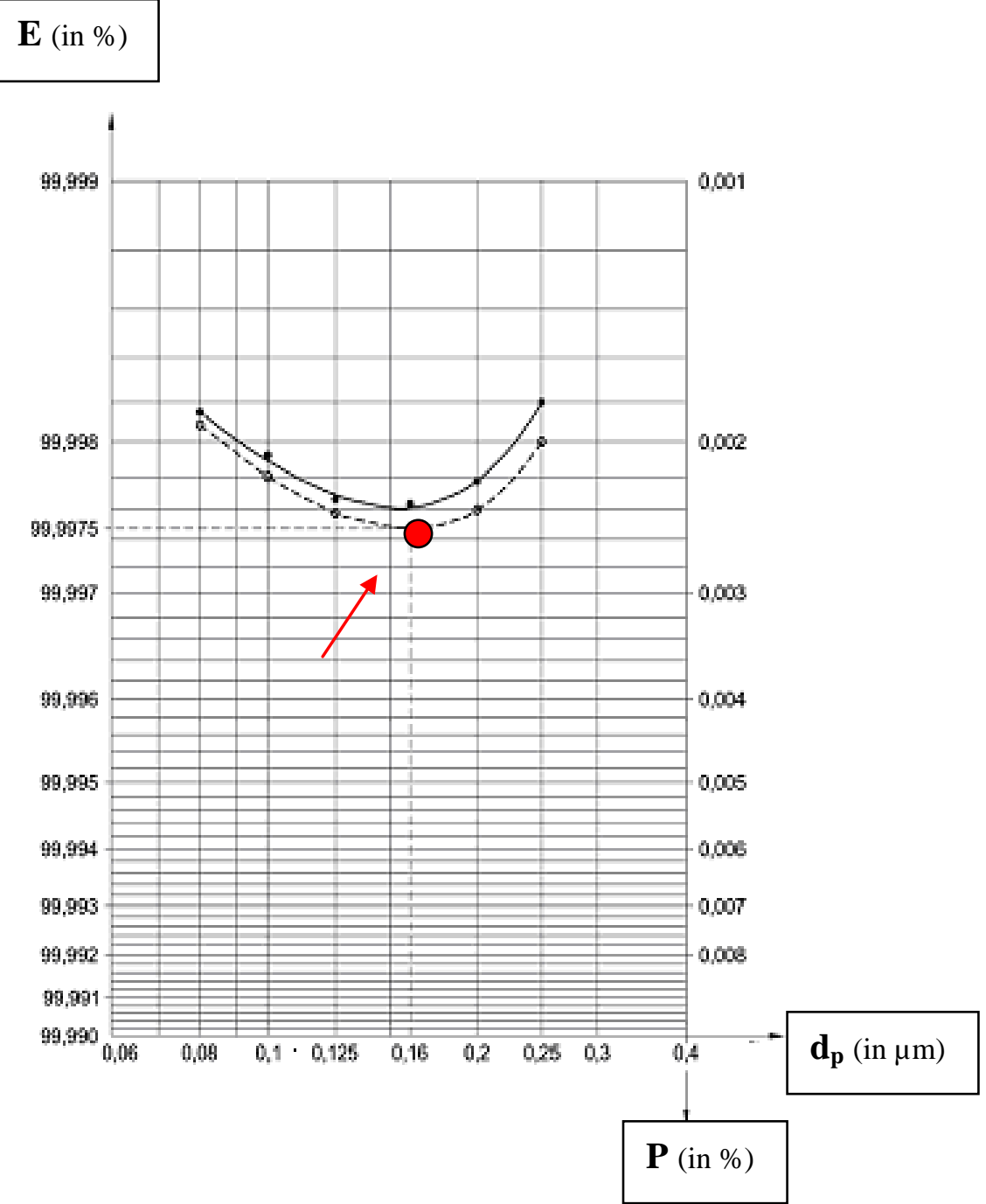

Zu Abb. 1:

Modifizierte Darstellung der Fraktionsabscheidegradkurve aus DIN EN 1822-3

$\mathbf{d}_{\mathbf{p}}=$ mittlerer Partikeldurchmesser

$\mathbf{E}=$ mittlerer Fraktionsabscheidegrad

(der gestrichelte Kurvenverlauf stellt die mittlere Abscheidegradkurve unter Berücksichtigung einer bei Partikelzählungen vorliegenden statistischen Unsicherheit dar)

$\mathbf{P}=$ mittlerer Fraktionsdurchlassgrad

Dieses Diagramm stellt die Filterlücke graphisch dar. Der rote Punkt markiert den mittleren Partikeldurchmesser $\left(d_{p}=0,16 \mu m\right)$ bei dem der mittlere Fraktionsabscheidegrad $(E=99,997 \%)$ am geringsten und der mittlere Fraktionsdurchlassgrad ( $\mathrm{P}=0,003 \%)$ am höchsten ist.

Partikel des mittleren Durchmessers von $d_{p}=0,16 \mu$ merden demnach vom Filtermedium am schlechtesten abgeschieden.

Quelle:

(DIN EN 1822-3 (2009) S.21) 
Auch die Arbeit von Sinclair und Tallentire 2002 (ausführliche Darstellung siehe Abschnitt 4.7.1, Seite 82-84) zeigt für poröses medizinisches Papierverpackungsmaterial einen bestimmten Partikelgrößenbereich auf, welcher bei einwirkenden Druckgradienten besonders schlecht abgeschieden werden kann. Diese schlecht zu filtrierende Partikelgröße liegt hier zwischen $10^{-1} \mu \mathrm{m}$ bis $10^{0} \mu \mathrm{m}$.

Wie bereits erwähnt, messen Endo- aber auch Pilzsporen einen Durchmesser genau in diesem kritischen Größenbereich von ca. 0,5- $2 \mu \mathrm{m}$.

Durch eindringenden Luftstrom kann die Barrierewirkung insbesondere im Hinblick auf Pilzsporen und bakterielle Endosporen vor dem Hintergrund der maximal tolerierbaren Porengröße bei den Sterilgutverpackungsmaterialien von $30 \mu \mathrm{m}$, bzw. $50 \mu \mathrm{m}$, leiden.

Daher wurde im Rahmen dieser Arbeit die These formuliert, dass (unter Zugrundelegen der Durchlässigkeitstoleranzen der DIN EN 868-3 (2009), bzw. 868-7 (2009)) unter anderem bakterielle Endosporen, Mikrokokken und vegetative Pilzsporen unter ungünstigen Bedingungen die Barrierewirkung der Sterilgutverpackung im Sinne einer Papier-Folien-Verbundverpackung durchbrechen können.

Da eine Rekontamination der anfangs sterilen Medizinprodukte somit möglich sein kann, ist es wichtig, für die Barrierewirksamkeit des Verpackungsmaterials gegenüber luftgetragenen Partikeln einen Messwert zu ermitteln. Die Berechnung des LRF (= logarithmischer Reduktionsfaktor) bietet eine Möglichkeit, die Effektivität der Barrierewirksamkeit der genutzten Papier-Folien-Verbundverpackung zu bewerten. Dieser Faktor stellt rechnerisch die Keimreduktionsleistung der Sterilgutverpackung dar, der die logarithmische Verringerung einer initialen Ausgangskeimlast durch die Papier-Folien-Verbundverpackung beziffert.

Ein anderes gleichwertiges Verfahren stellt die Ermittlung des Abscheidegrades (Filtrationswirkung) dar. Beispielsweise entspricht ein LRF = 2 einer Filtrationswirkung von $99 \%$.

\subsection{Lösungsansätze der Rekontaminationsproblematik}

Zur Überprüfung einer gelagerten Charge zwecks Rekontaminationskontrolle könnte man annehmen, dass es hinreichend wäre, exemplarisch 100 oder mehr Einheiten zu öffnen und durch Entnahme eines Abstriches einen Keimbefall zu detektieren. Diese Vorgehensweise ist jedoch mit dem Risiko behaftet, dass bereits bei der Öffnung und unter aseptischen Reinraumbedingungen Keime die Möglichkeit erhalten, an das Sterilgut zu gelangen. Somit würde diese Weise der Testung den Anteil falsch positiver Ergebnisse erhöhen und zugleich die Aussagekraft dieses Systems untergraben.

Ein Prüfungskonzept der Sterilgutverpackungssysteme im Hinblick auf ihre Gesamtheit als Packungseinheit unter Anwendungsbedingungen liegt bislang noch nicht in etablierter Form vor. Da das Verpackungssystem jedoch unabdingbar als eine den Sterilisationsprozess und die Lagerung durchlaufende Einheit zu betrachten ist, existiert bislang keine geeignete, allgemein anerkannte Prüfmethodik dieses komplexen Sterilgutverpackungssystems.

Dem Anwender der Medizinprodukte wird ein Verpackungsmaterial zur Verfügung gestellt, welches die entsprechenden Leistungsanforderungen nach DIN EN 868 erfüllen muss. Für die Anwendung dieses geeigneten Verpackungsmaterials, also die korrekte Handhabung, Lagerung und die Aufrechterhaltung der Sterilität des Medizinproduktes im Praxisalltag bis zum Zeitpunkt der Anwendung, ist der Anwender selbst verantwortlich. Hierzu werden ihm Normen bereitgestellt, um diesen Anwendungsprozess reproduzierbar und validierbar zu 
gestalten. „Die Anwendung dieser Norm zusammen mit den Normen DIN EN ISO 11607-1 (2009), DIN EN ISO 11607-2 (2006) und DIN 58953-8 (2010) stellt sicher, dass Medizinprodukte, wie z. B. Instrumente und Sets in Einrichtungen für die Gesundheitsfürsorge und deren Dienstleistungsbetrieben in einem Sterilbarrieresystem einwandfrei verpackt und gehandhabt werden können“ (DIN 58953-7 (2010) S. 4).

Gleichzeitig mangelt es jedoch an einem geeigneten Prüfverfahren, anhand dessen der Anwender die Effektivität seiner Bemühungen im Hinblick auf die Sterilhaltung des verpackten Medizinproduktes nachweisen kann.

Da eine Wiederverkeimung möglich ist, befindet sich das Sterilgut in Abhängigkeit von den Lagerungsbedingungen unmittelbar vor der Anwendung in einem unkontrollierten mikrobiologischen Zustand.

Dass die kritische Betrachtung der Barrierewirkung einer Sterilgutverpackung kein medizintheoretisches Konstrukt darstellt, zeigt der Normenentwurf der International Standardizing Organisation: „Es gibt kein allgemein anerkanntes Verfahren für den Nachweis der mikrobiellen Barriereeigenschaften. [...] Sobald validierte mikrobielle Belastungsverfahren für Materialien und Sterilbarrieresysteme verfügbar sind, wird deren Aufnahme in zukünftige Ausgaben von diesem Teil von ISO 11607 erwogen “ (DIN EN ISO 11607-1 (2009) S.14).

Hier zeichnet sich also eine normative Forderung (!) für die Entwicklung geeigneter mikrobiologischer Methoden zum Nachweis der Barrierewirkung ab.

\subsection{Anforderungen an ein geeignetes Prüfsystem}

Im Rahmen der hier vorgelegten Untersuchung werden folgende Anforderungen an ein geeignetes Prüfsystem gestellt:

1. Das Testverfahren sollte die individuelle Sterilgutverpackung als gesamte Einheit prüfen.

2. Die Testung der Sterilgutverpackungen muss unter den konkreten Umgebungs- und Lagerungsbedingungen praxisnah durchgeführt werden.

3. Die Prüfung der Barrierefunktion der Sterilgutverpackung sollte selbst kein Rekontaminationsrisiko beinhalten.

Ähnliche Prüfkonzepte, welche die Kontrolle der Barrierewirkung der Sterilgutverpackung als Funktionseinheit unter Anwendungsbedingungen ermöglichen und damit die drei aufgeführten Forderungen erfüllen, wurden inzwischen theoretisch und experimentell untersucht (Dunkelberg und Wedekind 2002; Dunkelberg und Wedekind 2004; Dunkelberg und Fleitmann- Glende 2006) und werden im folgenden Abschnitt (Abschnitt 1.5, Seite 13) kurz beschrieben. 


\subsection{Darstellung bereits getesteter Verpackungssysteme}

Seit dem Jahr 2000 wurde eine Reihe von Testungen verschiedener Verpackungssysteme mit dem Expositionskammerverfahren unter Anwendung unterschiedlicher Determinanten (Druckwechsel: Häufigkeit und Kinetik der Wirkung des Druckes, Luftkeimzahl) im Institut für Hygiene und Umweltmedizin der Universitätsmedizin Göttingen durchgeführt. Diese Versuche führten zu dem Ergebnis, dass die Barriere unbeschädigter Sterilgutverpackungssysteme durch aerogene Keime durchbrochen werden kann. Die Testungen erfolgten zunächst an Papier-, Doppelpapier- und Tuch- Papierverpackungen (Dunkelberg und Wedekind 2002; Dunkelberg und Wedekind 2004). Anschließende Versuchsreihen beschäftigten sich mit der Barrierewirkung von Sterilgutcontainern mittels Expositionskammer (Dunkelberg und Fleitmann- Glende 2006). Verschiedene Verpackungssysteme wurden hierbei alternierenden Druckwechseln in einer Expositionskammer definierten Zeiträumen ausgesetzt.

Wenngleich durch die Expositionskammerversuche viele wichtige Ergebnisse bezüglich der Barrierewirksamkeit ermittelt wurden, konnten diese Laborergebnisse nicht in allen Aspekten auf die Praxis übertragen werden, was Lagerung und Handhabung des Verpackungsmaterials betrifft.

Genau dieser Sachverhalt der nicht unmittelbar auf die Praxis übertragbaren Ergebnisse von Untersuchungen mit der Expositionskammer wird durch die Untersuchungen der vorliegenden Promotionsarbeit im Feldversuch ergänzt.

Bereits im Jahr 2006 wurde im Institut für Hygiene und Umweltmedizin ein Testkit entworfen, welches nach dem Sterilisationsprozess in drei verschiedenen Kornhäusern eingelagert wurde.

Die Testeinheit wurde entwickelt, um als „Double“ verpackter Medizinprodukte Anwendung zu finden. Sie besteht aus einem Aktivierungsteil und einem mikrobiologischen Anteil (Nährkartonscheibe). Nähere Erläuterungen zur Funktionsweise und zum Aufbau dieser Bestandteile sind in Abschnitt 2 (Seite 20 - 25) zu finden.

Die Verpackungssysteme wurden in den Kornhäusern den dort herrschenden Umgebungsbedingungen ausgesetzt (Temperatur, atmosphärische Druckschwankungen, hohe Umgebungskeimlast der Luft). Nach Lagerung und Transport konnte ein Nährkarton im Inneren der Verpackung, ohne die Verpackungseinheit zu öffnen, aktiviert werden. Hierbei zeigte sich eine Verkeimung der im Sterilgutverpackungssystem integrierten Testeinheit von bis zu 84,2 \%. Dieses Ergebnis unterstreicht die Notwendigkeit, dieses Testsystem auch unter praxisbezogenen Bedingungen einzusetzen.

In der hier vorliegenden Arbeit werden die eigenen Untersuchungen diesen früher bereits im Jahr 2006 durchgeführten, jedoch noch nicht ausgewerteten Untersuchungen vergleichsweise gegenübergestellt (Darstellung der Rohdaten aus den Kornhäusern siehe Abschnitt 1.7, Seite 16 - 19 ). 


\subsection{Zielsetzung}

Ziel dieser Untersuchung war somit die Weiterentwicklung einer Methode bzw. eines Instrumentes zur Überprüfung der Barrierewirksamkeit einer Sterilgutverpackungseinheit nach Lagerung und Transport.

Hierzu wurde eine Testeinheit als „Double“ der zu testenden Sterilgutverpackungssysteme angefertigt und bezüglich der täglichen Handhabung, der Lagerung und des Transports gleich behandelt (Aufbau der entwickelten Testeinheit ist Abschnitt 2 auf Seite 20 - 25 zu entnehmen).

Für die entwickelte Testeinheit unter praxisüblichen Bedingungen ergeben sich wie bereits ausgeführt folgende auf die Verpackung wirkende Einflussfaktoren:

- $\quad$ atmosphärische Druckschwankungen

- Temperaturschwankungen

- $\quad$ aerogene Umgebungskeimlast unter Lagerungsbedingungen.

Um eine Variation dieser auf die Verpackung wirkenden Einflussfaktoren zu erhalten, sollte die Lagerung der Testeinheiten an verschiedenen Standorten vorgenommen werden. Das genaue Konzept der Lagerung wird in Abschnitt 2.6 (Seite 28 - 30) ausführlich dargestellt, an dieser Stelle soll vorab lediglich ein kurzer Überblick gegeben werden.

Die in Bad Rothenfelde (im OP-Bereich dieser Herzklinik) gelagerten Testeinheiten waren durch klimatisierte Räumlichkeiten nur geringen Temperaturschwankungen bei üblichen witterungsbedingten atmosphärischen Druckwechseln ausgesetzt.

Eine weitere Charge wurde in einem Sterilgutlager in Oldenburg deponiert, auch hier wirkten die wetterabhängigen atmosphärischen Druckschwankungen auf die Verpackungseinheiten, die Temperaturen entsprachen jedoch den vorherrschenden Umgebungstemperaturen der Außenluft.

Anhand der zu ermittelnden Druck- und Temperaturschwankungen sollten unter Anwendung des BoyleMariotte- Gesetzes (Druckänderungen, siehe Abschnitt 2.8.1, Seite 33) und des Charles'schen Gesetzes (Temperaturschwankungen, siehe Abschnitt 2.8.2, Seite 34) die Volumenänderung der Luft im Verpackungsinneren und somit auch die einströmenden Luftmengen in die Testeinheit berechnet werden.

Aufgrund der ermittelten Umgebungskeimzahlen an den entsprechenden Lagerungsstandorten und der berechneten einströmenden Luftvolumina ins Verpackungsinnere wurde die mikrobielle Beanspruchung der Verpackung während Lagerung und Transport abgeschätzt.

Durch diese Abschätzung der mikrobiellen Beanspruchung des Verpackungssystems unter gegebenen Lagerungs- bzw. Praxisbedingungen sollte in Verbindung mit den Testresultaten eine Aussage über die Effektivität der Barrierewirkung bzw. Filterwirkung der Papierseite getroffen werden.

Auf der Grundlage der bereits 2006 und somit vor Beginn dieser Arbeit durchgeführten Vorversuche wurden die damals ermittelten Rohdaten (siehe Abschnitt 1.7, Seite 16 - 19) im Rahmen der hier vorgelegten Arbeit zur Berechnung der Filtrationswirkung und des logarithmischen Reduktionsfaktors des Verpackungsmaterials weiter ausgewertet.

Unter Berücksichtigung der Filtrationswirkung und der mikrobiellen Beanspruchung im klinischen Umfeld sollte weiter eine Abschätzung der Rekontaminationswahrscheinlichkeit für die gewählten Lagerungsbedingungen erfolgen und mit den tatsächlich ermittelten Versuchsergebnissen verglichen werden. 


\section{Konzeptioneller Einsatz der Testeinheit als Verfahren zur Überprüfung der Aufrechterhaltung der Sterilität zum Zeitpunkt der vorgesehenen Anwendung des Sterilproduktes im Praxisalltag}

Wenn die verpackte Testeinheit also exakt wie weitere den Sterilisierprozess durchlaufende Sterilgüter behandelt wird, kann sie gleichsam als „Double“ der standardmäßigen Sterilgüter gewertet und beurteilt werden.

Somit bestand in diesem Feldversuch die Möglichkeit, die Testeinheit als eine Art „Prüfkörper“ für das Sterilprodukt hinzuzufügen und während des gesamten Zeitintervalls von Sterilisation, Lagerung und Distribution bei der Sterilgutcharge zu belassen. Auf diese Weise wurden die Testeinheiten denselben Bedingungen (zum Beispiel Umgebungskeimlast, Temperatur- oder atmosphärischen Druckschwankungen) ausgesetzt, wie es bei üblicherweise mitgeführten Medizinprodukten der Fall ist.

Bei Anwendung dieser Methode im Praxisalltag kann bei absehbarer Verwendung der betreffenden Medizinprodukte 48 Stunden zuvor die mitgeführte Testeinheit ausgewertet und die Medizinprodukte nach erhaltenem Ergebnis, bei vorliegender Sterilität, zur Benutzung freigegeben werden. Folglich bedeutet dies, dass ein negativer Befund bei der Auswertung der Testeinheiten im Hinblick auf eingedrungene Mikroorganismen auch auf die übrigen Testeinheiten übertragen werden kann. Hierbei ist jedoch zu berücksichtigen, dass die Auswertung einer mitgeführten Testeinheit als eine Stichprobe zu verstehen und zu deuten ist. Je größer die Anzahl der geprüften Testeinheiten ist, desto höher wird die Aussagekraft dieser Stichprobe.

Der Zustand der Testeinheit spiegelt mittels dieser Methode dementsprechend genau den Zustand der mitgeführten Medizinprodukte wider, indem durch die Testeinheit die Beschaffenheit und Ausführungsform der „Originalverpackungen“ im Vergleich mit dem Sterilgut exakt nachgeahmt wird und gleichzeitig denselben Umgebungsdeterminanten ausgesetzt wird.

Infolgedessen bieten die Testeinheiten die Möglichkeit, die Aufrechterhaltung der Sterilität analog zum sterilisierten Medizinprodukt unmittelbar vor dessen geplanter Benutzung individuell und chargenabhängig zu ermitteln. Darüber hinaus kann klinikintern bei definierter Sterilguthandhabung und -logistik das Procedere bezüglich der Angaben zur maximalen Lagerzeit überprüft und optimiert werden. 


\section{7 „Worst- Case- Szenario“ für die zu prüfende Testeinheit: Stand der Ergebnisse zu Beginn der hier vorgelegten Studie}

Bereits im Jahr 2006 wurde im Institut für Hygiene und Umweltmedizin eine Testeinheit eingesetzt, welche nach Durchlaufen des Sterilisationsprozesses in drei verschiedenen Kornhäusern eingelagert wurde und sich zur Prüfung von Papier-Folien-Verbundverpackungen eignet. Im Aufbau entsprach die Testeinheit dem im Rahmen dieser Arbeit verwendeten Testsystem.

Die Verpackungssysteme wurden den dort herrschenden Umgebungsbedingungen ausgesetzt (Temperatur, atmosphärische Druckschwankungen, hohe Umgebungskeimlast der Luft).

Im Hinblick auf die üblicherweise im Klinikalltag (Lagerung in klimatisierten, geschlossenen Räumlichkeiten ohne das Vorkommen einer hohen Umgebungskeimlast der Luft) Anwendung findenden Papier-FolienVerbundverpackungen stellt der Expositionsort in den Kornhäusern somit eine Art „Worst- Case- Szenario“ dar.

Die hierzu exponierten Testeinheiten wurden in folgender Weise an den verschiedenen Standorten deponiert:

Die Aufzüge in den drei Kornhäusern (Dransfeld, Obernjesa und Scharzfeld) wurden mit jeweils 95 Testeinheiten bestückt, wodurch in Abhängigkeit von der Nutzungsfrequenz der Aufzüge alternierende atmosphärische Druckwechsel zu entsprechenden einströmenden Luftvolumina in die Testeinheiten führten.

Die entsprechenden Luftdruckunterschiede lagen bei 2,5 hPa (Dransfeld und Scharzfeld) bzw. 3 hPa (Obernjesa) für eine Auf- und Abwärtsfahrt.

Zur Dokumentation der Häufigkeit der Nutzung der Aufzüge wurde eine Liste im Aufzug installiert, auf welcher das Personal jede durchgeführte Auf- und Abwärtsfahrt verzeichnete.

Somit waren die Testeinheiten definierten, durch die Aufzugsfahrten bedingten Druckwechseln sowie weiter den witterungsbedingten Luftdruckschwankungen ausgesetzt.

Eine vierte Kohorte mit 96 Testeinheiten wurde im Kornhaus Obernjesa als eine Art „stationäre Lagerung“ außerhalb des Aufzugbetriebes an einer fixen Position platziert. Somit wirkten hier lediglich die wetterabhängigen Luftdruckänderungen auf diese vierte Kohorte der „stationären Lagerung“.

Die in den Kornhäusern gelagerten Testeinheiten waren zudem nahezu den vor Ort herrschenden Außentemperaturen ausgesetzt.

Im Gegensatz zu den Lagerungsbedingungen für Papier-Folien-Verbundverpackungen in Kliniken (teilweise unter Reinraumbedingungen) ist die mikrobielle Beanspruchung des Verpackungssystems im Hinblick auf die hohe Umgebungskeimlast der Luft in den Kornhäusern anders zu bewerten.

Eine Luftkeimzahlbestimmung der Umgebungsluft wurde hierzu im Rahmen der Vorversuche 2006 in Obernjesa durchgeführt. Im Hinblick auf die Schimmelpilze wurde eine Konzentration von 7,8*10 $\mathrm{KbE} / \mathrm{m}^{3}$ festgestellt (Pilzgattungen: Aspergillus und Rhizopus). Für Bakterien ergab sich eine Keimzahl von 0,503*10 $10^{6} \mathrm{KbE} / \mathrm{m}^{3}$ (Bakterien: grampositive Stäbchen und grampositive Kokken). Insgesamt ergab sich somit eine Keimzahl von $8,3 * 10^{6} \mathrm{KbE} / \mathrm{m}^{3}$ 
Der auf diese Weise für die Kornhäuser ermittelte Wert der aerogenen Umgebungskeimlast von $8,3 * 10^{6} \mathrm{KbE} / \mathrm{m}^{3}$ erscheint auf den ersten Blick recht hoch. Hier sei jedoch auf eine Studie von Swan und Crook 1998 verwiesen, die ebenfalls entsprechend hohe Umgebungskeimzahlen ermittelt haben. Im Rahmen der Untersuchung des Einflusses von Getreidestaub auf die Gesundheit der in der Getreideverarbeitung wirkenden Arbeiter wurden folgende Werte für die mikrobiologische Belastung der staubigen Luft erhoben: „Concentrations of airborne bacteria ranged from $1,2 * 10^{5}$ to $1,3 * 10^{7} \mathrm{cfu} / \mathrm{m}^{3}$.” „Concentrations of airborne fungual spores ranged from $1,8^{*} 10^{3}$ to $1,3^{*} 10^{7} \mathrm{cfu} / \mathrm{m}^{3}$ ” (Swan und Crook 1998, S.10-11).

Entsprechend hohe Werte der mikrobiologischen Belastung der Umgebungsluft wurden auch von KrysińskaTraczyk et al. 2005 im Rahmen einer Studie über den Einfluss der Verarbeitung von Getreideprodukten auf die Arbeiter ermittelt. „.... [V]ery large microbial concentrations of the order $10^{6}-10^{7} \mathrm{cfu} / \mathrm{m}^{3}$ were recorded” (Krysińska- Traczyk et al. 2005, S.273).

Als Kontrollgruppe (Kontrollgruppe 1) wurden weitere 100 Testeinheiten angefertigt, die nach Durchlaufen des Sterilisationsprozesses zur Lagerung im Institut für Hygiene und Umweltmedizin in Göttingen verblieben.

Auf diese Testeinheiten wirkten lediglich die wetterabhängigen atmosphärischen Druckschwankungen und aufgrund klimatisierter Räumlichkeiten eine tägliche geschätzte Temperaturdifferenz von $1^{\circ} \mathrm{C}$.

Zur Bestimmung der Luftkeimzahl wurden hier Daten von innenraumlufttechnischen Erhebungen in Göttingen herangezogen. Als Bezugswert wurde hierbei immer auch die Luftkeimzahlbestimmung der äußeren Umgebungsluft ermittelt. Aus einer Sammlung verschiedener Messdaten ergab sich hierbei die approximative Luftkeimzahl von $350 \mathrm{KbE} / \mathrm{m}^{3}$ in Göttingen.

\section{Prüfung der Autoklavierbarkeit der in der Testeinheit integrierten Nährkartonscheibe}

Des Weiteren wurde die Autoklavierbarkeit der angewandten Nährkartonböden ohne Verlust der Kultureigenschaften nachgewiesen.

Durch die feuchte Hitze des Sterilisationsprozesses war eine Beeinflussung der Nährstoffe und somit der Anzüchtung sedimentierter Keime nicht auszuschließen.

Daher empfahl es sich in diesem Fall aus der auf Seite 27 aufgeführten Tabelle 2 verschiedene Keimarten exemplarisch auszuwählen und mit folgenden Testkeimen zwei Versuchsreihen parallel ablaufen zu lassen:

- Staphylococcus aureus

- Bacillus subtilis

- $\quad$ Pseudomonas aeroginosa

- $\quad$ Pilze.

Hierzu wurden die in den Testeinheiten verwandten Nährkartonböden mit aufgenähtem Membranfilter einem konventionellen CASO- Agar gegenübergestellt. Es erfolgte eine Überimpfung der jeweiligen Nährmedien und anschließend der Vergleich beider Versuchsreihen. Diese in Vorversuchen (2006) im Institut für Hygiene und Umweltmedizin in Göttingen durchgeführten Testungen, führten zu dem Ergebnis einer guten Eignung der Nährkartonböden für die Anwendung in der entwickelten Testeinheit. Die Nährkartonböden konnten somit problemlos für die Produktion der Testeinheiten für Bad Rothenfelde, Oldenburg und die Kontrollgruppe 2 zum Einsatz kommen. 


\section{Zusammenfassung der im Vorversuch 2006 ermittelten Ergebnisse}

Nach Transport und Lagerung in den Kornhäusern konnte der in der Papier-Folien-Verbundverpackung integrierte Nährkarton, ohne die Verpackungseinheit zu öffnen, aktiviert werden.

Das Nährmedium wurde befeuchtet und kultiviert, um einen Nachweis möglicher eingedrungener Mikroorganismen zu erbringen (siehe auch Methodik unter Abschnitt 2).

Die hieraus resultierenden Rohdaten, welche bereits teilweise auf Vorträgen der Fachwelt vorgestellt , jedoch noch nicht weiter ausgewertet wurden, werden im Folgenden kurz zusammengetragen:

Tabelle 1:

Im Vorversuch 2006 ermittelte Rohdaten

\begin{tabular}{|c|c|c|c|c|}
\hline $\begin{array}{c}\text { Testgruppe } \\
\text { Lagerungsort }\end{array}$ & $\begin{array}{c}\text { Lagerungsdauer } \\
\text { (in Monaten) }\end{array}$ & $\begin{array}{c}\text { (n) } \\
\text { Anzahl } \\
\text { Testeinheiten }\end{array}$ & $\begin{array}{c}\left.\text { (n } \mathbf{n}_{\text {unsteril }}\right) \\
\text { Anzahl } \\
\text { unsteriler } \\
\text { Testeinheiten }\end{array}$ & $\begin{array}{c}\text { Prozentualer Anteil (\%) } \\
\text { unsteriler Systeme an } \\
\text { der Gesamtsystemzahl }\end{array}$ \\
\hline Dransfeld (Aufzugsbetrieb) & 4 & 95 & 74 & 77,9 \\
\hline Obernjesa (Aufzugsbetrieb) & $31 / 2$ & 95 & 80 & 84,2 \\
\hline Obernjesa(,stationäre Lagerung“) & $31 / 2$ & 96 & 53 & 55,2 \\
\hline Scharzfeld (Aufzugsbetrieb) & $31 / 2$ & 95 & 66 & 69,5 \\
\hline Kontrollgruppe 1 & 6 & 100 & 0 & 0 \\
\hline
\end{tabular}

Somit erwies sich der überwiegende Anteil der deponierten Testeinheiten nach Lagerung, Transport und Aktivierung des mikrobiologischen Anteils des Testsystems als unsteril.

Die Quantifizierung der in die Testeinheiten eingedrungenen Mikroorganismen erbrachte im Falle der Kornhäuser folgendes Ergebnis. Sie erfolgte für den Standort Obernjesa sowohl bei den in den Aufzügen gelagerten Testeinheiten als auch bei den stationär gelagerten Testeinheiten ohne Einfluss durch den Aufzugsbetrieb.

\section{Obernjesa mit Aufzugsbetrieb}

Nach Abschluss der Lagerungsdauer, Aktivierung und Auswertung des mikrobiologischen Anteils der Testeinheiten wurde die Häufigkeitsverteilung von Pilzkolonien pro Testeinheit bestimmt. Hierbei wiesen 15 Testeinheiten keinerlei Pilzbefall auf, 11 Einheiten wurden wegen des Überwachsens des Nährkartons aus der Wertung genommen (keine Zählung möglich). Bei dem größten Teil der Testeinheiten (30 Stück) wurde eine Pilzkolonie gezählt. Weitere 18 Testsysteme wiesen 2 Kolonien auf und 3 Pilzkolonien waren auf 12 Nährkartonscheiben zu finden. Bei weiteren 10 Testeinheiten waren 4 bzw. 5 Pilzkolonien zu erkennen.

Obernjesa ohne Aufzugsbetrieb - „stationäre Lagerung“

Hierbei wiesen 43 Testeinheiten keinerlei Pilzbefall auf, 5 Einheiten wurden wegen des Überwachsens des Nährkartons aus der Wertung genommen (keine Zählung möglich). 
Lediglich bei 19 Testeinheiten war eine Pilzkolonie festzustellen und 2 Pilzkolonien wurden auf den Nährböden von 21 Testeinheiten ausgemacht. Die Anzahl von 3 - 6 Pilzkolonien wurde bei wenigen Testeinheiten vereinzelt gezählt.

Im Gegensatz zu den im Aufzug deponierten Testeinheiten ist bei den Testeinheiten der „stationären Lagerung“ ein wesentlich geringerer Pilzbefall der Testsysteme zu erkennen.

Diese 2006 durchgeführten Untersuchungen wurden bereits teilweise auf Vorträgen der Fachwelt vorgestellt. („Entwicklung eines Testkits zur Überwachung der Barriereeigenschaften von Sterilgutverpackungssystemen im Krankenhausroutinebetrieb - Erste Ergebnisse“ (Dunkelberg und Schmelz 2007; Vortrag im Rahmen des 19. DOSCH- Symposiums, Millstatt/ Kärnten 21. - 23. Mai 2007)).

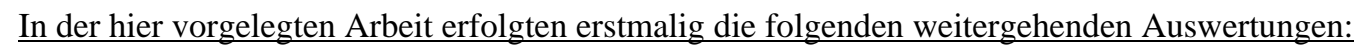

- Ermittlung des einströmenden Luftvolumens in die Testeinheiten als Folge atmosphärischer Druckschwankungen (Boyle- Mariotte`sche Gesetz)

- Ermittlung des einströmenden Luftvolumens in die Testeinheit als Folge der Temperaturschwankungen (Charles'sches Gesetz)

Somit war die Möglichkeit gegeben, die Häufigkeit kontaminierter Testeinheiten in Abhängigkeit von der mikrobiologischen Beanspruchung, d.h. dem Produkt aus einströmendem Luftvolumen und der Luftkeimzahl, auszuwerten.

- $\quad$ Ermittlung des logarithmischen Reduktionsfaktors (LRF) und der Filtereffektivität

Der Rückgriff auf die 2006 in den Vorversuchen erhobenen Rohdaten und deren erstmalige Auswertung im Rahmen dieser Arbeit waren erforderlich, um abschließend zu einer Einschätzung der mikrobiellen Beanspruchung und Filtrationswirksamkeit der Papier-Folien-Verbundverpackungen bei den entsprechenden Expositionsszenarien zu gelangen und um die Ergebnisse zur Häufigkeit der Rekontamination exponierter Testeinheiten in Relation zu den jeweiligen Expositionsbedingungen und zu der ermittelten Filterwirksamkeit der Verpackung darzustellen. 


\section{Material und methodisches Konzept}

\subsection{Materialien zur Herstellung}

\section{Materialien für die Testeinheiten}

- $\quad$ Reagenzgläser 160 * 16mm Fiolax-Glas, Fa. Schott-Laborglas Mainz

- $\quad$ Silikonschlauch, Innendurchmesser $16 \mathrm{~mm}$

- $\quad$ Silikonstopfen $14 * 19 \mathrm{~mm}$

- $\quad$ Petrischale, Glas, Durchmesser 60mm, Höhe 15mm, VWR Darmstadt (Nr.391F2710)

- Standard-Nährkartonscheiben Durchmesser 50mm, Fa. Möller \& Schmelz Göttingen (Nr.1190-11)

- Membranfilter aus Cellulosenitrat 0,65 Mm Porendurchmesser, Fa. Sartorius Göttingen (Nr. 13005-50ACN)

- Glaswolle

- Individuelle Sterilgutverpackungen - Papier-Folien-Verbundverpackung, Fa. Stericlean

- Nähmaschine und Nähgarn

- $\quad$ Butangas- Gasbrenner

- Inkubator (Standardgerät mit $36^{\circ} \mathrm{C}$ Konstanttemperatur)

- Autoklav

- $\quad$ Brutschrank

- $\quad$ Pipette

- $\quad \mathrm{NaCl}$ ( 0,9\%ige Natrium- Chlorid- Lösung)

- Wattestäbchen (für Abstriche und zum Übertragen der Keime auf den CASO- Agar)

- $\quad$ Aqua dest.

\section{Materialien zur Qualitätsprüfung der Testeinheiten}

- $\quad$ Siegelnahttestlösung (Rhodamin B- Lösung)

o $\quad 94,7 \%$ Aqua dest.

o $5 \%$ Isopropanol

o $\quad 0,15 \%$ Tensid (TWEEN 80)

o $\quad 0,15 \%$ Rhodamin B (Farbstoff)

- $\quad$ Testkeime
o Staphylococcus aureus
o Bacillus subtilis
o Pseudomonas aeruginosa
o Pilze

\section{Materialien zum Anzüchten möglicher sedimentierter Keime}

- CASO- Agar (Casein- Sojalecithin- Agar) 


\subsection{Prinzip der Testeinheit}

Im Zentrum dieser Untersuchung steht eine im Institut für Hygiene und Umweltmedizin entwickelte Testeinheit, die in der zu testenden Sterilgutverpackung (Papier-Folien-Verbundverpackung) integriert ist und eine Einheit mit dieser bildet. Diese Testeinheit besteht aus zwei Komponenten, einem mikrobiologischen Anteil (Nährkartonscheibe) zum Nachweis eingedrungener Keime und einem Aktivierungsteil, welcher die Bewässerung des mikrobiologischen Anteils ohne Öffnen der Papier-Folien-Verbundverpackung möglich macht. Die Testeinheit wurde anstelle des eigentlichen Medizinproduktes auf gleiche Weise verpackt. Es bestand nun die Möglichkeit, dass die Testeinheit parallel zu den anderen verpackten Medizinprodukten als eine Art „Double“ alle Arbeitsschritte durchläuft, die das übliche Sterilgut bis zur Anwendung erfährt. Dieses „Double“ wurde entsprechend üblichen Medizinprodukten des Praxisalltags sterilisiert, transportiert und gelagert. Die entwickelte Testeinheit machte somit den Alterungsprozess und Expositionsprozess der Verpackung durch, wie auch dies im medizinischen Alltag der Fall ist.

Nach abgeschlossener Lagerung konnte die in der Papier-Folien-Verbundverpackung enthaltene Testeinheit aktiviert werden und eine potenzielle Rekontamination des sterilisierten Inhalts nach Lagerung und Transport nachweisen, ohne das Verpackungssystem öffnen zu müssen. Dies war durch einen in die Testeinheit integrierten mikrobiologischen Anteil möglich, welcher nach Aktivierung die Nachweisbarkeit in das Verpackungssystem eingedrungener Keime ermöglichte.

\subsection{Aufbau der Testeinheit}

\subsubsection{Mikrobiologischer Teil der Testeinheit}

Dieser Teil der Testeinheit besitzt als Grundstruktur und gleichzeitig als Haltevorrichtung für die Nährkartonscheibe eine Petrischale. Die Petrischale wurde in ihrer Größe entsprechend der in dieses Behältnis spannungsfrei, jedoch mit gewisser Klemmpassung einzulagernden Nährkartonscheibe ausgewählt. Die Nährkartonscheiben konnten so in gewisser Weise auf dem Boden der Petrischale fixiert werden. Zusätzlich erfolgte das Aufnähen eines Membranfilters auf den Nährkarton, um das Wachstum von Einzelkolonien zu ermöglichen. Damit stand die Nährlösung vollständig den auf dem Membranfilter wachsenden Kulturen zur Verfügung.

Des Weiteren war die Positionierung der Petrischale in der Papier-Folien-Verbundverpackung von entscheidender Bedeutung. Der Papierseite der Verpackung sollte hier die offene Seite der Petrischale zugewandt sein, um eine Sedimentation der die Barrierefunktion durchbrechenden Mikroorganismen zu ermöglichen. 
Abb.2:

Querschnitt durch eine Testeinheit mit darin befindlicher, präparierter Petrischale

Die Barrierefunktion durchbrechende Mikroorganismen

Papierseite

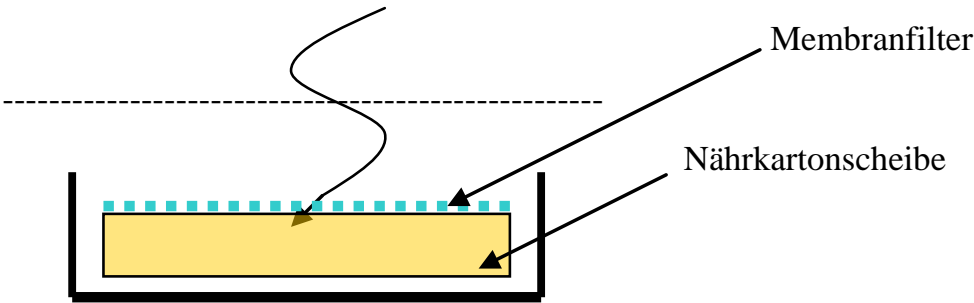

Folienseite

zu Abb. 2:

Querschnitt durch eine Testeinheit mit darin befindlicher, präparierter Petrischale

Keime haben durch die für den Sterilisationsprozess notwendigen Poren in der Papierseite die Möglichkeit, in die Verpackung einzudringen und auf den Nährkarton zu sedimentieren

modifiziert nach Dunkelberg und Schmelz (2008 b)

Testkit zur routinemäßigen Überwachung der Barriereeigenschaften von Sterilgutverpackungssystemen unter Praxisbedingungen.

Poster im Rahmen des DGKH- Kongresses, Berlin 20. - 23. April 2008 b

Abb.3:

Ansicht einer präparierten Petrischale als mikrobiologischer Teil der Testeinheit

zu Abb.3:

Ansicht einer präparierten Petrischale als mikrobiologischer Teil der Testeinheit

(zu erkennen ist der auf den Nährkarton aufgenähte Membranfilter)

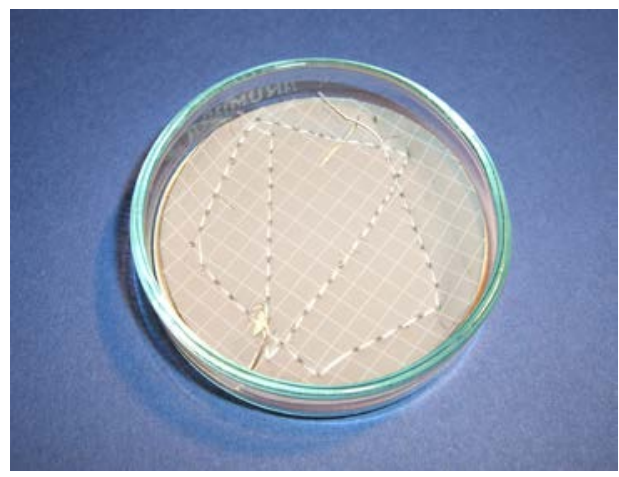




\subsubsection{Aktivierungsteil der Testeinheit}

Zur Herstellung der zweiten Komponente der Testeinheit wurde ein Reagenzglas mit $9 \mathrm{ml}$ destilliertem Wasser befüllt, welches durch Einschmelzen über dem Gasbrenner verschlossen wurde. Die Volumenangabe von $9 \mathrm{ml}$ hatte sich in Vorversuchen im Institut für Hygiene und Umweltmedizin in Göttingen (im Rahmen der 2006 durchgeführten Versuche in den Kornhäusern) als besonders geeignet erwiesen. Die so produzierte Glasampulle wurde im nächsten Schritt autoklaviert, bevor sie in den Silikonschlauch eingeführt wurde. Ein passender Silikonstopfen sorgte für den Verschluss des hinteren Teils des Silikonschlauches, während am vorderen Ende eine über dem Gasbrenner gezogene Glaskanüle eingebracht wurde. Diese speziell produzierte Auslauföffnung wurde durch das Weichschmelzen der offenen Seite eines Reagenzglases und den gleichmäßigen axialen Zug an dieser Seite in Form eines verjüngenden Glasrohrs geformt.

Die an der vorderen Spitze der Testeinheit entstehende Auslauföffnung wurde in der Gesamtanordnung der Testeinheit so platziert, dass diese in die Petrischale greift. Vor der Auslauföffnung installierte Glaswolle verhindert beim Öffnungsimpuls der Glasampulle ein Überfluten des Nährkartons mit autoklaviertem, destilliertem Wasser.

Abb.4:

Darstellung der erforderlichen Komponenten zur Aufnahme des Wasserreservoirs der Testeinheit

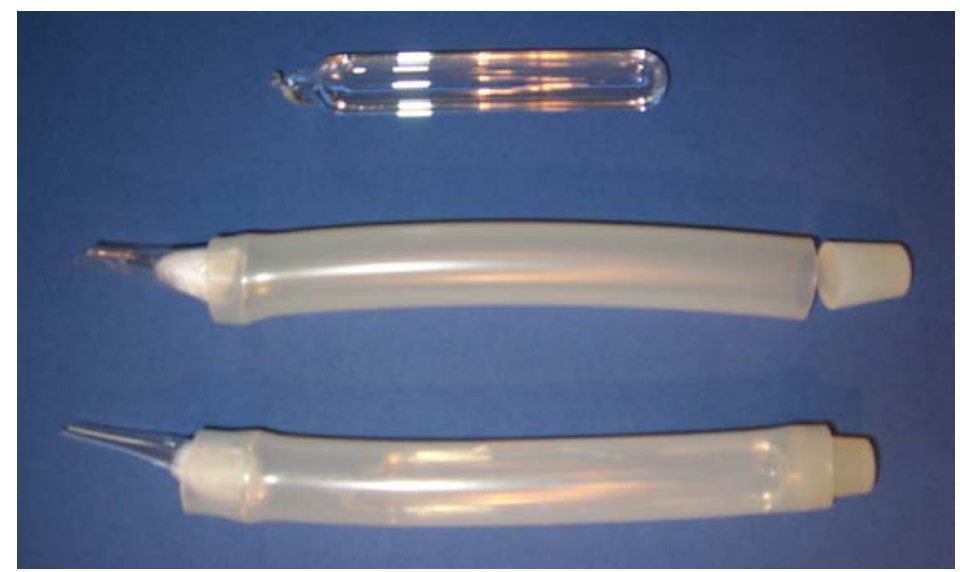

zu Abb.4:

Darstellung der erforderlichen Komponenten zur Aufnahme des Wasserreservoirs der Testeinheit

1. Oben: mit destilliertem Wasser zur Aktivierung der Testeinheit befülltes und verschlossenes Reagenzglas

2. Mitte: Stopfen, Silikonschlauch und Auslauföffnung

3. Unten: Zusammengesetzte Aktivierungseinheit, aus den gerade erwähnten Komponenten 
Abb.5:

Schematische Abbildung der gesamten Testeinheit im Querschnitt

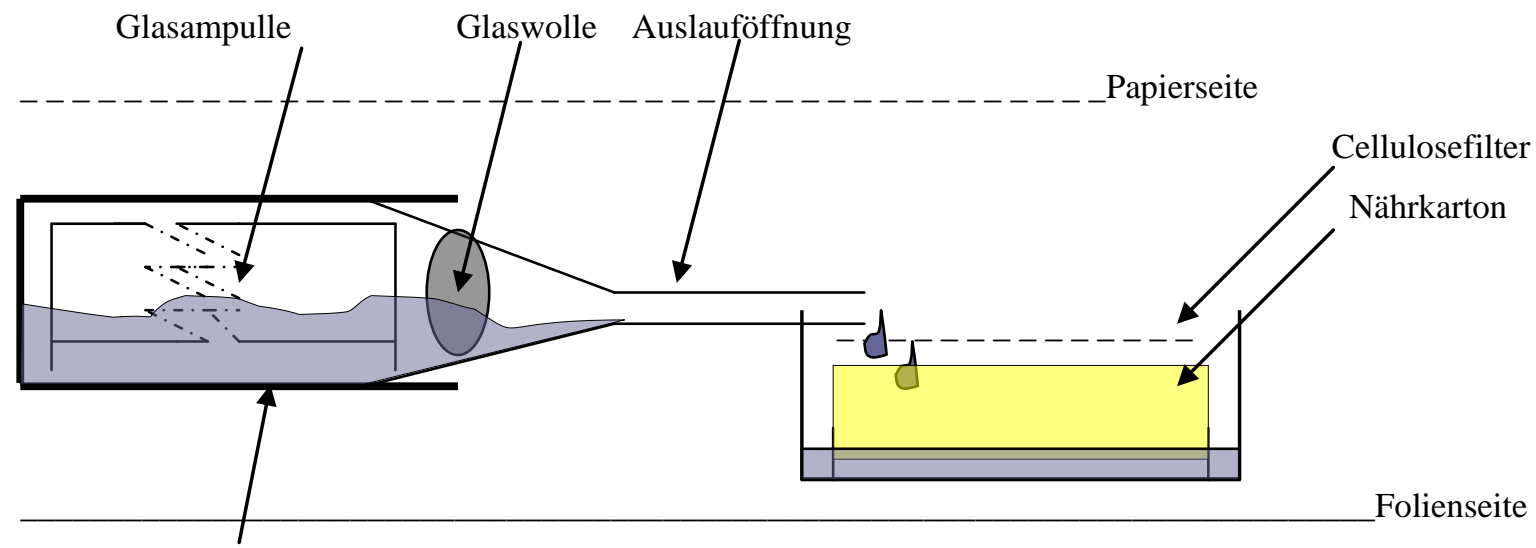

Siliconschlauch

zu Abb.5:

Schematische Abbildung der gesamten Testeinheit im Querschnitt

(der Öffnungsimpuls wurde hier bereits ausgelöst und das Wasser kann den biologischen Anteil, die Nährkartonscheibe, aktivieren)

modifiziert nach Dunkelberg und Schmelz (2008 b)

Testkit zur routinemäßigen Überwachung der Barriereeigenschaften von Sterilgutverpackungssystemen unter Praxisbedingungen.

Poster im Rahmen des DGKH- Kongresses, Berlin 20. - 23. April 2008 b

Abb.6:

Testeinheit in zu prüfender Sterilgutverpackung, fertig zur Verwendung

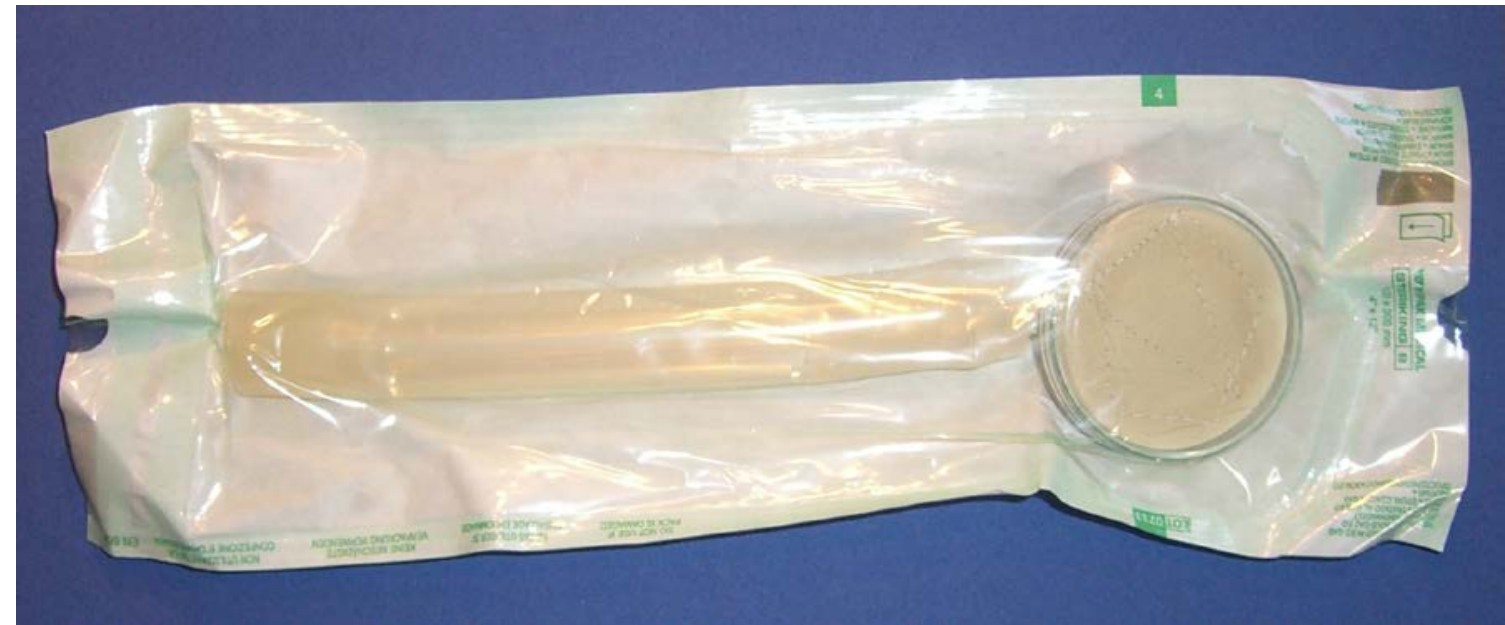

zu Abb.6:

Testeinheit in zu prüfender Sterilgutverpackung, fertig zur Verwendung

Der Nährkarton ist der Papierseite der Sterilgutverpackung zugewandt. Die Auslauföffnung greift pipettenartig in die Petrischale 


\subsection{Handhabung und Anwendung der Testeinheit}

Da im Rahmen der durchgeführten Untersuchungen die Barriereeigenschaften von „Papier zur Herstellung von Papierbeuteln“ und „,zur Herstellung von Klarsichtbeuteln und -schläuchen“ zur Verwendung als „,Verpackungen für in der Endverpackung zu sterilisierende Medizinprodukte“ überprüft werden sollten (DIN EN 868-3 (2009) S.1), musste auch die entwickelte Testeinheit in einer Papier-FolienVerbundverpackungseinheit eingepackt werden.

Somit bestand nunmehr zu den üblichen Verpackungseinheiten lediglich der Unterschied, dass nicht das Sterilgut, sondern die dargestellten Komponenten der Testeinheit in der Verpackung enthalten waren. Diese Papier-Folien-Verbundverpackungen mussten so verschlossen werden, dass „,[b]ei der Sichtprüfung mit normaler oder korrigierter Sehstärke im Durchlicht“ keine „Risse[...], Falten oder lokale[...] Verdickungen“ erkennbar sind, welche die Zweckmäßigkeit der Verpackung mindern könnten (DIN EN 868-3 (1999) S.3). Das Verschließen der Verpackungen erfolgte durch das Heißsiegelverfahren.

Im Folgenden schloss sich die Sterilisation, die Lagerung und die Distribution des Sterilgutes an. In diesem Verlauf wurden die Testeinheiten so behandelt, wie es auch bei anderen sterilisierten Medizinprodukten gehandhabt wird. Daher waren für die Testeinheiten die gleichen Voraussetzungen gegeben, wie sie bei gewöhnlichem Sterilgut vorliegen, das im medizinischen Alltag verwendet wird.

Auf diese Weise war es bei ausreichender Stückzahl der Testeinheiten nach Transport, Lagerung, Distribution und Auswertung möglich, eine Aussage über die Wahrung der Sterilität des in der Packung enthaltenen Sterilgutes zu treffen.

Zur Auswertung einer Testeinheit wurde die Glasampulle, ohne die Verpackung zu öffnen, mit einer Spindelpresse durch radialen Druck vorsichtig zerbrochen und die freigesetzte Wasserfüllung wurde über die Auslauföffnung in die Petrischale mit dem Nährkarton geleitet. Ein übermäßiges Überfluten des Nährkartons beim Öffnungsimpuls konnte durch eingesetzte Glaswolle und den pipettenartigen Aufbau vermieden werden. Das Zerbrechen der Glasampulle stellte keine Gefahr für die Unversehrtheit der Verpackung dar, da das zerbrochene Glas durch den Silikonschlauch gesichert war.

Durch den entscheidenden Schritt der Befeuchtung der Nährkartonscheibe wurde der bis jetzt inaktive mikrobiologische Teil der Testeinheit aktiviert. Sollten Mikroorganismen im Rahmen der Handhabung der Verpackungen (Umgebungskeimbelastung oder mögliche Druckbeaufschlagung durch Transport) nach der Sterilisation durch die Verpackung eingedrungen sein, so könnten diese auf den Membranfilter sedimentieren, proliferieren und auf diese Weise makroskopisch sichtbare Kolonien ausbilden, die eine Unsterilität indizieren.

\subsection{Kritische Begutachtung der produzierten Testeinheit}

Um bei den durchgeführten Testungen ein aussagekräftiges Ergebnis erzielen zu können, war es obligat, die entwickelte Testeinheit und alle dazugehörigen Elemente auf fehlerhafte Produktion oder minimale Fehlerquellen zu untersuchen. Im Rahmen dieser Überlegungen wurde eine Reihe an Methoden herausgearbeitet, mit denen die Testeinheit adäquat überprüft werden konnte. Dies betrifft sowohl die Güte der Verpackung, als auch den biologischen Anteil der Testeinheit und den Aktivierungsteil. 


\subsubsection{Güte des Verpackungssystems}

Zunächst war es von entscheidender Bedeutung, die richtige Größe zum Verpacken der Testeinheiten zu ermitteln, um eine Beschädigung der Verpackung durch zu straffen Sitz aber gleichzeitig auch durch eine zu lockere Fixierung der Komponenten der Testeinheit zu verhindern. Zugleich war es hierbei wichtig, immer die optimale Verpackungsgröße für das zu sterilisierende Objekt auszuwählen, um das Volumen im Packungsinneren möglichst gering zu halten. Denn je mehr Volumen die Papier-Folien-Verbundverpackung enthält, umso mehr Volumenstrom kann hier (zum Beispiel durch Druckschwankungen) hervorgerufen werden. Zudem wurde bei der Anschaffung der Sterilgutverpackungen darauf geachtet, dass in allen Fällen die gleichen Verpackungshersteller gewählt wurden. Dies erlaubte eine exakte Vergleichbarkeit der bei den verschiedenen Chargen genutzten Verpackungen. Da es sich in Oldenburg um eine Lagerhalle verschiedener Sterilgüter ohne bevorzugte Verpackungslieferanten handelt, wurde auch hier die für die anderen Teststandorte gewählte Sterilgutverpackung zum Einsatz gebracht, um eine bessere Vergleichbarkeit der resultierenden Ergebnisse untereinander zu erhalten.

Da diese Testung der Verpackungssysteme nicht in erster Linie eine Prüfung der Schweißsiegelnaht anbelangte, wurde diese, wie es auch häufig im klinischen Alltag durchgeführt wird, doppelt angelegt, um so eine Kontaminationsgefahr durch eine fehlerhafte Verschweißung zu minimieren (fehlerhaft im Bezug auf Faltenbildung oder unzureichende Verschweißung). Sterilgutverpackungen mit schadhafter Verschweißung im Sinne geschmolzener Folienabschnitte oder Defekte der Folie aufgrund zu heißer oder lang anhaltender Siegelung wurden sofort aussortiert. In diesem Fall hätte auch eine doppelte Verschweißung keinen Sinn.

Ansonsten wurde bei der Siegelung darauf geachtet, dass gemäß DIN EN ISO 11607-2 weder „Kanalbildung oder offene Siegelnähte“, noch „Durchstiche oder Risse“ vorlagen. Zudem sollte eine „intakte Siegelung bei einer festgelegten Siegelungsbreite“, nicht jedoch „Delaminierung oder Ablösung von Materialien“ zu erkennen sein (DIN EN ISO 11607-2 (2006) S.11) .

Im Falle eines mikrobiologischen Nachweises einer Rekontamination einer Testeinheit bestand die Möglichkeit, die Unversehrtheit und Dichtigkeit der Schweißsiegelnaht durch den „dye penetration test“ (auch Schweißsiegelnahttest) zu überprüfen und somit falsch positive Ergebnisse auszusortieren. Der innere Rand der Siegelnaht der zu untersuchenden Verpackung wurde mit einer geringen Menge farbiger Prüflösung befüllt, welche sich durch kapillare Kräfte in Undichtigkeiten der Siegelnaht zieht und somit diese für den Betrachter anfänglich unsichtbaren Defekte optisch detektierte (genaue Erläuterungen siehe Abschnitt 4.4, Seite 71).

\subsection{2 Überprüfung des Aktivierungsteils}

Bei der Konstruktion der Testeinheiten wurde besondere Acht darauf gegeben, dass die mit destilliertem Wasser gefüllten Reagenzgläser bereits einmal autoklaviert wurden, bevor sie der späteren Testeinheit zugeführt wurden, um unvollständig zugeschmolzene oder zu dünnwandige Gläser, die dem Druck im Autoklaven nicht standhalten könnten, auszusortieren. Außerdem kam der eingefüllten Wassermenge eine entscheidende Bedeutung zu, da nach späterem Eröffnen und Befeuchten der Nährkartonscheibe ein ausreichendes Wasserreservoir gegeben sein musste, um eine notwendige Durchnässung zu ermöglichen und somit ein Wachstum von eingedrungenen Keimen sicherzustellen. Durch das Einbringen von Glaswolle in die langgezogene Auslauföffnung wurde ein übermäßiges Überschwemmen des Nährkartons und somit ein Wegspülen sedimentierter Keime vermieden. 
Zudem verhinderte die Glaswolle ein Verstopfen der Auslauföffnung durch beim Zerbrechen der Glasampulle entstandene Glassplitter. Eine extra für diese Zwecke modifizierte Schraubzwinge ermöglichte den Öffnungsimpuls des Reagenzglases, ohne eine Beschädigung durch mechanische Beanspruchung an der Verpackung hervorzurufen. Für eine einwandfreie Nutzung der Testeinheit war es unerlässlich, sich in der Handhabung selbiger zu üben und sie zu optimieren, was im Rahmen der Kontrollgruppe erfolgte. Es wurden 200 Kontrollproben (Lagerung im Institut für Hygiene und Umweltmedizin in Göttingen) angelegt, eingelagert und anschließend ausgewertet (100 Testeinheiten: Kontrollprobe 1 (Vorversuche 2006) und 100 Testeinheiten: Kontrollprobe 2 (2008)).

\subsection{3 Überprüfung der Anzüchtbarkeit verschiedener Bakterienspezies durch die verwendeten Nährkartonscheiben}

Im Rahmen der Vorversuche in den Kornhäusern wurde die Autoklavierbarkeit der angewandten Nährkartonböden ohne Verlust der Kultureigenschaften nachgewiesen (siehe Abschnitt 1.7, Seite 17) und konnte somit auf die in Bad Rothenfelde und Oldenburg durchgeführten Versuchsreihen übertragen werden. Bei den Testkeimen handelte es sich um natürlicherweise in der Umgebungsluft aber auch in Räumlichkeiten mit Reinraumbedingungen (zum Beispiel im Operationssaal) vorhandene Arten. Folgende Keime konnten unter Reinraumbedingungen ermittelt werden (Moggio et al. 1979) und spielten somit auch für diese Studie im Hinblick auf die im Operationsbereich von Bad Rothenfelde gelagerten Chargen eine Rolle.

Tabelle 2:

Die in der Luft eines Operationssaales nachgewiesenen Keimarten (grobe Zuordnung)

\begin{tabular}{|l|l|l|}
\hline \multicolumn{1}{|c|}{$\begin{array}{c}\text { Untersuchte Gesamtkeimzahl } \\
\text { n= 12666 Keime }\end{array}$} & \multicolumn{1}{c|}{ Gramfärbung } & \\
\hline Mikrokoke Zuordnung & & 36,2 \\
Diphtheroide Stäbchen & Positiv & 22,6 \\
Bazillen & Positiv & 18,4 \\
Staphylococcus epidermidis & Positiv & 13,7 \\
Staphylococcus aureus & Positiv & 0,3 \\
Vibro extorquens & Positiv & 2,6 \\
Pseudomonas & Negativ & 0,04 \\
Serratia marcescens & Negativ & 0,02 \\
Klebsiellen & Negativ & 0,01 \\
Neisserien & Negativ & 0,01 \\
Pilze & Negativ & 5,9 \\
\hline
\end{tabular}

Moggio et al 1979 - Wound infections in patients undergoing total hip arthroplasty. Ultraviolet light for the control of airborne bacteria.

Arch Surg 1979; 114 (7): 815 - 823 


\subsubsection{Kontrollgruppe}

Nachdem nun die Einzelkomponenten der Testeinheit begutachtet und kontrolliert wurden, war nicht zu vernachlässigen, die Testeinheit auch als „Gesamtwerk“ im Rahmen dieser Arbeit nochmals zu überprüfen.

Hierzu wurden weitere 100 Testeinheiten konstruiert, allein um eine repräsentative Prüfung der Testsysteme und eine Aussage über deren einwandfreie Funktionstüchtigkeit und eine fehlerfreie Handhabung zu erlauben. Selbstverständlich wurden diese Testeinheiten (Kontrollgruppe 2) genauso präpariert und autoklaviert wie die später eingesetzten weiteren 750 Einheiten. Gleichzeitig war hiermit die Möglichkeit gegeben, die Handhabung zu verbessern und den Gebrauch der Testeinheiten versiert durchzuführen. Außerdem wurden bei diesem Durchlauf mit den Kontrollproben Alternativen aufgezeigt, die zur Optimierung des zeitlichen (Planung der Rückführungsetappen) wie auch des räumlichen Managements (Präparation der Brutschränke) dienten. Dieser zusätzliche Arbeitsschritt diente also lediglich der Übung im Umgang mit der Aktivierung der Testeinheit, ohne die Verpackung zu beschädigen und eine angemessene, regelmäßige Befeuchtung der Nährkartonscheibe herauszuarbeiten.

Das gleiche Procedere erfolgte bereits für die Vorversuche im Rahmen der Lagerung von 381 Testeinheiten in den Kornhäusern im Jahr 2006. Auch hier wurden im Voraus 100 Kontrollproben (Kontrollgruppe 1) für die Lagerung im Labor und die Übung in der Handhabung angelegt.

\subsection{Konzept der Lagerung}

Es wurde eine Reihe Testeinheiten (850 Stück inklusive Kontrollgruppe) angefertigt, um sie anschließend an geeigneten Standorten praxisbezogen zu deponieren. Hierzu wurde die Herzklinik in Bad Rothenfelde ausgewählt, wo Reinraumbedingungen herrschten. Als Abstufung zu den dort herrschenden Reinraumbedingungen wurde als Referenz ein weiterer Lagerungsort, eine Lagerhalle für Sterilgüter in Oldenburg, bestimmt. Hier lagen keine klimatisierten Reinraumbedingungen vor, es handelt sich eher um eine gewöhnliche Halle mit sichtbarer Oberflächenbeschmutzung und Verkehrsbetrieb. Dieser Standort wurde gewählt, um eine gewisse Graduierung zu den Reinraumbedingungen in Bad Rothenfelde und den bereits 2006 erfolgten Testungen in den Kornhäusern zu schaffen, wo eine sichtbare staubige und keimbeladene Luft zu finden war.

Die Rückführung und Auswertung der Testsysteme erfolgte in Etappen, um den Faktor der Lagerungsdauer im Hinblick auf eine mögliche Kontamination der Einheiten an den verschiedenen Lagerungsstandorten feststellen zu können. 
Die Testeinheiten wurden in Kohorten auf zwei Standorte verteilt:

o Schüchtermann - Klinik Bad Rothenfelde (Reinraum-Bereich; geringe Luftkeimzahl)

o Sterilgutlager des Klinikums Oldenburg (Lagerhalle mit Verkehrsaufkommen)

\section{Bad Rothenfelde und Oldenburg}

Bei der Lagerung der Testeinheiten wurde hier besonderer Wert auf die Örtlichkeiten gelegt, an denen diese deponiert wurden. Hierzu wurde der Operationsbereich in der Herzklinik Bad Rothenfelde ausgewählt, wo Reinraumbedingungen durch raunlufttechnische Anlagen vorherrschen. Eine weitere Abstufung zu dem voran geschilderten „Worst-Case-Szenario“ (siehe Abschnitt 1.7, Seite 16 - 19) wurde geschaffen, indem als zweite Örtlichkeit eine Lagerhalle für Sterilgüter in Oldenburg genutzt wurde. Somit war ein direkter Vergleich zwischen der Lagerung unter Reinraumbedingungen (Bad Rothenfelde), „gewöhnlicher“ Umgebungskeimlast (Oldenburg) und besonders hoher Keimlast und staubiger Luft (Kornhäuser) möglich.

Die zwei Kohorten an Testeinheiten wurden an den Bestimmungsorten in fixen Positionen deponiert. Dabei wurde die Kohorte, welche in Bad Rothenfelde eingelagert wurde, nochmals auf verschiedene Bereiche innerhalb des Operationsbereiches aufgeteilt. Ein Teil der Testeinheiten wurde auf dem Sterilflur deponiert und zwar zur Hälfte in einem verschließbaren Schrank, der Rest in einem offenen Regal. Der andere Teil der Testeinheiten wurde auf Station 8 in einem geschlossenen Schrank platziert und gelagert. Diese in Bad Rothenfelde an verschiedenen Plätzen deponierten Testeinheiten konnten in folgenden Berechnungen aufgrund sich entsprechender Resultate (hervorgerufen durch sehr ähnliche Lagerungsbedingungen) und zur besseren Übersicht zusammenfassend als eine Charge betrachtet werden.

Da in der Lagerhalle in Oldenburg keine unterteilten Räumlichkeiten existieren, konnte hier auf eine weitere Gruppierung der Testeinheiten verzichtet werden, sodass hier die gesamte Kohorte während der gesamten Expositionszeit an einem einzigen Platz verblieb.

Bei diesem Expositionsversuch in der Herzklinik und der Lagerhalle erfolgte nach bestimmten Zeitabständen in drei Etappen die Rückführung einer definierten Anzahl an Testeinheiten, die anschließende Aktivierung, Befeuchtung und die nachfolgende Auswertung hinsichtlich einer potenziellen Rekontamination des Sterilgutes. Bad Rothenfelde wurde im Rahmen der Untersuchungen mit insgesamt 450 Testeinheiten bestückt, wobei die einzelne Rückführungsetappe mit jeweils 150 gelagerten Testeinheiten zu beziffern ist.

Die Stückzahl der in Oldenburg gelagerten Testeinheiten betrugt 300. Hierbei erfolgten die Rückführungsetappen der Testsysteme in Hunderterschritten.

Als Kontrollgruppe 2 dieser Versuchsreihe wurden weitere 100 Testeinheiten im Institut für Hygiene und Umweltmedizin in Göttingen deponiert. Diese Testeinheiten wurden vor Beginn der Rückführung der Testeinheiten aus Bad Rothefelde und Oldenburg (d. h. nach einer Lagerung von 2 Monaten) ausgewertet, um den Umgang mit den Testsystemen zu routinieren. 
Tabelle 3:

Lagerungsorte und -zeiten der Testeinheiten im Überblick

\begin{tabular}{|l|c|}
\hline Lagerungsort & Expositionsdauer \\
\hline Bad Rothenfelde & $2 \frac{1}{2}$ Monate \\
Oldenburg & 6 Monate \\
& 8 Monate \\
Kontrollgruppe 2 & $2 \frac{1}{2}$ Monate \\
& 5 Monate \\
\hline
\end{tabular}

\subsection{Einflussfaktoren an den verschiedenen Standorten}

\subsubsection{Einschätzung der Umgebungskeimlast}

Tabelle 4:

Schätzwerte für die Anzahl der in der Luft enthaltenen Keimlast an den verschiedenen Lagerungsstandorten

\begin{tabular}{|c|c|}
\hline Lagerungsstandorte der Testeinheiten & 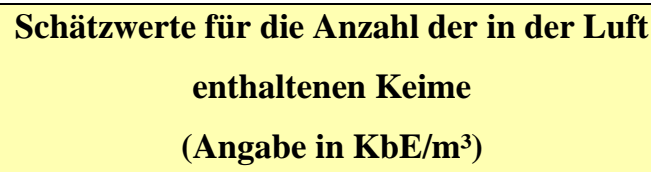 \\
\hline Bad Rothenfelde & 150 \\
\hline Kontrollgruppe 2 (Labor) & 350 \\
\hline Oldenburg & 350 \\
\hline
\end{tabular}

\section{Bad Rothenfelde}

Nach Angaben der Arbeitsgemeinschaft der Wissenschaftlichen Medizinischen Fachgesellschaften (AWMF) „gelten für desinfizierte OP- Räume ohne OP- Betrieb“ (URL http://www.uni-duesseldorf.de/awmf/ll/029-

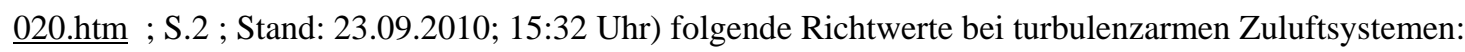

\section{- Partikelkonzentration}

(als Partikel gelten jene mit einem Durchmesser $>0,5 \mu \mathrm{m} / \mathrm{m}^{3}$ )

Der Richtwert liegt bei $\underline{4000}$, während der Grenzwert bei 10.000 liegt.

\section{- Luftkeimkonzentration}

(Messung erfolgt in der Einheit $\mathrm{KbE} / \mathrm{m}^{3}$ )

Der Richtwert beträgt $\underline{4}$ und der Grenzwert liegt bei $\underline{10}$. 
„Wichtiger, aber bisher nicht geregelt, sind Prüfungen während des laufenden Betriebs der RLTA. Derzeit liegt diese Prüfung in der Entscheidung des Hygienikers“

(URL http://www.uni-duesseldorf.de/awmf/ll/029-020.htm ; S.2 ; Stand: 23.09.2010; 15:32 Uhr).

Diese Werte gelten jedoch nur für die Zuluft aus der Raumlufttechnischen Anlage, nicht jedoch für die Raumluft während laufenden OP- Betriebes. Je nach der Anzahl der im Operationssaal befindlichen Personen werden durch Bewegungen, durch Sprechen, Niesen , Husten oder einfach durch kleine Hautschuppen ständig Keime in die OP-Luft befördert. Daher waren diese Richt- und Grenzwerte auf den Lagerungsstandort Bad Rothenfelde nicht direkt übertragbar.

Abhängig von der Personenanzahl und ihrem Aktivitätszustand (sprechen, husten, gehen...) konnte hier von einer Keimbelastung während der Operation von 50 - $200 \mathrm{KbE} / \mathrm{m}^{3}$ ausgegangen werden.

Für den OP-Bereich in Bad Rothenfelde wurde im Rahmen dieser Arbeit eine Keimbelastung der Luft von 150 $\mathrm{KbE} / \mathrm{m}^{3}$ angenommen.

\section{Kontrollgruppen und Oldenburg}

Die Bestimmung der Luftkeimzahl der äußeren Umgebungsluft in Göttingen und die Sammlung verschiedener Messdaten erfolgte im Rahmen innenraumlufttechnischer Erhebungen und ergab eine approximative Luftkeimzahl von $350 \mathrm{KbE} / \mathrm{m}^{3}$.

Dieser Wert wurde auch auf die Verhältnisse in Oldenburg übertragen.

\subsubsection{Determinante: Temperaturschwankungen}

Darstellung der Temperaturschwankungen während des Expositionsintervalls der Testeinheiten der Sterilgutverpackungssysteme in Bad Rothenfelde

Da bei der Umgebung der in Bad Rothenfelde gelagerten Testeinheiten ausschließlich klimatisierte Verhältnisse vorlagen, war hier nicht mit besonders großen täglichen Temperaturschwankungen zu rechnen. Um Berechnungen trotzdem zu ermöglichen, wurde eine geringe tägliche Temperaturdifferenz von $1^{\circ} \mathrm{C}$ angenommen (unter Innenraumbedingungen von $20^{\circ} \mathrm{C}$ ).

Gleiches gilt für die im Institut für Hygiene und Umweltmedizin gelagerte Kontrollgruppe 2, auch hier handelte es sich um Innenräume von $20^{\circ} \mathrm{C}$ mit geringen Temperaturschwankungen von $1{ }^{\circ} \mathrm{C}$. Auf diese Weise konnte das Charles'sche Gesetz auch hier Anwendung finden.

\section{Darstellung der Temperaturschwankungen während des Expositionsintervalls der Testeinheiten der Sterilgutverpackungssysteme in der Lagerhalle in Oldenburg}

Bei den Temperaturschwankungen der in Oldenburg gelagerten Testeinheiten stellte sich dies etwas anders dar. Hier handelte es sich nicht um klimatisierte Räumlichkeiten. Die Verpackungen waren hier mehr oder minder den Umgebungstemperaturen ausgesetzt. Zwar lagen sie auch hier in einer Lagerhalle geschützt, um eine Berechnung des Volumenstroms zu ermöglichen, wurden hier jedoch zur Vereinfachung die schwankenden 
Außentemperaturen, die den Temperaturen in der Lagerhalle weitgehend entsprachen, als Anhaltspunkte herangezogen. Die nächstgelegene Wetterstation befindet sich laut Deutschem Wetterdienst im 39 Kilometer entfernten Bremen (Bundesministerium für Verkehr, Bau und Stadtentwicklung - Deutscher Wetterdienst (o.J.) URL www.dwd.de).

\subsubsection{Determinante: atmosphärische Druckschwankungen}

Einwirkende Druckdifferenzen auf die in Oldenburg und Bad Rothenfelde gelagerten Sterilgutverpackungseinheiten

Zur Berücksichtigung witterungsbedingter atmosphärischer Luftdruckschwankungen wurden Daten des Deutschen Wetterdienstes herangezogen. Da es bei dem Deutschen Wetterdienst keine Daten für die Standorte Bad Rothenfelde und Oldenburg gab, wurden hier die Daten von anderen, in direkter Nähe zu den beiden Standorten befindlichen Wetterstationen mit dokumentierten Messwerten der wetterbhängigen atmosphärischen Luftdruckschwankungen verwendet. Demnach wurden für Bad Rothenfelde entsprechend die Daten der Wetterstation in Münster - Osnabrück (ca. 18 km entfernt von Bad Rothenfelde) und für Oldenburg die Daten der Wetterstation in Bremen (ca. 39 km entfernt von Oldenburg) angenommen (Bundesministerium für Verkehr, Bau und Stadtentwicklung - Deutscher Wetterdienst (o.J.) URL www.dwd.de).

Für die Berechnungen wurden hier die Werte der während der Expositionsdauer durchlaufenen witterungsbedingten Luftdruckänderungen betrachtet, da diese zu einem Volumenstrom ins Verpackungsinnere führen. Durch den Anstieg des atmosphärischen Luftdrucks resultiert eine Abnahme des Volumens im Packungsinneren, die im Falle einer gasdurchlässigen, aber in der Form weitgehend starren Verpackung zum Volumenausgleich durch Lufteintritt führen würde.

Dieser Volumenstrom konnte mithilfe des Boyle- Mariotte- Gesetzes ermittelt werden.

2.8 Berechnung der Volumenströme ins Verpackungsinnere bei bekannten Druck- und Temperaturverhältnissen

Bei Betrachtung der allgemeinen Gasgleichung $\left(p^{*} V=R^{*} T\right)$ kann das Volumen im wesentlichen durch zwei Größen beeinflusst werden:

\section{A. Druckänderungen (linker Term der Gleichung):}

Bei definiertem Volumen führt eine in der Regel geodätisch bedingte Druckänderung (zum Beispiel im Rahmen des Transportes in Aufzügen oder Flugzeugen) ebenfalls zu einer Veränderung des Volumens.

Das Boyle- Mariotte- Gesetz wird zur Berechnung der druckbedingten Volumenveränderung bei konstanter Temperatur verwendet.

$$
\begin{aligned}
& \text { Für die Gleichung gilt: } \\
& \mathrm{p}=\text { Druck } \\
& \mathrm{V}=\text { Volumen } \\
& \mathrm{R}=\text { Gaskonstante } \\
& \mathrm{T}=\text { Temperatur }
\end{aligned}
$$




\section{B. Temperaturänderung (rechter Term der Gleichung):}

Bei definiertem Volumen in der Sterilgutverpackung führt eine Temperaturänderung zu einer Volumenänderung. Die Berechnung des temperaturabhängigen Volumens (bei konstantem Druck) erfolgt durch das Charles`sche Gesetz.

Kann die Volumenänderung nicht ausgeglichen werden (geschlossenes System), so resultiert eine Druckänderung. Bei Papier-Folien-Verbundverpackungen ist ein Volumenausgleich jedoch direkt möglich (die Verpackungen müssen eine Gasdurchlässigkeit aufweisen), sodass unmittelbar eine Volumenänderung resultiert. Die Volumenänderung geht mit einem Volumenstrom, das heißt einem Luftfluss, einher.

\subsubsection{Berechnung des druckabhängigen Volumenstroms durch die Papierseite der Testeinheit (Boyle- Mariotte- Gesetz)}

Anhand der Messwerte (der in Abschnitt 2.7.3, Seite 32 aufgeführten Wetterstationen) konnte der aufgrund wetterabhängiger atmosphärischer Druckschwankungen verursachte Volumenstrom durch die Sterilgutverpackung für jede Kohorte ermittelt werden. Die während der Expositionsdauer erfolgten witterungsbedingten Luftdruckanstiege dienten der Berechnung der daraus resultierenden Volumenströme ins Verpackungsinnere. Denn nur ein Luftdruckanstieg führte zu einem ins Verpackungsinnere gerichteten Luftstrom.

Das approximative Volumen der Sterilgutverpackung der Testeinheit betrug ca. $240 \mathrm{ml}$. Dieses Volumen wurde in der Praxis durch die Gasphase Luft gebildet.

Die Temperatur (T) wurde im Rahmen folgender Berechnungen als konstant betrachtet.

\section{Es gilt somit:}

- $\mathrm{T}=$ const.

- $\quad \mathrm{p} \sim 1 / \mathrm{V}$

- $\mathrm{p}_{1} / \mathrm{p}_{2}=\mathrm{V}_{2} / \mathrm{V}_{1}$

- $\mathbf{V}_{2}=\left(\mathbf{p}_{1} * \mathbf{V}_{1}\right) / \mathbf{p}_{2}$

Bedingungen:

- $\mathrm{p}_{1}=$ Luftdruck (Anfangswert in $\mathrm{hPa}$ )

- $\mathrm{p}_{2}=$ Luftdruck (Endwert nach Luftdruckanstieg in $\mathrm{hPa}$ )

$-\mathrm{V}_{1}=$ Volumen (Anfangswert in $\mathrm{ml}$ )

- $\mathrm{V}_{2}=$ Volumen (Endwert nach Druckanstieg in $\mathrm{ml}$ )

[Druck- und Temperaturdaten von URL www.wetterstation-goettingen.de und Bundesministerium für Verkehr, Bau und Stadtentwicklung - Deutscher Wetterdienst (o.J.) URL www.dwd.de) ] 


\subsubsection{Berechnung der Volumenänderung in Abhängigkeit von der Temperaturdifferenz bei bekanntem Ausgangsvolumen (Charles`sches Gesetz)}

Zur Berechnung des durch die Temperaturschwankungen hervorgerufenen Lufteinstroms in die Papier-FolienVerbundverpackung wurden die Daten des jeweiligen Standortes (siehe Abschnitt 2.7.2, Seite 31 - 32) herangezogen - sei es bei klimatisierten Räumlichkeiten die Annahme von einer täglichen Temperaturdifferenz von $1^{\circ} \mathrm{C}$ oder aber die den Standorten der Kornhäuser (Vorversuche 2006) und der Lagerhalle in Oldenburg entsprechenden Außentemperaturen. Bei Annahme konstanter Druckverhältnisse und einem Volumen des Verpackungsinneren von $240 \mathrm{ml}$ konnte mithilfe des Charles'schen Gesetzes der durch die Temperaturschwankungen verursachte Volumeneinstrom ins Innere des Sterilgutverpackungssystems berechnet werden. Hierbei führte lediglich ein Temperaturabfall zu einem Luftvolumeneinstrom ins Verpackungsinnere.

\section{Es gilt somit:}

- $\quad$ Bei $\mathrm{p}=$ const. $\left(\mathrm{p}_{0}=\right.$ mittlerer atmosphärischer Luftdruck)

$$
\begin{gathered}
\mathrm{dV}_{2} /\left(\mathrm{V}_{1} * \mathrm{dT}_{2}\right)=\mathrm{R} /\left(\mathrm{p}_{0}=\mathrm{V}_{1}\right)=\gamma=\text { const. }=0,0036 / \mathrm{K} \\
\int_{V 1}^{V 2} d V 2 / V 2=\int_{T 1}^{T 2} \gamma d V 2
\end{gathered}
$$

\section{Lösung des Integrals:}

$$
\mathrm{V}_{2}=\mathrm{V}_{1} *\left(1+\gamma\left(\mathbf{T}_{2}-\mathrm{T}_{1}\right)\right)
$$

Bedingungen:

$-\mathrm{V}_{1}=$ Ausgangsvolumen einer Sterilguteinheit $=240 \mathrm{ml}$

- $\mathrm{V}_{2}=$ Endvolumen (nach Temperaturabfall in $\mathrm{ml}$ )

- $\mathrm{T}_{1}=$ maximaler Tagestemperaturwert (in Kelvin)

- $\mathrm{T}_{2}=$ minimaler Tagestemperaturwert (in Kelvin)

$-\gamma=$ Volumenausdehnungskoeffizient $=0,0036$

[Druck- und Temperaturdaten von URL www.wetterstation-goettingen.de und Bundesministerium für Verkehr, Bau und Stadtentwicklung - Deutscher Wetterdienst (o.J.) URL www.dwd.de) ] 
Im Rahmen des Feldversuches dieser Arbeit (Bad Rothenfelde, Oldenburg) konnten die Ergebnisse der Testungen aus den Kornhäusern einbezogen werden, indem anhand der Vorversuche auch für diese Testsysteme der Einfluss durch Temperaturschwankungen, atmosphärische Druckalterationen und die Umgebungskeimlast eingeschätzt wurde. Auch hier fand das Boyle- Mariotte- Gesetz und das Charles'sche Gesetz Anwendung, um den erfolgten Volumeneinstrom ins Verpackungsinnere zu ermitteln. Die Rekontamination in den Kornhäusern (von 55,2 \% bis zu 84,2 \%) konnte der Rekontamination der Einheiten aus Bad Rothenfelde und Oldenburg gegenübergestellt werden. Im Anschluss konnte die Abhängigkeit der Rekontamination von den Umgebungsbedingungen an den verschiedenen Standorten ermittelt werden.

\subsection{LRF (Logarithmischer Reduktionsfaktor)}

Da in den Kornhäusern eine extrem hohe Keimbelastung der Umgebungsluft vorzufinden war und auch eine recht hohe Zahl kontaminierter Testeinheiten zu verzeichnen war, schien es unumgänglich, sich mit der Effektivität der Barriereeigenschaften der Sterilgutverpackung zu beschäftigen. Daher war es von besonderer Bedeutung, unter Berücksichtigung des Sterilitätssicherheitswertes (SAL), eine genaue Einschätzung der Barrierewirkung der Papier-Folien-Verbundverpackung nach erfolgter Sterilisation vorzunehmen. Hierbei mussten natürlich die herrschenden Umgebungsbedingungen (Luftdruck, Temperatur, Luftkeimgehalt) und ihr Einfluss auf die Rekontamination der Testeinheiten in die Kalkulation einbezogen werden.

Bei bekanntem Luftvolumenstrom durch das Verpackungsmaterial (durch Druck- oder Temperaturdifferenzen hervorgerufen) und definiertem Keimgehalt der Umgebungsluft (in $\mathrm{KbE} / \mathrm{m}^{3}$ ) konnte eine berechenbare mikrobiologische Beanspruchung des verpackten Sterilgutes mit keimbeladener Luft ermittelt werden.

Demgegenüber stand der praktische Nachweis auf den im Verpackungsmaterial enthaltenen Nährkartons nachweisbaren Kolonien nach Inkubation.

Hieraus ergab sich eine Möglichkeit zur Bewertung der Effektivität der Barrierewirksamkeit der Papier-FolienVerbundverpackungen durch die Berechnung des LRF (= logarithmischer Reduktionsfaktor). Der LRF beschreibt auf diese Weise das Verhältnis einer mikrobiologischen Beanspruchung der Sterilgutverpackungen zur auf den integrierten Nährkartonscheiben nachweisbaren Rekontamination durch Mikroorganismen (Kolonien auf dem Nährmedium).

Rechnerische Darstellung des Logarithmischen Reduktionsfaktors:

$$
\begin{gathered}
\text { LRF }=\log \left(N_{0} / N_{1}\right) \\
\text { LRF }=\log N_{0}-\log N_{1}
\end{gathered}
$$


Gleichzeitig konnte die Filtereffektivität der Verpackung durch folgende Gleichung berechnet werden:

\section{Filterwirkung $(\%)=\left[1-\mathrm{N}_{1} / \mathrm{N}_{0}\right] * 100$}

\section{Für beide Gleichungen gilt:}

$\mathrm{N}_{0}=$ durch den Volumenstrom und den Luftkeimgehalt auf das Verpackungsmaterial wirkende mikrobiologische Beanspruchung während der Expositionsdauer

$\mathrm{N}_{1}=$ Rekontamination der in der Verpackung enthaltenen Nährkartonscheibe nach Exposition, Inkubation und abschließender Quantifizierung der gewachsenen Kolonien

Rechenbeispiel:

Bei einer mikrobiologischen Beanspruchung von $\mathrm{N}_{0}=100$ Mikroorganismen und einer nachweisbaren Kontamination des Nährmediums von $\mathrm{N}_{1}=1$ (Kolonie) ergibt sich laut dieser Berechnungen ein LRF von 2 und eine Filtereffektivität von $99 \%$.

\section{Berücksichtigung des Sterilitätssicherheitswertes (SAL = sterility assurance level):}

Der Sterilitätssicherheitswert von $10^{-6}$ wird gefordert, um ein Medizinprodukt als steril auszuweisen. Dies bedeutet, dass von 1 Million Sterilgütern max. 1 Keim auf einem der Sterilgüter vorhanden sein darf, damit die gesamte Charge noch als steril deklariert werden kann.

Demzufolge musste der berechnete LRF und die mikrobiologische Beanspruchung auf das entsprechende Verpackungssystem unter Berücksichtigung dieses Sterilitätssicherheitswertes betrachtet werden.

Somit wurde der geforderte $\mathrm{LRF}_{\text {erforderlich }}$ berechenbar, der benötigt wird, um die Sterilität des verpackten Medizinproduktes bei gegebener mikrobiologischer Beanspruchung $\left(\mathrm{N}_{0}\right)$ zu wahren.

Es gilt folgende Herleitung:

$$
\begin{gathered}
\log \mathrm{N}_{0}-\mathrm{LRF}_{\text {erforderlich }} \leq \log 10^{-6}(=\mathrm{SAL}) \\
-\mathrm{LRF}_{\text {erforderlich }} \leq-\log \mathrm{N}_{0}-\log 10^{-6} \\
\mathbf{L R F} \mathbf{F}_{\text {erforderlich }} \leq \log \mathbf{N}_{\mathbf{0}}+\mathbf{6}
\end{gathered}
$$


Mangels Keimnachweis in den Untersuchungen in Bad Rothenfelde und Oldenburg ließ sich der LRF allein aus den Daten zu diesen Standorten nicht ermitteln. Somit war hier eine Risikoabschätzung nur durch einen Rückgriff auf die Untersuchungen von 2006 möglich. Daher geht in Abschnitt 3.1.4 die Neuberechnung des LRF für die in den Kornhäusern gelagerten Sterilgutverpackungen den entsprechenden Berechnungen für Bad Rothenfelde und Oldenburg voraus.

Zur Berechnung des logarithmischen Reduktionsfaktors wurden exemplarisch für die Gesamtheit der in den Kornhäusern gelagerten Testeinheiten die Chargen aus Obernjesa herangezogen. Dies hat den Vorteil, dass die Testsysteme hier nicht nur im Aufzug deponiert waren, sondern auch außerhalb des Aufzugbetriebes gelagert wurden („stationäre Lagerung“). Eine vergleichende Darstellung dieser unterschiedlich platzierten Testeinheiten war somit direkt möglich. Des Weiteren bot sich dieser Standort an, da in diesem Kornhaus die Luftkeimzahlmessungen für die in Abschnitt 3.1.3 (Seite 43 - 44) folgenden Berechnungen erhoben wurden.

Aus der im Institut für Hygiene und Umweltmedizin durchgeführten und in Abschnitt 1.7 (Seite 18 - 19) dargestellten Quantifizierung durch die Verpackung eingedrungener und auf den Nährkarton sedimentierter Keime war es möglich, einen Mittelwert für die pro Verpackungseinheit vorhandene Verkeimung zu erheben. Die Ergebnisse hierzu sind in Abschnitt 3.1.4 auf Seite 46 zu finden. 


\section{Ergebnisse}

\subsection{Auswertung der in den Vorversuchen 2006 ermittelten Rohdaten - Lagerung der Testeinheiten in den}

Kornhäusern (Dransfeld - Scharzfeld - Obernjesa)

Die Temperaturschwankungen in den Kornhäusern (alle in Südniedersachsen gelegen) waren mit den äußeren Temperaturen praktisch identisch. Daher wurden für die sich anschließenden Berechnungen für alle drei Kornhäuser die täglichen Temperaturwerte in Göttingen als Bezugswerte herangezogen (URL www.wetterstation-goettingen.de).

Ähnlich wurde mit den Druckwerten verfahren. Für die erforderlichen Berechnungen dienten die täglichen witterungsbedingten atmosphärischen Luftdruckwerte in Göttingen (URL www.wetterstation-goettingen.de).

Als zusätzliche Komponente, um die Relevanz der alternierenden Druckschwankungen zu verdeutlichen, wurde die Möglichkeit genutzt, bestimmte Chargen in Aufzügen zu deponieren. Somit wirkten definierte Druckschwankungen in Höhe von maximal 3 hPa durch Aufzugsfahrten auf diese Testeinheiten. Durch die Anzahl der durchgeführten Aufzugsfahrten konnte der auf die Sterilgutverpackungssysteme einwirkende Druck ermittelt werden.

Vorherrschende Bedingungen:

- $\quad$ Extrem hohe Umgebungskeimlast von $8,3 * 10^{6} \mathrm{KbE} / \mathrm{m}^{3}$

- Tägliche Temperaturschwankungen entsprachen den Außentemperaturen

- Druckschwankungen entsprachen den täglichen wetterabhängigen atmosphärischen Druckwechseln (wirkten auf jede der fünf deponierten Chargen: Testgruppen Kornhaus Dransfeld, Kornhaus Obernjesa (mit und ohne Aufzugsbetrieb), Kornhaus Scharzfeld, Kontrollgruppe 1 (2006))

- Definierte atmosphärische Druckschwankungen durch Aufzugsfahrten (wirkten auf die in den Aufzügen deponierten Testgruppen: Kornhaus Dransfeld, Kornhaus Obernjesa, Kornhaus Scharzfeld ). 


\subsubsection{Determinante: atmosphärische Druckänderung}

\subsubsection{Einfluss von Luftdruckänderungen auf die gelagerten Testeinheiten in den Kornhäusern (Dransfeld, Obernjesa, Scharzfeld) und die Kontrollgruppe 1 (2006)}

Es führten lediglich Zunahmen der Luftdruckwerte zu einem Luftvolumenstrom ins Verpackungsinnere. Das Luftvolumen im Verpackungsinneren sank bei Erhöhung des Luftdruckes und durch die Poren auf der Papierseite der Papier-Folien-Verbundverpackung erfolgte ein ausgleichender Luftvolumeneinstrom in die Testeinheit.

Bei den Papier-Folien-Verbundverpackungen handelt es sich um ein gasdurchlässiges Verpackungssystem, es bliebt in seiner Form jedoch weitgehend starr.

Beim Absinken der Luftdruckwerte dehnte sich das in der Testeinheit enthaltene Luftvolumen aus und entwich durch die Poren der Papierseite in die umgebende Luft. Dieser Luftvolumenausstrom war für die folgenden Berechnungen jedoch nicht relevant, da hierdurch keine Keime ins Verpackungsinnere gelangten.

\subsubsection{Berechnung des druckabhängigen Volumenstroms durch die Papierseite der Testeinheit (Boyle- Mariotte- Gesetz)}

Um die Berechnungen durchführen zu können, wurden die täglich gemessenen witterungsabhängigen Luftdruckwerte von der Wetterstation Göttingen herangezogen (URL www.wetterstation-goettingen.de).

Anhand dieser Daten wurden die Druckdifferenzen zwischen den aufeinander folgenden Tagen der Lagerung berechnet. Hierbei waren für die folgenden Berechnungen nur jene Druckdifferenzen interessant, die einen Luftdruckanstieg bedeuten, denn nur die Zunahme des Luftdruckes führte zu einem Luftvolumeneinstrom ins Verpackungsinnere der Testeinheit.

Das approximative Volumen der Sterilgutverpackung der Testeinheit betrug ca. $240 \mathrm{ml}$. Dieses Volumen wurde in der Praxis durch die Gasphase Luft gebildet.

Die Temperatur wurde im Rahmen folgender Berechnungen als konstant betrachtet.

Die Berechnungen erfolgten gemäß den in Abschnitt 2.8.1 (Seite 33) dargestellten Berechnungen.

Eine tabellarische Auflistung der täglichen wetterabhängigen Luftdruckwerte sowie die Rechenschritte, welche zur Ermittlung des Luftvolumeneinstroms in das Verpackungsinnere einer Testeinheit (am jeweiligen Lagerungsort) führten, sind dem Anhang beigefügt (siehe Abschnitt 6.3.1.1, Seite 103 - 113). 
Tabelle 5:

Volumeneinstrom durch die wetterabhängigen atmosphärischen Druckänderungen während der entsprechenden Expositionszeit für die vier Kohorten aus den Kornhäusern und die Kontrollgruppe 1

\begin{tabular}{|c|c|c|}
\hline Testgruppe & $\begin{array}{c}\text { n } \\
\text { Anzahl der Testeinheiten }\end{array}$ & $\begin{array}{c}\qquad \Delta \mathbf{V}_{\text {wetterbhängig }} \\
\text { Volumeneinstrom pro Testeinheit (in ml) }\end{array}$ \\
\hline Dransfeld & 95 & 91,67 \\
\hline Obernjesa & 95 & 78,49 \\
\hline Scharzfeld & 95 & 78,49 \\
\hline Obernjesa* & 96 & 78,49 \\
\hline Kontrollgruppe 1 (2006) & 100 & 98,02 \\
\hline
\end{tabular}

* die in Obernjesa außerhalb des Aufzugbetriebes gelagerten Testeinheiten („stationäre Lagerung“)

Tabelle 6:

Volumeneinstrom durch die Druckänderungen bei einer Aufzugsfahrt

(Auch hier findet das Boyle- Mariotte- Gesetz Anwendung.)

\begin{tabular}{|c|c|c|}
\hline Testgruppe & $\begin{array}{c}\Delta \mathbf{p} \\
\text { Druckdifferenz pro } \\
\text { Aufzugfahrt } \\
\text { (in hPa) }\end{array}$ & $\begin{array}{c}\Delta \mathbf{V}_{\text {Aufzug }} \\
\text { Volumeneinstrom } \\
\text { pro Testeinheit } \\
\text { (in ml) }\end{array}$ \\
\hline " Dransfeld & 2,5 & 0,59 \\
\hline Obernjesa & 3,0 & 0,71 \\
\hline Scharzfeld & 2,5 & 0,59 \\
\hline
\end{tabular}

Jede Aufwärtsfahrt des Aufzuges förderte 0,71 ml (Obernjesa) beziehungsweise 0,59 ml (Dransfeld und Scharzfeld) Gasphase Luft aus der Sterilgutverpackung. Bei einer Abwärtsfahrt strömte das identische Volumen wieder in die Verpackung hinein.

Somit ergab eine Abwärtsfahrt ein Luftvolumeneinstrom von 0,71 ml (Obernjesa) beziehungsweise 0,59 ml (Dransfeld und Scharzfeld) in die Sterilgutverpackung hinein. Für die Berechnung wurde nur das Einstromvolumen herangezogen, da dieses Mikroorganismen in die Verpackung zu fördern vermag. 


\section{Tabelle 7:}

Betrachtung des resultierenden Gesamtvolumenstroms in das Innere der Verpackung durch die durch Aufzugsfahrten bedingten Druckschwankungen

\begin{tabular}{|c|c|c|c|c|}
\hline Testgruppe & $\begin{array}{c}\mathbf{N} \\
\text { Anzahl } \\
\text { Aufzugsfahrten }\end{array}$ & $\begin{array}{c}\text { n } \\
\text { Anzahl } \\
\text { Testeinheiten }\end{array}$ & $\begin{array}{c}\Delta \mathbf{\Delta} \mathbf{V}_{\text {Aufzug }} \\
\text { Volumeneinstrom } \\
\text { pro Testeinheit } \\
\text { (pro Aufzugsfahrt) } \\
\text { (in ml) }\end{array}$ & $\begin{array}{c}\Delta \mathbf{V}_{\text {Aufzug gesamt }} \\
\text { Volumeneinstrom } \\
\text { pro Testeinheit } \\
\text { bei } \mathrm{N} \\
\text { (in } \mathrm{ml} \text { ) }\end{array}$ \\
\hline Dransfeld & 190 & 95 & 0,59 & 112,1 \\
\hline Obernjesa & 167 & 95 & 0,71 & 118,57 \\
\hline Scharzfeld & 72 & 95 & 0,59 & 42,48 \\
\hline
\end{tabular}

Tabelle 8:

Resultierendes durch eine Verpackung strömendes Gesamtvolumen während der Expositionszeit (verursacht durch wetterabhängige und durch den Aufzugsbetrieb hervorgerufene Druckänderungen)

\begin{tabular}{|l|c|c|c|}
\hline \multicolumn{1}{|c|}{ Testgruppe } & $\begin{array}{c}\Delta \mathbf{V}_{\text {wetterabhänig }} \\
\text { Volumeneinstrom } \\
\text { durch wetterabhängige } \\
\text { Druckwechsel pro } \\
\text { Testeinheit }\end{array}$ & $\begin{array}{c}\Delta \mathbf{V}_{\text {Aufzug gesamt }} \\
\text { Volumeneinstrom } \\
\text { durch Aufzugsfahrten } \\
\text { pro Testeinheit } \\
\text { (in ml) }\end{array}$ & $\begin{array}{c}\mathbf{V}^{\circ} \text { gesamt } \\
\text { (in ml) } \\
\text { Solumeneinstrom durch die } \\
\text { Summe aller Druckänderungen } \\
\left(\Delta \mathbf{V}_{\text {wetterabhängig }}+\Delta \mathrm{V}_{\text {Aufzug gesamt }}\right) \\
\text { (in ml) }\end{array}$ \\
\hline Dransfeld & 91,67 & & 203,77 \\
Obernjesa & 78,49 & 112,1 & 197,06 \\
Obernjesa* & 78,49 & 42,48 & 120,97 \\
Kontrollgruppe 1 (2006) & 78,49 & -- & 78,49 \\
\hline
\end{tabular}

* die in Obernjesa außerhalb des Aufzugbetriebes gelagerten Testeinheiten („stationäre Lagerung“) 


\subsubsection{Determinante: Temperaturänderung}

\section{Darstellung der Temperaturschwankungen während des Expositionsintervalls der Testeinheiten in Dransfeld, Obernjesa und Scharzfeld}

Die in den Kornhäusern gelagerten Testeinheiten waren weitgehend den herrschenden Umgebungstemperaturen ausgesetzt.

Durch die Temperaturdaten der Wetterstation Göttingen (URL www.wetterstation-goettingen.de) war es möglich, den durch die Temperaturschwankungen verursachten Volumeneinstrom ins Verpackungsinnere der Testeinheiten zu berechnen. Hierbei kam es durch einen Temperaturabfall laut Charles`schem Gesetz bei konstantem atmosphärischen Luftdruck zu einem ins Verpackungsinnere gerichteten Luftstrom. Nähere Erläuterungen zu dieser Berechnungsgrundlage sind in Abschnitt 2.8.2 (Seite 34) dargestellt.

Die Kontrollgruppe 1 hingegen war lediglich sehr geringen täglichen Temperaturdifferenzen ausgesetzt, da im Institut für Hygiene und Umweltmedizin übliche Innentemperaturen von ca. $20{ }^{\circ} \mathrm{C}$ vorlagen. Hier wurde bei $20{ }^{\circ} \mathrm{C}$ eine tägliche Temperaturschwankung von $1^{\circ} \mathrm{C}$ angenommen.

\section{Volumenberechnung für die Kontrollgruppe 1:}

Die tägliche Temperaturdifferenz von $1{ }^{\circ} \mathrm{C}$ (bei einer Raumtemperatur von $20{ }^{\circ} \mathrm{C}$ ) führte laut Charles'schem Gesetz zu einem Volumeneinstrom von 0,86 ml pro Verpackung, pro Tag.

Da diese Testeinheiten 160 Tage lang eingelagert wurden, ist laut Charles'schem Gesetz mit einem Volumenstrom von 137,6 ml pro Verpackung zu rechnen.

\subsubsection{Volumenberechnung für die in Dransfeld, Obernjesa und Scharzfeld gelagerten Testeinheiten}

Um den durch Temperaturschwankungen verursachten Luftvolumeneinstrom für die in den Kornhäusern gelagerten Testeinheiten zu ermitteln, wurden Daten der Wetterstation Göttingen (URL www.wetterstationgoettingen.de) zu den sich anschließenden Berechnungen verwendet. Die entsprechende Datenreihe lieferte minimale und maximale Tagestemperaturwerte für die jeweilige Expositionsdauer.

Da lediglich der Temperaturabfall zu einem Luftvolumeneinstrom in das zu testende Verpackungssystem führte, wurden im Folgenden die minimalen und maximalen Tagestemperaturwerte (in Kelvin) in das Charles'sche Gesetz eingefügt und berechnet.

Eine tabellarische Auflistung der minimalen und maximalen Tagestemperaturen sowie ergänzende Rechenschritte, welche zur Ermittlung des Luftvolumeneinstroms ins Verpackungsinnere einer Testeinheit (am jeweiligen Lagerungsstandort) führten, sind dem Anhang zu entnehmen (siehe Abschnitt 6.3.1.2, Seite 114 121). 
Tabelle 9:

Darstellung des durch die Temperaturwechsel verursachten Volumeneinstroms in eine Testeinheit

\begin{tabular}{|c|c|c|}
\hline Testgruppe & $\mathbf{n}$ & $\begin{array}{c}\Delta \mathbf{V}_{\text {einzeln }} \\
\text { Anzahl Testeinheiten }\end{array}$ \\
\hline Dransfeld & 95 & 877,65 \\
\hline $\begin{array}{c}\text { Obernjesa } \\
\text { (mit und ohne } \\
\text { Aufzugsbetrieb) }\end{array}$ & 191 & 816,31 \\
\hline Scharzfeld & 95 & 816,31 \\
\hline
\end{tabular}

3.1.3 Kalkulation der mikrobiologischen Beanspruchung der Testeinheiten an den verschiedenen Standorten

Die Gesamtkeimzahl der Umgebungsluft in den Kornhäusern betrug hier 8,3*10 ${ }^{6} \mathrm{KbE} / \mathrm{m}^{3}$. Gemeinsam mit den in den Kornhäusern betriebenen Aufzugsfahrten stellte dies ein „Worst- Case- Szenario“ als eine extreme Belastungsprobe für die Testeinheiten dar.

Die Keimlast der Kontrollgruppe 1, welche in Göttingen deponiert wurde, wurde im Gegensatz hierzu mit lediglich $350 \mathrm{KbE} / \mathrm{m}^{3}$ angesetzt.

(Nähere Erläuterungen zur Erhebung der KbE- Werte an den verschiedenen Standorten sind in Abschnitt 2.7.1 auf Seite 30 - 31 zu finden.)

\section{A) durch wetterabhängige Druckschwankungen und den Aufzugsbetrieb verursachter Volumeneinstrom} und die damit einhergehende mikrobiologische Beanspruchung

\section{Dransfeld}

In diesem Kornhaus wurde von einer KbE- Zahl von 8,3*10 $10^{6} \mathrm{KbE} / \mathrm{m}^{3}$ Luft ausgegangen.

Hier gelangten durch die entstandenen Druckwechsel insgesamt 203,77 ml Luft in eine gelagerte Verpackung. Die mikrobiologische Beanspruchung pro Testeinheit betrug demnach 1691,29 KbE.

\section{Obernjesa}

Die in den Aufzugsbetrieb in Obernjesa eingebundenen Testeinheiten wurden durch diese Druckwechsel und die witterungsbedingten Druckalterationen einem Volumeneinstrom pro Verpackung von 197,06 ml ausgesetzt. Auch für diesen Betrieb wurde eine KbE- Zahl von 8,3*10 $\mathrm{KbE} / \mathrm{m}^{3} \mathrm{Luft}$ angenommen. Dies bedeutet für die Verpackung, dass in diesem Fall insgesamt 1635,60 KbE die gesamte mikrobiologische Beanspruchung darstellten.

Die 96 Testeinheiten der „stationären Lagerung“ aus Obernjesa wurden den Aufzugsfahrten nicht ausgesetzt. Hierfür ergab sich lediglich ein durch witterungsabhängige Druckdifferenzen hervorgerufener Volumeneinstrom pro Testeinheit von 78,49 ml Luft. Die mikrobiologische Beanspruchung dieser Testeinheiten betrug somit 651,47 KbE. 


\section{Scharzfeld}

Auch hier wurde eine $\mathrm{KbE}-\mathrm{Zahl}$ von 8,3*10 $\mathrm{KbE} / \mathrm{m}^{3}$ angenommen.

Bei den in Scharzfeld gelagerten 95 Testeinheiten kam es aufgrund der durch den Aufzugsbetrieb hervorgerufenen Druckwechsel und des schwankenden wetterabhängigen Luftdrucks zu einem Volumeneinstrom pro Testeinheit von 120,97 ml Luft. Dies bedeutet bei einer hohen KbE- Zahl von 8,3*10 $\mathrm{KbE} / \mathrm{m}^{3}$, dass 1004,05 KbE die gesamte mikrobiologische Beanspruchung für die einzelnen Verpackungen ausmachten.

\section{Kontrollgruppe 1 (2006)}

Durch witterungsbedingte atmosphärische Druckalterationen erfolgte in die im Institut gelagerten 100 Testeinheiten ein Volumeneinstrom pro Verpackung von 98,02 ml Luft.

Diese Proben waren jedoch keinen so ungünstigen Umgebungsbedingungen ausgesetzt wie es in den Kornhäusern der Fall war. Hier wurde eine $\mathrm{KbE}$ - Zahl von lediglich $350 \mathrm{KbE} / \mathrm{m}^{3}$ angenommen.

Die mikrobiologische Beanspruchung der Testeinheiten betrug hier nur 0,03 KbE.

\section{B) durch Temperaturschwankungen verursachter Volumeneinstrom und die damit einhergehende mikrobiologische Beanspruchung}

\section{Dransfeld}

In diesem Kornhaus wurde von einer KbE- Zahl von 8,3*10 $\mathrm{KbE} / \mathrm{m}^{3}$ Luft ausgegangen.

Durch die Temperaturschwankungen erfolgte ein Volumeneinstrom in eine von den hier deponierten 95 Testeinheiten von 877,65 ml Luft. Das stellte eine mikrobiologische Beanspruchung der Testeinheit von 7284,50 KbE dar.

\section{Obernjesa}

Auch für diesen Betrieb wurde eine KbE- Zahl von 8,3*10 $0^{6} \mathrm{KbE} / \mathrm{m}^{3}$ Luft angenommen.

Die in den Aufzügen gelagerten Testeinheiten und die Testeinheiten der „stationären Lagerung“ wurden hier zusammen betrachtet. In eine der in Obernjesa deponierten 191 Testeinheiten erfolgte durch die dort herrschenden Temperaturschwankungen ein Luftvolumeneinstrom von 816,31 ml Luft. Die mikrobiologische Beanspruchung betrug für eine hier deponierte Testeinheit somit 6775,37 KbE.

\section{Scharzfeld}

Auch für diesen Betrieb wurde eine KbE- Zahl von 8,3*10 $0^{6} \mathrm{KbE} / \mathrm{m}^{3}$ Luft angenommen.

Durch die alternierenden Temperaturschwankungen wurde bei den 95 in diesem Kornhaus gelagerten Testeinheiten ein Volumeneinstrom pro Verpackung von 816,31 ml Luft verursacht.

Daraus ergab sich für eine dort gelagerte Testeinheit eine mikrobiologische Beanspruchung von 6775,37 KbE.

\section{Kontrollgruppe 1 (2006)}

Für die Kontrollproben wurde eine KbE- Zahl von ledglich $350 \mathrm{KbE} / \mathrm{m}^{3}$ angenommen.

Die 100 Testeinheiten, die als Kontrollprobe in Göttingen eingelagert wurden, unterlagen nur einer geschätzten täglichen Temperaturdifferenz von $1^{\circ} \mathrm{C}$ unter Innenraumbedingungen bei $20^{\circ} \mathrm{C}$. Hierdurch ergab sich ein Volumeneinstrom pro Verpackung von 137,6 ml Luft in eine Testeinheit. Dies bedeutet eine mikrobiologische Beanspruchung einer hier gelagerten Testeinheit von 0,05 KbE. 
Daraus ergab sich für die mikrobiologische Beanspruchung in den Kornhäusern Folgendes:

Tabelle 10:

Anteil unsteriler Testeinheiten in den Kornhäusern

\begin{tabular}{|c|c|c|c|c|}
\hline Kornhaus & $\begin{array}{c}\text { Mikrobiologische } \\
\text { Beanspruchung } \\
\text { durch } \\
\text { Druckdifferenzen } \\
\text { pro Testeinheit } \\
\text { [KbE] }\end{array}$ & $\begin{array}{c}\text { Mikrobiologische } \\
\text { Beanspruchung } \\
\text { durch } \\
\text { Temperatur- } \\
\text { differenzen pro } \\
\text { Testeinheit } \\
\text { [KbE] } \\
\end{array}$ & $\begin{array}{c}\text { Gesamte } \\
\text { mikrobiologische } \\
\text { Beanspruchung } \\
\mathrm{N}_{0} \\
\\
{[\mathrm{KbE}]}\end{array}$ & Rekontaminationsrate \\
\hline Dransfeld & 1691,29 & 7284,50 & 8975,79 & 77,9 \\
\hline Obernjesa & 1635,60 & 6775,37 & 8410,97 & 84,2 \\
\hline Scharzfeld & 1004,05 & 6775,37 & 7779,42 & 69,5 \\
\hline Obernjesa* & 651,47 & 6775,37 & 7426,84 & 55,2 \\
\hline
\end{tabular}

*die in Obernjesa außerhalb des Aufzugbetriebes gelagerten Testeinheiten (, stationäre Lagerung“)

Abb. 7:

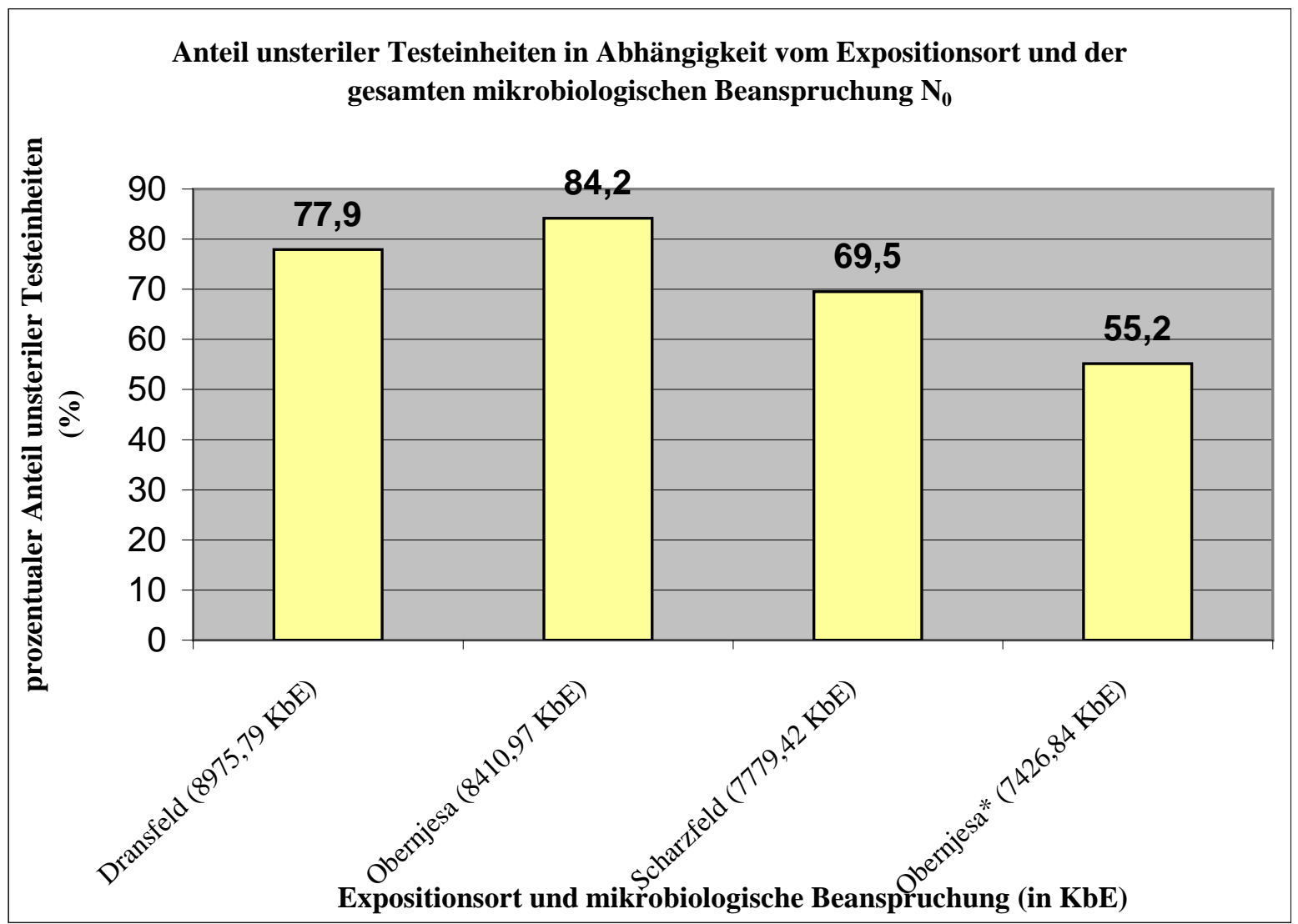

zu Abb. 7:

Anteil unsteriler Testeinheiten in Abhängigkeit vom Expositionsort und der gesamten mikrobiologischen Beanspruchung $\mathbf{N}_{0}$

Bei einer mikrobiologischen Beanspruchung $\mathrm{N}_{0}$ (Dransfeld: 8975,79 KbE/Testeinheit, Obernjesa: 8410,97 KbE/Testeinheit, Scharzfeld: 7779,42 KbE/Testeinheit, Obernjesa*: 7426,84 KbE) erwiesen sich zwischen 55,2 \% und 84,2 \% der Testeinheiten als unsteril. 


\subsubsection{Berechnung des LRF für die in Obernjesa gelagerten Sterilgutverpackungen}

Durch die in den Vorversuchen (2006) durchgeführte Quantifizierung durch die Verpackung eingedrungener und auf den Nährkarton sedimentierter Keime (siehe Seite 18 - 19), war es möglich, einen Mittelwert für die pro Verpackungseinheit nach Lagerung erfolgte Verkeimung zu erheben.

Für die in Obernjesa gelagerten Testeinheiten ohne Einfluss durch Aufzugsbetrieb fanden sich im Mittel nach der Expositionszeit 1,01 Pilze bzw. Keime auf dem Nährmedium jeder Verpackungseinheit.

Die Testeinheiten, welche zusätzlich den durch den Aufzugsbetrieb provozierten Druckschwankungen ausgesetzt waren, wiesen im Mittel bereits eine Verkeimung von 1,72 Keimen bzw. Pilzen pro Packungseinheit auf.

Durch diese Werte bot sich nun für die in Obernjesa auf unterschiedliche Arten gelagerten Chargen die Möglichkeit, den LRF für die Papierseite der Papier-Folien-Verbundverpackung zu errechnen.

Hierzu wurden noch einmal in Kürze die erforderlichen Daten aus den Tabellen 8 und 9 zusammengetragen:

\section{Tabelle 11:}

Obernjesa ohne Aufzugsbetrieb („stationäre Lagerung“) - Lufteinstrom pro Verpackung (in ml)

\begin{tabular}{|l|c|}
\hline Verschiedene Einflüsse des Luftdruckes & Lufteinstrom pro Verpackung (in ml) \\
\hline \hline - durch wetterabhängige Luftdruckwechsel verursachter & 78,49 \\
Volumeneinstrom & 816,31 \\
- durch Temperaturschwankungen verursachter Volumeneinstrom & 894,8 \\
$\rightarrow$ Summe Gesamtvolumeneinstrom: & \\
(durch wetterabhängige Druck- und durch Temperaturalterationen & \\
verursachter Gesamtvolumeneinstrom) & \\
\hline
\end{tabular}

Daraus ergab sich unter Berücksichtigung aller den Luftvolumenstrom beeinflussenden Faktoren (Temperatur/ Luftdruck) eine mikrobiologische Beanspruchung von 7426,84 KbE für eine Testeinheit der „stationären Lagerung“ in Obernjesa.

\section{Tabelle 12:}

\section{Obernjesa mit Aufzugsbetrieb - Lufteinstrom pro Verpackung (in ml)}

\begin{tabular}{|l|c|}
\hline Verschiedene Einflüsse des Luftdruckes & Lufteinstrom pro Verpackung (in ml) \\
\hline \hline - durch wetterabhängige Luftdruckwechsel verursachter & 78,49 \\
Volumeneinstrom & 118,57 \\
- durch Aufzugsfahrten provozierter Volumeneinstrom & 816,31 \\
- durch Temperaturschwankungen verursachter Volumeneinstrom & 1013,37 \\
$\rightarrow$ Summe Gesamtvolumeneinstrom: & \\
\hline (durch wetterabhängige Druckschwankungen, den Aufzugsbetrieb und & \\
die Temperaturschwankungen verursachter Gesamtvolumeneinstrom) & \\
\hline
\end{tabular}


Daraus ergab sich unter Berücksichtigung aller den Luftvolumenstrom beeinflussenden Faktoren (Temperatur/ wetterabhängiger Luftdruck/ Druckdifferenz durch Aufzugsfahrten) eine mikrobiologische Beanspruchung von 8410,97 KbE für eine der im Aufzugsbetrieb integrierten Testeinheiten in Obernjesa.

\section{$\underline{\text { KbE- Wert in Obernjesa }}$}

Für die Umgebungsluft der in den Kornhäusern gelagerten Testeinheiten wurde die für Obernjesa ermittelte Gesamtkeimzahl von $\underline{8,3^{*} 10^{\underline{6}}} \underline{\mathrm{KbE} / \mathrm{m}^{3}}$ Luft zugrundegelegt. Dieser KbE- Wert war für die Berechnung des LRF erforderlich.

\section{Berechnungen des LRF}

Der LRF für die Papier-Folien-Verbundverpackungen konnte bei bekannter mikrobiologischer Beanspruchung und nachweisbarer Rekontamination der in den Testeinheiten befindlichen Nährkartonscheibe mit folgender Formel berechnet werden:

$$
L R F=\log N_{0}-\log N_{1}
$$

Gleichzeitig konnte die Filtereffektivität der Verpackung durch folgende Gleichung berechnet werden:

Filterwirkung $(\%)=\left[1-N_{1} / N_{0}\right] * 100$

Für beide Gleichungen gilt:

$\mathrm{N}_{0}=$ durch den Volumenstrom und den Luftkeimgehalt auf des Verpackungsmaterial wirkende mikrobiologische Beanspruchung während der Expositionsdauer

(Für $\mathrm{N}_{0}$ werden hier die Werte der gesamten mikrobiologischen Beanspruchung N0 aus Tabelle 10 auf Seite 45 übernommen.)

$\mathrm{N}_{1}=$ Rekontamination der in der Verpackung enthaltenen Nährkartonscheibe nach Exposition, Inkubation und abschließender Quantifizierung der gewachsenen Kolonien

\section{A) für Obernjesa ohne Aufzugsbetrieb:}

$\rightarrow$ bei einem Gesamtvolumeneinstrom von 894,8 ml Luft ins Verpackungsinnere und einer Umgebungskeimlast von $8,3^{*} 10^{6} \mathrm{KbE} / \mathrm{m}^{3}$ bedeutet dies, dass pro Verpackung mit einer mikrobiologischen Beanspruchung von 7426,84 KbE zu rechnen war.

$$
\mathrm{LRF}=\log 7426,84-\log 1,01=\underline{3,87}
$$

Filterwirkung $(\%)=[1-1,01 / 7426,84] * 100=\underline{99,9864 \%}$ 


\section{B) für Obernjesa mit Aufzugsbetrieb:}

$\rightarrow$ bei einem Gesamtvolumeneinstrom von 1013,37 $\mathrm{ml}$ Luft ins Verpackungsinnere und einer Umgebungskeimlast von $8,3 * 10^{6} \mathrm{KbE} / \mathrm{m}^{3}$ bedeutet dies, dass pro Verpackung mit einer mikrobiologischen Beanspruchung von 8410,97 KbE zu rechnen war.

$$
\mathrm{LRF}=\log 8410,97-\log 1,72=\underline{3,69}
$$

Filterwirkung $(\%)=[1-1,72 / 8410,97] * 100=\underline{99,9796 \%}$ 


\subsection{Auswertung des Expositionsversuchs der Testeinheiten unter realitätsnahen Lagerungsbedingungen}

im medizinischen Betrieb

Im Anschluss an die Auswertung der in den Kornhäusern ermittelten Daten erfolgte als nächster Schritt eine Versuchsreihe unter realitätsnahen Bedingungen im Hinblick auf die Lagerung. Eine solch hohe Umgebungskeimlast wie sie in den Kornhäusern herrschte $\left(8,3 * 10^{6} \mathrm{KbE} / \mathrm{m}^{3}\right)$ war im klinischen Alltag natürlich nicht zu erwarten. Die Keimlast der Umgebungsluft war unter realitätsnahen Bedingungen wesentlich geringer einzuschätzen (siehe Abschnitt 2.7.1, Seite 30 - 31).

Zudem waren die Papier-Folien-Verbundverpackungen in Bad Rothenfelde keinen starken täglichen Temperaturschwankungen ausgesetzt, da hier klimatisierte Räumlichkeiten vorlagen.

Auf die in der Lagerhalle in Oldenburg deponierten Testeinheiten wirkten demgegenüber jedoch näherungsweise die herrschenden Außentemperaturen.

Lediglich die witterungsbedingten atmosphärischen Druckschwankungen an den verschiedenen Standorten hatten einen vergleichbaren Einfluss auf alle Verpackungssysteme (Kornhäuser, Oldenburg, Bad Rothenfelde). Hierzu wurden die erforderlichen Daten vom Deutschen Wetterdienst (Bundesministerium für Verkehr, Bau und Stadtentwicklung - Deutscher Wetterdienst (o.J.) URL www.dwd.de) und der Wetterstation Göttingen (URL www.wetterstation-goettingen.de) herangezogen.

\section{Die auf die Verpackungen wirkenden Umgebungsbedingungen nochmals kurz dargestellt:}

\section{Bad Rothenfelde:}

- Umgebungskeimlast von $150 \mathrm{KbE} / \mathrm{m}^{3}$ (siehe Abschnitt 2.7.1, Seite 30 -31)

- Annahme einer täglichen Temperaturschwankung von $1{ }^{\circ} \mathrm{C}$ (Raumtemperatur $20^{\circ} \mathrm{C}$, siehe Abschnitt 2.7.2, Seite 31)

- Druckschwankungen entsprachen den täglichen witterungsabhängigen atmosphärischen Druckwechseln (wirkten auf die gesamte deponierte Charge: die in Bad Rothenfelde gelagerten Testeinheiten wurden nach den zeitlich unterschiedlichen Rückführungsetappen in drei Gruppen aufgeteilt)

\section{Oldenburg:}

- Umgebungskeimlast von $350 \mathrm{KbE} / \mathrm{m}^{3}$ (siehe Abschnitt 2.7.1, Seite 30 - 31)

- Tägliche Temperaturschwankungen entsprachen Außentemperaturen (siehe Abschnitt 2.7.2, Seite 31 - 32)

- Druckschwankungen entsprachen den täglichen witterungsabhängigen atmosphärischen Druckwechseln (wirkten auf die gesamte deponierte Charge: die in Oldenburg gelagerten Testeinheiten wurden nach den entsprechenden Rückführungsetappen in drei Gruppen aufgeteilt)

\section{Kontrollgruppe 2 (2008):}

- Umgebungskeimlast von $350 \mathrm{KbE} / \mathrm{m}^{3}$ (siehe Abschnitt 2.7.1, Seite 30 - 31)

- Annahme einer täglichen Temperaturschwankung von $1^{\circ} \mathrm{C}$ (Raumtemperatur $20^{\circ} \mathrm{C}$, siehe Abschnitt 2.7.2, Seite 31)

- Druckschwankungen entsprachen den täglichen witterungsabhängigen atmosphärischen Druckwechseln 
Zwei Determinanten, die einen Lufteinstrom in die Sterilgutverpackung aufgrund der Gasdurchlässigkeit bedingen

Wie bereits in Abschnitt 2.8 (Seite 32 - 34) ausgeführt gelten auch hier bei Betrachtung der allgemeinen Gasgleichung ( $\mathrm{p}^{*} \mathrm{~V}=\mathrm{R} * \mathrm{~T}$ ) die Temperatur- sowie die Druckalterationen zu den entscheidenden Einflussgrößen im Hinblick auf daraus resultierende Volumenströme.

Da es sich auch hier um das gleiche flexible Verpackungssystem (flexibel im Sinn von gasdurchlässig, jedoch weitgehend formstabil) der Papier-Folien-Verbundverpackung handelte wie beim „Worst-Case-Szenario“, konnte eine Volumenänderung direkt ausgeglichen werden, indem ein entsprechender Volumenstrom ins Innere oder aber nach außen resultierte. Bei einem geschlossenen System ist dieser direkte Volumenausgleich nicht möglich.

\subsubsection{Determinante: atmosphärische Druckänderung}

\section{Atmosphärische Druckänderungen während des Expositionsintervalls der Testeinheiten der Sterilgutverpackungssysteme in Bad Rothenfelde und Oldenburg (und Kontrollgruppe 2) und resultierender Luftvolumeneinstrom}

Da eine Erhöhung der Luftdruckwerte das im entwickelten Testsystem enthaltene Luftvolumen komprimiert und die Papierseite der Testeinheit Poren enthält (offenes System), konnte zum direkten Volumenausgleich Luft durch diese Poren ins Verpackungsinnere strömen. Es resultierte ein Luftvolumeneinstrom und der Volumenausgleich bei diesem gasdurchlässigen aber weitgehend formstabilen Testsystem.

Die für anschließende Berechnungen des erfolgten Volumeneinstroms in die Testeinheiten erforderlichen täglichen wetterabhängigen atmosphärischen Luftdruckdaten wurden bei der Wetterstation Göttingen (URL www.wetterstation-goettingen.de) und dem Deutschen Wetterdienst (Bundesministerium für Verkehr, Bau und Stadtentwicklung - Deutscher Wetterdienst (o.J.) URL www.dwd.de) abgerufen.

Anhand dieser Daten wurden die Druckdifferenzen der während der Expositionsdauer aufeinander folgenden Tage berechnet. Hierfür waren nur jene Werte relevant, welche einen Luftdruckanstieg bedeuten.

Eine Übersicht der täglichen witterungsbedingten Luftdruckwerte zu den entsprechenden Expositionsintervallen und Lagerungsorten sowie Rechenschritte, die zur Ermittlung des Luftvolumeneinstroms in das Innere der Verpackung führten, sind im Anhang zu finden (Abschnitt 6.3.2.1 (Seite 122 - 134)). 


\subsubsection{Berechnung des druckabhängigen Volumenstroms durch die Papierseite der Testeinheit}

(Boyle- Mariotte- Gesetz)

Gemäß der Erläuterungen in Abschnitt 2.8.1 (Seite 33) fand auch hier zur Berechnung des druckabhängigen Volumenstroms das Boyle- Mariotte- Gesetz Anwendung.

\section{Tabelle 13:}

Volumenstrom hervorgerufen durch die atmosphärischen Druckänderungen im Rahmen der Kontrollgruppe 2 (2008)

\begin{tabular}{|l|c|c|}
\hline Rückführungsetappe & (n) & $\begin{array}{c}\Delta \mathbf{V}_{\text {Labor }} \\
\end{array}$ \\
& Anzahl Testeinheiten & $\begin{array}{c}\text { Volumeneinstrom durch atmosphärische } \\
\text { Druckschwankungen pro Testeinheit } \\
\text { (in ml) }\end{array}$ \\
\hline 31. 03. 2008-31.05. 2008 & 100 & 37,82 \\
\hline
\end{tabular}

\section{Tabelle 14:}

Volumeneinstrom hervorgerufen durch die atmosphärischen Druckänderungen während der Expositionszeit für eine Testeinheit der drei Kohorten aus Bad Rothenfelde

\begin{tabular}{|c|c|c|}
\hline Rückführungsetappen & $\begin{array}{l}\text { (n) } \\
\text { Anzahl Testeinheiten }\end{array}$ & $\begin{array}{c}\qquad \Delta \mathbf{V}_{\text {Bad Rothenfelde 1 }} \\
\text { Volumeneinstrom durch atmosphärische } \\
\text { Druckschwankungen pro Testeinheit } \\
\text { (in ml) }\end{array}$ \\
\hline 31. 03. $2008-10.06 .2008$ & 150 & 29,33 \\
\hline 31. 03. $2008-30.09 .2008$ & 150 & 73,98 \\
\hline 31. 03. $2008-08.12 .2008$ & 150 & 122,61 \\
\hline
\end{tabular}

\section{Tabelle 15:}

Volumeneinstrom hervorgerufen durch die atmosphärischen Druckänderungen während der Expositionszeit für eine Testeinheit der drei Kohorten aus Oldenburg

\begin{tabular}{|c|c|c|}
\hline Rückführungsetappen & $\begin{array}{c}\text { (n) } \\
\text { Anzahl Testeinheiten }\end{array}$ & $\begin{array}{c}\Delta \Delta \mathbf{V}_{\text {Oldenburg 1 }} \\
\text { Volumeneinstrom durch atmosphärische } \\
\text { Druckschwankungen pro Testeinheit } \\
\text { (in ml) }\end{array}$ \\
\hline 12. 07. $2008-22.09 .2008$ & 100 & 31,08 \\
\hline 12. 07. $2008-08.12 .2008$ & 100 & 83,16 \\
\hline 12. 07. $2008-30.03 .2008$ & 100 & 157,32 \\
\hline
\end{tabular}




\title{
3.2.2 Determinante: Temperaturänderung
}

\author{
Temperaturschwankungen während des Expositionsintervalls der Testeinheiten der \\ Sterilgutverpackungssysteme in Bad Rothenfelde, Oldenburg und Göttingen (Kontrollgruppe 2 (2008)) \\ und resultierender Volumeneinstrom
}

Anhand der Klimadaten des Deutschen Wetterdienstes (Bundesministerium für Verkehr, Bau und Stadtentwicklung - Deutscher Wetterdienst (o.J.) URL www.dwd.de) konnten die einzelnen Differenzen zwischen den maximalen und minimalen Temperaturen eines jeden Tages während der Expositionsdauer errechnet werden. Diese stellten einen Temperaturabfall dar, welcher laut Charles`schem Gesetz bei konstantem atmosphärischem Luftdruck eine Volumenabnahme des im Verpackungsinneren enthaltenen Luftvolumens mit sich brachte. Da es sich bei den Papier-Folien-Verbundverpackungen um ein offenes System handelt, wobei die Sterilgutverpackung gasdurchlässig ist aber formstabil bleibt, konnte durch einen ins Verpackungsinnere gerichteten Luftstrom ein direkter Volumenausgleich erfolgen. Durch den durch Temperaturwechsel hervorgerufenen Volumenverlust im Verpackungssystem erfolgte ein ausgleichender Volumeneinstrom.

\subsubsection{Berechnung der Volumenänderung in Abhängigkeit von der Temperaturänderung bei bekanntem} Ausgangsvolumen (Charles`sches Gesetz)

Gemäß der Schilderung in Abschnitt 2.8.2 (Seite 34) erfolgte auch hier die Berechnung der Volumenänderung aufgrund von Temperaturschwankungen.

Volumenberechnung für die in Göttingen gelagerte Kontrollgruppe 2 (2008)

Die in Göttingen deponierten Kontrollproben waren in den zwei Monaten Lagerungsdauer klimatisierten Verhältnissen ausgesetzt. Dies hat zur Folge, dass die auftretenden Temperaturdifferenzen sich in engen Grenzen hielten. Um den Volumenstrom in die Verpackung trotzdem rechnerisch erfassen zu können, wurde hier von einer täglichen Temperaturdifferenz von $1^{\circ} \mathrm{C}$ bei einer Raumtemperatur von $20{ }^{\circ} \mathrm{C}$ ausgegangen. Gemäß Charles`schem Gesetz ergab sich bei einer täglichen Temperaturdifferenz von $1^{\circ} \mathrm{C}$ für jede gelagerte Verpackung ein Volumeneinstrom der Gasphase Luft von 0,86 ml pro Tag (Lagerungsdauer insgesamt: 62 Tage).

Da diese Testeinheiten 62 Tage lang eingelagert wurden, ist laut Charles'schem Gesetz mit einem Volumenstrom von 53,32 ml pro Verpackung zu rechnen.

\section{Volumenberechnung für die in Bad Rothenfelde gelagerten Testeinheiten}

Aufgrund der in diesen Räumlichkeiten vorliegenden Innenraumbedingungen von $20^{\circ} \mathrm{C}$ wurde hier von einer täglichen Temperaturdifferenz von $1^{\circ} \mathrm{C}$ ausgegangen.

Daher ergab sich wie auch für die Kontrollgruppe 2 (2008) bei dieser Temperaturdifferenz täglich und pro Verpackung ein Luftvolumeneinstrom von 0,86 ml. 


\section{Tabelle 16:}

Volumeneinstrom durch Temperaturschwankungen für eine in Bad Rothenfelde gelagerte Testeinheit

\begin{tabular}{|l|c|c|}
\hline \multicolumn{1}{|c|}{$\begin{array}{c}\text { Rückführungsetappen } \\
\text { und Lagerungsdauer }\end{array}$} & \multicolumn{1}{c|}{$\begin{array}{c}\boldsymbol{\Delta} \mathbf{V}_{\text {Bad Rothenfelde 2 }} \\
\text { Volumeneinstrom durch } \\
\text { Anzahl Testeinheiten }\end{array}$} & $\begin{array}{c}\text { (n) } \\
\text { Temperaturschwankungen pro Testeinheit * } \\
\text { (in ml) }\end{array}$ \\
\hline $\begin{array}{l}\text { 31. 03. 2008-10. 06. 2008 } \\
\text { (72 Tage Lagerung) }\end{array}$ & 150 & 158,24 \\
\hline $\begin{array}{l}\text { 31. 03. 2008-30. 09. 2008 } \\
\text { (184 Tage Lagerung) }\end{array}$ & 150 & 217,58 \\
\hline 31. 03. 2008-08. 12. 2008 & 150 & \\
(253 Tage Lagerung) & & \\
\hline
\end{tabular}

*bei einem täglichen Luftvolumeneinstrom pro Testeinheit von 0,86 ml

\section{Volumenberechnung für die in Oldenburg gelagerten Testeinheiten}

Die in der Lagerhalle für Sterilgüter in Oldenburg deponierten Testeinheiten waren näherungsweise den dort herrschenden Außentemperaturen ausgesetzt. Es handelte sich nicht um klimatisierte Räumlichkeiten.

Auch hier fand das Charles'sche Gesetz zur Berechnung des temperaturabhängigen Luftvolumeneinstroms in die Testeinheit Anwendung. Die hierzu genutzte Datenreihe wurde dem Deutschen Wetterdienst (Bundesministerium für Verkehr, Bau und Stadtentwicklung - Deutscher Wetterdienst (o.J.) URL www.dwd.de) entnommen und ist im Anhang als Auflistung wiederzufinden. Ergänzt wurde diese Auflistung durch Rechenschritte, die zur Ermittlung des Luftvolumeneinstroms nötig waren (siehe Abschnitt 6.3.2.2, Seite 135 140).

\section{Tabelle 17:}

Volumeneinstrom durch Temperaturschwankungen für eine in Oldenburg gelagerte Testeinheit

\begin{tabular}{|l|c|c|}
\hline \multicolumn{1}{|c|}{$\begin{array}{c}\text { Rückführungsetappen } \\
\text { und Lagerungsdauer }\end{array}$} & \multicolumn{1}{c|}{$\begin{array}{c}\Delta \mathbf{V}_{\text {Oddenburg 2 }} \\
\text { Anzahl Testeinheiten }\end{array}$} & $\begin{array}{c}\text { Volumeneinstrom durch } \\
\text { Temperaturschwankungen pro Testeinheit } \\
\text { (in ml) }\end{array}$ \\
\hline $\begin{array}{l}\text { 12. 07. 2008- 22. 09. 2008 } \\
\text { (73 Tage Lagerung) }\end{array}$ & 100 & 606,53 \\
\hline $\begin{array}{l}\text { 12. 07. 2008-08. 12. 2008 } \\
\text { (150 Tage Lagerung) }\end{array}$ & 100 & 1029,8 \\
\hline $\begin{array}{l}\text { 12. 07. 2008-30. 03. 2009 } \\
\text { (262 Tage Lagerung) }\end{array}$ & 100 & 1581,12 \\
\hline
\end{tabular}


3.3 Kalkulation der mikrobiologischen Beanspruchung der Testeinheiten an den verschiedenen Standorten

In Bad Rothenfelde war mit einer geringen Keimbelastung der Umgebungsluft zu rechnen. Für diesen Lagerungsort unter Reinraumbedingungen wurde ein KbE- Wert von $150 \mathrm{KbE} / \mathrm{m}^{3}$ Luft angenommen.

Die Keimzahl der Umgebungsluft in der Lagerhalle in Oldenburg wurde mit dem Schätzwert $350 \mathrm{KbE} / \mathrm{m}^{3} \mathrm{den}$ Berechnungen zugrunde gelegt. Diese recht hohe KbE- Zahl war auf fehlende Luftreinigungsanlagen, eine belebte Umwelt und dadurch hervorgerufene turbulente Luftströmungen und eine sichtbare Oberflächenbeschmutzung der Lagerungsflächen zurückzuführen. Dieser Schätzwert von $350 \mathrm{KbE} / \mathrm{m}^{3} \mathrm{ergab}$ sich durch ( in Vorversuchen im Institut für Hygiene und Umweltmedizin durchgeführte) außenlufttechnische Messungen in Göttingen.

(Nähere Erläuterungen zu der Erhebung der KbE- Werte an den verschiedenen Standorten sind in Abschnitt 2.7.1, Seite 30 - $31 \mathrm{zu}$ finden.)

A) durch atmosphärische Druckschwankungen verursachter Volumeneinstrom und die damit einhergehende mikrobiologische Beanspruchung einer Testeinheit an den verschiedenen Standorten

\section{Bad Rothenfelde}

Aufgrund schwankender atmosphärischer Luftdrücke ergab sich für eine in Bad Rothenfelde gelagerte Testeinheit je nach Lagerungsdauer ein Luftvolumeneinstrom von 29,33 ml (1. Rückführungsetappe), 73,98 ml (2. Rückführungsetappe) oder 122,61 ml (3. Rückführungsetappe). Bei der angenommenen KbE- Zahl von 150 $\mathrm{KbE} / \mathrm{m}^{3}$ Luft betrug die mikrobiologische Beanspruchung einer Testeinheit 0,0044 KbE (1. Rückführungsetappe), 0,011 KbE (2. Rückführungsetappe) oder 0,018 KbE (3. Rückführungsetappe).

\section{Oldenburg}

An diesem Lagerungsort wurde die KbE- Zahl der Umgebungsluft auf $350 \mathrm{KbE} / \mathrm{m}^{3}$ Luft geschätzt.

In Oldenburg betrug der Luftvolumeneinstrom (hervorgerufen durch witterungsabhängige Druckschwankungen) in eine der hier gelagerten Testeinheiten 31,08 ml (1. Rückführungsetappe), 83,16 ml (2. Rückführungsetappe) oder 157,32 ml (3. Rückführungsetappe). Daraus resultierte eine mikrobiologische Beanspruchung für die einzelne Testeinheit von 0,011 KbE (1. Rückführungsetappe), 0,029 KbE (2. Rückführungsetappe) oder 0,055 KbE (3. Rückführungsetappe).

\section{Kontrollgruppe 2 (2008)}

Auch bei diesem Standort wurde eine KbE- Zahl der Umgebungsluft von $350 \mathrm{KbE} / \mathrm{m}^{3}$ Luft angenommen.

37,82 ml Luft strömten durch die erfolgten wetterahhängigen atmosphärischen Luftdruckwechsel ins Innere einer im Labor gelagerten Testeinheit. Bei einer KbE- Zahl von $350 \mathrm{KbE} / \mathrm{m}^{3}$ Luft lag eine mikrobiologische Beanspruchung einer im Labor gelagerten Verpackung von 0,013 KbE vor. 


\section{B) durch Temperaturschwankungen verursachter Volumeneinstrom und die damit einhergehende}

mikrobiologische Beanspruchung

\section{Bad Rothenfelde}

An diesem Lagerungsort wurde die KbE- Zahl der Umgebungsluft auf $150 \mathrm{KbE} / \mathrm{m}^{3}$ Luft geschätzt.

Der durch Temperaturschwankungen erfolgte Luftvolumeneinstrom in eine der 450 in Bad Rothenfelde gelagerten Testeinheiten betrug 61,92 ml (1. Rückführungsetappe),158,24 ml (2. Rückführungsetappe) oder 217,58 ml (3. Rückführungsetappe). Die Berechnung fand unter Berücksichtigung des Charles'schen Gesetzes statt. Der Grund für den im Vergleich zu den anderen Testkohorten geringen Volumenstrom kann in der konstanten Klimatisierung der Lagerstätte gesehen werden (tägliche Temperaturdifferenz von nur $1^{\circ} \mathrm{C}$ ).

Hieraus ergab sich für eine hier deponierte Testeinheit eine mikrobiologische Beanspruchung von 0,009 KbE (1. Rückführungsetappe), 0,024 KbE (2. Rückführungsetappe) oder 0,033 KbE (3. Rückführungsetappe).

\section{Oldenburg}

Bei diesem Standort wurde eine KbE- Zahl der Umgebungsluft von $350 \mathrm{KbE} / \mathrm{m}^{3} \mathrm{Luft}$ angenommen.

Der durch Temperaturschwankungen bedingte Luftvolumeneinstrom durch eine in Oldenburg gelagerte Testeinheit betrug 606,53 ml (1. Rückführungsetappe), 1029,8 ml (2. Rückführungsetappe) oder 1581,12 ml (3. Rückführungsetappe). Somit ließ sich für eine in Oldenburg gelagerte Testeinheit eine mikrobiologische Beanspruchung von 0,212 KbE (1. Rückführungsetappe), 0,36 KbE (2. Rückführungsetappe) oder 0,553 KbE (3. Rückführungsetappe) errechnen.

\section{Kontrollgruppe 2 (2008)}

An diesem Lagerungsort wurde die KbE- Zahl der Umgebungsluft auf $350 \mathrm{KbE} / \mathrm{m}^{3}$ Luft geschätzt.

Aufgrund der täglichen Temperaturdifferenz von $1^{\circ} \mathrm{C}$ ergab sich für eine der 100 im Labor gelagerten Testeinheiten ein Luftvolumeneinstrom in Höhe von 53,32 ml. Bei einem KbE- Wert von $350 \mathrm{KbE} / \mathrm{m}^{3}$ ergab sich daraus, dass die einzelne Verpackung einer mikrobiologischen Beanspruchung von 0,019 KbE ausgesetzt war. 
3.3.1 Vergleich der Rekontaminationsrate (\%) und der mikrobiologischen Beanspruchung der Testeinheiten unter den verschiedenen Expositionsbedingungen:

Kornhäuser (Dransfeld, Scharzfeld, Obernjesa) und medizinische Versorgungseinrichtungen ( Bad Rothenfelde, Oldenburg, Institut für Hygiene und Umweltmedizin Göttingen)

Erst im direkten Vergleich wird die Einflussgröße der Umgebungsbedingungen, denen die Testeinheiten ausgesetzt waren, deutlich. Anhand der folgenden Tabelle kann bereits ein direkter Zusammenhang zwischen der mikrobiologischen Beanspruchung und der hohen Rekontaminationsrate der in den Kornhäusern gelagerten Testeinheiten aufgezeigt werden. Um dies graphisch darstellen zu können, wurde zunächst die gesamte auf die an den unterschiedlichen Standorten deponierten Testeinheiten wirkende mikrobiologische Beanspruchung berechnet. Diese ergab sich aus der Summe der durch Druckschwankungen hervorgerufenen mikrobiologischen Beanspruchung und der durch Temperaturschwankungen bedingten mikrobiologischen Beanspruchung.

\section{Tabelle 18:}

Übersicht der gesamten mikrobiologischen Beanspruchung an den verschiedenen Lagerungsstandorten

\begin{tabular}{|c|c|c|c|c|}
\hline Testgruppe & $\begin{array}{l}\text { Mikrobiologische } \\
\text { Beanspruchung } \\
\text { durch } \\
\text { Druckdifferenzen } \\
\text { pro Testeinheit } \\
\text { [KbE] }\end{array}$ & $\begin{array}{c}\text { Mikrobiologische } \\
\text { Beanspruchung } \\
\text { durch } \\
\text { Temperatur- } \\
\text { differenzen pro } \\
\text { Testeinheit } \\
\text { [KbE] } \\
\end{array}$ & $\begin{array}{c}\text { Gesamte } \\
\text { mikrobiologische } \\
\text { Beanspruchung } \\
\mathrm{N}_{0} \\
{[\mathrm{KbE}]}\end{array}$ & $\begin{array}{c}\text { Rekontaminationsrate } \\
\text { [\%] }\end{array}$ \\
\hline Dransfeld & 1691,29 & 7284,50 & 8975,79 & 77,9 \\
\hline Obernjesa & 1635,60 & 6775,37 & 8410,97 & 84,2 \\
\hline Scharzfeld & 1004,05 & 6775,37 & 7779,42 & 69,5 \\
\hline Obernjesa* & 651,47 & 6775,37 & 7426,84 & 55,2 \\
\hline Kontrollgruppe1 (2006) & 0,03 & 0,05 & 0,08 & 0 \\
\hline $\begin{array}{l}\text { Bad Rothenfelde } \\
\text { (1.Rückführungsetappe) }\end{array}$ & 0,0044 & 0,009 & 0,0134 & 0 \\
\hline $\begin{array}{l}\text { Bad Rothenfelde } \\
\text { (2.Rückführungsetappe) }\end{array}$ & 0,011 & 0,024 & 0,035 & 0 \\
\hline $\begin{array}{l}\text { Bad Rothenfelde } \\
\text { (3.Rückführungsetappe) }\end{array}$ & 0,018 & 0,033 & 0,051 & 0 \\
\hline $\begin{array}{l}\text { Oldenburg } \\
\text { (1.Rückführungsetappe) }\end{array}$ & 0,011 & 0,212 & 0,223 & 0 \\
\hline $\begin{array}{l}\text { Oldenburg } \\
\text { (2.Rückführungsetappe) }\end{array}$ & 0,029 & 0,36 & 0,389 & 0 \\
\hline $\begin{array}{l}\text { Oldenburg } \\
\text { (3.Rückführungsetappe) }\end{array}$ & 0,055 & 0,553 & 0,608 & 0 \\
\hline Kontrollgruppe2 (2008) & 0,013 & 0,019 & 0,032 & 0 \\
\hline
\end{tabular}

*,stationäre Lagerung“ - ohne Einfluss des Aufzugsbetriebes 
Illustriert wird im nachfolgenden Diagramm die gesamte mikrobiologische Beanspruchung der an den verschiedenen Standorten deponierten Chargen. Überzeichnet wird diese Darstellung durch den prozentualen Anteil unsteriler Testeinheiten der unterschiedlichen Testgruppen.

Abb. 8:

Gesamte mikrobiologische Beanspruchung $\mathrm{N}_{0}$ und prozentualer Anteil unsteriler Testsysteme in Abhängigkeit vom Lagerungsort

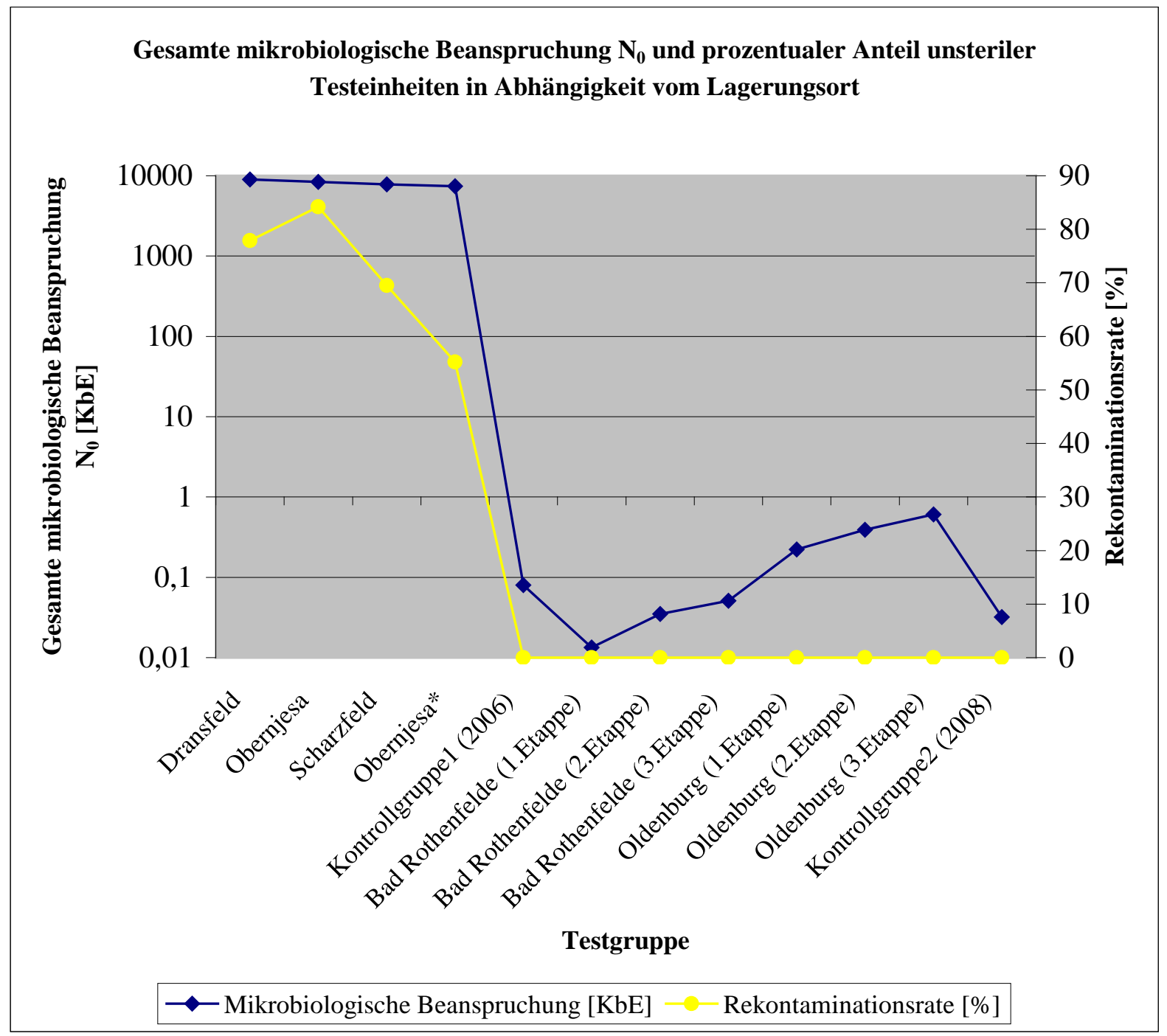

*stationäre Lagerung - ohne Einfluss des Aufzugsbetriebes

zu Abb. 8: Beziehung zwischen mikrobiologischer Beanspruchung und prozentualem Anteil unsteriler Testsysteme in Abhängigkeit vom Lagerungsort

In diesem Diagramm ist auf der $\mathrm{x}$ - Achse die jeweilige Testgruppe vermerkt, deren mikrobiologische Beanspruchung auf der linken Ordinate verzeichnet ist. Die rechte Ordinate ergänzt das Diagramm mit dem prozentualen Anteil unsteriler Testeinheiten an der entsprechenden Expositionsgruppe. Der Zusammenhang zwischen einer hohen mikrobiologischen Beanspruchung bei den Testgruppen Dransfeld, Obernjesa, Scharzfeld, Obernjesa* und dem deutlichen prozentualen Anteil unsteriler Testsysteme (bis zu 84,2\% unsteril) wird besonders deutlich. Sinkt die auf die Testsysteme wirkende mikrobiologische Beanspruchung (Kontrollgruppen, Bad Rothenfelde, Oldenburg) so erwiesen sich alle deponierten Testeinheiten als steril. 
Diese Darstellung legt nahe, dass der starke Rückgang von teilweise 84,2 \% unsteriler Testeinheiten (Obernjesa mit Aufzugbetrieb) in den Kornhäusern auf $0 \%$ unsteriler Testeinheiten sowohl in Oldenburg und Bad Rothenfelde als auch bei den Kontrollproben (im Institut für Hygiene und Umweltmedizin in Göttingen) durch die gewaltigen Unterschiede der mikrobiologischen Beanspruchung der verschiedenen Testgruppen zu begründen ist.

Die mikrobiologische Beanspruchung nahm mit einem großem Luftvolumeneinstrom ins Innere der PapierFolien-Verbundverpackung zu. Ein hoher Luftvolumenstrom wurde wiederum durch starke Temperaturschwankungen vor allem aber durch sich wiederholende Luftdruckwechsel bestimmt.

Die in den Kornhäusern hervorgerufenen Druckschwankungen (Aufzugsbetrieb) oder aber die gemessenen Temperaturdifferenzen (keine klimatisierten Verhältnisse) scheinen hier wichtige Determinanten eines erhöhten Rekontaminationsrisikos dargestellt zu haben. Zudem war der Keimgehalt der in die Verpackung strömenden Luft in den Kornhäusern $\left(8,3^{*} 10^{6} \mathrm{KbE} / \mathrm{m}^{3}\right)$ wesentlich höher als dies bei den Kontrollproben (350 KbE/m³), in Oldenburg (350 KbE/m³) oder sogar unter Reinraumbedingungen in Bad Rothenfelde $\left(150 \mathrm{KbE} / \mathrm{m}^{3}\right)$ der Fall war. 


\subsection{Berechnung der Rekontaminationswahrscheinlichkeit für die in Bad Rothenfelde und Oldenburg gelagerten Testeinheiten unter Berücksichtigung der anhand der Vorversuche 2006 (Kornhäuser) ermittelten Barrierewirkung}

Der LRF ist ein Wert, der für ein Verpackungsmaterial in Abhängigkeit von der auf die Verpackung wirkenden mikrobiologischen Beanspruchung und einer tatsächlichen Verkeimung des verpackten Gutes errechnet wird.

Dieser Faktor beschreibt somit die Reduktionsleistung des Verpackungsmaterials von der einwirkenden mikrobiologischen Beanspruchung auf die tatsächlich nachweisbare Verkeimung im Verpackungsinneren.

Die Filtereffektivität beschreibt wie viel von der mikrobiologischen Beanspruchung, die auf das Verpackungssystem wirkte, abgehalten werden konnte. Hierbei handelt es sich um eine prozentuale Angabe.

Aufgrund der relativ geringen mikrobiologischen Beanspruchung und des fehlenden Nachweises unsteriler Testeinheiten bei den Versuchen in Bad Rothenfelde und Oldenburg erscheint eine Ermittlung des LRF bzw. der Filtrationsleistung nicht sinnvoll.

Daher wird die Rekontaminationswahrscheinlichkeit für diese Standorte mit den ermittelten Reduktionsfaktoren aus den Vorversuchen in den Kornhäusern abgeschätzt.

$$
\mathrm{LRF}=\log \mathrm{N}_{0}-\log \mathrm{N}_{1}
$$

Hierzu wurde die Gleichung zur Berechnung des LRF folgendermaßen umgestellt:

$$
\log \mathrm{N}_{1}=\log \mathrm{N}_{0}-\mathrm{LRF}
$$

$\mathrm{N}_{1}$ ergibt somit die geschätzte Keimzahl (KbE) für die Rekontamination bei gegebener mikrobiologischer Beanspruchung $\mathrm{N}_{0}$ und bekanntem LRF. (Für $\mathrm{N}_{0}$ werden hier die Werte der gesamten mikrobiologischen Beanspruchung $\mathrm{N}_{0}$ aus Tabelle 18 auf Seite 56 übernommen.)

Beispielhaft wurde hier mit den Werten für die 3. Rückführungsetappe der in Bad Rothenfelde gelagerten Testeinheiten ein Rechenbeispiel erstellt:

\section{Rückführungsetappe Bad Rothenfelde}

- $\quad$ Anzahl der Testeinheiten: 150 Stück

- $\quad$ Mikrobiologische Beanspruchung: 0,051 KbE/Testeinheit (siehe Tabelle 18, Seite 56)

- $\quad$ Im Rahmen der Vorversuche (Obernjesa mit Aufzugsbetrieb) ermittelter LRF = 3,69 (siehe Seite 48)

$$
\begin{gathered}
\log \mathrm{N}_{1}=\log (0,051)-3,69=-4,98 \approx-5 \\
\rightarrow \mathrm{N}_{1}=0,00001
\end{gathered}
$$


Dies bedeutet, dass man bei Annahme eines LRF von 3,69 (laut Berechnungen für die Kornhäuser, siehe Seite 48) bei den in Bad Rothenfelde deponierten Testeinheiten pro Verpackung 0,00001 Keime gefunden hätte.

Das wiederum lässt die Schlussfolgerung zu, dass eine Kontamination einer Testeinheit am Standort Bad Rothenfelde auch rein theoretisch nicht zu erwarten war.

Denn bei einer berechneten durchschnittlichen Häufigkeit von 0,00001 Keimen pro Verpackung hätten demnach mindestens 100.000 Testeinheiten in Bad Rothenfelde deponiert werden müssen, um die statistische Wahrscheinlichkeit eines Keimnachweises erst möglich zu machen.

Da jedoch die Gesamtzahl der in Bad Rothenfelde gelagerten Testeinheiten lediglich 450 Stück betrug, war hier bei einer Barrierewirksamkeit entsprechend einem LRF von 3,69 auch nicht damit zu rechnen, einen Keimnachweis und somit den Nachweis für Unsterilität zu erbringen. Diese Erkenntnis deckt sich mit den in diesem Feldversuch erbrachten Resultaten.

Ähnlich verhielt es sich bei den in Oldenburg gelagerten Testeinheiten:

Beispielhaft wurde mit den Werten für die 3. Rückführungsetappe der in Oldenburg gelagerten Testeinheiten ein Rechenbeispiel erstellt:

\section{Rückführungsetappe Oldenburg}

- $\quad$ Anzahl der Testeinheiten: 100 Stück

- $\quad$ Mikrobiologische Beanspruchung: 0,608 KbE/Testeinheit (siehe Tabelle 18, Seite 56)

- $\quad$ Im Rahmen der Vorversuche (Obernjesa mit Aufzugsbetrieb) ermittelter LRF = 3,69 (siehe Seite 48)

$$
\begin{gathered}
\log \mathrm{N}_{1}=\log (0,608)-3,69=-3,906 \approx-4 \\
\rightarrow \mathrm{N}_{1}=0,0001
\end{gathered}
$$

Dies bedeutet, dass man bei einem LRF von 3,69 (laut Berechnungen für die Kornhäuser) bei den in Oldenburg gelagerten Testeinheiten pro Verpackungseinheit 0,0001 Keime gefunden hätte.

Das wiederum führt zu der Schlussfolgerung, dass eine Kontamination einer Testeinheit in der Lagerhalle Oldenburg auch rein theoretisch nicht zu erwarten war.

Denn bei einer berechneten durchschnittlichen Häufigkeit von 0,0001 Keimen pro Verpackung hätten demnach mindestens 10.000 Testeinheiten in der Lagerhalle deponiert werden müssen, um die statistische Wahrscheinlichkeit eines Keimnachweises auf einer der Nährkartonscheiben erst möglich zu machen.

Somit war hier bei einer Barrierewirksamkeit entsprechend einem LRF von 3,69 nicht davon auszugehen, dass bei einer Gesamtzahl von 300 Testeinheiten in Oldenburg nach Transport, Lagerung und Aktivierung eine unsterile Testeinheit zu finden ist.

Diese theoretische Annahme deckt sich mit den in diesem Feldversuch erbrachten Ergebnissen. 


\subsection{Aufrechterhaltung der Sterilität unter Berücksichtigung des Sterilitätssicherheitswertes - sterility assurance level (SAL)}

Wie auf Seite 36 (Abschnitt 2.9) dargestellt, wird Sterilität quantitativ über den SAL (sterility assurance level, Sterilitätssicherheitswert) $=10^{-6}$ definiert.

Für die logarithmische Gleichung ergibt sich:

$$
\log \mathrm{N}_{0}-\mathrm{LRF} \leq-6
$$

Ergibt sich demnach unter Berücksichtigung tatsächlicher Messdaten ein Wert von > -6, dann wurde keine Sterilität im Sinne des SAL während der Lagerung aufrecht erhalten.

3. Rückführungsetappe Bad Rothenfelde

- $\quad \mathrm{N}_{0}=0,051 \mathrm{KbE} /$ Testeinheit (siehe Tabelle 18, Seite 56)

- $\quad$ Im Rahmen der Vorversuche (Obernjesa mit Aufzugsbetrieb) ermittelter LRF = 3,69 (siehe Seite 48)

$$
\log 0,051-3,69=-4,98
$$

$\rightarrow$ Demnach wurde die Sterilität im Sinne des SAL unter den Expositionsbedingungen in Bad Rothenfelde nicht aufrecht erhalten.

\section{Rückführungsetappe Oldenburg}

- $\quad \mathrm{N}_{0}=0,608 \mathrm{KbE} /$ Testeinheit (siehe Tabelle 18, Seite 56)

- $\quad$ Im Rahmen der Vorversuche (Obernjesa mit Aufzugsbetrieb) ermittelter LRF = 3,69 (siehe Seite 48)

$$
\log 0,608-3,69=-3,91
$$

$\rightarrow$ Demnach wurde die Sterilität auch unter den Expositionsbedingungen in Oldenburg nicht aufrecht erhalten.

Durch entsprechende Umstellung der Gleichung $\quad \log \mathrm{N}_{0}-\mathrm{LRF} \leq-6 \quad$ nach $\quad$ LRF erforderlich $=\log \mathrm{N}_{0}+6$ wird die erforderliche Barrierewirksamkeit (angegeben als LRF erforderlich ) bei gegebener mikrobiologischer Beanspruchung ermittelt.

Diese Berechnung des erforderlichen LRF unter Berücksichtigung des Sterilitätssicherheitswertes (SAL) wurde anhand der 3. Rückführungsetappe an den Standorten Bad Rothenfelde und Oldenburg beispielhaft durchgeführt:

\section{Rückführungsetappe Bad Rothenfelde}

$\mathrm{N}_{0}=0,051 \mathrm{KbE} /$ Testeinheit (siehe Tabelle 18, Seite 56)

$$
\mathrm{LRF}_{\text {erforderlich }}=\log 0,051+6=4,71
$$




\section{Rückführungsetappe Oldenburg}

$\mathrm{N}_{0}=0,608 \mathrm{KbE} /$ Testeinheit (siehe Tabelle 18, Seite 56)

$$
\mathrm{LRF}_{\text {erforderlich }}=\log 0,608+6=5,78
$$

Dies bedeutet, dass die Verpackung bei den in Bad Rothenfelde herrschenden Lagerungsbedingungen einen

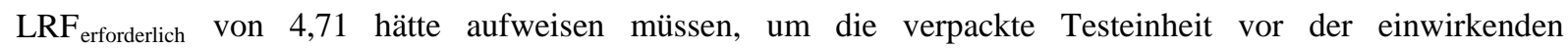
mikrobiologischen Beanspruchung zu schützen und die Sterilität des Verpackungsinneren aufrecht zu erhalten.

Aufgrund der für die Lagerung von Sterilgut ungünstigeren Bedingungen in der Lagerhalle Oldenburg hätte das hier eingesetzte Verpackungsmaterial einen LRF erforderlich von 5,78 aufweisen müssen, um die verpackte Testeinheit vor der einwirkenden mikrobiologischen Beanspruchung zu bewahren und das Verpackungsinnere steril zu halten. 


\section{Diskussion}

\subsection{Wahrung der Sterilität verpackter Medizinprodukte}

Im Anschluss an die ermittelten Werte und ausgeführten Rechnungen ist es unumgänglich, sich noch einmal mit dem Begriff der „Sterilität“ auseinander zu setzen. Nach der in der Einleitung bereits aufgeführten Begriffsdefinition der Sterilität von Medizinprodukten wird ein „von lebensfähigen Mikroorganismen freier Zustand“ (DIN EN 556-1 (2001) S.5) gefordert.

Eine Keimzahlreduktion muss in der Weise erfolgen, dass nach dem Sterilisationsprozess die Wahrscheinlichkeit für 1 unsteriles Produkt höchstens 1:1.000.000 beträgt.

„Während der Sterilisationsprozess durch die Prozesssteuerung zuverlässig erreicht werden kann, ist die Aufrechterhaltung der Sterilität bis zum Zeitpunkt der Anwendung des Medizinproduktes ein - wie in DIN EN ISO 11607-1 betont - schwieriges und kritisches Unterfangen, weil Sterilbarrieresysteme aufgrund ihrer unterschiedlichen funktionalen Komponenten sowie auch aufgrund der Notwendigkeit der Luft- und Dampfdurchlässigkeit oder aufgrund von Defekten durch Alterung und Verschleiß zahlreiche Risiken der Kontamination bergen“ (Dunkelberg und Schmelz 2008a, S.132).

Die erbrachten Berechnungen und theoretischen Annahmen im Rahmen dieser Arbeit zeigen die Problematik der Wahrung der Sterilität durch Sterilgutverpackungssysteme. Der Fokus der Beobachtungen wurde auf das System der Papier-Folien-Verbundverpackung gelegt.

Die Betrachtungen der atmosphärischen Druckschwankungen und Temperaturdifferenzen während der jeweiligen Lagerungszeiten an verschiedenen Standorten und die daraus resultierenden Volumenströme ins Verpackungsinnere ließen theoretische Berechnungen zur Kontaminationswahrscheinlichkeit des verpackten Gutes zu.

Es ist anzumerken, dass der Volumenausstrom aus der Verpackung und auch die durch Diffusionsereignisse eindringenden Mikroorganismen unberücksichtigt blieben.

Diese Berechnungen weisen nach, dass die Wahrung der Sterilität durch Papier-Folien-Verbundverpackungen mit einem geforderten Sterilitätssicherheitsniveau von 1:1.000.000 auch bei üblichen Umgebungsbedingungen und Lagerungsdauer nicht sicher ist, während unter extrem hoher aerogener Luftkeimzahl eine hohe Rate unsteriler Produkte zu erwarten ist.

Am Beispiel der in Obernjesa (Vorversuche 2006) gelagerten Testeinheiten konnte dies besonders eindrucksvoll aufgezeigt werden. Hier wurden für eine Zeitspanne von ca. 3 1⁄2 Monaten eine Stückzahl von 95 Testsystemen im Aufzugsbetrieb integriert gelagert. Hiervon befanden sich nach Rückführung 80 Stück in einem unsterilen Zustand. Dies betrug einen Anteil an unsterilen Testeinheiten von 84,2 \%. Nach Aktivierung des mikrobiologischen Teils der Testeinheit und Anzüchtung eingedrungener und sedimentierter Keime konnte in diesen Fällen also eine Kontamination des verpackten Sterilgutes nachgewiesen werden.

Daher wiesen diese Medizinprodukte keine Sterilität entsprechend der normativen Definition (DIN EN 556-1 (2001)) auf. 
„Zurückgehend auf das Medizinproduktgesetz und die darin geforderte ,Sterilhaltung bis zur Anwendung` gehören neben Verpackungssystem und Validierung auch Lagerdauer und -umstand zum fehlerfreien Verpackungsprozess“ ( Wolf C 2003, S. 254).

Dies bedeutet für die Zukunft, vor dem Hintergrund der Tatsache, dass immer häufiger externe Unternehmen mit der Sterilisation beauftragt werden und somit mehr Transportvorgänge ablaufen, dass eine Klassifizierung der Sterilgüter „über eine aktionsbezogene Abhängigkeit der Lagerdauer ( ,Event-Related`)“ (Wolf C 2003, S. 254) notwendig wird.

\section{2 „Event- related” und „Time- related”: ereignisbezogener und zeitbezogener Sterilitätsverlust}

Hier ist die Differenzierung der Begriffe „Event- related“ und „Time- related“ erforderlich.

„Time- related“ definiert hierbei die mögliche Lagerungsdauer allein anhand des Faktors Zeit, während der Begriff „Event- related“ vorherrschende Umgebungsbedingungen wie Handhabung, Transportmechanismen und Umgebungskeimlast berücksichtigt. „Event- related“ bedeutet, dass es unabhängig von der Lagerungsdauer zu einem Ereignis kommt, welches zu einer Kontamination des Sterilgutes führt. Hierzu wird in den meisten Studien auch die Beschädigung der Verpackung im Rahmen der Handhabung gezählt.

Diese Behauptung wird ebenfalls von Schwartz und Davis unterstützt. „The findings of this study support the proposal that contamination is event related, not time related; that is, sterile instrument packs remain sterile for at least 1 year unless a specific event causes contamination“ (Schwartz und Davis 1990, S.299).

Auch Klapes et al. 1987b konnten in ihren Untersuchungen im Rahmen der Studie „Effect of Long- term Storage on Sterile Status of Devices in Surgical Packs” (Klapes et al. 1987b, S.289) keinen Trend einer zunehmenden Kontaminationswahrscheinlichkeit während der Lagerungszeit für eine der untersuchten Verpackungstypen beobachten.

Ähnlich fällt auch nachstehende Schlussfolgerung von Webster et al. 2003 aus : „, We believe that event-related outdating is safe and cost-effective and should replace time-related expiry dating” (Webster et al. 2003, S. 623).

Nach dieser Betrachtungsweise stellt sich hier jedoch die Frage, ab wann der Begriff „Event- related“ als zutreffend bezeichnet werden kann. Zählt hierzu lediglich die fehlerhafte Handhabung des Sterilgutes, die Beschädigung der Verpackung zum Beispiel durch versehentliches Durchstoßen oder Ähnliches, oder sind hierunter bereits wechselnde atmosphärische Druckschwankungen oder auch Temperaturwechsel zu verstehen?! Aufgrund dieser „Grauzone“ in der Begriffsdefinition ist eine individuelle Einschätzung und Klassifizierung des Sterilgutes im Hinblick auf die jeweilige Lagerungsdauer, Transportprozesse und vorherrschende Umgebungsbedingungen nötig.

Zudem ist es fraglich, wie ein rein ereignisbezogenes Geschehen an den Sterilgutverpackungen zu detektieren ist. Die Untersuchung einer jeden Verpackung auf kleine Durchstiche oder Undichtigkeiten wäre wesentlich zeitintensiver wie auch unzuverlässiger als eine erneute Verpackung und Sterilisation des Medizinproduktes.

Widmer et al. 1992 entwickelte mit seiner Studie „A new standard for sterility testing for autoclaved surgical trays“ die erste Testreihe mit einem standardisierten Programm bzw. einer Abfolge von Ereignissen (,,events“), denen das sterilisierte und verpackte Gut ausgesetzt war. Im Rahmen dieser Untersuchungen erfolgte die Einhaltung eines detaillierten Protokolls an „events“, welchen das Sterilgut ausgesetzt wurde. Tatsächlich zeigte er mit einer ermittelten odds ratio von 3,5 , dass diese „events“ die Kontaminationswahrscheinlichkeit 
beeinflussen. „Surgical packs that were exposed to the events had a 3,5 greater probability of becoming contaminated than the controls“ (Widmer et al. 1992, S. 258).

Anzumerken ist ferner, dass die Testeinheiten in Oldenburg und Bad Rothenfelde mit ca. 8 Monaten wesentlich länger gelagert wurden als die in den Kornhäusern deponierten Testeinheiten (max. 4 Monate). Dies lässt darauf schließen, dass das Rekontaminationsrisiko nicht in erster Linie von der Lagerungsdauer der Sterilgutverpackungen abhing, sondern vielmehr von der mikrobiologischen Beanspruchung, der das Verpackungssystem ausgesetzt war. Betrachtet man die hohe Rekontamination der Testgruppen Dransfeld, Obernjesa, Obernjesa* und Scharzfeld und bezeichnet man die beeinflussenden Faktoren wie Temperatur, atmosphärische Druckverhältnisse und KbE- Zahl der Umgebungsluft als Ereignisse, die auf das verpackte Medizinprodukt wirkten, so kann hier von einer ereignisbezogenen Wiederverkeimung gesprochen werden.

Das Produkt bleibt solange steril wie Ereignisse vorliegen, die unter Berücksichtigung des SAL (sterility assurance level) mit der Barriereeigenschaft der Verpackung vereinbar sind.

\subsection{Vorteile der im Verpackungssystem integrierten Testeinheiten und Grenzen des Sterilitätstests}

Um die Vorteile der entwickelten Testeinheiten deutlich darstellen zu können, ist eine kurze Erläuterung des gewöhnlichen Sterilitätstests nach der Lagerung hilfreich. Gängige Sterilitätstests werden unter möglichst aseptischen Bedingungen durchgeführt. Hierzu gehören aseptische Bekleidung des Laborpersonals, aseptische Räumlichkeiten (eventuell Schleusensysteme), aseptische Instrumente und die durch entsprechende Reinluftanlagen („Sterilbänke“) maximal keimreduzierte Luft.

Die Grenzen eines solchen Sterilitätstests sind zum einen durch die zu untersuchende Stückzahl gegeben. Dies führt zu einer statistischen Unsicherheit in Abhängigkeit von der Anzahl überprüfter Sterilgüter. Das bedeutet also für eine untersuchte Stückzahl von z.B. 1000 Sterilgütern, dass der Begriff steril im Sinne des SAL hier bereits keine Anwendung mehr finden darf. Die Anforderung an Sterilität ist nämlich Keimfreiheit im Sinne der Wahrscheinlichkeit von max. $10^{-6}$ Keimen pro Produkt. In dem geschilderten Beispiel von einer überprüften Anzahl von 1000 Medizinprodukten wird die normativ geforderte Wahrscheinlichkeit nicht erreicht.

Des Weiteren können methodische Fehler angeführt werden.

Zum einen kann durch eingeschleppte Keime durch das Personal oder aber durch einströmende Mikroorganismen beim Öffnen der Verpackung eine Kontamination erfolgen. Hierdurch können falsch positive Ergebnisse hervorgerufen werden.

Aufgrund dieser Tatsache wird bei gängigen Sterilitätstests mit einem labortechnisch bedingtem und daher unvermeidbarem Fehleranteil gerechnet. Dies wiederum birgt die Gefahr eventuell während der Lagerungsdauer in die Verpackung eingedrungene Mikroorganismen aufgrund entsprechender Kontrollwerte als labortechnischen Fehler zu beurteilen. Die untersuchte Rekontaminationsproblematik kann daher nicht angemessen reproduziert werden, da die Problematik des methodischen Fehlers überwiegt bzw. dieser Fehler eine gegebene Kontamination nicht eindeutig erkennbar macht.

Dieser methodische Fehler ist in einer Vielzahl wissenschaftlicher Arbeiten zu finden (Klapes et al. 1987a; Klapes et al. 1987b; Cooper 1982; Kelsey 1972; Odlaug et al. 1984). In diesen Studien wird eine Kontamination der untersuchten Proben von $0,1 \%$ bis sogar 5,0\% beschrieben, welche alle samt als vernachlässigbar gering gewertet werden. Die geringe Anzahl kontaminierter Produkte wird hier auf Kontaminationen beim Öffnen und 
bei der Handhabung der Prüfkörper zurückgeführt. Diese unterschiedlichen Ergebnisse im Hinblick auf den prozentualen Anteil an kontaminierten Proben bei den verschiedenen Sterilitätstests werden u.a. durch den Schwierigkeitsgrad des jeweiligen Tests und der zu untersuchenden Gegenstände erklärt (Kelsey 1972).

Schwartz und Davis 1990 sehen in ihrer Publikation „Safe storage times for sterile dental packs“ eine Vernachlässigung von 1-2\% unsteriler Verpackungen als angemessen. Sie schildern die Ursache der Kontamination wie folgt: „These may have been due to failure of the packing materials, but more likely the contamination was introduced during the culturing process. It is well documented in industry and hospital sterilization services that low levels of contamination occur because of failures in aseptic transfer " (Schwartz und Davis 1990, S.299).

Dieser gerade beschriebene, häufig wiederzufindende, tolerierte Fehler in einer Größenordnung von 0,1\% - 5,0\% überschreitet jedoch bei weitem die Toleranzgrenze, welche die Begriffsdefinition der Sterilität mit sich bringt.

Es kann bereits bei einer Kontamination größer als 0,000001\% der Medizinprodukte nicht mehr von einer Wahrung der Sterilität gesprochen werden. (Sterilität wäre dann gegeben, wenn unter 0,000001\% der Medizinprodukte unsteril wären.)

Wie soll es daher jedoch möglich sein, die Verletzung der Sterilität nach der Lagerung nachzuweisen, wenn ein methodischer Fehler von bis zu 5\% toleriert wird ?!

Ein Wert, der den Begriff der Sterilität um das 5.000.000- fache verfehlt.

\section{Möglichkeit des Keimnachweises bei geschlossenem Verpackungssystem}

Der wohl entscheidende Vorteil der entwickelten Testeinheit ist, dass die Anzüchtung eingedrungener Keime ohne eine Öffnung des Verpackungssystems durchgeführt werden kann. Dadurch kann der Anteil falsch positiver Ergebnisse durch eventuelle labortechnische Fehler (falsch positive Ergebnisse) weitgehend unterbunden werden. Außerdem wird der oben aufgeführte methodische Fehler ausgeklammert und somit kann jede nachweisbare Kontamination auch als selbige gewertet werden. Das bedeutet: Kultivierte Kolonien können praktisch nicht durch Querkontaminationen im Zuge der Handhabung bedingt sein, sondern werden ausschließlich durch eine Rekontamination des Sterilgutes durch die Verpackung hindurch verursacht. Sie können demnach direkt für die Bewertung der Barrierewirkung genutzt werden.

Zudem wird bei diesem System der Alterungsprozess der Verpackung gleichzeitig mit berücksichtigt. Aseptische Umgebungsbedingungen für die Auswertung sind bei diesem neuartigen Testsystem nicht mehr erforderlich, da das Sterilgut sicher verschlossen im Inneren der Verpackung verbleibt. Somit kann auf aseptische Kleidung, aseptische Räumlichkeiten und besondere Belüftungsmaßnahmen verzichtet werden. Neben einer erheblichen Aufwandsminderung, führt dies ebenfalls zu einer nicht zu vernachlässigenden finanziellen Ersparnis.

Weiteres kennzeichnendes Merkmal der entwickelten Testeinheit stellt die Validierbarkeit dieses Systems dar, wodurch im täglichen klinischen Ablauf eine Anwendung im Sinne einer Qualitätssicherung durchführbar ist.

Die Überprüfung der Barrierefunktion kann nach erfolgter Lagerungszeit und direkt vor der Sterilgutanwendung stattfinden. Dadurch wird die Wahrscheinlichkeit erhöht, dass sich auch das Sterilgut unmittelbar vor der Anwendung in einem mikrobiologisch einwandfreien Zustand befindet. Eine mitgeführte Testeinheit kann als Stichprobe ausgewertet werden. Je nach der Stückzahl einer Charge beigefügter Testeinheiten steigt die Aussagekraft des Befundes der Testeinheiten im Hinblick auf die Sterilität der Medizinprodukte dieser Charge. 


\subsection{Verbesserungsentwurf der entwickelten Testeinheit}

Der enorme Produktionsaufwand der im Rahmen dieser Untersuchungen Anwendung findenden Testeinheit in Handarbeit stellt für den Gebrauch im klinischen Alltag jedoch eine zu zeitintensive und daher kostspielige Prüfmethodik dar. Daher muss hier im Sinne einer möglichen Serienfertigung eine Vereinfachung der Produktion angestrebt werden.

Zudem muss die Integrität der Papier-Folien-Verbundverpackung bewahrt werden, indem zum Beispiel der mikrobiologische Teil der Testeinheit sowie der Aktivierungsteil in einer entsprechenden Kunststofffassung (Tiefzieh- Blister) gegen Verrutschen gesichert werden (vgl. Abb. 9) und so eine Beschädigung der Papierseite durch in der Verpackung enthaltene Elemente verhindert wird.

Abb. 9:
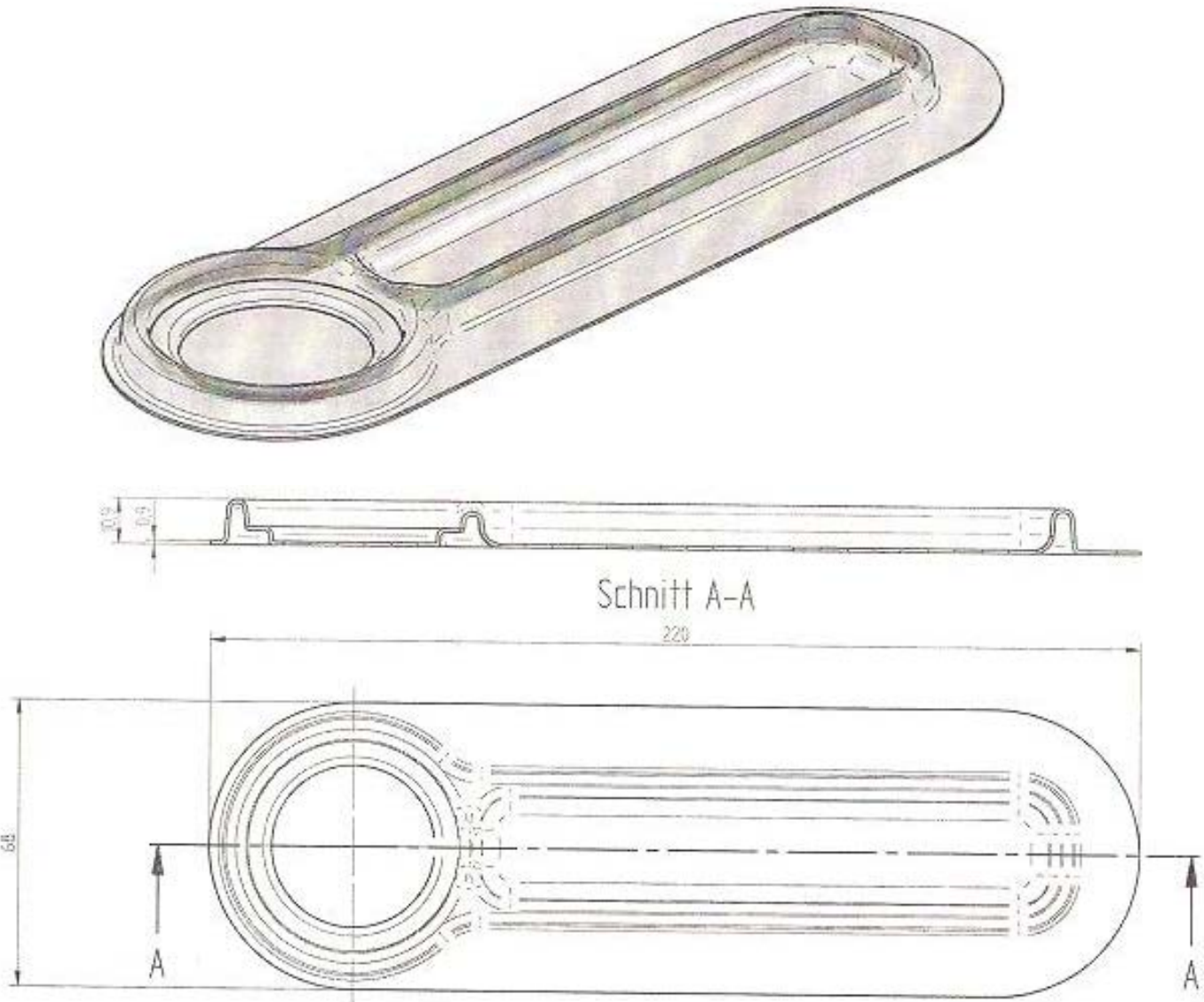

\section{Mögliche serienfertigungsfähige Halterung für die beiden Komponenten der Testeinheit}

Medipack 2006

(im Rahmen der Entwicklung der Testeinheit in Auftrag gegeben)

Entwurf: Medipack, CH- Schaffhausen, 2006 


\section{Flächenverhältnis}

Ein weiterer die Rekontaminationswahrscheinlichkeit der Testeinheit reduzierender Faktor stellt die Größe der Nährkartonscheibe im Verhältnis zur Papieroberfläche dar. Der durch Druckgradienten erzeugte Volumenstrom in die Sterilgutverpackung und die damit einhergehende, mögliche aerobe Keimlast verteilt sich zufällig auf der gesamten Innenfläche der Packungseinheit. Dies bedeutet wiederum, dass eindringende Keime nicht zwangsläufig auf den hierfür vorgesehenen Nährboden sedimentieren müssen. Zudem ist zu berücksichtigen, dass weitere Luftströmungen die bereits auf die Nährkartonscheibe sedimentierten Mikroorganismen durchaus wieder weiter verteilen können. Hierdurch würden Mikroorganismen auf einen Bereich innerhalb der Verpackung fallen, der keinen Nährkarton aufweist. Dadurch entziehen sich solche Mikroorganismen der Nachweisbarkeit. Ebenso ist es umgekehrt möglich, dass Keime, welche sich primär außerhalb der Petrischale befinden, sekundär durch Luftströmungen auf den Nährboden gelangen können.

Somit stellt die Betrachtung der Bewegungen bereits eingedrungener Keime durch anschließende Luftströmungen eine durchaus vernachlässigbare, sich ausgleichende Größe dar.

Wenn von einer zufälligen Verteilung eingedrungener, sedimentierter Keime ausgegangen wird, ist die Anzahl der auf der Nährkartonscheibe befindlichen Keime auf den Rest der Innenfläche der Verpackung im Verhältnis übertragbar.

Hier kann das Flächenverhältnis der genutzten Nährkartonscheibe zur durchschnittlichen Innenfläche der Papierseite der angefertigten Testeinheiten bestimmt werden.

Abb. 10:

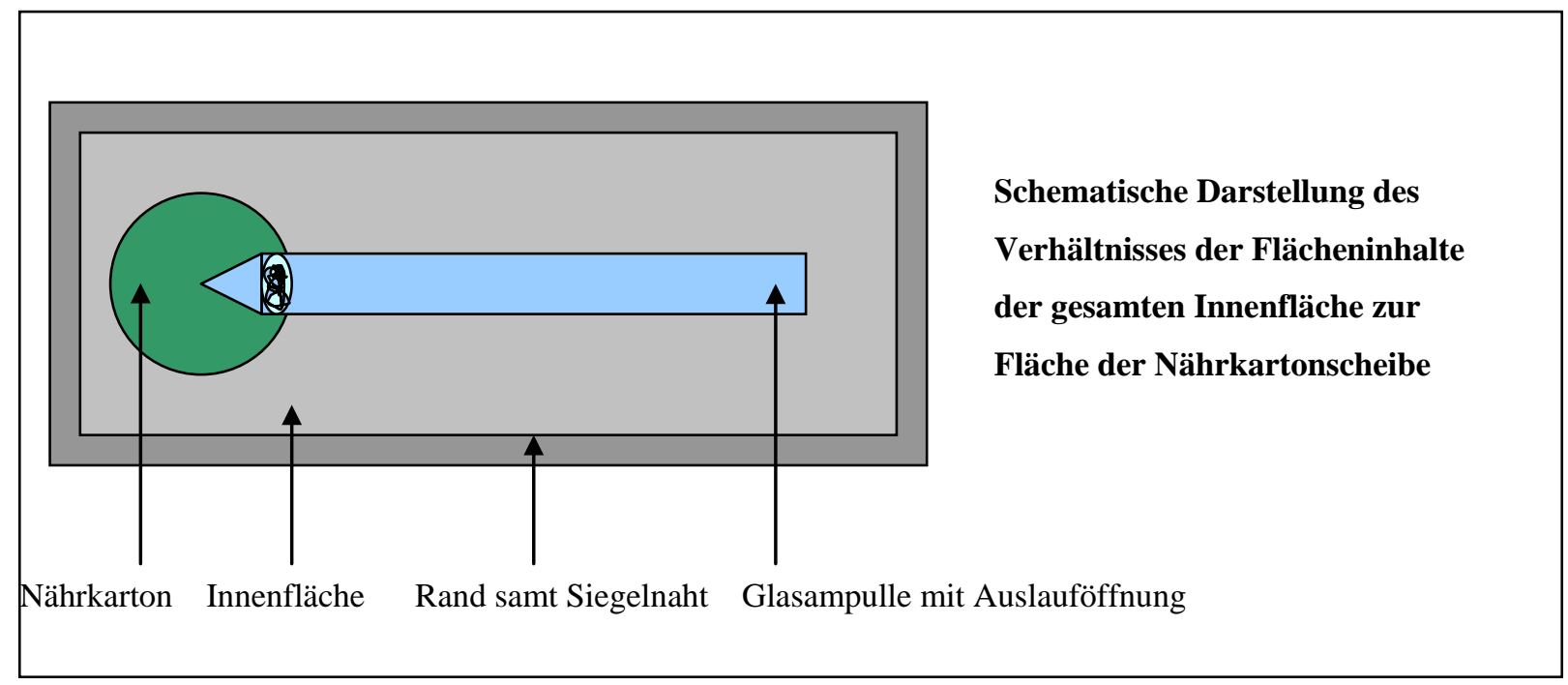




\section{Berechnungen der Flächeninhalte:}

- Durchmesser d der Nährkartonscheibe: $47 \mathrm{~mm}$

Berechnung der Fläche: $\pi * \mathbf{r}^{2}=\mathbf{1 7 3 4 , 9 4 4 5} \mathbf{m m}^{2}$

$$
\begin{aligned}
& \mathrm{d}=\text { Durchmesser } \\
& \mathrm{r}=\text { Radius }(=1 / 2 \mathrm{~d})
\end{aligned}
$$

- Maße der Innenfläche der Papierseite:

Länge durchschnittlich: $240 \mathrm{~mm}$

Breite exakt: $80 \mathrm{~mm}$

Flächeninhalt $=80 \mathbf{~ m m} * 240 \mathbf{~ m m}=19200 \mathbf{~ m m}^{2}$

\section{Berechnung der Wahrscheinlichkeit einer Sedimentation auf die Nährkartonfläche:}

- $\quad 1734,9445 \mathrm{~mm}^{2} / 19200 \mathrm{~mm}^{2}=0,09036 \ldots \rightarrow \underline{\mathbf{9 , 0 4} \%}$

\section{Schlussfolgerung:}

Die Wahrscheinlichkeit, dass ein Keim auf die nachweisbare Fläche (die Nährkartonscheibe) fällt, liegt bei circa 9,04 \%, der Keimnachweis ist demnach in < 1/10 der Fälle gegeben.

Umgekehrt bedeutet dies jedoch ebenfalls, dass bei dem Nachweis eines einzelnen Keimes auf der Nährkartonscheibe theoretisch davon ausgegangen werden kann, dass insgesamt circa weitere 9 Keime (wenn 1 Keim auf 9,04\% der Gesamtinnenfläche inklusive Nährkarton zu finden ist) ins Verpackungsinnere eingedrungen sind, sich jedoch durch zufällige Verteilung innerhalb der Verpackung der Nachweisbarkeit auf der Nährkartonscheibe entziehen.

\section{Abb. 11:}

\section{Graphische Darstellung des Flächenverhältnisses zwischen Nährkartonscheibe und der gesamten Innenfläche der Verpackung}

$\rightarrow$ Berechnung des Flächenverhältnisses als Ausdruck der Wahrscheinlichkeit einer Sedimentation von Keimen auf die Nährkartonscheibe im Hinblick auf die gesamte Innenfläche der Testeinheit (in \%)

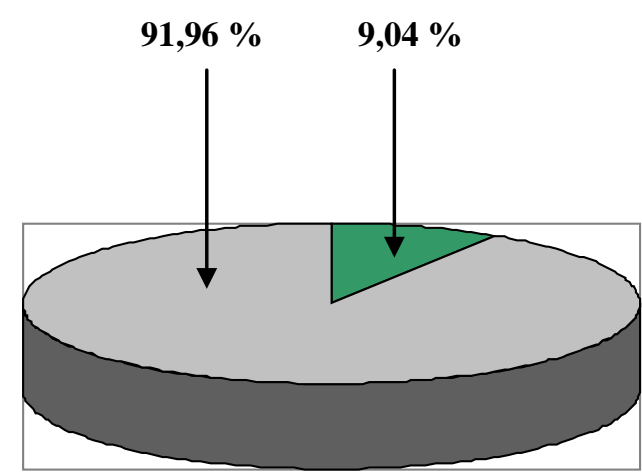

Somit wird eine Forderung bezüglich einer Optimierung der Testeinheiten ersichtlich. Die Innenfläche der Testeinheit muss durch eine ihr entsprechende, nahezu identisch große Fläche mit Nährboden abgedeckt werden. Auf diese Weise ist es möglich die korrekte Menge sedimentierter Keime, die anzüchtbar sind, zu ermitteln. 
Abb. 12:

\section{Verbesserungsentwurf der Testeinheit}

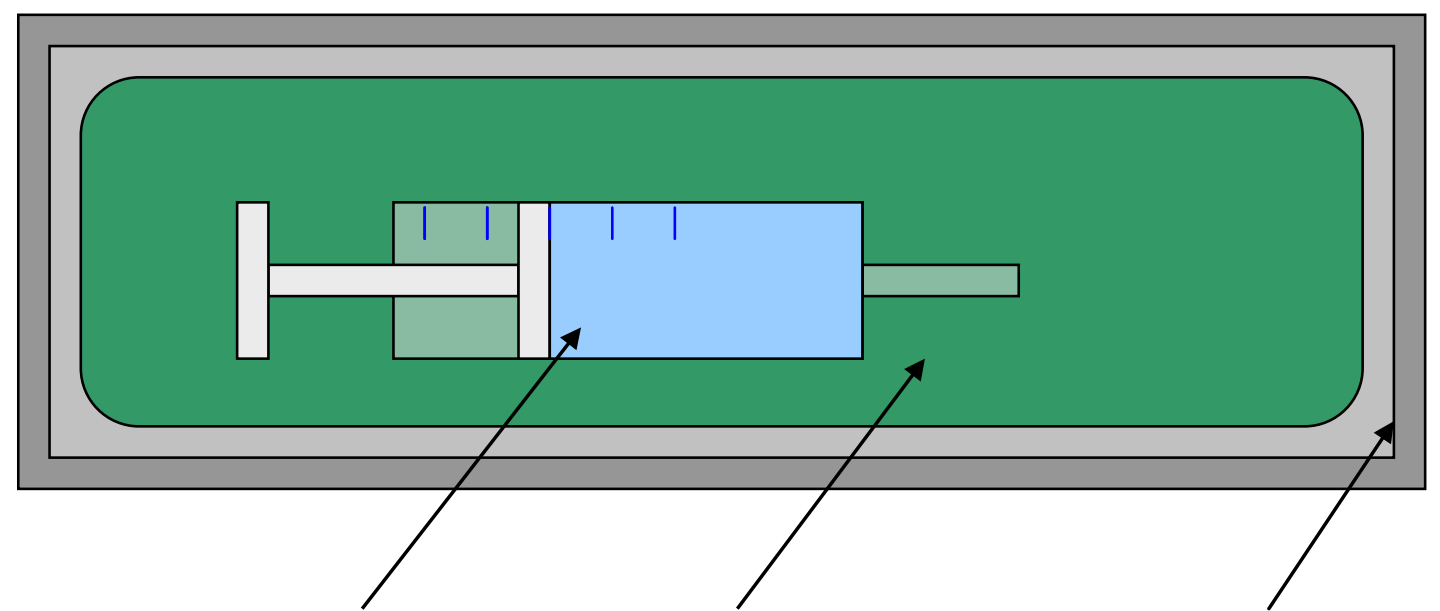

integrierte Spritze mit Wasserreservoir ～vergrößerte Nährkartonscheibe Papier-Folien-Verbundverpackung

Entscheidend bei diesem Verbesserungsentwurf ist die vergrößerte, auf die Verpackungsgröße abgestimmte Nährkartonscheibe. Hierdurch kann die Fehlerquelle bezüglich der Relation der Nährkartonoberfläche zur Innenoberfläche der Verpackung reduziert werden. Weiterhin bietet die in der Verpackung integrierte Spritze eine unkomplizierte Möglichkeit ein Wasserreservoir flexibel zur Verfügung zu stellen, ohne den bisher umständlichen Fertigungsaufwand und die schwierige Handhabung beim Öffnungsimpuls. Die Spritze müsste für den Vorgang der Dampfsterilisation thermoresistent sein und in ihrer Lage fixiert werden, um eine Schädigung der Nährkartonscheibe und die damit einhergehende Beeinflussung auf mögliche Keimnachweise zu verhindern.

\section{Nachweisbarkeit auf das Nährmedium sedimentierter Keime}

Des Weiteren ist zu berücksichtigen, dass nur vermehrungsfähige Mikroorganismen nachweisbar sind, die auf den angewandten Nährmedien bei entsprechender Zugabe von Wasser Wachstum zeigen.

Hierzu zählen die in der Umgebungsluft hoch dispers verteilten bakteriellen Endosporen und pilzbedingten Exosporen (vegetative Pilzsporen). Auf CASO- Nährkarton wachsen Bakterien und Pilze, demnach lassen sich auch Hefen auf diesem Wege durch Anzüchten auf dem Nährmedium feststellen.

Die nativen Keime (z.B. grampositive und gramnegative Bakterien), welche mit dem genutzten Nährmedium angezüchtet und nachgewiesen werden können, nehmen in diesem Fall jedoch eine Sonderstellung ein. Zwar sind diese Keime mittels der verwendeten CASO- Nährkartonscheibe nachweisbar, sterben jedoch aufgrund der langen Lagerungszeiten in den Versuchsreihen ab, bevor der biologische Teil der Testeinheiten aktiviert werden kann. Demnach sind nur jene nativen Keime nachweisbar, welche kurz vor der Aktivierung der Testeinheiten auf das Nährmedium sedimentiert sind oder weitgehend resistent gegen Austrocknung (z.B. Sporenbildner) sind. 


\section{Möglichkeit falsch positiver Ergebnisse}

Eine Möglichkeit falsch positiver Untersuchungsergebnisse mit der entwickelten Testeinheit ist lediglich denkbar, wenn die Testeinheit an sich undicht ist. Hier ist der Fokus auf die Schwachstelle der heißsielfähigen Verpackungsmaterialien zu legen. Wenn die Siegelnaht mangelhaft ist, muss ein positives Rekontaminationsergebnis als falsch positiv gewertet werden. Bei einer positiven Befundung auf Rekontamination ist es somit erforderlich die Integrität der Siegelnaht zu prüfen. Hierzu dient zum einen die optische Inspektion der Siegelnaht, minimale Defekte können sich jedoch der visuellen Detektion entziehen.

Eine ergänzende Möglichkeit zur Testung der Dichtigkeit der Schweißsiegelnaht stellt daher der Einsatz einer Farbstofftestlösung dar. Dieser Farbstofftest, auch als „Siegelnahttest“ bezeichnet, findet auch in der Lebensmittelindustrie Anwendung, um z. B. die „Invasion von Vorratsschädlingen durch Verschlüsse“ zu prüfen (Kahn 1983, S.91).

„Der Farbstoff- oder Rhodamin B- Test dient zur Überprüfung der Dichtigkeit von Siegelnähten von Verpackungen sowie stückigen Einzelverpackungen“ (Bergmair et al. 2004, S. 249).

Bei einem positiven Befund der Testeinheiten auf lebensfähige Mikroorganismen besteht somit die Möglichkeit einer zusätzlichen Testung der Verpackung im Sinne dieses „Siegelnahttestes“, bei dem mit Hilfe einer bestimmten Lösung (Rhodamin B- Lösung) die Einzelkomponente Siegelnaht überprüft werden kann. Eingebracht in die Sterilverpackung detektiert diese Flüssigkeit Löcher, Risse, Tunnelbildungen und andere Fehlstellen der Siegelnaht. „Eine gefärbte Prüflösung wird in die Verpackung gefüllt. Undichtheiten können durch Heraustreten der Prüflösung einfach optisch detektiert werden“ (Bergmair et al. 2004, S. 249).

Die in der wässrigen Rhodamin B- Lösung enthaltenen Inhaltsstoffe sind in abnehmender Konzentration wie folgt enthalten:

- $\quad$ Aqua dest.

- Isopropanol

- $\quad$ Tensid (TWEEN 80)

- $\quad$ Rhodamin B (Farbstoff).

Abb.13:

Schweißsiegelnahttest mit
Rhodamin-B-Lösung, eine
visuell nicht zu erkennende
Leckage wird detektiert

Defektleckage

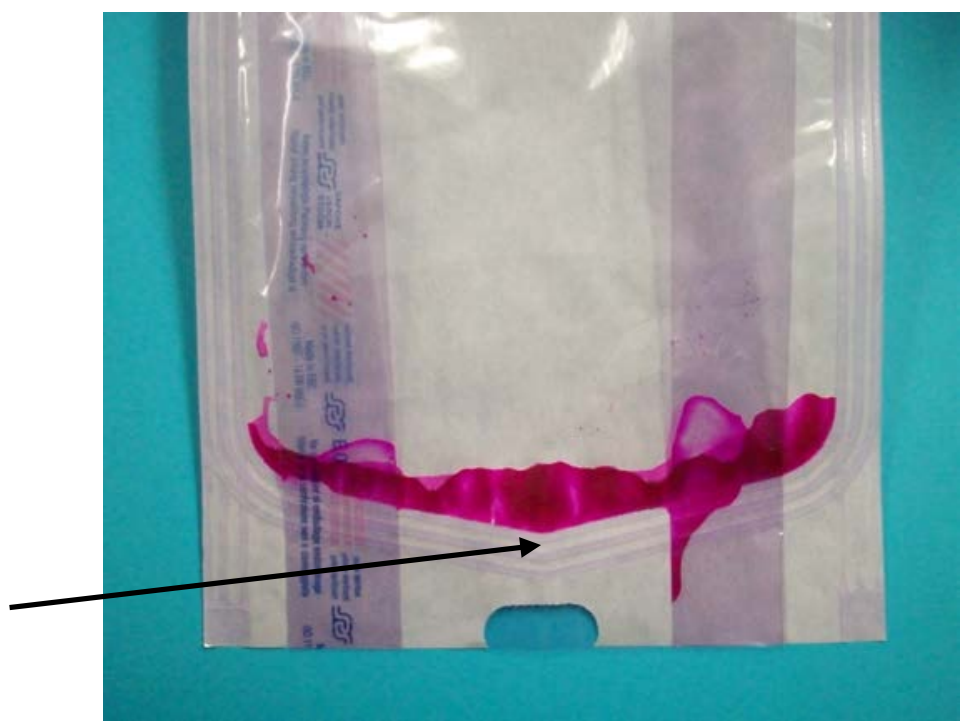




\subsection{Zusammenhang zwischen Verpackungsdesign und Verpackungsqualität}

Mit entscheidend für den durch Umgebungsbedingungen hervorgerufenen Volumenstrom ist auch das Verpackungsdesign im Hinblick auf Volumen und Beschaffenheit.

Zum Verpackungsdesign zählt jedoch nicht nur das Füllvolumen, auch die Flexibilität der Packung ist zu betrachten. Je starrer das Verpackungsmaterial gestaltet ist, desto größer ist das mögliche Luftvolumen, das fließen kann.

Bei den Sterilgutverpackungen sind hier zwei unterschiedliche Verpackungssysteme zu differenzieren. Im folgenden wird das flexible dem starren Verpackungssystem gegenübergestellt.

\subsubsection{Flexible Verpackungssysteme}

$\mathrm{Zu}$ den flexiblen Verpackungssystemen gehören zum Beispiel Papierbeutel, Textilien, Textilersatz und Sterilisationspapier wie es auch bei der mit Papier-Folien-Verbundverpackungen konstruierten Testeinheit Anwendung fand. Hierbei handelt es sich um ein System von geringem Gewicht, welches sich hervorragend dem zu sterilisierenden Produkt anschmiegen und adaptieren kann. Der effektive Verschluss der Verpackung erfolgt mittels eines Heißsiegelgerätes mit einer Siegelnahtbreite von mindestens 6 mm (DIN 58953-7 (2010)).

Da diese Verpackungen Poren aufweisen müssen, um das entsprechende Sterilisiermedium (Wasserdampf, Ethylenoxid oder Formaldehyd) penetrieren zu lassen, handelt es sich hierbei um Einwegartikel. Nach erfolgtem Sterilisationsprozess sinkt der Porendurchmesser und damit die Gasdurchlässigkeit. Hierdurch ist eine wiederholte Anwendung dieser Verpackungen nicht möglich. Die Luftdurchlässigkeit wäre bei erneuter Sterilisation nicht mehr ausreichend gewährleistet, wodurch die Verpackung im Autoklaven nicht den notwendigen Druckausgleich zwischen Verpackungsinnerem und -äußerem erlaubt und reißen würde.

Dies bedeutet somit, dass die Poren beim Prozess der Sterilisation die Penetration des Wasserdampfes ins Verpackungsinnere gewährleisten müssen, das Papier also luftdurchlässig bleibt.

Andererseits stellen genau diese zwei Faktoren bei der Lagerung des Sterilgutes eine Gefahr für das sterile Medizinprodukt dar. Denn jetzt soll die Verpackung das Eindringen von Mikroorganismen effektiv verhindern und so die Sterilität des Medizinproduktes wahren.

Folglich stellen die Luftdurchlässigkeit sowie der Porendurchmesser die zwei einzigen Parameter des Verpackungsmaterials dar, welche sowohl für die Sterilisation, als auch für die Barrierefunktion nach erfolgter Sterilisation von Relevanz sind.

Demgegenüber stehen eine Reihe an Parametern wie zum Beispiel Zugfestigkeit oder Oberflächenwiderstand des Verpackungsmaterials, welche keine oder nur eine geringe Relevanz für die Sterilisation oder Barrierefunktion haben.

Einen Überblick über die Bedeutung verschiedener Parameter auf die Sterilisation oder die Barriereeigenschaften von Verpackungen (glattes Papier oder Papier für Klarsichtbeutel) gibt Tabelle 19 auf Seite 73. 
Tabelle 19: Vorgaben zur Porosität von Verpackungen und Filtern

\begin{tabular}{|c|c|c|c|c|}
\hline & $\begin{array}{l}\text { Glattes Papier } \\
\text { (DIN EN 868-2) }\end{array}$ & $\begin{array}{l}\text { Papier für } \\
\text { Klarsichtbeutel } \\
\text { (DIN EN 868-3) }\end{array}$ & $\begin{array}{l}\text { Relevanz: } \\
\text { Sterilisation }\end{array}$ & $\begin{array}{l}\text { Relevanz : } \\
\text { Barrierefunktion }\end{array}$ \\
\hline Sichtprüfung & $\mathrm{X}$ & $\mathrm{X}$ & nein & nein \\
\hline Chemische Parameter & $X$ & $X$ & nein & nein \\
\hline Toxikol. Parameter & $\mathrm{X}$ & $\mathrm{X}$ & nein & nein \\
\hline Oberflächenwiderstand & $\mathrm{X}$ & - & nein & gering \\
\hline Weiterreißwiderstand & $\mathrm{X}$ & $\mathrm{X}$ & nein & gering \\
\hline Luftdurchlässigkeit ${ }^{1)}$ & $\begin{array}{c}1,5 \mathrm{l} / \mathrm{min}^{*} 100 \mathrm{~cm}^{2} \\
=1,7 \mu \mathrm{m} / \mathrm{Pa}^{*} \mathrm{~s}\end{array}$ & $\begin{array}{c}3,0 \mathrm{l} / \mathrm{min}^{*} 100 \mathrm{~cm}^{2} \\
=3,4 \mu \mathrm{mm} / \mathrm{Pa}^{*} \mathrm{~s}\end{array}$ & ja & ja \\
\hline Berstfestigkeit & $\mathrm{X}$ & $\mathrm{X}$ & nein & gering \\
\hline Naß- Berstfestigkeit & $\mathrm{X}$ & $\mathrm{X}$ & nein & gering \\
\hline Wasserwiderstand & $\mathrm{X}$ & $\mathrm{X}$ & nein & gering \\
\hline Porengröße & $\max .50 \mu \mathrm{m}$ & $=35 \mu \mathrm{m}$ & ja & ja \\
\hline Streckung & $\mathrm{X}$ & - & nein & nein \\
\hline Zugfestigkeit & $\mathrm{X}$ & $\mathrm{X}$ & nein & nein \\
\hline Naßfestigkeit & $\mathrm{X}$ & $\mathrm{X}$ & nein & gering \\
\hline
\end{tabular}

(Quelle: Dunkelberg und Schmelz; Universitätsmedizin Göttingen

URL http://www.sterilgutverpackung-

bewerten.de/5e65d9c1a46cab8c47ea8d4cd33679d2_Infomaterial_Haupttext.pdf_)

[Eingesehen am 13.04.2012; 14:23 Uhr]

\subsubsection{Starre Verpackungssysteme}

Dem flexiblen Verpackungssystem gegenüberzustellen sind die starren Verpackungen (Sterilgutcontainer).

Das Sterilisationsmedium (Wasserdampf, Ethylenoxid oder Formaldehyd) muss ins Innere der Container gelangen, um hier seine Wirkung zu entfalten. Gewährleistet wird dies durch effektive Filter- oder Ventilsysteme, welche mit Textilien, Teflon oder Papier beschickt sind. Eine Kontamination nach erfolgtem Sterilisationsprozess soll durch diese Ventilsysteme und zusätzliche Dichtungen und Verplombungen unterbunden werden. Es handelt sich bei diesen Containern nicht um Einwegverpackungen. Bei jedem erneuten Gebrauch müssen lediglich die eingelegten Filterpapiere oder Textilien ausgetauscht werden. Teflonfilter sind sogar mehrfach verwendbar (bis zu 1000 Sterilisierzyklen). Die Funktionstüchtigkeit des Ventilsystems ist Voraussetzung für die sichere Sterilisation und Lagerung von Medizinprodukten.

Verbrauchsspuren, die mit dem ständigen Gebrauch der Container einhergehen, wie zum Beispiel defekte Ventilsysteme, undichte Verplombungen oder aber auch spröde Dichtungen stellen einen Risikofaktor für eine Rekontamination dar.

Eine Möglichkeit der Testung häufig genutzter Container und somit eine Kontrolle der Effektivität ihrer Barrierewirksamkeit wurde bereits entwickelt und durchgeführt. In der Studie „Measurement of the microbial barrier effectiveness of sterilization containers in terms of the log reduction value for prevention of nosokomial infections“ wird ein Testverfahren dargestellt, durch welches Sterilgutcontainer regelmäßig auf ihre Eignung im medizinischen Alltag geprüft werden können (Dunkelberg und Fleitmann- Glende 2006, S. 285) .

Hierzu wurden die Container im Expositionskammerverfahren definierten Druck- und Temperaturschwankungen sowie einem keimhaltigen Aerosol ausgesetzt. Mittels einer eigens hierfür entwickelten Methode konnte der Container zur Anzüchtung eingedrungener Keime verschlossen bleiben. 
Hierdurch wurde der Einstrom von Keimen durch einen beim Öffnen des Containers verursachten Volumenstrom verhindert.

Nach durchlaufener Überprüfung konnte die Barrierewirksamkeit eines Containers abschließend als dezimallogarithmische Keimreduktion (logarithmischer Reduktionsfaktor, LRF) angegeben werden.

„An LRV of >4 should be envisaged as the target assurance level“ (Dunkelberg und Fleitmann- Glende 2006, S. 285).

Abb. 14:

\begin{tabular}{l}
\hline Darstellung der Bestandteile eines \\
Sterilgutcontainers \\
Quelle: \\
redam- instrumente GmbH $\quad$ - \\
chirurgie/medizintechnik/containersysteme \\
URL \\
http://www.redam-instrumente.de/ \\
container-siebkoerbe.html \\
[Eingesehen am 24.05.2010; 16:22 Uhr] \\
\end{tabular}

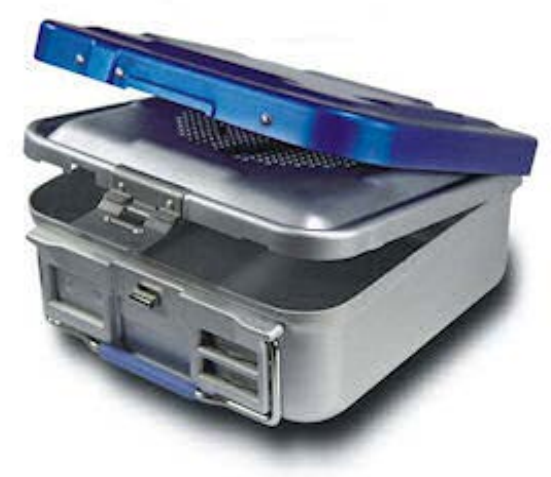

4.6 Einfluss der mikrobiologischen Beanspruchung auf die Wahrung der Sterilität bei verschiedenen Lagerungsbedingungen

Nicht nur das Verpackungssystem an sich trägt zur Wahrung der Sterilität bei, sondern auch die vorgeschriebene Handhabung und die nach dem Deutschen Institut für Normung (DIN 58953-8 (2010) und DIN 58953-9 (2010)) vorgegebenen Richtlinien zur Lagerungsdauer bei gegebener Lagerungsart.

Zum einen wird hier zwischen ungeschützter Lagerung (offen gelagert, nicht in Schränken) und geschützter Lagerung (in verschließbaren Schränken) differenziert. In Schränken ist das Sterilgut vor mechanischer und chemischer Schädigung geschützt.

Zum anderen ist die Örtlichkeit der Lagerung an sich zu berücksichtigen. Eine hohe Umgebungskeimlast erhöht das Risiko einer Rekontamination des verpackten Medizinproduktes.

Die Rekontamination der Testeinheiten erfolgte durch den Einstrom von in der Luft enthaltenen Keimen. Hierbei war es unumgänglich die Keimlast der umgebenden Atmosphäre einzuschätzen.

Die Ergebnisse dieser Arbeit legen die Schlussfolgerung nahe, dass eine niedrige Keimlast der einströmenden Luft folglich mit einer geringeren Gefahr der Rekontamination der Testeinheiten einhergeht, während eine besonders hohe Keimlast der Luft eine stärkere Kontamination bei gleichem Volumenaustausch nach sich zieht.

Die Betrachtung der in der entsprechenden Lagerungsatmosphäre enthaltene Keimlast wird in $\mathrm{KbE} / \mathrm{m}^{3}$ angegeben. Es handelt sich hierbei um koloniebildende Einheiten pro Kubikmeter Luft. 
Durch die verschiedenen Versuchsreihen, die zum einen in Bad Rothenfelde und in Oldenburg und zum anderen in den unterschiedlichen Kornhäusern (Vorversuche 2006) durchgeführt wurden, konnte eine Abhängigkeit der Rekontamination von der aerogenen mikrobiellen Beanspruchung nachgewiesen werden.

In Bad Rothenfelde handelte es sich um die Exposition der Testeinheiten unter Reinluftbedingungen in Nähe der Operationssäle.

Die in Oldenburg deponierten Testeinheiten befanden sich in einer üblichen Lagerhalle ohne zusätzliche raumlufttechnische Aufbereitung.

„S Sedimentieren lebendige, vermehrungsfähige Mikroorganismen aus der Umgebungsluft auf einer Risikofläche, so entsteht Kontaminationsgefahr“ (Eidgenössische Materialprüfungs- und Forschungsanstalt 2004, URL http://www.empa.ch/plugin/template/empa/*/27408/---/1=1 ; Stand: 21.06.2011; 21:08 Uhr).

Demgegenüber ist die Lagerung der Testeinheiten in den verschiedenen Kornhäusern zu sehen. Sowohl in Dransfeld und Obernjesa als auch in Scharzfeld ist die Belastung der Luft durch Mikroorganismen wesentlich höher. Man kann in diesem Fall von einer Sättigung der Atmosphäre mit Keimen sprechen, welche mit bloßen Auge als Trübung der Luft auffällt.

\section{Welche Faktoren beeinflussen die Lufthygiene?}

\section{- Schleusensysteme}

$\rightarrow$ Hier wird die Reinluft effektiv von der „üblichen Luft“(Umgebungsluft) getrennt, zudem können im Reinraumbereich ca. 3 bis 5 hPa höhere Luftdrücke eingerichtet werden, sodass bei Öffnung der Türen Reinluft nach außen strömt, jedoch das Einströmen von unreiner Außenluft vermieden wird.

- Personal

$\rightarrow$ Sowohl die Ausbildung des Personals als auch deren gewissenhafte Arbeit mit genauen Vorschriften verbessert die Lufthygiene.

\section{- Infrastruktur}

$\rightarrow$, Je intensiver die Aktivitäten und je länger sie dauern, desto größer wird die Anzahl der Keime in der Luft“ (Eidgenössische Materialprüfungs- und Forschungsanstalt 2004, URL http://www.empa.ch/plugin/template/empa/*/27408/---/1=1 ; Stand: 21.06.2011; 21:08 Uhr).

\section{- Luftreinigungsanlagen}

$\rightarrow$ Effektive Filtrationsverfahren (z.B. HEPA-Filter) zur Keimzahlminimierung finden nicht nur im medizinischen Bereich, sondern auch in der Lebensmittelhygiene Anwendung.

\section{- Mikrobielle Luftüberwachung}

$\rightarrow$ Die Untersuchung von Luftproben auf Nährmedien mittels Luftkeimsammelgerät ist ein sinnvolles Hilfsmittel.

- Weitgehende Keimfreiheit der Oberflächen durch die Anwendung zugelassener Flächendesinfektionsmittel. 


\subsection{Die Problematik der Filterlücke}

Einen weiteren, bisher lediglich kurz in der Einleitung (siehe Abschnitt 1.2, Seite 9 - 11) erwähnten Faktor stellt die Anwendung von porösem Sterilisationspapier mit seinen spezifischen Eigenschaften im Sinne einer Barrierefunktion als Verpackungsmaterial für Medizinprodukte dar.

Um im Hinblick auf Sterilgutverpackungsmaterialien europaweit geltende Normen aufstellen zu können, musste zunächst eine treffende Bezeichnung für diese Verpackungssysteme gefunden werden, um „den Grundlegenden Anforderungen der Europäischen Richtlinie über Medizinprodukte zu entsprechen“ (DIN EN ISO 11607-2 (2006) S.4). Aus diesem Bestreben erfolgte die Einführung des Terminus „Sterilbarrieresystem“. Bindende Anforderung an dieses „Sterilbarrieresystem“ ist es, „eine Sterilisation zu ermöglichen, eine akzeptable mikrobielle Barriere darzustellen und die aseptische Bereitstellung des Produkts zu ermöglichen“ (DIN EN ISO 11607-2 (2006) S.4).

Vereinfacht betrachtet repräsentiert dieses Prinzip ein Filtersystem.

Um das Prinzip eines Filterpapiers bezüglich der Barrierewirkung zu verstehen, ist es unumgänglich, sich mit dessen Aufbau und Funktionsweise zu befassen. Bei Papier zum Verpacken und Sterilisieren von Medizinprodukten ist ein maximaler Porendurchmesser von $35-50 \mu \mathrm{m}$ erlaubt und erforderlich, um die Penetration des Sterilisiermediums Wasserdampf (oder Ethylenoxid) zum Medizinprodukt zu gewährleisten. „Bei der Prüfung [...] muss der durchschnittliche Wert des Porendurchmessers der zehn Prüflinge kleiner oder gleich $35 \mu \mathrm{m}$ sein. Keiner der Werte darf größer als 50 um sein“ (DIN EN 868-3 (2009) S.7).

Entgegen der trügerischen Annahme, dass das Filterpapier nur diejenigen Partikel abfange, welche die maximal erlaubte Porengröße von vereinzelt $50 \mu \mathrm{m}$ überschreiten, spielen hier weitaus mehr Faktoren eine entscheidende Rolle. Hierzu ist es notwendig, wichtige physikalische, das Filterpapier betreffende Größen zu definieren und die daraus resultierenden Eigenschaften des Papiers darzustellen.

Zum einen werden Partikel mit einem besonders großen Durchmesser aufgrund von Massenträgheit und durch Sperreffekte vom Sterilgut abgehalten. Die Massenträgheit stellt eines der fundamentalen Bestandteile der klassischen Mechanik dar und ermöglicht somit die Abscheidung durch Impaktion. Die Massenträgheit ist definiert als die Besonderheit von Körpern, in ihrem Bewegungszustand zu verbleiben, solange keine Kräfte auf diesen Körper einwirken. Dieses physikalische Prinzip macht sich das Filtersystem zu Nutze, indem Partikel an Faserstrukturen des Papiergewebes hängen bleiben und abgestoppt werden. Des Weiteren führt der Sperreffekt zu einer Adhäsion großvolumiger Partikel an den Faserstrukturen des Filterpapiers und somit kann ein Eindringen in die Papier- Folien-Verbundverpackung unterbunden werden.

Auf der anderen Seite werden jedoch auch Partikel abgehalten, welche einen wesentlich kleineren Durchmesser aufweisen als die Poren in der Filterpapierstruktur. Dieser zunächst paradox klingende Sachverhalt erklärt sich durch folgendes physikalisches Prinzip:

Das Phänomen der Diffusion hat seinen Ursprung in der temperaturabhängigen Eigenbewegung von Teilchen mit dem Resultat einer gleichmäßigen Durchmischung verschiedener Stoffe oder aber gleichmäßiger Verteilung eines Stoffes. Dies bedeutet wiederum, dass in einem abgeschlossenen System durch Diffusionseffekte vorhandene Konzentrationsgradienten reduziert werden, was in seinem Endzustand zu einem Stadium völliger Durchmischung führt. 
Entscheidend für die Papier-Folien-Verbundverpackung ist hierbei das Phänomen, dass kleine Teilchen wie Atome, Moleküle oder auch Keime eine Eigenbewegung abhängig von thermischen Veränderungen der Umgebung aufweisen. Das heißt, dass sich temperaturabhängig Bewegungszustände verändern. Diese Begebenheit ist als Brown`sche Molekularbewegung bekannt. Dies bedeutet für das Durchdringen eines Filterpapiers, dass kleinste Teilchen nicht etwa gradlinig die Löcher in der Faserstruktur der Papierseite durchdringen, sondern durch Zusammenstöße mit anderen Molekülen und die Eigenbewegung ständig in ihrer Richtung abgelenkt werden. Zwar ist die Größe der Partikel somit kleiner als der Porendurchmesser des Papiers, ihre dreidimensionalen Bewegungen führen jedoch in aller Wahrscheinlichkeit zu einem Aufprall an den Papierfasern, wo sie durch Adhäsion haften bleiben oder aber in eine andere Richtung abgelenkt werden. Diese durch einen Zusammenprall zufällig entstandene Richtungsänderung und somit Bewegung wird als Random Walk bezeichnet.
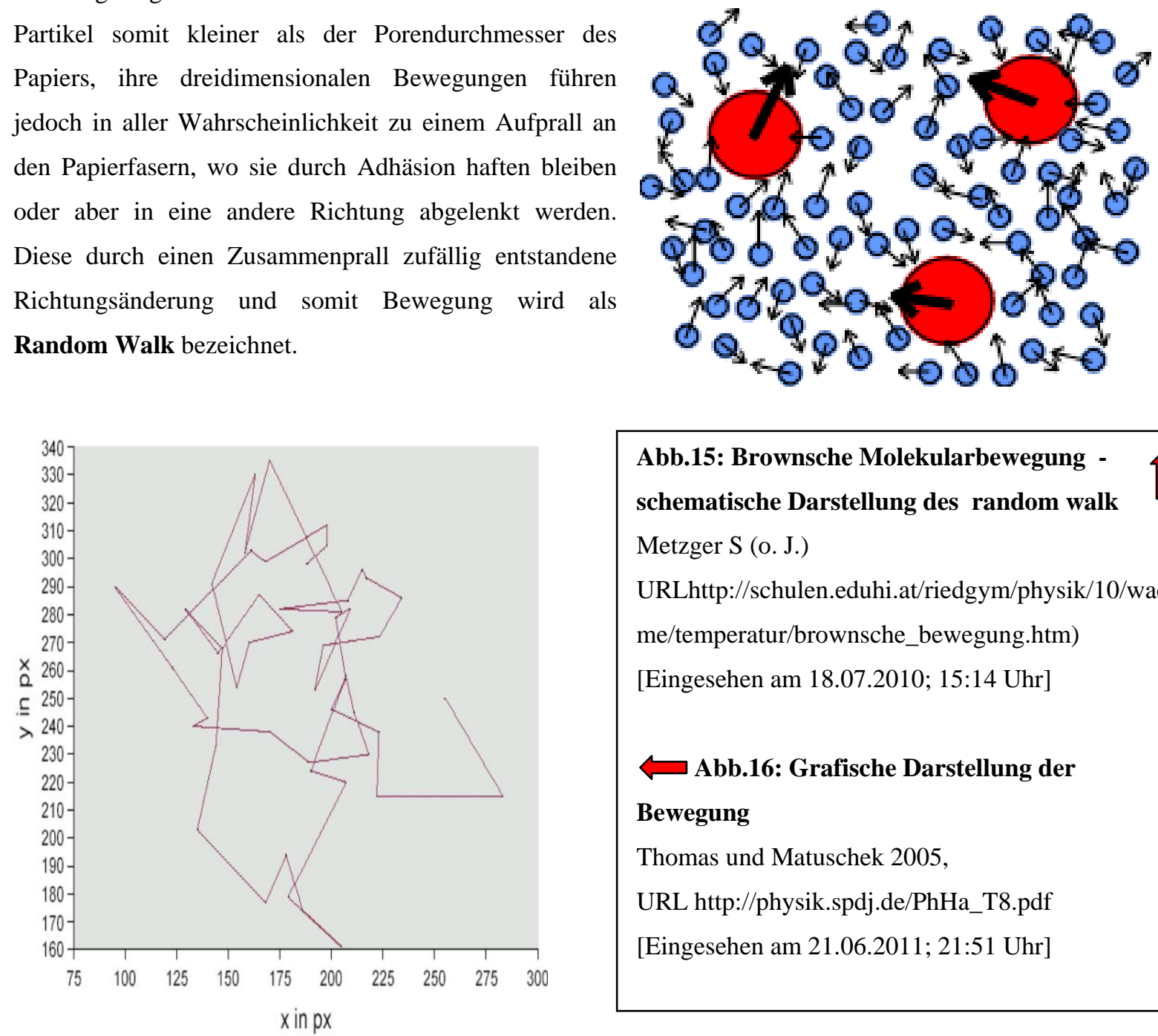

Abb.15: Brownsche Molekularbewegung -
schematische Darstellung des random walk
Metzger S (o. J.)
URLhttp://schulen.eduhi.at/riedgym/physik/10/waer
me/temperatur/brownsche_bewegung.htm)
[Eingesehen am 18.07.2010; 15:14 Uhr]
Bewegung
Thomas und Matuschek 2005,
URL http://physik.spdj.de/PhHa_T8.pdf
[Eingesehen am 21.06.2011; 21:51 Uhr]

Demzufolge kann die Struktur des Filterpapiers sehr große Partikel und sehr kleine Partikel oder Moleküle während der Lagerungsdauer effektiv vom Sterilgut abhalten und dieses vor einer Kontamination schützen.

Problematisch stellt sich jedoch der Sachverhalt dar, dass vor allem in dem Bereich der Gasfiltration eine sogenannte Filterlücke vorliegt. Bei dieser Filterlücke handelt es sich um einen Partikelgrößenbereich, in welchem entsprechende Moleküle oder Partikel durch das Filtersystem nicht ausreichend gut abgefangen werden können. Ursächlich hierfür ist, dass diese Partikelgrößen für Abscheidung durch Impaktion zu gering und für Diffusionseffekte zu groß sind. Somit können die physikalischen Filtermethoden in diesen Fällen nicht effektiv genug wirken. 
Abb. 17:

\section{Motorlexikon}

Darstellung der physikalischen

Phänomene eines Papierfilters anhand einer einzelnen Faser

URL

http://www.motorlexikon.de/?I=5441

[Eingesehen am 12.03.2011; 19:34 Uhr]

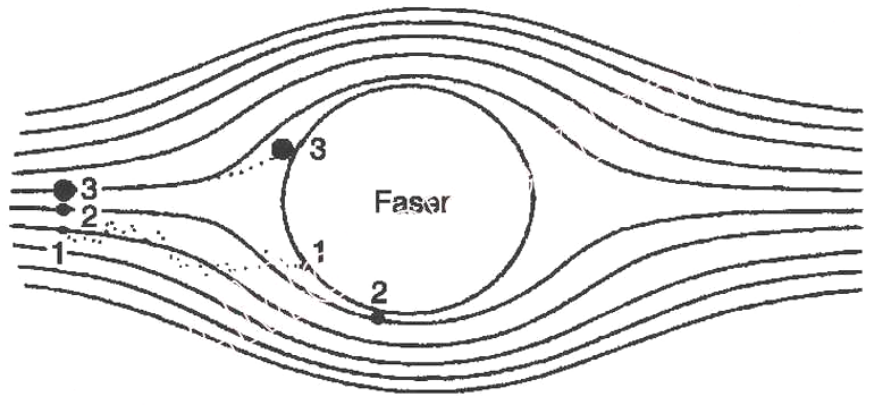

$\begin{array}{lll}\text { 1. Diffusion } & \text { 2. Abfangen } & \text { 3. Trägheitsabscheidung }\end{array}$

Abb. 18:

\section{Motorlexikon}

\section{Graphische Darstellung der Filterlücke}

(Die Filterlücke ist die Fläche unterhalb des Schnittpunktes der Diffusions- und Induktionsfunktion)

Partikel in diesem Größenordnungsbereich können nur unzureichend vom Filtersystem abgeschieden werden.

URL

http://www.motorlexikon.de/?I=5441

[Eingesehen am 12.03.2011; 19:34 Uhr]

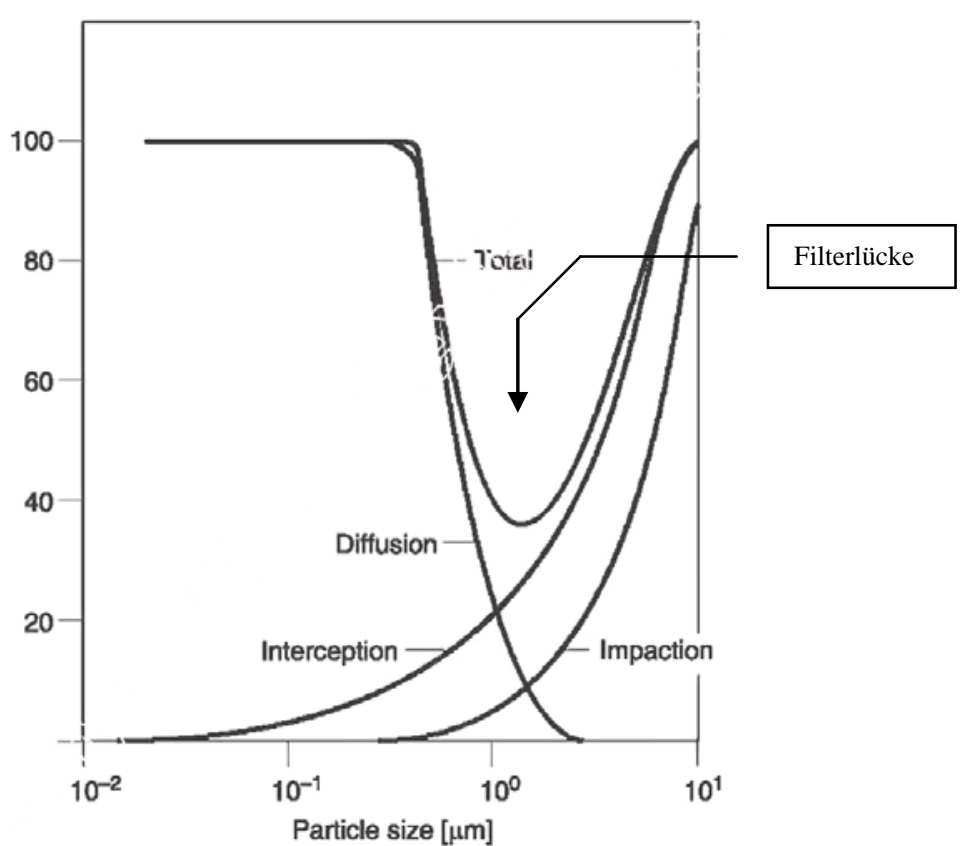

Neben der Problematik der Filterlücke an sich kommt erschwerend hinzu, dass es sich bei dem fraglichen Partikelgrößenbereich genau um das Größenspektrum handelt, in welchem sich die üblichen aerogenen, pathogenen Mikroorganismen bewegen. Durch die Filterlücke werden also Partikel in einer Größenordnung von circa 1 - $5 \mu \mathrm{m}$ nur unzureichend vom Verpackungsmaterial abgehalten. 
In diesem Zusammenhang ist es sinnvoll, sich noch einmal mit den in dem Worst-Case-Szenario Anwendung findenden Testsystemen zu beschäftigen. Bei den im Vorversuch in den Kornhäusern deponierten Testeinheiten konnten nach mikrobiologischer Aktivierung und Inkubation folgende Schimmelpilzgattungen nachgewiesen werden:

Abb. 19:

\section{Cladosporium sp.}

Sporendurchmesser: $0,5-3,5 \mu \mathrm{m}$

Darstellung der Gattung Cladosporium mithilfe eines

Durchlichtmikroskops

(400 fache Vergrößerung)

Quelle:

Szabo E 2007, S.133

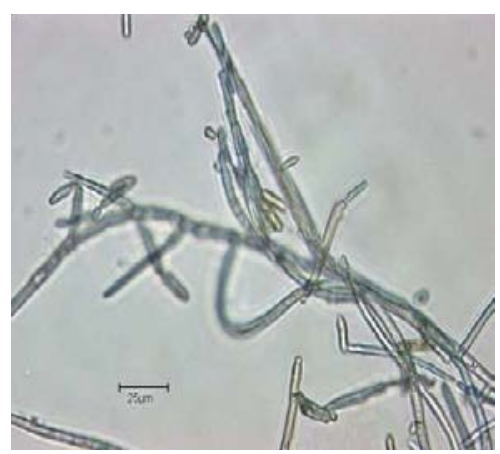

Abb. 20:

\section{Rhizopus sp.}

Sporendurchmesser: $<9 \mu \mathrm{m}$

Darstellung des Rhizopus stolonifer im REM

(1250 fache Vergrößerung)

Quelle: URL

http://www.schimmel-schimmelpilze.de/rhizopus.html

[Eingesehen am 13.03.2011; 16:07 Uhr]

Abb. 21:

\section{Penicillium sp.}

Sporendurchmesser: $1,0-3,5 \mu \mathrm{m}$

Darstellung der Gattung Penicillium mithilfe eines

Durchlichtmikroskops

(400 fache Vergrößerung)

Quelle:

Szabo E 2007, S. 134

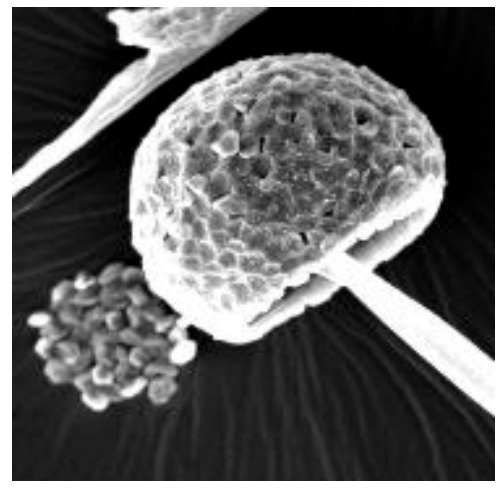

Anzumerken ist im Hinblick auf die Problematik der Filterlücke, dass diese Schimmelpilzgattungen Sporen produzieren, welche mit ihrem Durchmesser genau in den Partikelgrößenbereich fallen, der von der Papierseite der Papier-Folien-Verbundverpackung nur unzureichend abgehalten werden kann. 
Gerade unter Berücksichtigung der Tatsache, dass immer mehr ältere, multimorbide und immungeschwächte Patienten behandelt werden, stellen diese möglicherweise das Sterilverpackungsmaterial durchdringenden Partikel eine besondere Gefahr für opportunistische Infektionen dar.

„In der Regel geht von den ubiquitär vorkommenden Schimmelpilzen für den gesunden und immunkompetenten Menschen keine Gefahr aus. Ist aber das Abwehrsystem z.B. durch HIV Infektion, Autoimmunerkrankungen, Diabetes mellitus, Lungenerkrankungen, Malignome oder nach Organtransplantationen geschwächt, können Schimmelpilze eine Gesundheitsgefahr darstellen“ (Schenke S 2009, S.16).

\subsubsection{MPPS (most penetrating particle size)}

Bei der Prüfung eines Filterelementes nach EN 1822-3 muss der Fraktionsabscheidegrad gemessen werden. Anhand eines Partikelzählverfahrens kann eine Konzentrationsbestimmung durchströmender Partikel unterschiedlicher Größen ermittelt werden.

Daraus ergibt sich eine Fraktionsabscheidegradkurve des Filtersystems.

\section{Abb.22:}

Darstellung des Fraktionsabscheidegrades

\begin{tabular}{l|}
\hline $\begin{array}{l}\text { Durchlassgrad } \\
\text { in } \%\end{array}$ \\
\hline
\end{tabular}

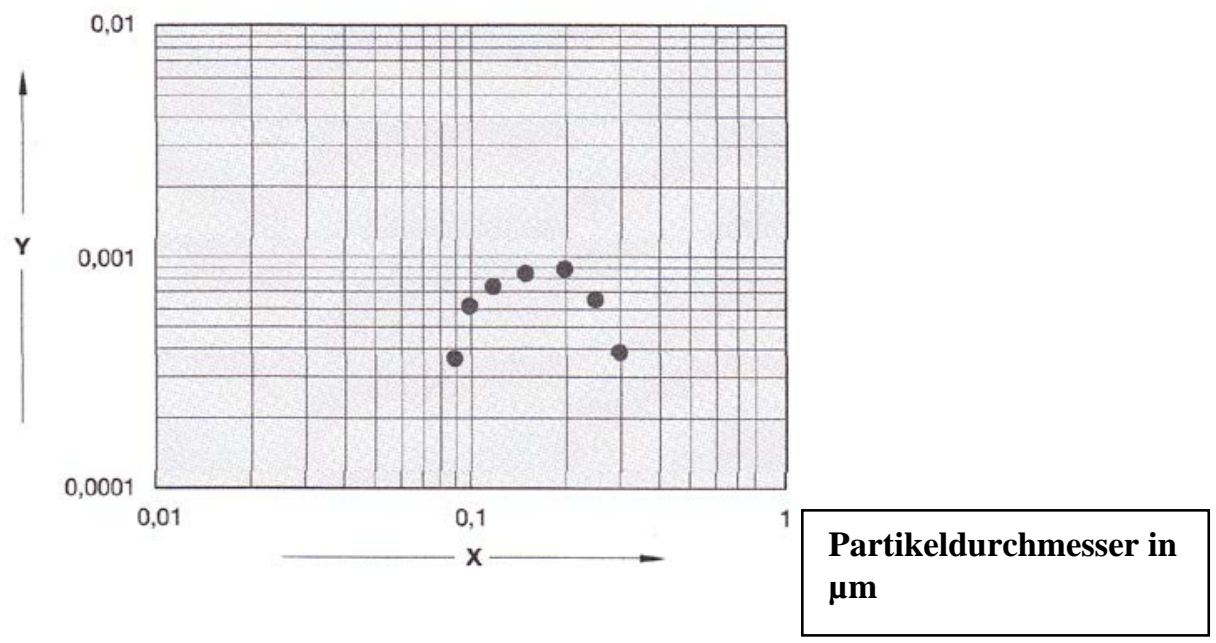

Abb. 22: Darstellung des Fraktionsabscheidegrades

$\mathrm{X}$-Achse $=$ Partikeldurchmesser in $\mu \mathrm{m}$

Y-Achse $=$ Durchlassgrad in \%

Diese Kurve zeigt einen Partikelgrößenbereich auf, für welchen der Filter am durchlässigsten ist (Filterlücke). Der Durchlassgrad weist genau für diesen Partikelgrößenbereich einen Maximalwert auf. Somit stellt der Partikelgrößenbereich, für welchen die maximale Durchlässigkeit des Filters definiert ist, die MPPS (most penetrating particle size) dar.

Quelle:

Luftfilter- Prüfverfahren zur Prüfung von Schwebstofffiltern

(C)TROX Filter GmbH 2009

URL http://www.trox.de/xpool/download/de/technical_documents/filters/leaflets/P_2_EN1822.pdf

[Eingesehen am 05.09.2012; 18:10 Uhr] 
Die Relevanz dieses Größenbereichs von Partikeln wird bei einem Blick auf die EN 1822-4 mit folgender Anforderung an das Prüfaerosol deutlich. Bei dem hier beschriebenen Luftfilter- Prüfverfahren zur Detektion von Leckagen im Filter muss das eingesetzte Prüfaerosol der mittleren Partikelgröße der MPPS entsprechen. Eine Prüfung mit diesen Partikeln (MPPS) stellt somit ein simuliertes „Worst-Case-Szenario“ für das Filterelement dar.

Bei Schwebstofffiltern erfolgt die Prüfung daher mit einer mittleren Partikelgröße von 0,2 $\mu$ m.

\section{Abb.23:}

Zunehmende Bedeutung fand dieses Thema (bzw. die Schwachstelle von Filtersystemen) in den letzten Jahren auch durch das Auftreten neuer luftgetragener, hochpathogener Infektionen wie z.B. SARS (Severe Acute Respiratory Syndrome) oder auch der Vogelgrippe HPAI (Highly Pathogenic Avian Influenza) im Hinblick auf den Mund- NasenSchutz als Filter bzw. Barriere. Die Abscheideminima liegen bei dieser Darstellung in einem Partikelgrößenbereich von 0,15 bzw. 0,2 $\mu \mathrm{m}$.

Dies ist also der Größenbereich der MPPS.

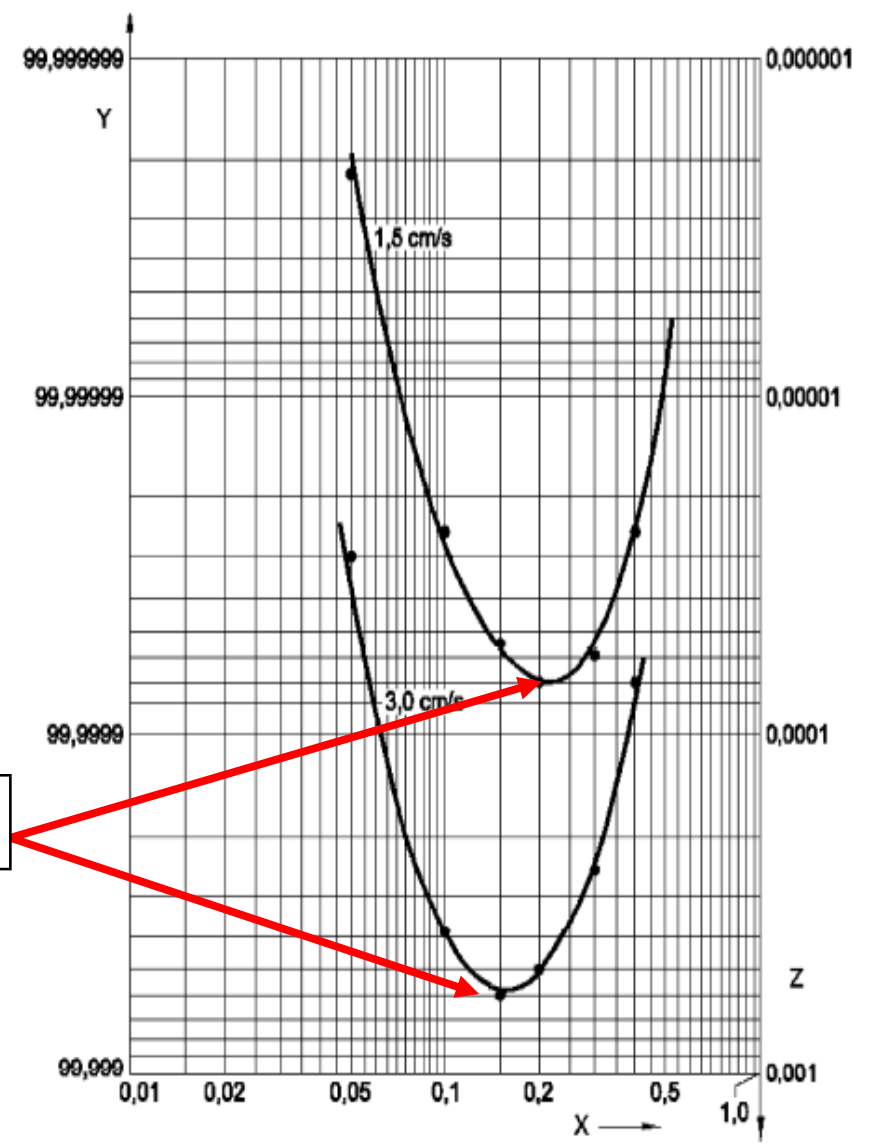

Abb. 23:

\section{Funktion des Partikeldurchmessers $d_{p}$ für zwei verschiedene Filtermediumsgeschwindigkeiten}

(Y) $\mathrm{E}=$ Fraktionsabscheidegrad

(Z) $\mathrm{P}=$ Fraktionsdurchlassgrad

(X) $d_{p}=$ Partikeldurchmesser

MPPS = most penetrating particle size

Filtermediumsgeschwindigkeiten: $1,5 \mathrm{~cm} / \mathrm{s}$ bzw. $3,0 \mathrm{~cm} / \mathrm{s}$

Quelle:

DIN EN 1822-1 2009, S.14 
Mit der Problematik der MPPS (most penetrating particle size) musste sich auch Otani et al. 2007 auseinandersetzen, um die Möglichkeit zu beurteilen, Partikel (und vor allem Nanopartikel) durch Anwendung von Luftfiltern zu klassifizieren.
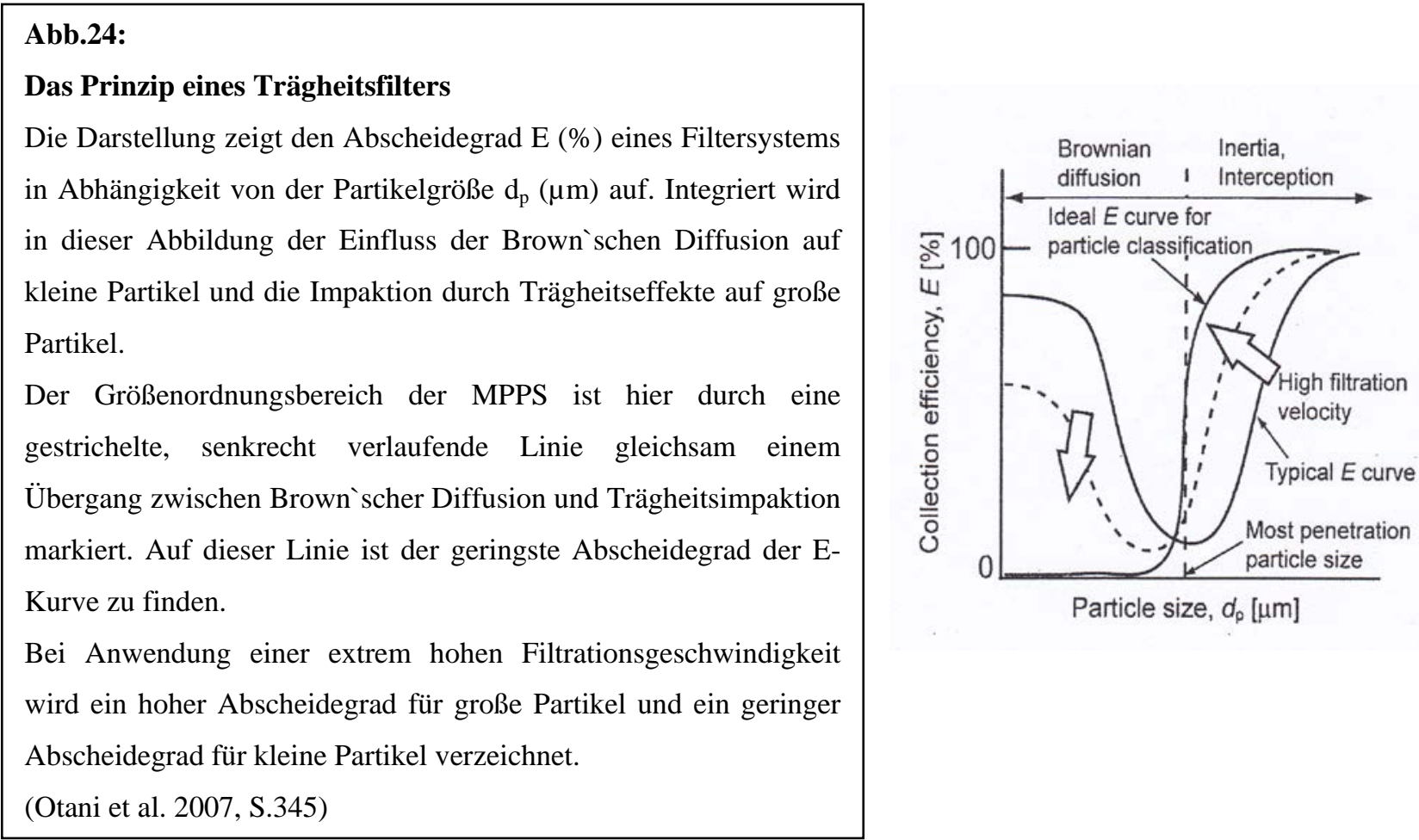

Diese hier beschriebene Most Penetrating Particle Size stellt jedoch keine definierte Größe für ein bestimmtes Verpackungsmaterial dar. Vielmehr hängt der Größenbereich der MPPS noch von weiteren die Sporenbeziehungsweise Partikelpenetration beeinflussenden Faktoren ab.

Diese Überlegungen wurden in der Veröffentlichung „Definition of a Correlation Between Microbiological and Physical Particulate Barrier Performances for Porous Medical Packaging Materials“ von Sinclair und Tallentire (Sinclair und Tallentire 2002, S. 11-19) thematisiert.

Im Rahmen ihrer Untersuchungen wurden verschiedene kommerzielle Sterilgut- Papierverpackungen unterschiedlichen Faktoren wie sporenbelastetem Aerosol, dem Prüfaerosol DEHS (unbelebte Partikel), schwankenden Druckverhältnissen oder auch diversen Luftdurchströmungsraten ausgesetzt und somit auf ihre Barriereeigenschaften getestet.

Zunächst wurde ein Sterilgutpapier schwankenden Durchflussraten eines sporenbelasteten Aerosols ausgesetzt. In der folgenden Grafik wird die Sporenpenetration als Funktion der Durchflussrate in einem logarithmisch angelegtem Diagramm dargestellt.

Abb.25:

Grafische Darstellung der prozentualen \% Sporenpenetration in Abhängigkeit von der Durchflussrate sporenbeladener Luft

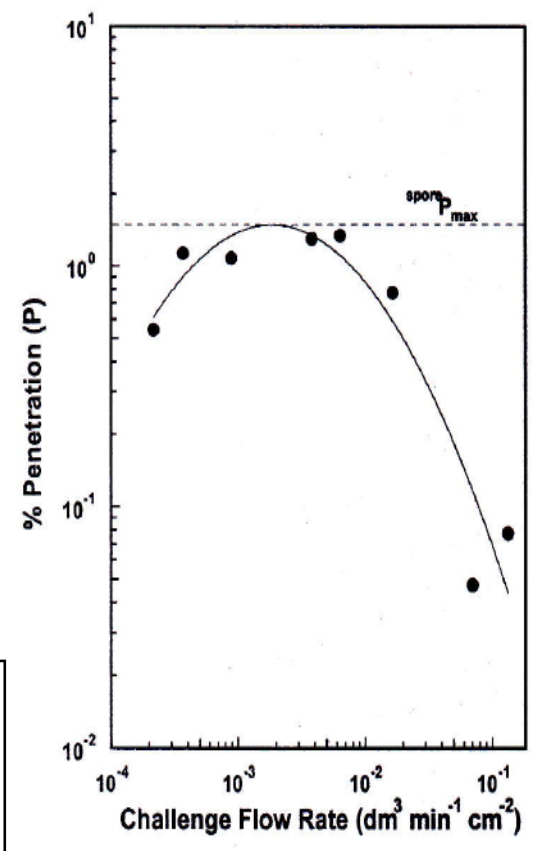

(Sinclair und Tallentire 2002, S.15) 
Die prozentuale Sporenpenetration nimmt mit ansteigender Durchflussrate zunächst zu und steigt bis zu der im Diagramm gestrichelten Linie, der sogenannten maximalen Sporenpenetration $\left({ }^{\text {spore }} \mathrm{P}_{\max }\right)$, an. Bei weiter ansteigender Durchflussrate hingegen sinkt die prozentuale Sporenpenetration wieder ab.

Dieses gegenläufige Verhalten der Sporenpenetration bei stetig steigender Durchflussrate erlaubt es, eine Partikelflussrate zu bestimmen, bei der die prozentuale Sporenpenetration maximal ist $\left({ }^{\text {spore }} \mathrm{P}_{\max }\right)$.

Die folgende Darstellung zeigt einen Partikelgrößenbereich auf, für den die Partikelpenetration am höchsten ist.

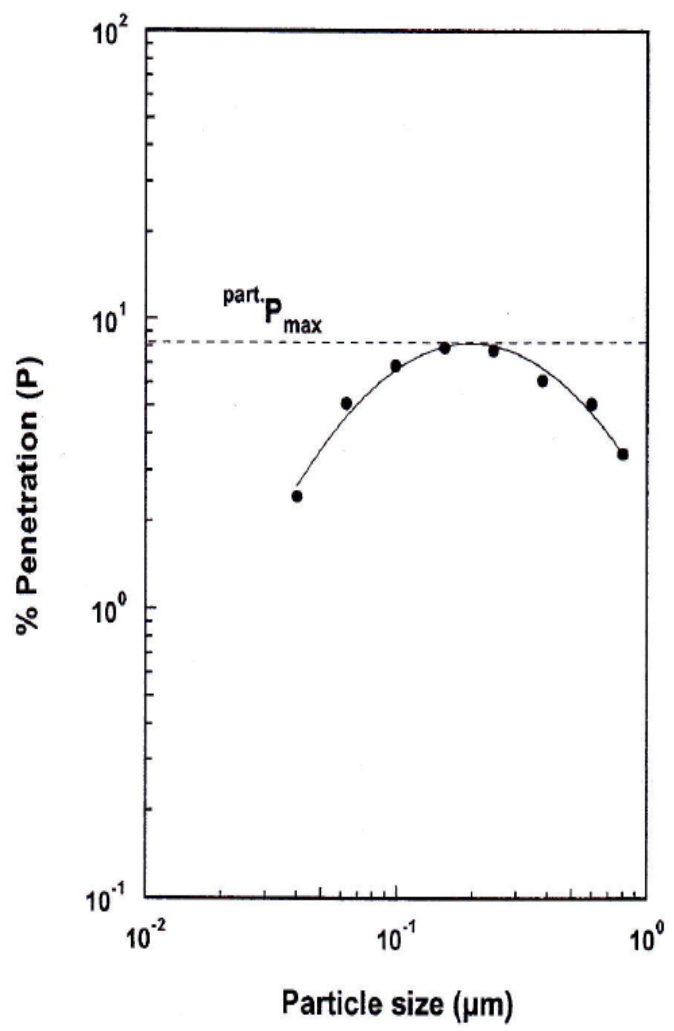

Abb.26:

Grafische Darstellung der prozentualen \% Penetration in Abhängigkeit von der Partikelgröße $(\boldsymbol{\mu m})$

Das getestete Papier (U94/40) wurde hier durch Partikel verschiedener Größe $\quad\left(10^{-2} \mu \mathrm{m} \quad\right.$ bis $\left.\quad 10^{0} \mu \mathrm{m}\right)$ beansprucht. Die Partikelpenetration wird hier im Bezug auf die Partikelgröße dargestellt.

(Sinclair und Tallentire 2002, S. 16)

Irrtümlicher Weise könnte man hieraus schlussfolgern, dass die maximale Partikelpenetration ( $\left.{ }^{\text {part. }} \mathrm{P}_{\text {max }}\right)$ eine definierte Größe für das getestete Papier (U94/40) wäre.

Vielmehr ist die maximale Partikelpenetration jedoch auch abhängig von bei der Testung durchgeführten Druckdifferenzen. Unter diesem Gesichtspunkt ergeben sich für das gleiche Papier (U94/40) bei dem gleichen Test, jedoch unter verschiedenen einwirkenden Drücken, auch unterschiedliche Werte für die Partikelpenetration.

Dies wird durch nachfolgende Darstellung deutlich. 
Abb. 27:

Darstellung der prozentualen Partikelpenetration durch ein Verpackungsmaterial (U94/40) unter der Einwirkung von 6 verschiedenen Druckwerten

Die maximale Partikelpenetration ( ${ }^{\text {part. }} \mathrm{P}_{\max }$ ) schwankt zwischen 4,5\% $\left(0,5 \mathrm{~cm} \mathrm{H}_{2} \mathrm{O}\right)$ bis zu $24 \%$ ( $20 \mathrm{~cm}$ $\left.\mathrm{H}_{2} \mathrm{O}\right)$.

(Sinclair und Tallentire 2002, S. 16)

Steigt also der Druck, so steigt auch die Partikelpenetration. Hierbei verschiebt sich jedoch der Bereich der maximalen Partikelpenetration in den Bereich kleinerer Partikelgrößen. Bei den parabelförmigen Kurvenverläufen im Diagramm zeigt sich eine Linksverschiebung.

Das bedeutet also, dass Partikel mit abnehmender Partikelgröße bei erhöhtem Druck das Papier stärker penetrieren.

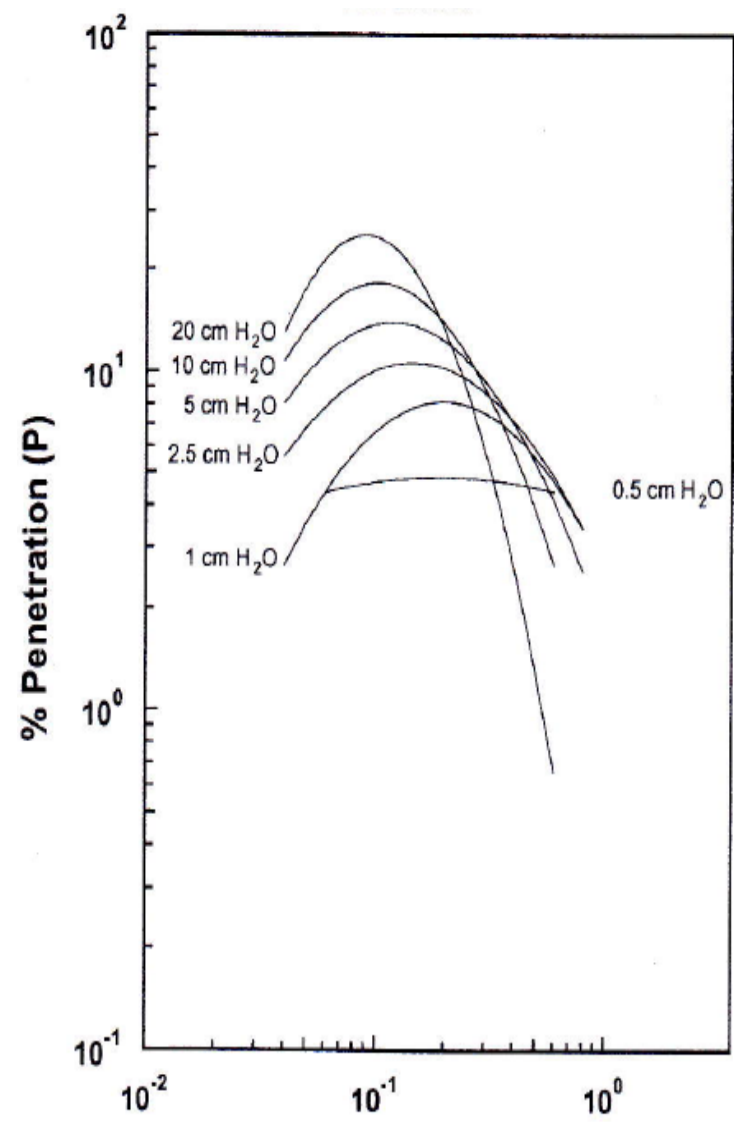

Particle size ( $\mu \mathrm{m})$

Dies stimmt mit der Funktion von Sterilisationspapier überein, denn das Papier stoppt die Partikel als eine Art Tiefenfilter.

Diese Untersuchungen zeigen demnach, dass die Filterlücke (bzw. die most penetrating particle size) keine definierte Größe darstellt, die einem ausgewählten Sterilgutverpackungspapier zugeordnet werden kann. Vielmehr variiert der Größenbereich dieser Filterlücke in Abhängigkeit von auf das Verpackungssystem einwirkenden Druckdifferenzen. Dies erfordert für die Beurteilung eines geeigneten Verpackungssystems immer auch gleichzeitig die Einschätzung der auf die Verpackung unter gegebenen Lagerungs- bzw. Transportbedingungen einwirkenden Umgebungsfaktoren (Druckschwankungen).

\subsection{Rechtlicher Anspruch im Schadensfall - Medizinproduktgesetz}

„Es ist verboten, Medizinprodukte in den Verkehr zu bringen, [...] wenn der begründete Verdacht besteht, dass sich die Sicherheit und die Gesundheit der Patienten, der Anwender oder Dritter bei sachgemäßer Anwendung, Instandhaltung und ihrer Zweckstimmung entsprechender Verwendung über ein nach den Erkenntnissen der medizinischen Wissenschaften vertretbares Maß hinausgehend unmittelbar oder mittelbar gefährden“ (MPG, 2. Abschnitt, §4, Abs.1.1, S.7).

Hier räumt jedoch die Formulierung „ über ein nach Erkenntnissen der medizinischen Wissenschaft vertretbares Maß“ dem Vertreiber der Medizinprodukte einen gewissen Argumentationsspielraum ein.

Zugleich zeigt diese Abfassung einen weiteren kritischen Punkt in der Frage der Haftbarkeit auf. 
Der Nachweis einer durch die Papierbeschaffenheit hervorgerufenen Infektion ist nahezu nicht einwandfrei zu erbringen. Dies ist zum einen darin begründet, dass eher menschliche Fehler in der Handhabung ermittelt werden können. Zum anderen spielt nicht allein das Verpackungsmaterial, sondern auch auf das Verpackungsmaterial einwirkende Faktoren (wie z.B. Druckdifferenzen) eine entscheidende Rolle im Hinblick auf eine mögliche Kontamination durch das verschlossene Verpackungssystem hindurch.

Des Weiteren führen diejenigen Keime, die im ungünstigsten Fall durch die Papierseite penetrieren können, nicht zu deutlich auffallender Symptomatik bei betroffenen Patienten, sodass ein Schadensfall nicht zwangsläufig als dieser erkannt wird. Bei der stetigen Zunahme multimorbider Patienten in der Zukunft, sollten jedoch auch diese nicht offensichtlichen Probleme in der Krankenhaushygiene an Relevanz zunehmen.

„Es ist ferner verboten, [...] wenn [...] Medizinprodukten eine Leistung beigelegt wird, die sie nicht haben“ (MPG, 2. Abschnitt, §4, Abs. 2.1, S.8). 


\section{Zusammenfassung}

Von besonderer Relevanz für die Vermeidung nosokomialer Infektionen ist die Sterilität angewandter Medizinprodukte. Hierzu zählt nicht allein der korrekte Ablauf der Sterilisation bis hin zu einer theoretischen Keimfreiheit von $10^{-6}$ Keime, sondern auch die Aufrechterhaltung der während dieses Prozesses erlangten Sterilität.

Hieraus ergibt sich die Notwendigkeit, nicht nur die Effektivität des Sterilisationsprozesses zu prüfen und geeignete validierbare Verfahrensweisen zu nutzen, sondern auch das verpackte Gut nach Lagerung unmittelbar vor der Anwendung auf seine Keimfreiheit zu testen, da es sich zu diesem Zeitpunkt in einem mikrobiologisch unkontrollierten Zustand befindet. Das Sterilitätssicherheitsniveau von 1:1.000.000 muss bis zur finalen Verwendung aufrecht erhalten werden!

Die konstruierten Testeinheiten ermöglichten es, den kompletten Sterilisationszyklus über Verpackung, Transport, Handhabung und Lagerung als gesamtes System zu überprüfen und zu bewerten. Das entwickelte Verpackungssystem ist hierbei aus zwei Komponenten aufgebaut. Im Inneren der Verpackung liegt eine Nährkartonscheibe der Papierseite zugewandt und in einer Petrischale gesichert. Diese Komponente macht den mikrobiologischen Anteil der Testeinheit aus. Hier konnte der mikrobiologische Nachweis eingedrungener Mikroorganismen erbracht werden.

Dieser mikrobiologische Anteil ist mit einem Aktivierungsanteil als zweite Komponente gekoppelt. Der Aktivierungsteil besteht im Wesentlichen aus einem Wasserreservoir, welches bei Bedarf (Aktivierung) den mikrobiologischen Anteil der Testeinheit bewässert und somit die erste Komponente, den Nährkarton, aktiviert.

Diese zwei Komponenten sind im Verpackungssystem in der Form integriert, dass eine Aktivierung des Systems nach Transport, Handhabung und Lagerung möglich war, ohne die Sterilgutverpackung öffnen zu müssen.

Die Testeinheit stellt somit ein „Double“ der im medizinischen Alltag Anwendung findenden verpackten, sterilisierten Medizinprodukte dar.

Einen ähnlichen Ansatz verfolgte bereits Widmer et al. 1992 in seinem Bestreben, einen neuen Standard im Bereich der Sterilitätstests zu etablieren, indem er forderte, alle im klinischen Alltag üblichen und auf die Verpackungen wirkenden Faktoren zu berücksichtigen.

Mit dem gleichen Gedanken wurde in dieser Arbeit eine unterschiedliche Einschätzung der wirkenden Umgebungsbedingungen in der Herzklinik Bad Rothenfelde, einer Lagerhalle für Medizinprodukte in Oldenburg und den in den Vorversuchen (2006) in Kornhäusern deponierten Testeinheiten gefunden.

Die im Rahmen der hier vorgelegten Arbeit untersuchten und getesteten Einheiten mit ihrer Papier-FolienVerbundverpackung wurden den Umgebungsbedingungen wie zum Beispiel atmosphärischem Luftdruck, Temperaturwechsel und der vorherrschenden Luftkeimzahl an den verschiedenen Standorten ausgesetzt. Weiteren Einfluss auf das Verpackungssystem stellte natürlich die Handhabung durch das Personal dar.

Während die Handhabung der Sterilgüter durch eine adäquate Ausbildung im Umgang mit Medizinprodukten vermittelt werden konnte und die Validierung von Verpackungsprozessen normativ geregelt ist, stellten die Umgebungsbedingungen im Sinne von atmosphärischem Luftdruck und Temperaturschwankungen eher schlecht abschätzbare Einflussgrößen dar. 


\section{Die Vorteile der im Rahmen dieses Feldversuches angewandten Testeinheit sind:}

- Der Keimnachweis kann in der Sterilgutverpackung erfolgen.

- Das Sterilgutverpackungssystem unterliegt Alterungsprozessen.

- Querkontaminationen bei dem Erregernachweis werden verringert.

- $\quad$ Es ist eine Anwendbarkeit im Sinne einer zusätzlichen Qualitätssicherung gegeben.

- Die Prüfung der Barrierewirksamkeit selbst stellt kein nennenswertes Rekontaminationsrisiko dar.

Die Gesamtheit der in Oldenburg und in Bad Rothenfelde gelagerten Testeinheiten erwies sich nach Lagerung, Transport, Handhabung, Aktivierung und Anzüchtung möglicher eingedrungener Keime als steril.

Anders war dies jedoch im Rahmen der 2006 im Institut für Hygiene und Umweltmedizin durchgeführten Vorversuche in den Kornhäusern Dransfeld, Scharzfeld und Obernjesa. Hier wurden Kontaminationsraten der verpackten Testsysteme von 55,2 \% bis hin zu 84,2 \% nachgewiesen.

Anhand dieser Ergebnisse konnte direkt aufgezeigt werden, dass die Wahrung der Sterilität nicht allein von der Sterilgutverpackung abhängig ist, sondern bei regelrechter Anwendung maßgeblich von vorherrschenden Umgebungsbedingungen beeinträchtigt wird und vor allem durch Druckgradienten durchbrochen werden kann.

Es kann grundsätzlich gesagt werden, dass vor allem der Vorversuch in den Kornhäusern eindrucksvoll zeigt, dass der Effektivität der Sterilbarrierefunktion von Papier-Folien-Verbundverpackungen in Bezug auf die Aufrechterhaltung der Sterilität deutliche Grenzen gesetzt sind.

Auch wenn solch ein deutlich mangelhaftes Ergebnis unter den getesteten realitätsnahen Bedingungen der klinischen Versorgung mit Sterilgütern nicht bestätigt wurde, muss jedoch die Problematik der Rekontamination auch hier weiterhin diskutiert werden. Anhand der ermittelten Werte konnten die mikrobiologische Beanspruchung, die Filtereffektivität und der logarithmische Reduktionsfaktor für das Verpackungsmaterial an den verschiedenen Lagerungsstandorten berechnet werden.

Die in diesem Feldversuch eingesetzten Testeinheiten können kommerziell weiterentwickelt werden, um dem Anwender ein Hilfsmittel an die Hand zu geben, welches die Sterilität seiner Produkte und somit deren einwandfreie Handhabung im Praxisalltag belegen bzw. unmittelbar vor dem Gebrauch überprüfen kann. Der Nutzer kann so einen Eindruck von dem aktuellen Zustand seiner in Betrieb befindlichen Sterilprodukte erhalten und das Qualitätsmanagement in seiner Praxis steigern. Finden die Testeinheiten in diesem Sinne Anwendung, kann der Anbieter nach Auswertung der Testeinheiten eine Analyse des gelagerten oder transportierten Sterilgutes erstellen. Anhand resultierender Testergebnisse kann daraufhin eine Empfehlung seitens des Anbieters der Testeinheiten getroffen werden, welche dem Anwender zur Optimierung des Managements oder der Handhabung von verpacktem Sterilgut hilft. Somit kann das sterilisierte Medizinprodukt direkt vor der Verwendung auf seinen mikrobiologischen Zustand überprüft werden.

Es ist somit möglich ein validierbares, rekontaminationsfreies, sicheres und reproduzierbares Verfahren zum Einsatz zu bringen. Die daraus resultierende Sicherheit in der Sterilgutversorgung kommt der Primärprävention nosokomialer Infektionen zu Gute.

Mit Hilfe dieser Arbeit konnte die Weiterentwicklung der bereits durch Dunkelberg und Fleitmann- Glende im Expositionskammerverfahren getesteten Methoden erfolgen. Die Eignung der Testeinheit konnte auf diesem Weg auch unter realitätsnahen Bedingungen des klinischen Betriebs aufgezeigt werden. Möglichkeiten der Ergebnisanalyse, wie die Bestimmung des logarithmischen Reduktionsfaktors, der Filtereffektivität oder der mikrobiologischen Beanspruchung können dem Anwender dieses Testsystems zur Verfügung gestellt werden. 


\section{Anhang}

\subsection{Abkürzungsverzeichnis}

\begin{tabular}{|c|c|}
\hline Abkürzung & Ausgeschriebene Form, Bedeutung \\
\hline 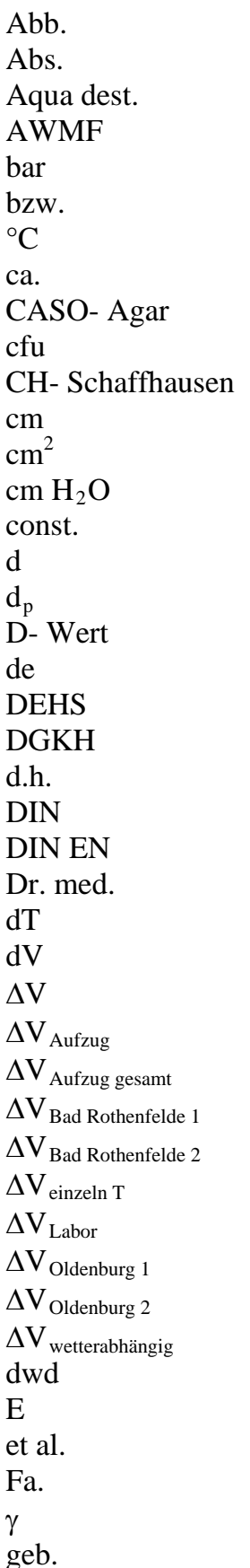 & $\begin{array}{l}\text { Abbildung } \\
\text { Absatz } \\
\text { destilliertes Wasser } \\
\text { Arbeitsgemeinschaft der Wissenschaftlichen Medizinischen Fachgesellschaften } \\
\text { bar } \\
\text { beziehungsweise } \\
\text { Grad Celsius } \\
\text { circa } \\
\text { Casein- Sojalecithin- Agar (mit Pepton aus Casein und Soja) } \\
\text { colony forming unit } \\
\text { Confoederatio Helvetica- Schaffhausen } \\
\text { Zentimeter } \\
\text { Quadratzentimeter } \\
\text { Zentimeter Wassersäule } \\
\text { konstant } \\
\text { Durchmesser } \\
\text { Partikeldurchmesser } \\
\text { Dezimalreduktionswert (siehe Seite 6) } \\
\text { ISO 3166-1 alpha-2 Code für Deutschland } \\
\text { Prüfaerosol unbelebter Partikel - Di- Ethyl- Hexyl- Sebacat } \\
\text { Deutsche Gesellschaft für Krankenhaushygiene e.V. } \\
\text { das heißt } \\
\text { Deutsches Institut für Normung e.V. } \\
\text { in nationalen Standard überführte (übersetzte) Europa- Normen } \\
\text { Doctor medicinae (Doktor der Medizin) } \\
\text { Temperaturdifferenz } \\
\text { Volumendifferenz } \\
\text { Volumendifferenz } \Delta \mathrm{V} \\
\text { Volumendifferenz } \Delta \mathrm{V} \text { (durch eine Aufzugsfahrt bedingte Druckwechsel) } \\
\text { Volumendifferenz } \Delta \mathrm{V} \text { (durch alle Aufzugsfahrten bedingte Druckwechsel) } \\
\text { Volumendifferenz } \Delta \mathrm{V} \text { Bad Rothenfelde } 1 \text { (durch Druckdifferenzen) } \\
\text { Volumendifferenz } \Delta \mathrm{V} \text { Bad Rothenfelde } 2 \text { (durch Temperaturdifferenzen) } \\
\text { Volumendifferenz } \Delta \mathrm{V} \text { durch Temperaturschwankungen pro Testeinheit (in ml) } \\
\text { Volumendifferenz } \Delta \mathrm{V} \text { Kontrollgruppe } 2 \\
\text { Volumendifferenz } \Delta V \text { Oldenburg } 1 \text { (durch Druckdifferenzen) } \\
\text { Volumendifferenz } \Delta \mathrm{V} \text { Oldenburg } 2 \text { (durch Temperaturdifferenzen) } \\
\text { Volumendifferenz } \Delta \mathrm{V} \text { (durch wetterabhängige atmosphärische Druckwechsel) } \\
\text { Deutscher Wetterdienst } \\
\text { Fraktionsabscheidegrad } \\
\text { et alii (und andere) } \\
\text { Firma } \\
\text { Gamma - Volumenausdehnungskoeffizient (konstant: 0,0036/K) } \\
\text { geborene (zur Angabe des Geburtsnamens) }\end{array}$ \\
\hline
\end{tabular}




\begin{tabular}{|c|c|}
\hline 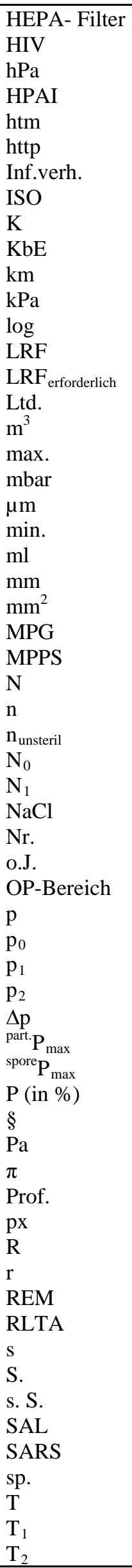 & $\begin{array}{l}\text { High Efficiency Particulate Airfilter } \\
\text { Human Immundeficiency Virus } \\
\text { Hektopascal } \\
\text { Highly Pathogenic Avian Influenza } \\
\text { Hyper Text Markup } \\
\text { Hyper Text Transfer Protocol } \\
\text { Infektionsverhütung } \\
\text { International Standard Organisation } \\
\text { Kelvin } \\
\text { Koloniebildende Einheiten } \\
\text { Kilometer } \\
\text { Kilopascal } \\
\text { Logarithmus } \\
\text { Logarithmischer Reduktionsfaktor (siehe Seite 37) } \\
\text { erforderlicher LRF, um das Sterilgut steril zu wahren } \\
\text { Limited (Gesellschaftsform im anglo- amerikanischen Sprachraum) } \\
\text { Kubikmeter } \\
\text { maximal } \\
\text { Millibar } \\
\text { Mikrometer } \\
\text { minimal } \\
\text { Milliliter } \\
\text { Millimeter } \\
\text { Quadratmillimeter } \\
\text { Medizinproduktgesetz } \\
\text { most penetrating particle size (siehe Seite 82) } \\
\text { Anzahl (Aufzugsfahrten) } \\
\text { Anzahl (Testeinheiten) } \\
\text { Anzahl unsteriler Testeinheiten } \\
\text { mikrobiologische Beanspruchung } \\
\text { Rekontamination der in der Verpackung enthaltenen Nährkartonscheibe } \\
\text { Natriumchlorid } \\
\text { Nummer } \\
\text { ohne Jahresangabe } \\
\text { Operationsbereich } \\
\text { Druck } \\
\text { mittlerer atmosphärischer Luftdruck } \\
\text { Ausgangswert Druck } \\
\text { Endwert Druck } \\
\text { Druckdifferenz } \\
\text { maximale Partikelpenetration } \\
\text { maximale Sporenpenetration } \\
\text { prozentuale Penetration/ Fraktionsdurchlassgrad } \\
\text { Paragraf } \\
\text { Pascal } \\
\text { griechischer Buchstabe Pi - Kreiszahl } \pi=3,14159 . . . \\
\text { Professor } \\
\text { 1px } \text { min.96* 10-8m (für diese Grafik bestimmter Maßstab, siehe Seite 79) } \\
\text { Gaskonstante } \\
\text { Radius } \\
\text { Rasterelektronenmikroskop } \\
\text { Raumlufttechnische Anlage } \\
\text { Sekunde } \\
\text { Seite } \\
\text { siehe Seite } \\
\text { sterility assurance level - Sterilitätssicherheitswert (siehe Seite 40) } \\
\text { Severe Acute Respiratory Syndrome } \\
\text { species } \\
\text { Temperatur } \\
\text { maximaler Tagestemperaturwert } \\
\end{array}$ \\
\hline
\end{tabular}




\begin{tabular}{|l|l|}
\hline toxikol. & toxikologisch \\
u.a. & unter anderem \\
$\mathrm{URL}$ & Uniform Resource Locator (Link zu einer Internetseite) \\
$\mathrm{V}$ & Volumen \\
$\mathrm{V}_{1}$ & Ausgangsvolumen \\
$\mathrm{V}_{2}$ & Endvolumen \\
$\mathrm{V}^{\circ}$ gesamt & Gesamtvolumeneinstrom durch die Summe aller wirkenden Druckwechsel \\
vgl. & vergleiche \\
VWR Darmstadt & Van Waters and Rogers Incorporated Company (Chemie- Vertrieb) \\
www & World Wide Web \\
z.B. & zum Beispiel \\
z- Wert & Keimspezifischer Wert - zusätzliche Temperatur (siehe Seite 6) \\
& \\
\hline
\end{tabular}




\subsection{Literaturverzeichnis}

Augustowska M, Dutkiewicz J (2006):

Variability of airborne microflora in a hospital ward within a period of one year

Ann Agric Environ Med 13 (1), 99-106

AWMF online

Arbeitsgemeinschaft der Wissenschaftlichen Medizinischen Fachgesellschaften

Leitlinien zur Hygiene in Klinik und Praxis - Raumlufttechnische Anlagen (RLTA)

URL

http://www.uni-duesseldorf.de.awmf/ll/029-020.htm

[Eingesehen am 23.09.2010; 15:32 Uhr]

Bergmair J, Washüttl M, Wepner B:

Farbstofftest (Rhodamin B- Test) ;

in: Prüfpraxis für Kunststoffverpackungen: Lebensmittel- , Pharma- und Kosmetikverpackungen;

hrsg. v. Bergmair J; Behr`s Verlag DE 2004, 249

Bundesministerium für Verkehr, Bau und Stadtentwicklung

Deutscher Wetterdienst

Tageswerte für Bremen (Oldenburg)

URL

http://www.dwd.de/bvbw/appmanager/bvbw/dwdwwwDesktop?_nfpb=true\&_pageLabel=_dwdwww_klima_um welt_klimadaten_deutschland\&T82002gsbDocumentPath=Navigation\%2FOeffentlichkeit\%2FKlima_Umwelt \%2FKlimadaten\%2Fkldaten_kostenfrei\%2Fausgabe_tageswerte_node.html\%3F_nnn\%3Dtrue

Download: produkt_klima_Tageswerte18900101_20111231_00691.txt

[Eingesehen am 14.04.2012; 16:14 Uhr]

Bundesministerium für Verkehr, Bau und Stadtentwicklung

Deutscher Wetterdienst

Tageswerte für Münster/ Osnabrück (Bad Rothenfelde)

URL

http://www.dwd.de/bvbw/appmanager/bvbw/dwdwwwDesktop?_nfpb=true\&_pageLabel=_dwdwww_klima_um welt_klimadaten_deutschland\&T82002gsbDocumentPath=Navigation\%2FOeffentlichkeit\%2FKlima_Umwelt \%2FKlimadaten\%2Fkldaten_kostenfrei\%2Fausgabe_tageswerte_node.html\%3F_nnn\%3Dtrue Download: produkt_klima_Tageswerte19891001_20111231_01766.txt

[Eingesehen am 14.04.2012; 16:50 Uhr]

Cooper MS (1982):

The evolving USP sterility test

J Percent Sci Technol 36, 256-259

DIN 58953-7 Sterilisation - Sterilgutversorgung - Teil 7: Anwendungstechnik von Sterilisationspapier, Vliesstoffen, gewebten textilen Materialien, Papierbeuteln und siegelfähigen Klarsichtbeuteln und -schläuchen Deutsches Institut für Normung e. V.:

DIN 58953-7; Beuth- Verlag, Berlin 2010

DIN 58953-8 Sterilisation - Sterilgutversorgung - Teil 8: Logistik von sterilen Medizinprodukten

Deutsches Institut für Normung e. V.:

DIN 58953-8; Beuth- Verlag, Berlin 2010

DIN 58953-9 Sterilisation - Sterilgutversorgung - Teil 9: Anwendungstechnik von Sterilisierbehältern

Deutsches Institut für Normung e. V.:

DIN 58953-9; Beuth- Verlag, Berlin 2010

DIN EN 285 + A2 Sterilisation - Dampf- Sterilisatoren - Groß- Sterilisatoren

Deutsches Institut für Normung e. V.:

DIN EN 285 + A2; Beuth- Verlag, Berlin 2006 + 2009 
DIN EN 556-1 Sterilisation von Medizinprodukten -Anforderungen an Medizinprodukte, die als „STERIL“ gekennzeichnet werden; Teil 1: Anforderungen an Medizinprodukte, die in der Endverpackung sterilisiert wurden

Deutsches Institut für Normung e. V.:

DIN EN 556-1; Beuth- Verlag, Berlin 2001

DIN EN 868-3 Verpackungsmaterialien und -systeme für zu sterilisierende Medizinprodukte - Teil 3: Papier zur Herstellung von Papierbeuteln (festgelegt in EN 868-4) und zur Herstellung von Klarsichtbeuteln und -schläuchen (festgelegt in EN 868-5) - Anforderungen und Prüfverfahren

Deutsches Institut für Normung e. V.:

DIN EN 868-3; Beuth- Verlag, Berlin 1999

DIN EN 868-3 Verpackungen für in der Endverpackung zu sterilisierende Medizinprodukte - Teil 3: Papier zur Herstellung von Papierbeuteln (festgelegt in EN 868-4) und zur Herstellung von Klarsichtbeuteln und -schläuchen (festgelegt in EN 868-5) - Anforderungen und Prüfverfahren

Deutsches Institut für Normung e. V.:

DIN EN 868-3; Beuth- Verlag, Berlin 2009

DIN EN 868-7 Verpackungen für in der Endverpackung zu sterilisierende Medizinprodukte - Teil 7: Klebemittelbeschichtetes Papier für Niedertemperatur- Sterilisationsverfahren - Anforderungen und Prüfverfahren

Deutsches Institut für Normung e. V.:

DIN EN 868-7; Beuth- Verlag, Berlin 2009

DIN EN 1822-1 Schwebstofffilter (EPA, HEPA und ULPA) - Teil 1: Klassifikation, Leistungsprüfung, Kennzeichnung

Deutsches Institut für Normung e.V.:

DIN EN 1822-1; Beuth- Verlag, Berlin 2009

DIN EN 1822-3 Schwebstofffilter (EPA, HEPA und ULPA) - Teil 3: Prüfung des planen Filtermediums

Deutsches Institut für Normung e.V.:

DIN EN 1822-3; Beuth- Verlag, Berlin 2009

DIN EN 1822-4 Schwebstofffilter (EPA, HEPA und ULPA) - Teil 3: Leckprüfung des Filterelementes (ScanVerfahren)

Deutsches Institut für Normung e.V.:

DIN EN 1822-4; Beuth- Verlag, Berlin 2009

DIN EN ISO 11607-1 Verpackungen für in der Endverpackung zu sterilisierende Medizinprodukte Teil 1: Anforderungen an Materialien, Sterilbarrieresysteme und Verpackungssysteme Deutsches Institut für Normung e.V.:

DIN EN ISO 11607-1; Beuth- Verlag, Berlin 2009

DIN EN ISO 11607-2 Verpackungen für in der Endverpackung zu sterilisierende Medizinprodukte - Teil 2:

Validierungsanforderungen an Prozesse der Formgebung, Siegelung und des Zusammenstellens

Deutsches Institut für Normung e. V.:

DIN EN ISO 11607-2; Beuth- Verlag, Berlin 2006

DIN EN ISO 17665-1 Sterilisation von Produkten für die Gesundheitsfürsorge - Feuchte Hitze - Teil 1: Anforderungen an die Entwicklung, Validierung und Lenkung der Anwendung eines Sterilisationsverfahrens für Medizinprodukte

Deutsches Institut für Normung e. V.:

DIN EN ISO 17665-1; Beuth- Verlag, Berlin 2006

Dunkelberg H, Fleitmann-Glende F (2006):

Measurement of the microbial barrier effectiveness of sterilization containers in terms of the log reduction value for prevention of nosocomial infections Am J Infect Control 34 (5), 285-289 
Dunkelberg H, Schmelz U:

Entwicklung eines Testkits zur Überwachung der Barriereeigenschaften von Sterilgutverpackungssystemen im Krankenhausroutinebetrieb

Erste Ergebnisse

(Vortrag gehalten 23. 05. 2007)

in: 19. DOSCH- Symposium (Österreichische Gesellschaft für Hygiene, Mikrobiologie und Präventivmedizin), Millstatt/ Kärnten, 21. - 23. Mai 2007; o. Hrsg., o. Verl., o. O. 2007

Dunkelberg H, Schmelz U:

Aufrechterhaltung der Sterilität während Lagerung und Transport;

in: Praxis der Sterilisation, Desinfektion, Konservierung;

hrsg. v. Wallhäuser KH, Thieme Verlag Stuttgart, New York 2008a, 132

modifiziert nach Dunkelberg H, Schmelz U:

Testkit zur routinemäßigen Überwachung der Barriereeigenschaften von Sterilgutverpackungssystemen unter Praxisbedingungen.

in: Poster im Rahmen des DGKH- Kongresses, Berlin 20. - 23. April 2008; o. Hrsg., o. Verl., o. O. 2008b

Dunkelberg H, Schmelz U (o. J.)

Universitätsmedizin Göttingen

Sterilgut auf höchstem mikrobiologischen Qualitätsniveau verpacken, transportieren und lagern

URL

http://www.sterilgutverpackung-bewerten.de/5e65d9c1a46cab8c47ea8d4cd33679d2_Infomaterial_Haupttext.pdf [Eingesehen am 13.04.2012; 14:23 Uhr]

Dunkelberg H, Wedekind S (2002):

A new method for testing the effectiveness of the microbial barrier properties of packaging materials for sterile products. (Eine neue Methode zur Wirksamkeitsprüfung von Sterilgutverpackungen in der Praxis)

Biomed Technik (Berlin) 47, 290-293

Dunkelberg H, Wedekind S (2004):

Preliminary results for a new final package test to assess the quality of sterile package systems

Infect Control Hosp Epidemiol 25 (1), 26-29

Eidgenössische Materialprüfungs- und Forschungsanstalt (2004)

Im Kampf gegen tückische Keime

URL

http://www.empa.ch/plugin/template/empa/*/27408/---/l=1

[Eingesehen am 21.06.2011; 21:08 Uhr]

Harte JA, Miller CH (2004):

Sterilization update 2003

Compend Contin Educ Dent 25 (1), 24-29

Kahn MA (1983):

Invasion von Vorratsschädlingen durch Verschlüsse

Anz. Schädlingskde., Pflanzenschutz, Umweltschutz 56, 91-94

Kelsey JC (1972):

The myth of surgical sterility

The Lancet 1972, II, 1301-1303

Klapes NA, Greene VW, Langholz AC (1987a)

Microbial contamination associated with routine aseptic practice

Hosp Infect (1987); 10: 299-304

Klapes NA, Greene VW, Langholz AC, Hunstiger C (1987b)

Effect of long- term storage on sterile status of devices in surgical packs

Am J Infect Control (1987); 8 (7): 289-293 
Krysińska- Traczyk E, Pande BN, Skórska C, Sitkowska J, Prażmo Z, Cholewa G, Dutkiewicz J (2005): Exposure of Indian agricultural workers to airborne microorganisms , dust and endotoxin during handling of various plant products

Ann Agric Environ Med 12, 269-275

Luftfilter- Prüfverfahren zur Prüfung von Schwebstofffiltern

(CTROX Filter GmbH 2009

URL

http://www.trox.de/xpool/download/de/technical_documents/filters/leaflets/P_2_EN1822.pdf

[Eingesehen am 05.09.2012; 18:10 Uhr]

Medipack 2006

Mögliche serienfertigungsfähige Halterung für die beiden Komponenten der Testeinheit (im Rahmen der Entwicklung der Testeinheit in Auftrag gegeben)

Entwurf: Medipack, CH- Schaffhausen, 2006

Medizinproduktgesetz (MPG) - Bundesministerium der Justiz (2010)

Verbote zum Schutz von Patienten, Anwendern und Dritten

MPG, 2. Abschnitt, §4, Absatz 1.1, S.7

[Stand: 24.07.2010]

Medizinproduktgesetz (MPG) - Bundesministerium der Justiz (2010)

Verbote zum Schutz von Patienten, Anwendern und Dritten

MPG, 2. Abschnitt, §4, Absatz 2.1, S.8

[Stand: 24.07.2010]

Metzger S (o. J.)

Brown'sche Bewegung

URL

http://schulen.eduhi.at/riedgym/physik/10/waerme/temperatur/brownsche_bewegung.htm)

[Eingesehen am 18.07.2010; 15:14 Uhr]

Moggio M, Goldner JL, McCollum DE, Beissinger SF (1979):

Wound infections in patients undergoing total hip arthroplasty.

Ultraviolet light for the control of airborne bacteria.

Arch Surg 114 (7), 815 - 823

Motorlexikon

Wirkungsweise von Partikelfiltern

URL

http://www.motorlexikon.de/?I=5441

[Eingesehen am 12.03.2011; 19:34 Uhr]

Odlaug TE, Ocwieja DA, Purohit KS, Riley RM, Young WE (1984):

Sterility assurance for terminally sterilized products without end- product sterility testing

J Percent Sci Technol 38, 141-147

Otani Y, Eryn K, Furuuchi M, Tajima N, Tekasakul P (2007):

Inertial Classification of Nanoparticles with Fibrous Filters

Aerosol Air Quality Res 7 (3), 343-352

redam- instrumente $\mathrm{GmbH}$ - chirurgie/medizintechnik/containersysteme

URL

http://www.redam-instrumente.de/container-siebkoerbe.html

[Eingesehen am 24.05.2010; 16:22 Uhr]

Schenke S:

Erfassung und Bewertung von mikrobiellen volatilen organischen Substanzen (MVOC) in schimmelpilzfreien Innenräumen im Rahmen der Giessener Innenraumallergen Studie (GINA- STUDIE)

Med. Diss. Justus- Liebig- Universität Gießen 2009, 16 
Schimmel - Schimmelpilze - Schimmelpilzgifte

Rhizopus - Rhizopus stolonifer im REM bei 1250 facher Vergrößerung

URL

http://www.schimmel-schimmelpilze.de/rhizopus.html

[Eingesehen am 13.03.2011; 16:07 Uhr]

Schwartz R, Davis R (1990):

Safe storage times for sterile dental packs

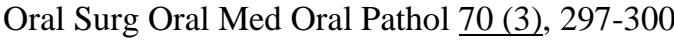

Sinclair CS, Tallentire A (2002):

Definition of a correlation between microbiological and physical particulate barrier performances for porous medical packaging materials

PDA J Pharm Sci Technol 트, 11-19

Swan JRM, Crook B (1998):

Airborne microorganisms associated with grain handling

Ann Agric Environ Med 5, 7-15

Szabo E:

Experimentelle Untersuchungen luftgetragener Partikel und Schimmelpilze in Pferdeställen

Diss. med. vet. Freie Universität Berlin 2007, 133-134

Tham KW, Zuraimi MS (2005):

Size relationship between airborne viable bacteria and particles in a controlled indoor environment study. Indoor Air 15 (9), 48-57

Thomas P, Matuschek H (2005)

Brownsche Molekularbewegung T8 - Grafische Darstellung der Bewegung

URL

http://physik.spdj.de/PhHa_T8.pdf

[Eingesehen am 21.06.2011; 21:51 Uhr]

Webster J, Lloyd W, Ho P, Burridge C, George N (2003):

Rethinking sterilization practices: Evidence for event- related outdating

Infect Control Hosp Epidemiol 24, 622-624

Wetterstation Göttingen - Monatsanalyse Juni 2006

URL

www.wetterstation-goettingen.de/analysen/monat/062006.pdf

[Eingesehen am 13.04.2012]

Wetterstation Göttingen - Monatsanalyse Juli 2006

URL

www.wetterstation-goettingen.de/analysen/monat/072006.pdf

[Eingesehen am 13.04.2012]

Wetterstation Göttingen - Monatsanalyse August 2006

URL

www.wetterstation-goettingen.de/analysen/monat/082006.pdf

[Eingesehen am 13.04.2012]

Wetterstation Göttingen - Monatsanalyse September 2006

URL

www.wetterstation-goettingen.de/analysen/monat/092006.pdf

[Eingesehen am 13.04.2012]

Wetterstation Göttingen - Monatsanalyse Oktober 2006

URL

www.wetterstation-goettingen.de/analysen/monat/102006.pdf

[Eingesehen am 13.04.2012] 
Wetterstation Göttingen - Monatsanalyse November 2006

URL

www.wetterstation-goettingen.de/analysen/monat/112006.pdf

[Eingesehen am 13.04.2012]

Wetterstation Göttingen - Monatsanalyse Dezember 2006

URL

www.wetterstation-goettingen.de/analysen/monat/122006.pdf

[Eingesehen am 13.04.2012]

Wetterstation Göttingen - Monatsanalyse März 2008

URL

www.wetterstation-goettingen.de/analysen/monat/032008.pdf

[Eingesehen am 13.04.2012]

Wetterstation Göttingen - Monatsanalyse April 2008

URL

www.wetterstation-goettingen.de/analysen/monat/042008.pdf

[Eingesehen am 13.04.2012]

Wetterstation Göttingen - Monatsanalyse Mai 2008

URL

www.wetterstation-goettingen.de/analysen/monat/052008.pdf

[Eingesehen am 13.04.2012]

Widmer AF, Houston A, Bollinger E, Wenzel RP (1992):

A new standard for sterility testing for autoclaved surgical trays

Hosp Infect 21, 253-260

Wolf C (2003):

Qualitätsmanagement im Sterilgutverpackungsprozess - Verpackungssystem, Validierung, Lagerung Krh.- Hyg. + Inf. verh. 25 (6), 254 


\subsubsection{Abbildungsverzeichnis}

Abbildung 1:

Modifizierte Darstellung der Fraktionsabscheidegradkurve aus DIN EN 1822-3

Seite 10

Quelle:

DIN EN 1822-3 Schwebstofffilter (EPA, HEPA und ULPA) - Teil 3: Prüfung des planen Filtermediums

Deutsches Institut für Normung e.V. :

DIN EN 1822-3; Beuth- Verlag, Berlin 2009, 21

Abbildung 2:

Querschnitt durch eine Testeinheit mit darin befindlicher, präparierter Petrischale

Seite 22

Quelle:

modifiziert nach Dunkelberg H, Schmelz U:

Testkit zur routinemäßigen Überwachung der Barriereeigenschaften von Sterilgutverpackungssystemen unter Praxisbedingungen.

in: Poster im Rahmen des DGKH- Kongresses, Berlin 20. - 23. April 2008; o. Hrsg., o. Verl., o. O. 2008b

Abbildung 3:

Ansicht einer präparierten Petrischale als mikrobiologischer Teil der Testeinheit

Seite 22

Abbildung 4:

Darstellung der erforderlichen Komponenten zur Aufnahme des Wasserreservoirs der Testeinheit

Seite 23

Abbildung 5:

Schematische Abbildung der gesamten Testeinheit im Querschnitt

Seite 24

Quelle:

modifiziert nach Dunkelberg H, Schmelz U:

Testkit zur routinemäßigen Überwachung der Barriereeigenschaften von Sterilgutverpackungssystemen unter Praxisbedingungen.

in: Poster im Rahmen des DGKH- Kongresses, Berlin 20. - 23. April 2008; o. Hrsg., o. Verl., o. O. 2008b

Abbildung 6:

Testeinheit in zu prüfender Sterilgutverpackung, fertig zur Verwendung

Seite 24

Abbildung 7:

Anteil unsteriler Testeinheiten in Abhängigkeit vom Expositionsort und der gesamten mikrobiologischen

Beanspruchung $\mathrm{N}_{0}$

Seite 45

Abbildung 8:

Gesamte mikrobiologische Beanspruchung $\mathrm{N}_{0}$ und prozentualer Anteil unsteriler Testsysteme in Abhängigkeit vom Lagerungsort

Seite 57

Abbildung 9:

Mögliche serienfertigungsfähige Halterung für die beiden Komponenten der Testeinheit

Seite 67

Medipack 2006

Mögliche serienfertigungsfähige Halterung für die beiden Komponenten der Testeinheit

(im Rahmen der Entwicklung der Testeinheit in Auftrag gegeben)

Entwurf: Medipack, CH- Schaffhausen, 2006 
Abbildung 10:

Schematische Darstellung des Verhältnisses der Flächeninhalte der gesamten Innenfläche zur Fläche der Nährkartonscheibe

Seite 68

Abbildung 11:

Graphische Darstellung des Flächenverhältnisses zwischen Nährkartonscheibe und der gesamten Innenfläche der Verpackung

Seite 69

Abbildung 12:

Verbesserungsentwurf der Testeinheit

Seite 70

Abbildung 13:

Schweißsiegelnahttest mit Rhodamin-B-Lösung, eine visuell nicht zu erkennende Leckage wird detektiert Seite 71

Abbildung 14:

Darstellung der Bestandteile eines Sterilgutcontainers

Seite 74

Quelle:

redam- instrumente GmbH - chirurgie/medizintechnik/containersysteme

URL

http://www.redam-instrumente.de/container-siebkoerbe.html

[Eingesehen am 24.05.2010; 16:22 Uhr]

Abbildung 15:

Brownsche Molekularbewegung - Schematische Darstellung des random walk

Seite 77

Quelle:

Metzger S (o. J.)

Brown'sche Bewegung

URL

http://schulen.eduhi.at/riedgym/physik/10/waerme/temperatur/brownsche_bewegung.htm)

[Eingesehen am 18.07.2010; 15:14 Uhr]

Abbildung 16:

Grafische Darstellung der Bewegung

Thomas P, Matuschek H (2005)

Brownsche Molekularbewegung T8 - Grafische Darstellung der Bewegung

Seite 77

Quelle:

URL

http://physik.spdj.de/PhHa_T8.pdf

[Eingesehen am 21.06.2011; 21:51 Uhr]

Abbildung 17:

Darstellung der physikalischen Phänomene eines Papierfilters anhand einer einzelnen Faser

Seite 78

Quelle:

Motorlexikon

Wirkungsweise von Partikelfiltern

URL

http://www.motorlexikon.de/?I=5441

[Eingesehen am 12.03.2011; 19:34 Uhr] 
Abbildung 18:

Graphische Darstellung der Filterlücke

Seite 78

Quelle:

Motorlexikon

Wirkungsweise von Partikelfiltern

URL

http://www.motorlexikon.de/?I=5441

[Eingesehen am 12.03.2011; 19:34 Uhr]

Abbildung 19:

Cladosporium sp.

Seite 79

Quelle:

Szabo E:

Experimentelle Untersuchungen luftgetragener Partikel und Schimmelpilze in Pferdeställen

Diss. med. vet. Freie Universität Berlin 2007, 133

Abbildung 20:

Rhizopus sp.

Seite 79

Quelle:

Schimmel - Schimmelpilze - Schimmelpilzgifte

Rhizopus - Rhizopus stolonifer im REM bei 1250 facher Vergrößerung

URL

http://www.schimmel-schimmelpilze.de/rhizopus.html

[Eingesehen am 13.03.2011; 16:07 Uhr]

Abbildung 21:

Penicillium sp.

Seite 79

Quelle:

Szabo E:

Experimentelle Untersuchungen luftgetragener Partikel und Schimmelpilze in Pferdeställen

Diss. med. vet. Freie Universität Berlin 2007, 134

Abbildung 22:

Darstellung des Fraktionsabscheidegrades

Seite 80

Quelle:

Luftfilter- Prüfverfahren zur Prüfung von Schwebstofffiltern

(C)TROX Filter GmbH 2009

URL

http://www.trox.de/xpool/download/de/technical_documents/filters/leaflets/P_2_EN1822.pdf

[Eingesehen am 05.09.2012; 18:10 Uhr]

Abbildung 23:

Funktion des Partikeldurchmessers $d_{p}$ für zwei verschiedene Filtermediumsgeschwindigkeiten

Seite 81

Quelle:

DIN EN 1822-1 Schwebstofffilter (EPA, HEPA und ULPA) - Teil 1: Klassifikation, Leistungsprüfung, Kennzeichnung

Deutsches Institut für Normung e.V.:

DIN EN 1822-1; Beuth- Verlag, Berlin 2009, 14

Abbildung 24:

Das Prinzip eines Trägheitsfilters

Seite 82

Quelle:

Otani Y, Eryn K, Furuuchi M, Tajima N, Tekasakul P (2007):

Inertial Classification of Nanoparticles with Fibrous Filters

Aerosol Air Quality Res 7 (3), 343-352 
Abbildung 25:

Grafische Darstellung der prozentualen \% Sporenpenetration in Abhängigkeit von der Durchflussrate sporenbeladener Luft

Seite 82

Quelle:

Sinclair CS, Tallentire A (2002):

Definition of a correlation between microbiological and physical particulate barrier performances for porous medical packaging materials

PDA J Pharm Sci Technol 트, 15

Abbildung 26:

Grafische Darstellung der prozentualen \% Penetration in Abhängigkeit von der Partikelgröße $(\mu \mathrm{m})$

Seite 83

Quelle:

Sinclair CS, Tallentire A (2002):

Definition of a correlation between microbiological and physical particulate barrier performances for porous medical packaging materials

PDA J Pharm Sci Technol ㅌ6, 16

Abbildung 27:

Darstellung der prozentualen Partikelpenetration durch ein Verpackungsmaterial (U94/40) unter der Einwirkung von 6 verschiedenen Druckwerten

Seite 84

Quelle:

Sinclair CS, Tallentire A (2002):

Definition of a correlation between microbiological and physical particulate barrier performances for porous medical packaging materials

PDA J Pharm Sci Technol 트, 16 


\subsubsection{Tabellenverzeichnis}

Tabelle 1:

Im Vorversuch 2006 ermittelte Rohdaten

Seite 18

Tabelle 2:

Die in der Luft eines Operationssaales nachgewiesenen Keimarten (grobe Zuordnung)

Seite 27

Quelle:

Moggio M, Goldner JL, McCollum DE, Beissinger SF (1979):

Wound infections in patients undergoing total hip arthroplasty.

Ultraviolet light for the control of airborne bacteria.

Arch Surg 114 (7), 815 - 823

\section{Tabelle 3:}

Lagerungsorte und -zeiten der Testeinheiten im Überblick

Seite 30

Tabelle 4:

Schätzwerte für die Anzahl der in der Luft enthaltenen Keimlast an den verschiedenen Lagerungsstandorten Seite 30

\section{Tabelle 5:}

Volumeneinstrom durch die wetterabhängigen atmosphärischen Druckänderungen während der entsprechenden Expositionszeit für die vier Kohorten aus den Kornhäusern und die Kontrollgruppe 1

Seite 40

Tabelle 6:

Volumeneinstrom durch die Druckänderungen bei einer Aufzugsfahrt

Seite 40

Tabelle 7:

Betrachtung des resultierenden Gesamtvolumenstroms in das Innere der Verpackung durch die durch Aufzugsfahrten bedingten Druckschwankungen

Seite 41

Tabelle 8:

Resultierendes durch eine Verpackung strömendes Gesamtvolumen während der Expositionszeit (verursacht durch wetterabhängige und durch den Aufzugsbetrieb hervorgerufene Druckänderungen)

Seite 41

Tabelle 9:

Darstellung des durch die Temperaturwechsel verursachten Volumeneinstroms in eine Testeinheit Seite 43

Tabelle 10:

Anteil unsteriler Testeinheiten in den Kornhäusern

Seite 45

Tabelle 11:

Obernjesa ohne Aufzugsbetrieb (,stationäre Lagerung“) - Lufteinstrom pro Verpackung (in ml)

Seite 46

Tabelle 12:

Obernjesa mit Aufzugsbetrieb - Lufteinstrom pro Verpackung (in ml)

Seite 46 
Tabelle 13:

Volumenstrom hervorgerufen durch die atmosphärischen Druckänderungen im Rahmen der Kontrollgruppe 2 (2008)

Seite 51

Tabelle 14:

Volumeneinstrom hervorgerufen durch die atmosphärischen Druckänderungen während der Expositionszeit für eine Testeinheit der drei Kohorten aus Bad Rothenfelde

Seite 51

Tabelle 15:

Volumeneinstrom hervorgerufen durch die atmosphärischen Druckänderungen während der Expositionszeit für eine Testeinheit der drei Kohorten aus Oldenburg

Seite 51

Tabelle 16:

Volumeneinstrom durch Temperaturschwankungen für eine in Bad Rothenfelde gelagerte Testeinheit Seite 53

Tabelle 17:

Volumeneinstrom durch Temperaturschwankungen für eine in Oldenburg gelagerte Testeinheit

Seite 53

Tabelle 18:

Übersicht der gesamten mikrobiologischen Beanspruchung an den verschiedenen Lagerungsstandorten

Seite 56

Tabelle 19:

Vorgaben zur Porosität von Verpackungen und Filtern

Seite 73

Quelle:

Dunkelberg H, Schmelz U (o. J.)

Universitätsmedizin Göttingen

Sterilgut auf höchstem mikrobiologischen Qualitätsniveau verpacken, transportieren und lagern

URL

http://www.sterilgutverpackung-bewerten.de/5e65d9c1a46cab8c47ea8d4cd33679d2_Infomaterial_Haupttext.pdf [Eingesehen am 13.04.2012; 14:23 Uhr] 
6.3.1 Berechnung des Volumenstroms für die in den Kornhäusern gelagerten Testeinheiten und die Kontrollgruppe 1 (2006)

6.3.1.1Volumenstrom durch wetterabhängige Luftdruckänderungen in den Kornhäusern und für die Kontrollgruppe 1 (2006) (Boyle- Mariotte- Gesetz)

Dransfeld (Kornhaus)

Lagerungszeitraum: 07.08.06 - 04.12.06

\begin{tabular}{|c|c|c|c|c|c|c|}
\hline Datum & $\begin{array}{l}\text { Luftdruck } \\
\text { (p1) }\end{array}$ & $\begin{array}{l}\text { Luftdruck des } \\
\text { Folgetages (p2) }\end{array}$ & $\mathrm{V} 2=(\mathrm{p} 1 * \mathrm{~V} 1) / \mathrm{p} 2$ & $\begin{array}{l}\text { Luftvolumen- } \\
\text { strom }\end{array}$ & $\begin{array}{c}\text { Luftvolumenein- } \\
\text { strom } \\
\text { (=postive Werte) }\end{array}$ & $\begin{array}{c}\text { Summe } \\
\Delta \mathrm{V}\end{array}$ \\
\hline & (hPa) & (ml) & (ml) & (ml) & $(\mathbf{m l})$ & (ml) \\
\hline 07.08.2006 & 1011 & 1014 & 239,2899408 & 0,710059172 & 0,710059172 & \\
\hline 08.08.2006 & 1014 & 1012 & 240,4743083 & $-0,4743083$ & & \\
\hline 09.08.2006 & 1012 & 1010 & 240,4752475 & $-0,475247525$ & & \\
\hline 10.08.2006 & 1010 & 1008 & 240,4761905 & $-0,476190476$ & & \\
\hline 11.08 .2006 & 1008 & 1007 & 240,2383317 & $-0,238331678$ & & \\
\hline 12.08.2006 & 1007 & 1005 & 240,4776119 & $-0,47761194$ & & \\
\hline 13.08.2006 & 1005 & 1003 & 240,4785643 & $-0,478564307$ & & \\
\hline 14.08.2006 & 1003 & 1000 & 240,72 & $-0,72$ & & \\
\hline 15.08.2006 & 1000 & 1008 & 238,0952381 & 1,904761905 & 1,904761905 & \\
\hline 16.08.2006 & 1008 & 1005 & 240,7164179 & $-0,71641791$ & & \\
\hline 17.08 .2006 & 1005 & 1002 & 240,7185629 & $-0,718562874$ & & \\
\hline 18.08.2006 & 1002 & 1013 & 237,3938796 & 2,606120434 & 2,606120434 & \\
\hline 19.08.2006 & 1013 & 1015 & 239,5270936 & 0,472906404 & 0,472906404 & \\
\hline 20.08.2006 & 1015 & 1014 & 240,2366864 & $-0,236686391$ & & \\
\hline 21.08.2006 & 1014 & 1012 & 240,4743083 & $-0,4743083$ & & \\
\hline 22.08 .2006 & 1012 & 1016 & 239,0551181 & 0,94488189 & 0,94488189 & \\
\hline 23.08.2006 & 1016 & 1008 & 241,9047619 & $-1,904761905$ & & \\
\hline 24.08.2006 & 1008 & 1005 & 240,7164179 & $-0,71641791$ & & \\
\hline 25.08 .2006 & 1005 & 1007 & 239,5233366 & 0,476663357 & 0,476663357 & \\
\hline 26.08.2006 & 1007 & 1010 & 239,2871287 & 0,712871287 & 0,712871287 & \\
\hline 27.08.2006 & 1010 & 999 & 242,6426426 & $-2,642642643$ & & \\
\hline 28.08.2006 & 999 & 1004 & 238,8047809 & 1,195219124 & 1,195219124 & \\
\hline 29.08.2006 & 1004 & 1013 & 237,8677196 & 2,132280355 & 2,132280355 & \\
\hline 30.08 .2006 & 1013 & 1022 & 237,8864971 & 2,113502935 & 2,113502935 & \\
\hline 31.08 .2006 & 1022 & 1016 & 241,4173228 & $-1,417322835$ & & \\
\hline
\end{tabular}




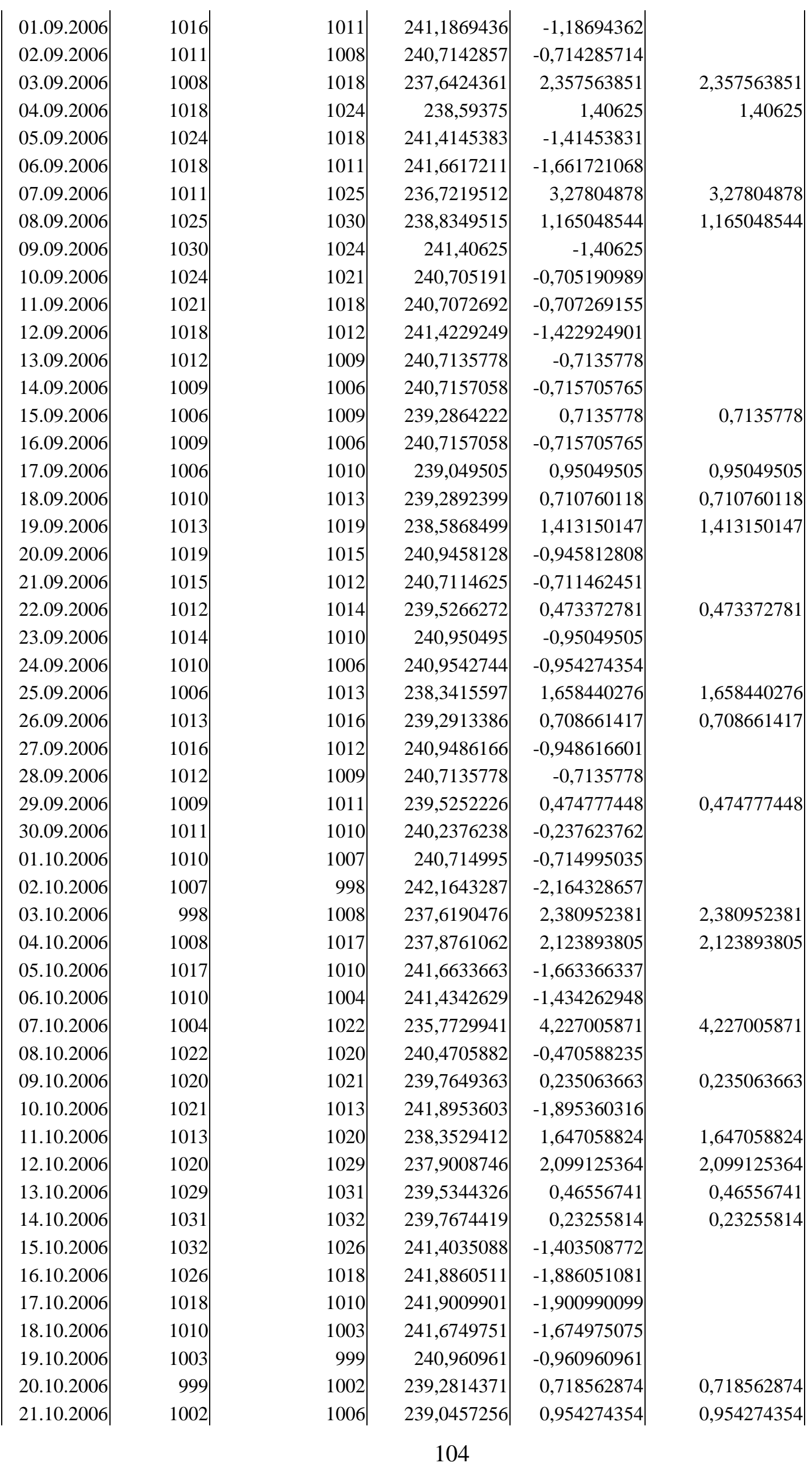




\begin{tabular}{|c|c|c|c|c|c|c|}
\hline 22.10 .2006 & 1006 & 997 & 242,1664995 & $-2,166499498$ & \multirow{4}{*}{5,207100592} & \\
\hline 23.10 .2006 & 997 & 992 & 241,2096774 & $-1,209677419$ & & \\
\hline 24.10 .2006 & 992 & 1014 & 234,7928994 & 5,207100592 & & \\
\hline 25.10 .2006 & 1014 & 1007 & 241,6683217 & $-1,668321748$ & & \\
\hline 26.10 .2006 & 1007 & 1018 & 237,4066798 & 2,593320236 & \multirow{3}{*}{$\begin{array}{l}2,593320236 \\
1,871345029\end{array}$} & \\
\hline 27.10 .2006 & 1018 & 1026 & 238,128655 & 1,871345029 & & \\
\hline 28.10 .2006 & 1026 & 1012 & 243,3201581 & $-3,320158103$ & & \\
\hline 29.10 .2006 & 1012 & 1023 & 237,4193548 & 2,580645161 & \multirow[t]{2}{*}{2,580645161} & \\
\hline 30.10 .2006 & 1023 & 1000 & 245,52 & $-5,52$ & & \\
\hline 31.10 .2006 & 1000 & 1012 & 237,1541502 & 2,845849802 & \multirow{5}{*}{$\begin{array}{l}2,845849802 \\
3,274853801 \\
0,932038835\end{array}$} & \\
\hline 01.11.2006 & 1012 & 1026 & 236,7251462 & 3,274853801 & & \\
\hline 02.11 .2006 & 1026 & 1030 & 239,0679612 & 0,932038835 & & \\
\hline 03.11.2006 & 1030 & 1027 & 240,7010711 & $-0,701071081$ & & \\
\hline 04.11 .2006 & 1027 & 1021 & 241,410382 & $-1,410381978$ & & \\
\hline 05.11 .2006 & 1021 & 1025 & 239,0634146 & 0,936585366 & \multirow[t]{4}{*}{0,936585366} & \\
\hline 06.11.2006 & 1025 & 1023 & 240,4692082 & $-0,469208211$ & & \\
\hline 07.11 .2006 & 1023 & 1019 & 240,9421001 & $-0,942100098$ & & \\
\hline 08.11.2006 & 1019 & 1016 & 240,7086614 & $-0,708661417$ & & \\
\hline 09.11.2006 & 1016 & 1036 & 235,3667954 & 4,633204633 & \multirow[t]{2}{*}{ 4,633204633 } & \\
\hline 10.11.2006 & 1036 & 1006 & 247,1570577 & $-7,157057654$ & & \\
\hline 11.11.2006 & 1006 & 1014 & 238,1065089 & 1,893491124 & \multirow[t]{2}{*}{1,893491124} & \\
\hline 12.11.2006 & 1014 & 1002 & 242,8742515 & $-2,874251497$ & & \\
\hline 13.11.2006 & 1002 & 1009 & 238,3349851 & 1,665014866 & \multirow{3}{*}{$\begin{array}{l}1,665014866 \\
1,183431953\end{array}$} & \\
\hline 14.11.2006 & 1009 & 1014 & 238,816568 & 1,183431953 & & \\
\hline 15.11.2006 & 1014 & 1006 & 241,9085487 & $-1,908548708$ & & \\
\hline 16.11.2006 & 1006 & 1008 & 239,5238095 & 0,476190476 & \multirow{2}{*}{$\begin{array}{l}0,476190476 \\
1,420118343\end{array}$} & \\
\hline 17.11.2006 & 1008 & 1014 & 238,5798817 & 1,420118343 & & \\
\hline 18.11.2006 & 1014 & 1022 & 238,1213307 & 1,878669276 & \multirow[t]{3}{*}{1,878669276} & \\
\hline 19.11.2006 & 1022 & 1012 & 242,3715415 & $-2,371541502$ & & \\
\hline 20.11.2006 & 1012 & 995 & 244,1005025 & $-4,100502513$ & & \\
\hline 21.11.2006 & 995 & 1001 & 238,5614386 & 1,438561439 & \multirow[t]{2}{*}{1,438561439} & \\
\hline 22.11.2006 & 1001 & 993 & 241,9335347 & $-1,933534743$ & & \\
\hline 23.11.2006 & 993 & 1005 & 237,1343284 & 2,865671642 & \multirow[t]{2}{*}{2,865671642} & \\
\hline 24.11.2006 & 1005 & 1002 & 240,7185629 & $-0,718562874$ & & \\
\hline 25.11.2006 & 1002 & 1019 & 235,9960746 & 4,003925417 & \multirow{3}{*}{$\begin{array}{l}4,003925417 \\
0,938416422\end{array}$} & \\
\hline 26.11.2006 & 1019 & 1023 & 239,0615836 & 0,938416422 & & \\
\hline 27.11.2006 & 1023 & 1020 & 240,7058824 & $-0,705882353$ & & \\
\hline 28.11.2006 & 1020 & 1029 & 237,9008746 & 2,099125364 & \multirow{4}{*}{$\begin{array}{l}2,099125364 \\
2,309913378\end{array}$} & \\
\hline 29.11.2006 & 1029 & 1039 & 237,6900866 & 2,309913378 & & \\
\hline 30.11 .2006 & 1039 & 1027 & 242,8042843 & $-2,804284323$ & & \\
\hline 01.12 .2006 & 1027 & 1018 & 242,1218075 & $-2,121807466$ & & \\
\hline 02.12 .2006 & 1018 & 1000 & 244,32 & $-4,32$ & \multirow{3}{*}{ 0,956175299 } & \\
\hline 03.12.2006 & 1000 & 1004 & 239,0438247 & 0,956175299 & & \\
\hline 04.12 .2006 & 1004 & & & & & 91,6671242 \\
\hline
\end{tabular}


Obernjesa (Kornhaus)

Lagerungszeitraum: 07.08.06 - 23.11.06

\begin{tabular}{|c|c|c|c|c|c|c|}
\hline Datum & $\begin{array}{l}\text { Luftdruck } \\
\text { (p1) }\end{array}$ & $\begin{array}{l}\text { Luftdruck des } \\
\text { Folgetages (p2) }\end{array}$ & $V 2=(p 1 * V 1) / p 2$ & \begin{tabular}{|c|}
$\begin{array}{c}\text { Luftvolumen- } \\
\text { strom }\end{array}$ \\
\end{tabular} & $\begin{array}{c}\text { Luftvolumenein- } \\
\text { strom (= positive } \\
\text { Werte) } \\
\end{array}$ & $\begin{array}{c}\text { Summe } \\
\Delta V\end{array}$ \\
\hline & (hPa) & $(\mathrm{ml})$ & $(\mathrm{ml})$ & $(\mathrm{ml})$ & $(\mathbf{m l})$ & $(\mathrm{ml})$ \\
\hline 07.08.2006 & 1011 & 1014 & 239,2899408 & 0,710059172 & 0,710059172 & \\
\hline 08.08.2006 & 1014 & 1012 & 240,4743083 & $-0,4743083$ & & \\
\hline 09.08.2006 & 1012 & 1010 & 240,4752475 & $-0,475247525 \mid$ & & \\
\hline 10.08.2006 & 1010 & 1008 & 240,4761905 & $-0,476190476$ & & \\
\hline 11.08.2006 & 1008 & 1007 & 240,2383317 & $-0,238331678$ & & \\
\hline 12.08.2006 & 1007 & 1005 & 240,4776119 & $-0,47761194$ & & \\
\hline 13.08.2006 & 1005 & 1003 & 240,4785643 & $-0,478564307$ & & \\
\hline 14.08.2006 & 1003 & 1000 & 240,72 & $-0,72$ & & \\
\hline 15.08.2006 & 1000 & 1008 & 238,0952381 & 1,904761905 & 1,904761905 & \\
\hline 16.08.2006 & 1008 & 1005 & 240,7164179 & $-0,71641791$ & & \\
\hline 17.08.2006 & 1005 & 1002 & 240,7185629 & $|-0,718562874|$ & & \\
\hline 18.08.2006 & 1002 & 1013 & 237,3938796 & | 2,606120434 & 2,606120434 & \\
\hline 19.08.2006 & 1013 & 1015 & 239,5270936 & 0,472906404 & 0,472906404 & \\
\hline 20.08.2006 & 1015 & 1014 & 240,2366864 & $-0,236686391$ & & \\
\hline 21.08.2006 & 1014 & 1012 & 240,4743083 & $-0,4743083$ & & \\
\hline 22.08.2006 & 1012 & 1016 & 239,0551181 & 0,94488189 & 0,94488189 & \\
\hline 23.08.2006 & 1016 & 1008 & 241,9047619 & $-1,904761905$ & & \\
\hline 24.08.2006 & 1008 & 1005 & 240,7164179 & $-0,71641791$ & & \\
\hline 25.08.2006 & 1005 & 1007 & 239,5233366 & 0,476663357 & 0,476663357 & \\
\hline 26.08.2006 & 1007 & 1010 & 239,2871287 & 0,712871287 & 0,712871287 & \\
\hline 27.08.2006 & 1010 & 999 & 242,6426426 & $-2,642642643$ & & \\
\hline 28.08.2006 & 999 & 1004 & 238,8047809 & 1,195219124 & 1,195219124 & \\
\hline 29.08.2006 & 1004 & 1013 & 237,8677196 & 2,132280355 & 2,132280355 & \\
\hline 30.08 .2006 & 1013 & 1022 & 237,8864971 & 2,113502935 & 2,113502935 & \\
\hline 31.08.2006 & 1022 & 1016 & 241,4173228 & $-1,417322835$ & & \\
\hline 01.09 .2006 & 1016 & 1011 & 241,1869436 & $-1,18694362$ & & \\
\hline 02.09.2006 & 1011 & 1008 & 240,7142857 & $\mid-0,714285714$ & & \\
\hline 03.09 .2006 & 1008 & 1018 & 237,6424361 & 2,357563851 & 2,357563851 & \\
\hline 04.09 .2006 & 1018 & 1024 & 238,59375 & 1,40625 & 1,40625 & \\
\hline 05.09 .2006 & 1024 & 1018 & 241,4145383 & $-1,41453831$ & & \\
\hline 06.09.2006 & 1018 & 1011 & 241,6617211 & $-1,661721068$ & & \\
\hline 07.09 .2006 & 1011 & 1025 & 236,7219512 & 3,27804878 & 3,27804878 & \\
\hline 08.09.2006 & 1025 & 1030 & 238,8349515 & 1,165048544 & 1,165048544 & \\
\hline 09.09.2006 & 1030 & 1024 & 241,40625 & $-1,40625$ & & \\
\hline 10.09.2006 & 1024 & 1021 & 240,705191 & $-0,705190989$ & & \\
\hline 11.09.2006 & 1021 & 1018 & 240,7072692 & $-0,707269155$ & & \\
\hline 12.09 .2006 & 1018 & 1012 & 241,4229249 & $-1,422924901$ & & \\
\hline 13.09.2006 & 1012 & 1009 & 240,7135778 & $-0,7135778$ & & \\
\hline 14.09 .2006 & 1009 & 1006 & 240,7157058 & $-0,715705765$ & & \\
\hline 15.09.2006 & 1006 & 1009 & 239,2864222 & 0,7135778 & 0,7135778 & \\
\hline 16.09 .2006 & 1009 & 1006 & 240,7157058 & $-0,715705765$ & & \\
\hline 17.09.2006 & 1006 & 1010 & 239,049505 & 0,95049505 & 0,95049505 & \\
\hline 18.09.2006 & 1010 & 1013 & 239,2892399 & 0,710760118 & 0,710760118 & \\
\hline 19.09.2006 & 1013 & 1019 & 238,5868499 & 1,413150147 & 1,413150147 & \\
\hline 20.09.2006 & 1019 & 1015 & 240,9458128 & $-0,945812808$ & & \\
\hline 21.09.2006 & 1015 & 1012 & 240,7114625 & $-0,711462451$ & & \\
\hline 22.09.2006 & 1012 & 1014 & 239,5266272 & 0,473372781 & 0,473372781 & \\
\hline
\end{tabular}




\begin{tabular}{|c|c|c|c|c|c|}
\hline 23.09 .2006 & 1014 & 1010 & 240,950495 & $-0,95049505$ & \multirow{4}{*}{$\begin{array}{l}1,658440276 \\
0,708661417\end{array}$} \\
\hline 24.09 .2006 & 1010 & 1006 & 240,9542744 & $-0,954274354$ & \\
\hline 25.09 .2006 & 1006 & 1013 & 238,3415597 & 1,658440276 & \\
\hline 26.09 .2006 & 1013 & 1016 & 239,2913386 & 0,708661417 & \\
\hline 27.09 .2006 & 1016 & 1012 & 240,9486166 & $-0,948616601$ & \multirow{4}{*}{0,474777448} \\
\hline 28.09 .2006 & 1012 & 1009 & 240,7135778 & $-0,7135778$ & \\
\hline 29.09 .2006 & 1009 & 1011 & 239,5252226 & 0,474777448 & \\
\hline 30.09 .2006 & 1011 & 1010 & 240,2376238 & $-0,237623762$ & \\
\hline 01.10 .2006 & 1010 & 1007 & 240,714995 & $-0,714995035$ & \multirow{5}{*}{$\begin{array}{l}2,380952381 \\
2,123893805\end{array}$} \\
\hline 02.10 .2006 & 1007 & 998 & 242,1643287 & $-2,164328657$ & \\
\hline 03.10 .2006 & 998 & 1008 & 237,6190476 & 2,380952381 & \\
\hline 04.10 .2006 & 1008 & 1017 & 237,8761062 & 2,123893805 & \\
\hline 05.10 .2006 & 1017 & 1010 & 241,6633663 & $-1,663366337$ & \\
\hline 06.10 .2006 & 1010 & 1004 & 241,4342629 & $-1,434262948$ & \multirow{3}{*}{4,227005871} \\
\hline 07.10 .2006 & 1004 & 1022 & 235,7729941 & 4,227005871 & \\
\hline 08.10 .2006 & 1022 & 1020 & 240,4705882 & $-0,470588235$ & \\
\hline 09.10 .2006 & 1020 & 1021 & 239,7649363 & 0,235063663 & \multirow[t]{2}{*}{0,235063663} \\
\hline 10.10 .2006 & 1021 & 1013 & 241,8953603 & $-1,895360316$ & \\
\hline 11.10 .2006 & 1013 & 1020 & 238,3529412 & 1,647058824 & \multirow{5}{*}{$\begin{array}{r}1,647058824 \\
2,099125364 \\
0,46556741 \\
0,23255814\end{array}$} \\
\hline 12.10 .2006 & 1020 & 1029 & 237,9008746 & 2,099125364 & \\
\hline 13.10 .2006 & 1029 & 1031 & 239,5344326 & 0,46556741 & \\
\hline 14.10.2006 & 1031 & 1032 & 239,7674419 & 0,23255814 & \\
\hline 15.10 .2006 & 1032 & 1026 & 241,4035088 & $-1,403508772$ & \\
\hline 16.10.2006 & 1026 & 1018 & 241,8860511 & $-1,886051081$ & \multirow{7}{*}{$\begin{array}{l}0,718562874 \\
0,954274354\end{array}$} \\
\hline 17.10.2006 & 1018 & 1010 & 241,9009901 & $-1,900990099$ & \\
\hline 18.10.2006 & 1010 & 1003 & 241,6749751 & $-1,674975075$ & \\
\hline 19.10.2006 & 1003 & 999 & 240,960961 & $-0,960960961$ & \\
\hline 20.10 .2006 & 999 & 1002 & 239,2814371 & 0,718562874 & \\
\hline 21.10 .2006 & 1002 & 1006 & 239,0457256 & 0,954274354 & \\
\hline 22.10 .2006 & 1006 & 997 & 242,1664995 & $-2,166499498$ & \\
\hline 23.10.2006 & 997 & 992 & 241,2096774 & $-1,209677419$ & \multirow{3}{*}{5,207100592} \\
\hline 24.10 .2006 & 992 & 1014 & 234,7928994 & 5,207100592 & \\
\hline 25.10 .2006 & 1014 & 1007 & 241,6683217 & $-1,668321748$ & \\
\hline 26.10 .2006 & 1007 & 1018 & 237,4066798 & 2,593320236 & \multirow{3}{*}{$\begin{array}{l}2,593320236 \\
1,871345029\end{array}$} \\
\hline 27.10 .2006 & 1018 & 1026 & 238,128655 & 1,871345029 & \\
\hline 28.10 .2006 & 1026 & 1012 & 243,3201581 & $-3,320158103$ & \\
\hline 29.10 .2006 & 1012 & 1023 & 237,4193548 & 2,580645161 & \multirow[t]{2}{*}{2,580645161} \\
\hline 30.10 .2006 & 1023 & 1000 & 245,52 & $-5,52$ & \\
\hline 31.10 .2006 & 1000 & 1012 & 237,1541502 & 2,845849802 & 2,845849802 \\
\hline 01.11 .2006 & 1012 & 1026 & 236,7251462 & 3,274853801 & 3,274853801 \\
\hline 02.11 .2006 & 1026 & 1030 & 239,0679612 & 0,932038835 & \multirow[t]{2}{*}{0,932038835} \\
\hline 03.11 .2006 & 1030 & 1027 & 240,7010711 & $-0,701071081$ & \\
\hline 04.11 .2006 & 1027 & 1021 & 241,410382 & $-1,410381978$ & \multirow{3}{*}{0,936585366} \\
\hline 05.11 .2006 & 1021 & 1025 & 239,0634146 & 0,936585366 & \\
\hline 06.11 .2006 & 1025 & 1023 & 240,4692082 & $-0,469208211$ & \\
\hline 07.11 .2006 & 1023 & 1019 & 240,9421001 & $-0,942100098$ & \multirow{4}{*}{ 4,633204633 } \\
\hline 08.11 .2006 & 1019 & 1016 & 240,7086614 & $-0,708661417$ & \\
\hline 09.11 .2006 & 1016 & 1036 & 235,3667954 & 4,633204633 & \\
\hline 10.11.2006 & 1036 & 1006 & 247,1570577 & $-7,157057654$ & \\
\hline 11.11 .2006 & 1006 & 1014 & 238,1065089 & 1,893491124 & \multirow[t]{2}{*}{1,893491124} \\
\hline 12.11 .2006 & 1014 & 1002 & 242,8742515 & $-2,874251497$ & \\
\hline 13.11.2006 & 1002 & 1009 & 238,3349851 & 1,665014866 & 1,665014866 \\
\hline 14.11.2006 & 1009 & 1014 & 238,816568 & 1,183431953 & \multirow{2}{*}{1,183431953} \\
\hline 15.11 .2006 & 1014 & 1006 & 241,9085487 & $-1,908548708$ & \\
\hline 16.11.2006 & 1006 & 1008 & 239,5238095 & 0,476190476 & 0,476190476 \\
\hline 17.11.2006 & 1008 & 1014 & 238,5798817 & 1,420118343 & 1,420118343 \\
\hline 18.11.2006 & 1014 & 1022 & 238,1213307 & 1,878669276 & 1,878669276 \\
\hline & & & 107 & & \\
\hline
\end{tabular}




\begin{tabular}{|r|r|r|r|r|r|r|}
19.11 .2006 & 1022 & 1012 & 242,3715415 & $-2,371541502$ & & \\
20.11 .2006 & 1012 & 995 & 244,1005025 & $-4,100502513$ & \\
21.11 .2006 & 995 & 1001 & 238,5614386 & 1,438561439 & 1,438561439 & \\
22.11 .2006 & 1001 & 993 & 241,9335347 & $-1,933534743$ & & \\
23.11 .2006 & 993 & & & & & 78,493896 \\
& & & & & 7 \\
\hline
\end{tabular}

Scharzfeld (Kornhaus)

Lagerungszeitraum: 07.08.06 - 23.11.06

\begin{tabular}{|c|c|c|c|c|c|c|}
\hline Datum & $\begin{array}{l}\text { Luftdruck } \\
\text { (p1) }\end{array}$ & \begin{tabular}{|c|}
$\begin{array}{c}\text { Luftdruck } \\
\text { des Folgetages } \\
\text { (p2) }\end{array}$ \\
\end{tabular} & $\mathrm{V} 2=(\mathrm{p} 1 * \mathrm{~V} 1) / \mathrm{p} 2$ & $\begin{array}{c}\text { Luftvolumen- } \\
\text { strom }\end{array}$ & $\begin{array}{c}\text { Luftvolumenein- } \\
\text { strom } \\
\text { (= positive Werte) }\end{array}$ & $\begin{array}{l}\text { Summe } \\
\quad \Delta V\end{array}$ \\
\hline & (hPa) & (ml) & (ml) & (ml) & \begin{tabular}{|l|} 
\\
$(\mathrm{ml})$ \\
\end{tabular} & (ml) \\
\hline 07.08.2006 & 1011 & 1014 & 239,2899408 & 0,71005917 & \multirow[t]{8}{*}{0,710059} & \\
\hline 08.08.2006 & 1014 & 1012 & 240,4743083 & $-0,4743083$ & & \\
\hline 09.08.2006 & 1012 & 1010 & 240,4752475 & $-0,47524752$ & & \\
\hline 10.08 .2006 & 1010 & 1008 & 240,4761905 & $-0,47619048$ & & \\
\hline 11.08.2006 & 1008 & 1007 & 240,2383317 & $-0,23833168$ & & \\
\hline 12.08 .2006 & 1007 & 1005 & 240,4776119 & $-0,47761194$ & & \\
\hline 13.08.2006 & 1005 & 1003 & 240,4785643 & $-0,47856431$ & & \\
\hline 14.08.2006 & 1003 & 1000 & 240,72 & $-0,72$ & & \\
\hline 15.08.2006 & 1000 & 1008 & 238,0952381 & 1,9047619 & \multirow[t]{3}{*}{ 1,904762 } & \\
\hline 16.08.2006 & 1008 & 1005 & 240,7164179 & $-0,71641791$ & & \\
\hline 17.08.2006 & 1005 & 1002 & 240,7185629 & $-0,71856287$ & & \\
\hline 18.08.2006 & 1002 & 1013 & 237,3938796 & 2,60612043 & \multirow{4}{*}{$\begin{array}{r}2,60612 \\
0,472906\end{array}$} & \\
\hline 19.08.2006 & 1013 & 1015 & 239,5270936 & 0,4729064 & & \\
\hline 20.08.2006 & 1015 & 1014 & 240,2366864 & $-0,23668639$ & & \\
\hline 21.08.2006 & 1014 & 1012 & 240,4743083 & $-0,4743083$ & & \\
\hline 22.08.2006 & 1012 & 1016 & 239,0551181 & 0,94488189 & \multirow[t]{3}{*}{0,944882} & \\
\hline 23.08.2006 & 1016 & 1008 & 241,9047619 & $-1,9047619$ & & \\
\hline 24.08.2006 & 1008 & 1005 & 240,7164179 & $-0,71641791$ & & \\
\hline 25.08.2006 & 1005 & 1007 & 239,5233366 & 0,47666336 & \multirow{3}{*}{$\begin{array}{l}0,476663 \\
0,712871\end{array}$} & \\
\hline 26.08 .2006 & 1007 & 1010 & 239,2871287 & 0,71287129 & & \\
\hline 27.08.2006 & 1010 & 999 & 242,6426426 & $-2,64264264$ & & \\
\hline 28.08.2006 & 999 & 1004 & 238,8047809 & 1,19521912 & \multirow{6}{*}{$\begin{array}{r}1,195219 \\
2,13228 \\
2,113503\end{array}$} & \\
\hline 29.08.2006 & 1004 & 1013 & 237,8677196 & 2,13228036 & & \\
\hline 30.08 .2006 & 1013 & 1022 & 237,8864971 & 2,11350294 & & \\
\hline 31.08.2006 & 1022 & 1016 & 241,4173228 & $-1,41732283$ & & \\
\hline 01.09.2006 & 1016 & 1011 & 241,1869436 & $-1,18694362$ & & \\
\hline 02.09.2006 & 1011 & 1008 & 240,7142857 & $-0,71428571$ & & \\
\hline 03.09.2006 & 1008 & 1018 & 237,6424361 & 2,35756385 & \multirow{4}{*}{$\begin{array}{r}2,357564 \\
1,40625\end{array}$} & \\
\hline 04.09 .2006 & 1018 & 1024 & 238,59375 & 1,40625 & & \\
\hline 05.09 .2006 & 1024 & 1018 & 241,4145383 & $-1,41453831$ & & \\
\hline 06.09.2006 & 1018 & 1011 & 241,6617211 & $-1,66172107$ & & \\
\hline
\end{tabular}




\begin{tabular}{|c|c|c|c|c|c|}
\hline 07.09.2006 & 1011 & 1025 & 236,7219512 & 3,27804878 & 3,278049 \\
\hline 08.09.2006 & 1025 & 1030 & 238,8349515 & 1,16504854 & 1,165049 \\
\hline 09.09.2006 & 1030 & 1024 & 241,40625 & $-1,40625$ & \\
\hline 10.09 .2006 & 1024 & 1021 & 240,705191 & $-0,70519099$ & \\
\hline 11.09.2006 & 1021 & 1018 & 240,7072692 & $-0,70726916$ & \\
\hline 12.09 .2006 & 1018 & 1012 & 241,4229249 & $-1,4229249$ & \\
\hline 13.09.2006 & 1012 & 1009 & 240,7135778 & $-0,7135778$ & \\
\hline 14.09 .2006 & 1009 & 1006 & 240,7157058 & $-0,71570577$ & \\
\hline 15.09 .2006 & 1006 & 1009 & 239,2864222 & 0,7135778 & 0,713578 \\
\hline 16.09 .2006 & 1009 & 1006 & 240,7157058 & $-0,71570577$ & \\
\hline 17.09 .2006 & 1006 & 1010 & 239,049505 & 0,95049505 & 0,950495 \\
\hline 18.09.2006 & 1010 & 1013 & 239,2892399 & 0,71076012 & 0,71076 \\
\hline 19.09 .2006 & 1013 & 1019 & 238,5868499 & 1,41315015 & 1,41315 \\
\hline 20.09.2006 & 1019 & 1015 & 240,9458128 & $-0,94581281$ & \\
\hline 21.09.2006 & 1015 & 1012 & 240,7114625 & $-0,71146245$ & \\
\hline 22.09 .2006 & 1012 & 1014 & 239,5266272 & 0,47337278 & 0,473373 \\
\hline 23.09.2006 & 1014 & 1010 & 240,950495 & $-0,95049505$ & \\
\hline 24.09.2006 & 1010 & 1006 & 240,9542744 & $-0,95427435$ & \\
\hline 25.09.2006 & 1006 & 1013 & 238,3415597 & 1,65844028 & 1,65844 \\
\hline 26.09.2006 & 1013 & 1016 & 239,2913386 & 0,70866142 & 0,708661 \\
\hline 27.09.2006 & 1016 & 1012 & 240,9486166 & $-0,9486166$ & \\
\hline 28.09.2006 & 1012 & 1009 & 240,7135778 & $-0,7135778$ & \\
\hline 29.09 .2006 & 1009 & 1011 & 239,5252226 & 0,47477745 & 0,474777 \\
\hline 30.09 .2006 & 1011 & 1010 & 240,2376238 & $-0,23762376$ & \\
\hline 01.10 .2006 & 1010 & 1007 & 240,714995 & $-0,71499503$ & \\
\hline 02.10 .2006 & 1007 & 998 & 242,1643287 & $-2,16432866$ & \\
\hline 03.10 .2006 & 998 & 1008 & 237,6190476 & 2,38095238 & 2,380952 \\
\hline 04.10 .2006 & 1008 & 1017 & 237,8761062 & 2,12389381 & 2,123894 \\
\hline 05.10 .2006 & 1017 & 1010 & 241,6633663 & $-1,66336634$ & \\
\hline 06.10 .2006 & 1010 & 1004 & 241,4342629 & $-1,43426295$ & \\
\hline 07.10 .2006 & 1004 & 1022 & 235,7729941 & 4,22700587 & 4,227006 \\
\hline 08.10 .2006 & 1022 & 1020 & 240,4705882 & $-0,47058824$ & \\
\hline 09.10 .2006 & 1020 & 1021 & 239,7649363 & 0,23506366 & 0,235064 \\
\hline 10.10 .2006 & 1021 & 1013 & 241,8953603 & $-1,89536032$ & \\
\hline 11.10 .2006 & 1013 & 1020 & 238,3529412 & 1,64705882 & 1,647059 \\
\hline 12.10 .2006 & 1020 & 1029 & 237,9008746 & 2,09912536 & 2,099125 \\
\hline 13.10.2006 & 1029 & 1031 & 239,5344326 & 0,46556741 & 0,465567 \\
\hline 14.10 .2006 & 1031 & 1032 & 239,7674419 & 0,23255814 & 0,232558 \\
\hline 15.10 .2006 & 1032 & 1026 & 241,4035088 & $-1,40350877$ & \\
\hline 16.10 .2006 & 1026 & 1018 & 241,8860511 & $-1,88605108$ & \\
\hline 17.10.2006 & 1018 & 1010 & 241,9009901 & $-1,9009901$ & \\
\hline 18.10.2006 & 1010 & 1003 & 241,6749751 & $-1,67497507$ & \\
\hline 19.10.2006 & 1003 & 999 & 240,960961 & $-0,96096096$ & \\
\hline 20.10 .2006 & 999 & 1002 & 239,2814371 & 0,71856287 & 0,718563 \\
\hline 21.10 .2006 & 1002 & 1006 & 239,0457256 & 0,95427435 & 0,954274 \\
\hline 22.10 .2006 & 1006 & 997 & 242,1664995 & $-2,1664995$ & \\
\hline 23.10 .2006 & 997 & 992 & 241,2096774 & $-1,20967742$ & \\
\hline 24.10 .2006 & 992 & 1014 & 234,7928994 & 5,20710059 & 5,207101 \\
\hline 25.10 .2006 & 1014 & 1007 & 241,6683217 & $-1,66832175$ & \\
\hline 26.10 .2006 & 1007 & 1018 & 237,4066798 & 2,59332024 & 2,59332 \\
\hline 27.10 .2006 & 1018 & 1026 & 238,128655 & 1,87134503 & 1,871345 \\
\hline
\end{tabular}




\begin{tabular}{|c|c|c|c|c|c|c|}
\hline 28.10 .2006 & 1026 & 1012 & 243,3201581 & $-3,3201581$ & \multirow[b]{2}{*}{2,580645} & \\
\hline 29.10 .2006 & 1012 & 1023 & 237,4193548 & 2,58064516 & & \\
\hline 30.10 .2006 & 1023 & 1000 & 245,52 & $-5,52$ & & \\
\hline 31.10 .2006 & 1000 & 1012 & 237,1541502 & 2,8458498 & 2,84585 & \\
\hline 01.11.2006 & 1012 & 1026 & 236,7251462 & 3,2748538 & 3,274854 & \\
\hline 02.11 .2006 & 1026 & 1030 & 239,0679612 & 0,93203883 & 0,932039 & \\
\hline 03.11.2006 & 1030 & 1027 & 240,7010711 & $-0,70107108$ & \multirow{6}{*}{0,936585} & \\
\hline 04.11 .2006 & 1027 & 1021 & 241,410382 & $-1,41038198$ & & \\
\hline 05.11.2006 & 1021 & 1025 & 239,0634146 & 0,93658537 & & \\
\hline 06.11 .2006 & 1025 & 1023 & 240,4692082 & $-0,46920821$ & & \\
\hline 07.11 .2006 & 1023 & 1019 & 240,9421001 & $-0,9421001$ & & \\
\hline 08.11 .2006 & 1019 & 1016 & 240,7086614 & $-0,70866142$ & & \\
\hline 09.11.2006 & 1016 & 1036 & 235,3667954 & 4,63320463 & \multirow[t]{2}{*}{ 4,633205 } & \\
\hline 10.11 .2006 & 1036 & 1006 & 247,1570577 & $-7,15705765$ & & \\
\hline 11.11.2006 & 1006 & 1014 & 238,1065089 & 1,89349112 & \multirow[t]{2}{*}{1,893491} & \\
\hline 12.11.2006 & 1014 & 1002 & 242,8742515 & $-2,8742515$ & & \\
\hline 13.11.2006 & 1002 & 1009 & 238,3349851 & 1,66501487 & \multirow{3}{*}{$\begin{array}{l}1,665015 \\
1,183432\end{array}$} & \\
\hline 14.11.2006 & 1009 & 1014 & 238,816568 & 1,18343195 & & \\
\hline 15.11.2006 & 1014 & 1006 & 241,9085487 & $-1,90854871$ & & \\
\hline 16.11.2006 & 1006 & 1008 & 239,5238095 & 0,47619048 & \multirow{5}{*}{$\begin{array}{r}0,47619 \\
1,420118 \\
1,878669\end{array}$} & \\
\hline 17.11.2006 & 1008 & 1014 & 238,5798817 & 1,42011834 & & \\
\hline 18.11.2006 & 1014 & 1022 & 238,1213307 & 1,87866928 & & \\
\hline 19.11.2006 & 1022 & 1012 & 242,3715415 & $-2,3715415$ & & \\
\hline 20.11.2006 & 1012 & 995 & 244,1005025 & $-4,10050251$ & & \\
\hline 21.11.2006 & 995 & 1001 & 238,5614386 & 1,43856144 & \multirow[t]{3}{*}{1,438561} & \\
\hline 22.11 .2006 & 1001 & 993 & 241,9335347 & $-1,93353474$ & & \\
\hline 23.11.2006 & 993 & & & & & 78,4938967 \\
\hline
\end{tabular}

Göttingen (Kontrollgruppe 1 (2006))

Lagerungszeitraum: 10.06.06 - 17.11.06

\begin{tabular}{|c|c|c|c|c|c|c|}
\hline Datum & $\begin{array}{c}\text { Luftdruck } \\
\text { (p1) }\end{array}$ & \begin{tabular}{|c|}
$\begin{array}{c}\text { Luftdruck } \\
\text { des Folgetages } \\
\text { (p2) }\end{array}$ \\
\end{tabular} & $V 2=(p 1 * V 1) / p 2$ & $\begin{array}{l}\text { Luftvolumen- } \\
\text { strom }\end{array}$ & $\begin{array}{c}\begin{array}{c}\text { Lufteinstrom- } \\
\text { volumen } \\
\text { (= positiver Werte) }\end{array} \\
\end{array}$ & $\begin{array}{c}\text { Summe } \\
\Delta \mathrm{V}\end{array}$ \\
\hline & (hPa) & $(\mathbf{m l})$ & (ml) & $(\mathrm{ml})$ & (ml) & (ml) \\
\hline 10.06.2006 & 1023 & 1028 & 238,8326848 & 1,167315175 & 1,16732 & \\
\hline 11.06.2006 & 1028 & 1025 & 240,702439 & $-0,702439024$ & & \\
\hline 12.06.2006 & 1025 & 1020 & 241,1764706 & $-1,176470588$ & & \\
\hline 13.06.2006 & 1020 & 1014 & 241,4201183 & $-1,420118343$ & & \\
\hline 14.06.2006 & 1014 & 1015 & 239,7635468 & 0,236453202 & 0,23645 & \\
\hline 15.06.2006 & 1015 & 1016 & 239,7637795 & 0,236220472 & 0,23622 & \\
\hline 16.06.2006 & 1016 & 1019 & 239,2934249 & 0,706575074 & 0,70658 & \\
\hline 17.06.2006 & 1019 & 1015 & 240,9458128 & $-0,945812808$ & & \\
\hline 18.06.2006 & 1015 & 1010 & 241,1881188 & $-1,188118812$ & & \\
\hline 19.06.2006 & 1010 & 1015 & 238,817734 & 1,18226601 & 1,18227 & \\
\hline
\end{tabular}




\begin{tabular}{|c|c|c|c|}
\hline 20.06.2006 & 1015 & 1008 & 241,666666 \\
\hline 21.06.2006 & 1008 & 1011 & 239,287833 \\
\hline 22.06.2006 & 1011 & 1019 & 238,11579 \\
\hline 23.06.2006 & 1019 & 1016 & 240,70866 \\
\hline 24.06 .2006 & 1016 & 1011 & 241,18694 \\
\hline 25.06.2006 & 1011 & 1008 & 240,71428 \\
\hline 26.06.2006 & 1008 & 1017 & 237,87610 \\
\hline 27.06.2006 & 1017 & 1021 & 239,05974 \\
\hline 28.06.2006 & 1021 & 1022 & 239,76516 \\
\hline 29.06.2006 & 1022 & 1024 & 239,531 \\
\hline 30.06 .2006 & 1024 & 1026 & 239,53216 \\
\hline 01.07 .2006 & 1026 & 1022 & 240,93933 \\
\hline 02.07 .2006 & 1022 & 1018 & 240,94302 \\
\hline 03.07.2006 & 1018 & 1015 & 240,70935 \\
\hline 04.07 .2006 & 1015 & 1012 & 240,71146 \\
\hline 05.07.2006 & 1012 & 1015 & 239,29064 \\
\hline 06.07.2006 & 1015 & 1015 & \\
\hline 07.07 .2006 & 1015 & 1017 & 239,52802 \\
\hline 08.07.2006 & 1017 & 1016 & 240,23622 \\
\hline 09.07.2006 & 1016 & 1023 & 238,35777 \\
\hline 10.07 .2006 & 1023 & 1019 & 240,94210 \\
\hline 11.07.2006 & 1019 & 1025 & 238,5951 \\
\hline 12.07 .2006 & 1025 & 1018 & 241,65029 \\
\hline 13.07.2006 & 1018 & 1026 & 238,1286 \\
\hline 14.07.2006 & 1026 & 1030 & 239,06796 \\
\hline 15.07.2006 & 1030 & 1029 & 240,23323 \\
\hline 16.07.2006 & 1029 & 1025 & 240,93658 \\
\hline 17.07.2006 & 1025 & 1021 & 240,94025 \\
\hline 18.07.2006 & 1021 & 1018 & 240,70726 \\
\hline 19.07.2006 & 1018 & 1014 & 240,94674 \\
\hline 20.07.2006 & 1014 & 1019 & 238,82237 \\
\hline 21.07.2006 & 1019 & 1013 & 241,42152 \\
\hline 22.07.2006 & 1013 & 1017 & 239,05604 \\
\hline 23.07.2006 & 1017 & 1018 & 239,76424 \\
\hline 24.07.2006 & 1018 & 1019 & 239,7644 \\
\hline 25.07.2006 & 1019 & 1015 & 240,94581 \\
\hline 26.07.2006 & 1015 & 1012 & 240,71146 \\
\hline 27.07.2006 & 1012 & 1008 & 240,9523 \\
\hline 28.07.2006 & 1008 & 1007 & 240,23833 \\
\hline 29.07 .2006 & 1007 & 1011 & 239,05044 \\
\hline 30.07 .2006 & 1011 & 1013 & 239,52615 \\
\hline 31.07 .2006 & 1013 & 1003 & 242,39282 \\
\hline 01.08.2006 & 1003 & 1005 & 239,52238 \\
\hline 02.08.2006 & 1005 & 1003 & 240,47856 \\
\hline 03.08.2006 & 1003 & 1008 & 238,80952 \\
\hline 04.08 .2006 & 1008 & 1013 & 238,81539 \\
\hline 05.08.2006 & 1013 & 1013 & \\
\hline 06.08.2006 & 1013 & 1011 & 240,47477 \\
\hline 07.08 .2006 & 1011 & 1014 & 239,28994 \\
\hline 08.08.2006 & 1014 & 1012 & 240,4743 \\
\hline 09.08.2006 & 1012 & 1010 & 240,47524 \\
\hline
\end{tabular}

\begin{tabular}{|c|c|}
\hline \multicolumn{2}{|l|}{$-1,6666666667$} \\
\hline 0,712166172 & 0,71217 \\
\hline 1,884200196 & 1,8842 \\
\hline \multicolumn{2}{|l|}{$-0,708661417$} \\
\hline \multicolumn{2}{|l|}{$-1,18694362$} \\
\hline \multicolumn{2}{|l|}{$-0,714285714$} \\
\hline 2,123893805 & 2,12389 \\
\hline 0,940254652 & 0,94025 \\
\hline 0,234833659 & 0,23483 \\
\hline 0,46875 & 0,46875 \\
\hline 0,467836257 & 0,46784 \\
\hline \multicolumn{2}{|l|}{$-0,939334638$} \\
\hline \multicolumn{2}{|l|}{$-0,94302554$} \\
\hline \multicolumn{2}{|l|}{$-0,709359606$} \\
\hline \multicolumn{2}{|l|}{$-0,711462451$} \\
\hline \multirow{2}{*}{\multicolumn{2}{|c|}{$\begin{array}{r}0,709359606 \\
0\end{array}$}} \\
\hline & \\
\hline \multicolumn{2}{|l|}{0,471976401} \\
\hline \multicolumn{2}{|l|}{$-0,236220472$} \\
\hline 1,642228739 & 1,64223 \\
\hline \multicolumn{2}{|l|}{$-0,942100098$} \\
\hline 1,404878049 & 1,40488 \\
\hline \multicolumn{2}{|l|}{$-1,650294695$} \\
\hline 1,871345029 & 1,87135 \\
\hline 0,932038835 & 0,93204 \\
\hline \multicolumn{2}{|l|}{$-0,233236152$} \\
\hline \multicolumn{2}{|l|}{$-0,936585366$} \\
\hline \multicolumn{2}{|l|}{$-0,940254652$} \\
\hline \multicolumn{2}{|l|}{$-0,707269155$} \\
\hline \multicolumn{2}{|l|}{$-0,9467455562$} \\
\hline 1,177625123 & 1,17763 \\
\hline \multicolumn{2}{|l|}{$-1,421520237$} \\
\hline 0,943952802 & 0,94395 \\
\hline 0,235756385 & 0,23576 \\
\hline 0,235525025 & 0,23553 \\
\hline \multicolumn{2}{|l|}{$-0,945812808$} \\
\hline \multicolumn{2}{|l|}{$-0,711462451$} \\
\hline \multicolumn{2}{|l|}{$-0,952380952$} \\
\hline \multicolumn{2}{|l|}{$-0,238331678$} \\
\hline 0,949554896 & 0,94955 \\
\hline 0,473840079 & 0,47384 \\
\hline$-2,392821535$ & \\
\hline 0,47761194 & 0,47761 \\
\hline$-0,478564307$ & \\
\hline 1,19047619 & 1,19048 \\
\hline 1,184600197 & 1,1846 \\
\hline 0 & \\
\hline$-0,474777448$ & \\
\hline 0,710059172 & 0,71006 \\
\hline$-0,4743083$ & \\
\hline$-0,475247525$ & \\
\hline
\end{tabular}




\begin{tabular}{|c|c|c|c|}
\hline 10.08.2006 & 1010 & 1008 & 240,4761905 \\
\hline 11.08.2006 & 1008 & 1007 & 240,2383317 \\
\hline 12.08.2006 & 1007 & 1005 & 240,4776119 \\
\hline 13.08.2006 & 1005 & 1003 & 240,47856 \\
\hline 14.08.2006 & 1003 & 1000 & 240 \\
\hline 15.08.2006 & 1000 & 1008 & 238,09523 \\
\hline 16.08 .2006 & 1008 & 1005 & 240,71641 \\
\hline 17.08.2006 & 1005 & 1002 & 240,7185629 \\
\hline 18.08.2006 & 1002 & 1013 & 237,39387 \\
\hline 19.08.2006 & 1013 & 1015 & 239,52709 \\
\hline 20.08.2006 & 1015 & 1014 & 240,23668 \\
\hline 21.08.2006 & 1014 & 1012 & 240,474308 \\
\hline 22.08 .2006 & 1012 & 1016 & 239,055118 \\
\hline 23.08.2006 & 1016 & 1008 & 241,9047619 \\
\hline 24.08 .2006 & 1008 & 1005 & 240,716417 \\
\hline 25.08.2006 & 1005 & 1007 & 239,5233366 \\
\hline 26.08.2006 & 1007 & 1010 & 239,287128 \\
\hline 27.08.2006 & 1010 & 999 & 242,64264 \\
\hline 28.08.2006 & 999 & 1004 & 238,804780 \\
\hline 29.08.2006 & 1004 & 1013 & 237,8677196 \\
\hline 30.08 .2006 & 1013 & 1022 & 237,88649 \\
\hline 31.08.2006 & 1022 & 1016 & 241,41732 \\
\hline 01.09.2006 & 1016 & 1011 & 241,1869436 \\
\hline 02.09 .2006 & 1011 & 1008 & 240,714285 \\
\hline 03.09.2006 & 1008 & 1018 & 237,64243 \\
\hline 04.09.2006 & 1018 & 1024 & 238,593 \\
\hline 05.09 .2006 & 1024 & 1018 & 241,414538 \\
\hline 06.09.2006 & 1018 & 1011 & 241,661721 \\
\hline 07.09.2006 & 1011 & 1025 & 236,721951 \\
\hline 08.09.2006 & 1025 & 1030 & 238,834951 \\
\hline 09.09 .2006 & 1030 & 1024 & 241,4062 \\
\hline 10.09.2006 & 1024 & 1021 & 240,70519 \\
\hline 11.09.2006 & 1021 & 1018 & 240,7072692 \\
\hline 12.09 .2006 & 1018 & 1012 & 241,422924 \\
\hline 13.09 .2006 & 1012 & 1009 & 240,713577 \\
\hline 14.09 .2006 & 1009 & 1006 & 240,7157058 \\
\hline 15.09 .2006 & 1006 & 1009 & 239,286422 \\
\hline 16.09 .2006 & 1009 & 1006 & 240,715705 \\
\hline 17.09.2006 & 1006 & 1010 & 239,0495 \\
\hline 18.09.2006 & 1010 & 1013 & 239,28923 \\
\hline 19.09.2006 & 1013 & 1019 & 238,586849 \\
\hline 20.09 .2006 & 1019 & 1015 & 240,9458128 \\
\hline 21.09 .2006 & 1015 & 1012 & 240,711462 \\
\hline 22.09.2006 & 1012 & 1014 & 239,526627 \\
\hline 23.09.2006 & 1014 & 1010 & 240,95049 \\
\hline 24.09 .2006 & 1010 & 1006 & 240,95427 \\
\hline 25.09.2006 & 1006 & 1013 & 238,34155 \\
\hline 26.09.2006 & 1013 & 1016 & 239,29133 \\
\hline 27.09 .2006 & 1016 & 1012 & 240,9486166 \\
\hline 28.09.2006 & 1012 & 1009 & 240,71357 \\
\hline 29.09.2006 & 1009 & 1011 & 239,52522 \\
\hline
\end{tabular}

\begin{tabular}{|c|c|}
\hline$-0,476190476$ & \\
\hline$-0,238331678$ & \\
\hline$-0,47761194$ & \\
\hline$-0,478564307$ & \\
\hline$-0,72$ & \\
\hline 1,904761905 & 1,90476 \\
\hline$-0,71641791$ & \\
\hline$-0,718562874$ & \\
\hline 2,606120434 & 2,60612 \\
\hline 0,472906404 & 0,47291 \\
\hline$-0,236686391$ & \\
\hline$-0,4743083$ & \\
\hline 0,94488189 & 0,94488 \\
\hline$-1,904761905$ & \\
\hline$-0,71641791$ & \\
\hline 0,476663357 & 0,47666 \\
\hline 0,712871287 & 0,71287 \\
\hline$-2,642642643$ & \\
\hline 1,195219124 & 1,19522 \\
\hline 2,132280355 & 2,13228 \\
\hline 2,113502935 & 2,1135 \\
\hline$-1,417322835$ & \\
\hline$-1,18694362$ & \\
\hline$-0,714285714$ & \\
\hline 2,357563851 & 2,35756 \\
\hline 1,40625 & 1,40625 \\
\hline$-1,41453831$ & \\
\hline$-1,661721068$ & \\
\hline 3,27804878 & 3,27805 \\
\hline 1,165048544 & 1,16505 \\
\hline$-1,40625$ & \\
\hline$-0,705190989$ & \\
\hline$-0,707269155$ & \\
\hline$-1,422924901$ & \\
\hline$-0,7135778$ & \\
\hline$-0,715705765$ & \\
\hline 0,7135778 & 0,71358 \\
\hline$-0,715705765$ & \\
\hline 0,95049505 & 0,9505 \\
\hline 0,710760118 & 0,71076 \\
\hline 1,413150147 & 1,41315 \\
\hline$-0,945812808$ & \\
\hline$-0,711462451$ & \\
\hline 0,473372781 & 0,47337 \\
\hline$-0,95049505$ & \\
\hline$-0,954274354$ & \\
\hline 1,658440276 & 1,65844 \\
\hline 0,708661417 & 0,70866 \\
\hline$-0,948616601$ & \\
\hline$-0,7135778$ & \\
\hline 0,474777448 & 0,47478 \\
\hline
\end{tabular}




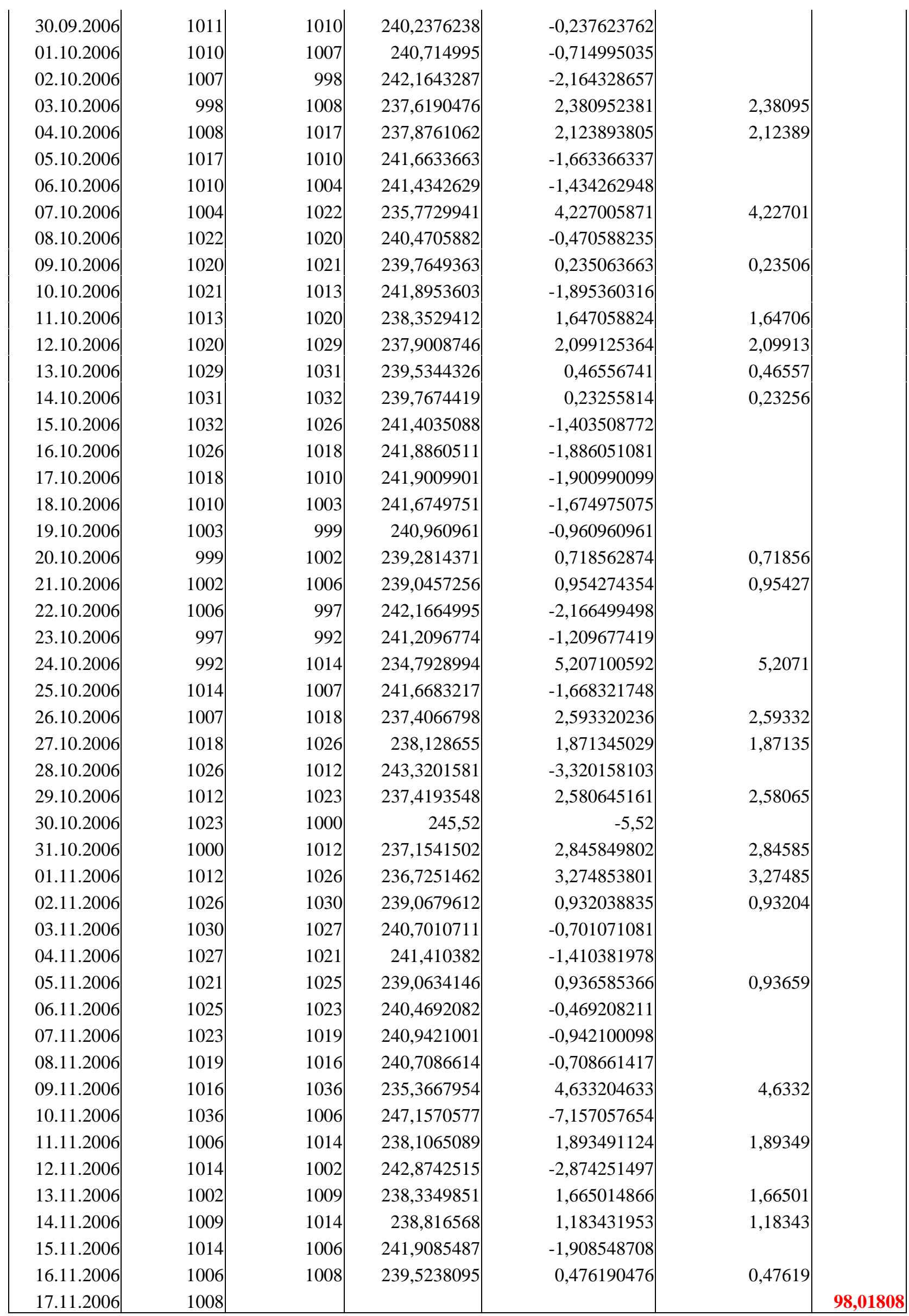




\begin{tabular}{|c|c|c|c|c|c|c|c|}
\hline Datum & $\begin{array}{c}\text { T1 } \\
\text { (max. } \\
\text { Temp.) }\end{array}$ & $\begin{array}{c}\text { T2 } \\
\text { (min. } \\
\text { Temp.) }\end{array}$ & T1 & $\mathbf{T} 2$ & $\begin{array}{c}240 *(1+0,0036 * \\
(\mathrm{T} 2-\mathrm{T} 1)) \\
=\mathrm{V} 2\end{array}$ & $\begin{array}{c}\text { Luftvolumenein- } \\
\text { strom } \\
\text { (=V1-V2) }\end{array}$ & $\begin{array}{c}\text { Summe } \\
\Delta \mathrm{V}\end{array}$ \\
\hline & $\left({ }^{\circ} \mathrm{C}\right)$ & $\left({ }^{\circ} \mathrm{C}\right)$ & (Kelvin) & (Kelvin) & (ml) & $(\mathrm{ml})$ & $(\mathrm{ml})$ \\
\hline 07.08.2006 & 25,2 & 14 & 298,2 & 287 & 230,3232 & 9,6768 & \\
\hline 08.08.2006 & 20,7 & 14,8 & 293,7 & 287,8 & 234,9024 & 5,0976 & \\
\hline 09.08.2006 & 20,7 & 13,8 & 293,7 & 286,8 & 234,0384 & 5,9616 & \\
\hline 10.08.2006 & 19,6 & 12,3 & 292,6 & 285,3 & 233,6928 & 6,3072 & \\
\hline 11.08.2006 & 16,7 & 10,7 & 289,7 & 283,7 & 234,816 & 5,184 & \\
\hline 12.08.2006 & 18,1 & 9,5 & 291,1 & 282,5 & 232,5696 & 7,4304 & \\
\hline 13.08.2006 & 20,6 & 6,9 & 293,6 & 279,9 & 228,1632 & 11,8368 & \\
\hline 14.08 .2006 & 19,8 & 8,9 & 292,8 & 281,9 & 230,5824 & 9,4176 & \\
\hline 15.08.2006 & 17,9 & 12,6 & 290,9 & 285,6 & 235,4208 & 4,5792 & \\
\hline 16.08 .2006 & 22,2 & 10,4 & 295,2 & 283,4 & 229,8048 & 10,1952 & \\
\hline 17.08.2006 & 24,3 & 12,1 & 297,3 & 285,1 & 229,4592 & 10,5408 & \\
\hline 18.08.2006 & 21,8 & 13,4 & 294,8 & 286,4 & 232,7424 & 7,2576 & \\
\hline 19.08.2006 & 26,8 & 11,7 & 299,8 & 284,7 & 226,9536 & 13,0464 & \\
\hline 20.08.2006 & 21,6 & 13,4 & 294,6 & 286,4 & 232,9152 & 7,0848 & \\
\hline 21.08.2006 & 18,3 & 13,4 & 291,3 & 286,4 & 235,7664 & 4,2336 & \\
\hline 22.08.2006 & 18,3 & 13,3 & 291,3 & 286,3 & 235,68 & 4,32 & \\
\hline 23.08.2006 & 20,8 & 13,3 & 293,8 & 286,3 & 233,52 & 6,48 & \\
\hline 24.08.2006 & 23,6 & 11,9 & 296,6 & 284,9 & 229,8912 & 10,1088 & \\
\hline 25.08.2006 & 18,4 & 11,7 & 291,4 & 284,7 & 234,2112 & 5,7888 & \\
\hline 26.08.2006 & 18,3 & 11,7 & 291,3 & 284,7 & 234,2976 & 5,7024 & \\
\hline 27.08.2006 & 19,6 & 11,7 & 292,6 & 284,7 & 233,1744 & 6,8256 & \\
\hline 28.08.2006 & 17,3 & 10,1 & 290,3 & 283,1 & 233,7792 & 6,2208 & \\
\hline 29.08.2006 & 16,6 & 10,1 & 289,6 & 283,1 & 234,384 & 5,616 & \\
\hline 30.08.2006 & 16,9 & 10,9 & 289,9 & 283,9 & 234,816 & 5,184 & \\
\hline 31.08 .2006 & 19,3 & 11,5 & 292,3 & 284,5 & 233,2608 & 6,7392 & \\
\hline 01.09 .2006 & 22,1 & 14,4 & 295,1 & 287,4 & 233,3472 & 6,6528 & \\
\hline 02.09.2006 & 25,3 & 13,9 & 298,3 & 286,9 & 230,1504 & 9,8496 & \\
\hline 03.09.2006 & 21,6 & 16,8 & 294,6 & 289,8 & 235,8528 & 4,1472 & \\
\hline 04.09 .2006 & 21,7 & 15,8 & 294,7 & 288,8 & 234,9024 & 5,0976 & \\
\hline 05.09 .2006 & 19,3 & 12,6 & 292,3 & 285,6 & 234,2112 & 5,7888 & \\
\hline 06.09.2006 & 25,9 & 16,2 & 298,9 & 289,2 & 231,6192 & 8,3808 & \\
\hline 07.09.2006 & 25,2 & 12,9 & 298,2 & 285,9 & 229,3728 & 10,6272 & \\
\hline 08.09.2006 & 19,2 & 8,7 & 292,2 & 281,7 & 230,928 & 9,072 & \\
\hline 09.09.2006 & 20,6 & 5,8 & 293,6 & 278,8 & 227,2128 & 12,7872 & \\
\hline 10.09.2006 & 24,7 & 7,3 & 297,7 & 280,3 & 224,9664 & 15,0336 & \\
\hline
\end{tabular}




\begin{tabular}{|r|c|r|r|r|}
\hline 11.09 .2006 & 26,9 & 8,9 & 299,9 & 281,9 \\
\hline 12.09 .2006 & 27,5 & 10,7 & 300,5 & 283,7 \\
\hline 13.09 .2006 & 28,2 & 10,4 & 301,2 & 283,4 \\
\hline 14.09 .2006 & 27,8 & 11,2 & 300,8 & 284,2 \\
\hline 15.09 .2006 & 25,3 & 12,3 & 298,3 & 285,3 \\
\hline 16.09 .2006 & 25,3 & 18,4 & 298,3 & 291,4 \\
\hline 17.09 .2006 & 24,8 & 12,6 & 297,8 & 285,6 \\
\hline 18.09 .2006 & 17,9 & 13,7 & 290,9 & 286,7 \\
\hline 19.09 .2006 & 20,8 & 13,6 & 293,8 & 286,6 \\
\hline 20.09 .2006 & 19,6 & 9,2 & 292,6 & 282,2 \\
\hline 21.09 .2006 & 25,1 & 6,2 & 298,1 & 279,2 \\
\hline 22.09 .2006 & 27,5 & 10,2 & 300,5 & 283,2 \\
\hline 23.09 .2006 & 26,6 & 10,2 & 299,6 & 283,2 \\
\hline 24.09 .2006 & 25,7 & 8,1 & 298,7 & 281,1 \\
\hline 25.09 .2006 & 24,3 & 7,6 & 297,3 & 280,6 \\
\hline 26.09 .2006 & 18,9 & 14,9 & 291,9 & 287,9 \\
\hline 27.09 .2006 & 20,6 & 11,5 & 293,6 & 284,5 \\
\hline 28.09 .2006 & 21,1 & 9,7 & 294,1 & 282,7 \\
\hline 29.09 .2006 & 22,4 & 10,3 & 295,4 & 283,3 \\
\hline 30.09 .2006 & 22,2 & 11,4 & 295,2 & 284,4 \\
\hline 01.10 .2006 & 20,7 & 10,6 & 293,7 & 283,6 \\
\hline 02.10 .2006 & 16,5 & 12,9 & 289,5 & 285,9 \\
\hline 03.10 .2006 & 15,9 & 11,9 & 288,9 & 284,9 \\
\hline 04.10 .2006 & 13,8 & 10,4 & 286,8 & 283,4 \\
\hline 05.10 .2006 & 15,1 & 9,5 & 288,1 & 282,5 \\
\hline 06.10 .2006 & 15,9 & 11,7 & 288,9 & 284,7 \\
\hline 07.10 .2006 & 14,6 & 9,8 & 287,6 & 282,8 \\
\hline 08.10 .2006 & 15,7 & 6,3 & 288,7 & 279,3 \\
\hline 09.10 .2006 & 18,7 & 3,7 & 291,7 & 276,7 \\
\hline 10.10 .2006 & 20,8 & 8,6 & 293,8 & 281,6 \\
\hline 11.10 .2006 & 19,3 & 6,5 & 292,3 & 279,5 \\
\hline 12.10 .2006 & 21,5 & 6,1 & 294,5 & 279,1 \\
\hline 13.10 .2006 & 16,3 & 12,6 & 289,3 & 285,6 \\
\hline 14.10 .2006 & 13,4 & 11,2 & 286,4 & 284,2 \\
\hline 15.10 .2006 & 12,8 & 6,9 & 285,8 & 279,9 \\
\hline 16.10 .2006 & 16 & 5,9 & 289 & 278,9 \\
\hline 17.10 .2006 & 16,2 & 2,8 & 289,2 & 275,8 \\
\hline 18.10 .2006 & 13,9 & 2,1 & 286,9 & 275,1 \\
\hline 19.10 .2006 & 17,7 & 5,9 & 290,7 & 278,9 \\
\hline 20.10 .2006 & 17,3 & 10,7 & 290,3 & 283,7 \\
\hline 21.10 .2006 & 17,2 & 11,2 & 290,2 & 284,2 \\
\hline 22.10 .2006 & 16,4 & 12,3 & 289,4 & 285,3 \\
\hline 23.10 .2006 & 17,7 & 13,7 & 290,7 & 286,7 \\
\hline 24.10 .2006 & 16,7 & 11,7 & 289,7 & 284,7 \\
\hline 25.10 .2006 & 15,3 & 7,6 & 288,3 & 280,6 \\
\hline 26.10 .2006 & 21,4 & 10,2 & 294,4 & 283,2 \\
\hline 27.10 .2006 & 18,2 & 11,2 & 291,2 & 284,2 \\
\hline 28.10 .2006 & 14 & 6,8 & 287 & 279,8 \\
\hline 29.10 .2006 & 16 & 10,3 & 289 & 283,3 \\
\hline 30.10 .2006 & 12,4 & 6,7 & 285,4 & 279,7 \\
\hline 31.10 .2006 & 13,1 & 9,4 & 286,1 & 282,4 \\
\hline & & & & \\
\hline
\end{tabular}

224,448
225,4848
224,6208
225,657
228,768

228,768

234,0384

229,4592

236,3712

233,7792

231,0144

223,6704

225,0528

225,8304

224,7936

225,5712

236,544

232,1376

230,1504

229,5456

230,6688

231,2736

236,8896

236,544

237,0624

235,1616

236,3712

235,8528

231,8784

227,04

229,4592

228,9408

226,6944

236,8032

238,0992

234,9024

231,2736

228,4224

229,8048

229,8048

234,2976

234,816

236,4576

236,544

235,68

233,3472

230,3232

233,952

233,7792

235,0752

235,0752

236,8032

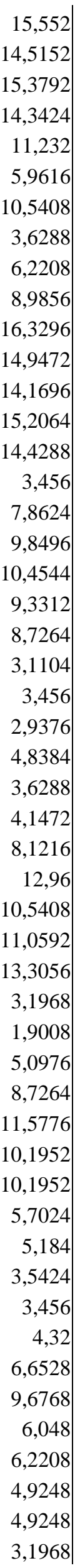

14,5152

15,3792

11,232

5,9616

0,5408

3,6288

6,2208

8,9856

16,3296

14,9472

15,2064

14,4288

7,8624

9,8496

0,4544

9,3312

8,7264

3,1104

3,456

2,9376

4,8384

3,6288

4,1472

,1216

1,0592

3,3056

3,1968

1,9008

5,0976

8,7264

11,5776

10,1952

,1952

5,7024

5,184

3,5424

3,456

6528

6,048

6,2208

4,9248

4,9248

3,1968 


\begin{tabular}{|c|c|c|c|c|c|c|}
\hline 01.11 .2006 & 9,3 & 0,2 & 282,3 & 273,2 & 232,1376 & 7,8624 \\
\hline 02.11.2006 & 3,9 & $-1,9$ & 276,9 & 271,1 & 234,9888 & 5,0112 \\
\hline 03.11 .2006 & 7,1 & -1 & 280,1 & 272 & 233,0016 & 6,9984 \\
\hline 04.11 .2006 & 9,2 & 3,6 & 282,2 & 276,6 & 235,1616 & 4,8384 \\
\hline 05.11 .2006 & 10,5 & 8,1 & 283,5 & 281,1 & 237,9264 & 2,0736 \\
\hline 06.11 .2006 & 11,5 & 9,4 & 284,5 & 282,4 & 238,1856 & 1,8144 \\
\hline 07.11 .2006 & 10,2 & 4,7 & 283,2 & 277,7 & 235,248 & 4,752 \\
\hline 08.11 .2006 & 11,2 & 3,6 & 284,2 & 276,6 & 233,4336 & 6,5664 \\
\hline 09.11 .2006 & 10,9 & 5,8 & 283,9 & 278,8 & 235,5936 & 4,4064 \\
\hline 10.11 .2006 & 8,7 & 3,4 & 281,7 & 276,4 & 235,4208 & 4,5792 \\
\hline 11.11.2006 & 9,2 & 3,4 & 282,2 & 276,4 & 234,9888 & 5,0112 \\
\hline 12.11.2006 & 7,4 & 3,6 & 280,4 & 276,6 & 236,7168 & 3,2832 \\
\hline 13.11.2006 & 11,9 & 5,1 & 284,9 & 278,1 & 234,1248 & 5,8752 \\
\hline 14.11.2006 & 13,6 & 7,4 & 286,6 & 280,4 & 234,6432 & 5,3568 \\
\hline 15.11.2006 & 14,6 & 7,6 & 287,6 & 280,6 & 233,952 & 6,048 \\
\hline 16.11.2006 & 16,9 & 8 & 289,9 & 281 & 232,3104 & 7,6896 \\
\hline 17.11.2006 & 15,1 & 8,3 & 288,1 & 281,3 & 234,1248 & 5,8752 \\
\hline 18.11.2006 & 12,6 & 3,3 & 285,6 & 276,3 & 231,9648 & 8,0352 \\
\hline 19.11.2006 & 10 & 2,7 & 283 & 275,7 & 233,6928 & 6,3072 \\
\hline 20.11.2006 & 7,4 & 2,2 & 280,4 & 275,2 & 235,5072 & 4,4928 \\
\hline 21.11.2006 & 9,6 & 3,3 & 282,6 & 276,3 & 234,5568 & 5,4432 \\
\hline 22.11.2006 & 6,2 & 0,8 & 279,2 & 273,8 & 235,3344 & 4,6656 \\
\hline 23.11.2006 & 12,8 & 4,6 & 285,8 & 277,6 & 232,9152 & 7,0848 \\
\hline 24.11.2006 & 11,8 & 6,8 & 284,8 & 279,8 & 235,68 & 4,32 \\
\hline 25.11.2006 & 15,4 & 11,1 & 288,4 & 284,1 & 236,2848 & 3,7152 \\
\hline 26.11.2006 & 13,1 & 5 & 286,1 & 278 & 233,0016 & 6,9984 \\
\hline 27.11.2006 & 12,1 & 1,8 & 285,1 & 274,8 & 231,1008 & 8,8992 \\
\hline 28.11 .2006 & 8,6 & 3,2 & 281,6 & 276,2 & 235,3344 & 4,6656 \\
\hline 29.11.2006 & 9,5 & 2,7 & 282,5 & 275,7 & 234,1248 & 5,8752 \\
\hline 30.11 .2006 & 7,3 & 0,7 & 280,3 & 273,7 & 234,2976 & 5,7024 \\
\hline 01.12 .2006 & 9,3 & 1,2 & 282,3 & 274,2 & 233,0016 & 6,9984 \\
\hline 02.12.2006 & 9,7 & 3,1 & 282,7 & 276,1 & 234,2976 & 5,7024 \\
\hline 03.12.2006 & 12,7 & 6,6 & 285,7 & 279,6 & 234,7296 & 5,2704 \\
\hline \multirow[t]{2}{*}{04.12 .2006} & 9,5 & 5,8 & 282,5 & 278,8 & 236,8032 & 3,1968 \\
\hline & & & & & & \\
\hline
\end{tabular}


Obernjesa (Kornhaus)

Lagerungszeitraum: 07.08.06-

23.11.06

\begin{tabular}{|c|c|c|c|c|c|c|c|}
\hline Datum & $\begin{array}{c}\text { T1 } \\
\text { (max. } \\
\text { Temp.) }\end{array}$ & $\begin{array}{c}\text { T2 } \\
\text { (min. } \\
\text { Temp.) }\end{array}$ & T1 & $\mathbf{T} 2$ & $\begin{array}{c}240 *(1+0,0036 \\
*(\mathrm{~T} 2-\mathrm{T} 1)) \\
=\mathrm{V} 2\end{array}$ & $\begin{array}{c}\text { Luftvolumenein } \\
\text { strom } \\
(=\mathrm{V} 1-\mathrm{V} 2)\end{array}$ & $\begin{array}{c}\text { Summe } \\
\Delta \mathrm{V}\end{array}$ \\
\hline & $\left({ }^{\circ} \mathrm{C}\right)$ & $\left({ }^{\circ} \mathrm{C}\right)$ & (Kelvin) & (Kelvin) & (ml) & (ml) & $(\mathrm{ml})$ \\
\hline 07.08 .2006 & 25,2 & 14 & 298,2 & 287 & 230,3232 & 9,6768 & \\
\hline 08.08.2006 & 20,7 & 14,8 & 293,7 & 287,8 & 234,9024 & 5,0976 & \\
\hline 09.08.2006 & 20,7 & 13,8 & 293,7 & 286,8 & 234,0384 & 5,9616 & \\
\hline 10.08 .2006 & 19,6 & 12,3 & 292,6 & 285,3 & 233,6928 & 6,3072 & \\
\hline 11.08 .2006 & 16,7 & 10,7 & 289,7 & 283,7 & 234,816 & 5,184 & \\
\hline 12.08 .2006 & 18,1 & 9,5 & 291,1 & 282,5 & 232,5696 & 7,4304 & \\
\hline 13.08.2006 & 20,6 & 6,9 & 293,6 & 279,9 & 228,1632 & 11,8368 & \\
\hline 14.08 .2006 & 19,8 & 8,9 & 292,8 & 281,9 & 230,5824 & 9,4176 & \\
\hline 15.08 .2006 & 17,9 & 12,6 & 290,9 & 285,6 & 235,4208 & 4,5792 & \\
\hline 16.08 .2006 & 22,2 & 10,4 & 295,2 & 283,4 & 229,8048 & 10,1952 & \\
\hline 17.08 .2006 & 24,3 & 12,1 & 297,3 & 285,1 & 229,4592 & 10,5408 & \\
\hline 18.08.2006 & 21,8 & 13,4 & 294,8 & 286,4 & 232,7424 & 7,2576 & \\
\hline 19.08.2006 & 26,8 & 11,7 & 299,8 & 284,7 & 226,9536 & 13,0464 & \\
\hline 20.08.2006 & 21,6 & 13,4 & 294,6 & 286,4 & 232,9152 & 7,0848 & \\
\hline 21.08.2006 & 18,3 & 13,4 & 291,3 & 286,4 & 235,7664 & 4,2336 & \\
\hline 22.08 .2006 & 18,3 & 13,3 & 291,3 & 286,3 & 235,68 & 4,32 & \\
\hline 23.08 .2006 & 20,8 & 13,3 & 293,8 & 286,3 & 233,52 & 6,48 & \\
\hline 24.08 .2006 & 23,6 & 11,9 & 296,6 & 284,9 & 229,8912 & 10,1088 & \\
\hline 25.08 .2006 & 18,4 & 11,7 & 291,4 & 284,7 & 234,2112 & 5,7888 & \\
\hline 26.08.2006 & 18,3 & 11,7 & 291,3 & 284,7 & 234,2976 & 5,7024 & \\
\hline 27.08.2006 & 19,6 & 11,7 & 292,6 & 284,7 & 233,1744 & 6,8256 & \\
\hline 28.08.2006 & 17,3 & 10,1 & 290,3 & 283,1 & 233,7792 & 6,2208 & \\
\hline 29.08.2006 & 16,6 & 10,1 & 289,6 & 283,1 & 234,384 & 5,616 & \\
\hline 30.08 .2006 & 16,9 & 10,9 & 289,9 & 283,9 & 234,816 & 5,184 & \\
\hline 31.08 .2006 & 19,3 & 11,5 & 292,3 & 284,5 & 233,2608 & 6,7392 & \\
\hline 01.09 .2006 & 22,1 & 14,4 & 295,1 & 287,4 & 233,3472 & 6,6528 & \\
\hline 02.09 .2006 & 25,3 & 13,9 & 298,3 & 286,9 & 230,1504 & 9,8496 & \\
\hline 03.09 .2006 & 21,6 & 16,8 & 294,6 & 289,8 & 235,8528 & 4,1472 & \\
\hline 04.09 .2006 & 21,7 & 15,8 & 294,7 & 288,8 & 234,9024 & 5,0976 & \\
\hline 05.09 .2006 & 19,3 & 12,6 & 292,3 & 285,6 & 234,2112 & 5,7888 & \\
\hline 06.09 .2006 & 25,9 & 16,2 & 298,9 & 289,2 & 231,6192 & 8,3808 & \\
\hline 07.09 .2006 & 25,2 & 12,9 & 298,2 & 285,9 & 229,3728 & 10,6272 & \\
\hline 08.09 .2006 & 19,2 & 8,7 & 292,2 & 281,7 & 230,928 & 9,072 & \\
\hline 09.09 .2006 & 20,6 & 5,8 & 293,6 & 278,8 & 227,2128 & 12,7872 & \\
\hline 10.09 .2006 & 24,7 & 7,3 & 297,7 & 280,3 & 224,9664 & 15,0336 & \\
\hline 11.09 .2006 & 26,9 & 8,9 & 299,9 & 281,9 & 224,448 & 15,552 & \\
\hline 12.09 .2006 & 27,5 & 10,7 & 300,5 & 283,7 & 225,4848 & 14,5152 & \\
\hline 13.09 .2006 & 28,2 & 10,4 & 301,2 & 283,4 & 224,6208 & 15,3792 & \\
\hline 14.09 .2006 & 27,8 & 11,2 & 300,8 & 284,2 & 225,6576 & 14,3424 & \\
\hline 15.09 .2006 & 25,3 & 12,3 & 298,3 & 285,3 & 228,768 & 11,232 & \\
\hline
\end{tabular}




\begin{tabular}{|c|c|c|c|c|c|c|}
\hline 16.09.2006 & 25,3 & 18,4 & 298,3 & 291,4 & 234,0384 & 5,9616 \\
\hline 17.09.2006 & 24,8 & 12,6 & 297,8 & 285,6 & 229,4592 & 10,5408 \\
\hline 18.09.2006 & 17,9 & 13,7 & 290,9 & 286,7 & 236,3712 & 3,6288 \\
\hline 19.09.2006 & 20,8 & 13,6 & 293,8 & 286,6 & 233,7792 & 6,220 \\
\hline 20.09.2006 & 19,6 & 9,2 & 292,6 & 282,2 & 231,0144 & 8,985 \\
\hline 21.09.2006 & 25,1 & 6,2 & 298,1 & 279,2 & 223,6704 & 16,329 \\
\hline 22.09 .2006 & 27,5 & 10,2 & 300,5 & 283,2 & 225,0528 & 14,947 \\
\hline 23.09 .2006 & 26,6 & 10,2 & 299,6 & 283,2 & 225,8304 & 14,16 \\
\hline 24.09 .2006 & 25,7 & 8,1 & 298,7 & 281,1 & 224,7936 & 15,20 \\
\hline 25.09 .2006 & 24,3 & 7,6 & 297,3 & 280,6 & 225,5712 & 14,428 \\
\hline 26.09 .2006 & 18,9 & 14,9 & 291,9 & 287,9 & 236,544 & 3,45 \\
\hline 27.09 .2006 & 20,6 & 11,5 & 293,6 & 284,5 & 232,1376 & 7,862 \\
\hline 28.09 .2006 & 21,1 & 9,7 & 294,1 & 282,7 & 230,1504 & 9,84 \\
\hline 29.09 .2006 & 22,4 & 10,3 & 295,4 & 283,3 & 229,5456 & 10,45 \\
\hline 30.09.2006 & 22,2 & 11,4 & 295,2 & 284,4 & 230,6688 & 9,3312 \\
\hline 01.10 .2006 & 20,7 & 10,6 & 293,7 & 283,6 & 231,2736 & 8,726 \\
\hline 02.10 .2006 & 16,5 & 12,9 & 289,5 & 285,9 & 236,8896 & 3,110 \\
\hline 03.10 .2006 & 15,9 & 11,9 & 288,9 & 284,9 & 236,544 & 3,4 \\
\hline 04.10 .2006 & 13,8 & 10,4 & 286,8 & 283,4 & 237,0624 & 2,937 \\
\hline 05.10 .2006 & 15,1 & 9,5 & 288,1 & 282,5 & 235,1616 & 4,83 \\
\hline 06.10 .2006 & 15,9 & 11,7 & 288,9 & 284,7 & 236,3712 & 3,628 \\
\hline 07.10 .2006 & 14,6 & 9,8 & 287,6 & 282,8 & 235,8528 & 4,1472 \\
\hline 08.10 .2006 & 15,7 & 6,3 & 288,7 & 279,3 & 231,8784 & 8,121 \\
\hline 09.10.2006 & 18,7 & 3,7 & 291,7 & 276,7 & 227,04 & 12 \\
\hline 10.10.2006 & 20,8 & 8,6 & 293,8 & 281,6 & 229,4592 & 10,5408 \\
\hline 11.10 .2006 & 19,3 & 6,5 & 292,3 & 279,5 & 228,9408 & 11,0592 \\
\hline 12.10 .2006 & 21,5 & 6,1 & 294,5 & 279,1 & 226,6944 & 13,305 \\
\hline 13.10.2006 & 16,3 & 12,6 & 289,3 & 285,6 & 236,8032 & 3,1968 \\
\hline 14.10.2006 & 13,4 & 11,2 & 286,4 & 284,2 & 238,0992 & 1,900 \\
\hline 15.10 .2006 & 12,8 & 6,9 & 285,8 & 279,9 & 234,9024 & 5,097 \\
\hline 16.10 .2006 & 16 & 5,9 & 289 & 278,9 & 231,2736 & 8,7264 \\
\hline 17.10.2006 & 16,2 & 2,8 & 289,2 & 275,8 & 228,4224 & 11,5776 \\
\hline 18.10.2006 & 13,9 & 2,1 & 286,9 & 275,1 & 229,8048 & 10,1952 \\
\hline 19.10.2006 & 17,7 & 5,9 & 290,7 & 278,9 & 229,8048 & 10,1952 \\
\hline 20.10 .2006 & 17,3 & 10,7 & 290,3 & 283,7 & 234,2976 & 5,7024 \\
\hline 21.10 .2006 & 17,2 & 11,2 & 290,2 & 284,2 & 234,816 & 5,18 \\
\hline 22.10 .2006 & 16,4 & 12,3 & 289,4 & 285,3 & 236,4576 & 3,542 \\
\hline 23.10 .2006 & 17,7 & 13,7 & 290,7 & 286,7 & 236,544 & 3,456 \\
\hline 24.10 .2006 & 16,7 & 11,7 & 289,7 & 284,7 & 235,68 & 4,32 \\
\hline 25.10 .2006 & 15,3 & 7,6 & 288,3 & 280,6 & 233,3472 & 6,652 \\
\hline 26.10 .2006 & 21,4 & 10,2 & 294,4 & 283,2 & 230,3232 & 9,6768 \\
\hline 27.10 .2006 & 18,2 & 11,2 & 291,2 & 284,2 & 233,952 & 6,048 \\
\hline 28.10 .2006 & 14 & 6,8 & 287 & 279,8 & 233,7792 & 6,2208 \\
\hline 29.10.2006 & 16 & 10,3 & 289 & 283,3 & 235,0752 & 4,9248 \\
\hline 30.10 .2006 & 12,4 & 6,7 & 285,4 & 279,7 & 235,0752 & 4,924 \\
\hline 31.10 .2006 & 13,1 & 9,4 & 286,1 & 282,4 & 236,8032 & 3,1968 \\
\hline 01.11 .2006 & 9,3 & 0,2 & 282,3 & 273,2 & 232,1376 & 7,8624 \\
\hline 02.11.2006 & 3,9 & $-1,9$ & 276,9 & 271,1 & 234,9888 & 5,0112 \\
\hline 03.11.2006 & 7,1 & -1 & 280,1 & 272 & 233,0016 & 6,99 \\
\hline 04.11 .2006 & 9,2 & 3,6 & 282,2 & 276,6 & 235,1616 & 4,83 \\
\hline 05.11 .2006 & 10,5 & 8,1 & 283,5 & 281,1 & 237,9264 & 2,07 \\
\hline
\end{tabular}




\begin{tabular}{|c|c|c|c|c|c|c|c|}
\hline 06.11 .2006 & 11,5 & 9,4 & 284,5 & 282,4 & 238,1856 & 1,8144 & \\
\hline 07.11 .2006 & 10,2 & 4,7 & 283,2 & 277,7 & 235,248 & 4,752 & \\
\hline 08.11 .2006 & 11,2 & 3,6 & 284,2 & 276,6 & 233,4336 & 6,5664 & \\
\hline 09.11 .2006 & 10,9 & 5,8 & 283,9 & 278,8 & 235,5936 & 4,4064 & \\
\hline 10.11 .2006 & 8,7 & 3,4 & 281,7 & 276,4 & 235,4208 & 4,5792 & \\
\hline 11.11.2006 & 9,2 & 3,4 & 282,2 & 276,4 & 234,9888 & 5,0112 & \\
\hline 12.11.2006 & 7,4 & 3,6 & 280,4 & 276,6 & 236,7168 & 3,2832 & \\
\hline 13.11.2006 & 11,9 & 5,1 & 284,9 & 278,1 & 234,1248 & 5,8752 & \\
\hline 14.11.2006 & 13,6 & 7,4 & 286,6 & 280,4 & 234,6432 & 5,3568 & \\
\hline 15.11.2006 & 14,6 & 7,6 & 287,6 & 280,6 & 233,952 & 6,048 & \\
\hline 16.11.2006 & 16,9 & 8 & 289,9 & 281 & 232,3104 & 7,6896 & \\
\hline 17.11.2006 & 15,1 & 8,3 & 288,1 & 281,3 & 234,1248 & 5,8752 & \\
\hline 18.11.2006 & 12,6 & 3,3 & 285,6 & 276,3 & 231,9648 & 8,0352 & \\
\hline 19.11.2006 & 10 & 2,7 & 283 & 275,7 & 233,6928 & 6,3072 & \\
\hline 20.11.2006 & 7,4 & 2,2 & 280,4 & 275,2 & 235,5072 & 4,4928 & \\
\hline 21.11.2006 & 9,6 & 3,3 & 282,6 & 276,3 & 234,5568 & 5,4432 & \\
\hline 22.11.2006 & 6,2 & 0,8 & 279,2 & 273,8 & 235,3344 & 4,6656 & \\
\hline \multirow[t]{2}{*}{ 23.11.2006 } & 12,8 & 4,6 & 285,8 & 277,6 & 232,9152 & 7,0848 & \\
\hline & & & & & & & 816,3072 \\
\hline
\end{tabular}

Scharzfeld (Kornhaus)

Lagerungszeitraum: 07.08.06 23.11.06

\begin{tabular}{|c|c|c|c|c|c|c|c|}
\hline Datum & \begin{tabular}{|c} 
T1 \\
(max. \\
Temp. $)$ \\
$\left({ }^{\circ} \mathrm{C}\right)$ \\
\end{tabular} & $\begin{array}{c}\text { T2 } \\
\text { (min. } \\
\text { Temp.) } \\
\left({ }^{\circ} \mathrm{C}\right)\end{array}$ & (Kelvin) & $\begin{array}{c}\text { T2 } \\
\text { (Kelvin) }\end{array}$ & $\begin{array}{c}240 *(1+0,0036 * \\
(\mathrm{T} 2-\mathrm{T} 1)) \\
=\mathrm{V} 2 \\
\\
(\mathrm{ml})\end{array}$ & $\begin{array}{c}\text { Luftvolumenein- } \\
\text { strom (=V1-V2) } \\
\text { (ml) }\end{array}$ & $\begin{array}{c}\text { Summe } \\
\Delta \mathbf{V} \\
\\
\text { (ml) } \\
\end{array}$ \\
\hline 07.08.2006 & 25,2 & 14 & 298,2 & 287 & 230,3232 & 9,6768 & \\
\hline 08.08.2006 & 20,7 & 14,8 & 293,7 & 287,8 & 234,9024 & 5,0976 & \\
\hline 09.08.2006 & 20,7 & 13,8 & 293,7 & 286,8 & 234,0384 & 5,9616 & \\
\hline 10.08 .2006 & 19,6 & 12,3 & 292,6 & 285,3 & 233,6928 & 6,3072 & \\
\hline 11.08 .2006 & 16,7 & 10,7 & 289,7 & 283,7 & 234,816 & 5,184 & \\
\hline 12.08 .2006 & 18,1 & 9,5 & 291,1 & 282,5 & 232,5696 & 7,4304 & \\
\hline 13.08 .2006 & 20,6 & 6,9 & 293,6 & 279,9 & 228,1632 & 11,8368 & \\
\hline 14.08 .2006 & 19,8 & 8,9 & 292,8 & 281,9 & 230,5824 & 9,4176 & \\
\hline 15.08 .2006 & 17,9 & 12,6 & 290,9 & 285,6 & 235,4208 & 4,5792 & \\
\hline 16.08 .2006 & 22,2 & 10,4 & 295,2 & 283,4 & 229,8048 & 10,1952 & \\
\hline 17.08.2006 & 24,3 & 12,1 & 297,3 & 285,1 & 229,4592 & 10,5408 & \\
\hline 18.08.2006 & 21,8 & 13,4 & 294,8 & 286,4 & 232,7424 & 7,2576 & \\
\hline 19.08.2006 & 26,8 & 11,7 & 299,8 & 284,7 & 226,9536 & 13,0464 & \\
\hline 20.08 .2006 & 21,6 & 13,4 & 294,6 & 286,4 & 232,9152 & 7,0848 & \\
\hline 21.08.2006 & 18,3 & 13,4 & 291,3 & 286,4 & 235,7664 & 4,2336 & \\
\hline 22.08 .2006 & 18,3 & 13,3 & 291,3 & 286,3 & 235,68 & 4,32 & \\
\hline 23.08.2006 & 20,8 & 13,3 & 293,8 & 286,3 & 233,52 & 6,48 & \\
\hline
\end{tabular}




\begin{tabular}{|c|c|}
\hline 24.08.2006 & 23,6 \\
\hline 25.08.2006 & 18,4 \\
\hline 26.08.2006 & 18,3 \\
\hline 27.08.2006 & 19,6 \\
\hline 28.08.2006 & 17,3 \\
\hline 29.08 .2006 & 16,6 \\
\hline 30.08.2006 & 16,9 \\
\hline 31.08 .2006 & 19,3 \\
\hline 01.09.2006 & 22,1 \\
\hline 02.09.2006 & 25,3 \\
\hline 03.09.2006 & 21,6 \\
\hline 04.09.2006 & 21,7 \\
\hline 05.09.2006 & 19,3 \\
\hline 06.09 .2006 & 25,9 \\
\hline 07.09.2006 & 25,2 \\
\hline 08.09 .2006 & 19,2 \\
\hline 09.09.2006 & 20,6 \\
\hline 10.09 .2006 & 24,7 \\
\hline 11.09 .2006 & 26,9 \\
\hline 12.09.2006 & 27,5 \\
\hline 13.09 .2006 & 28,2 \\
\hline 14.09 .2006 & 27,8 \\
\hline 15.09.2006 & 25,3 \\
\hline 16.09 .2006 & 25,3 \\
\hline 17.09 .2006 & 24,8 \\
\hline 18.09 .2006 & 17,9 \\
\hline 19.09 .2006 & 20,8 \\
\hline 20.09 .2006 & 19,6 \\
\hline 21.09 .2006 & 25,1 \\
\hline 22.09 .2006 & 27,5 \\
\hline 23.09.2006 & 26,6 \\
\hline 24.09.2006 & 25,7 \\
\hline 25.09 .2006 & 24,3 \\
\hline 26.09.2006 & 18,9 \\
\hline 27.09.2006 & 20,6 \\
\hline 28.09 .2006 & 21,1 \\
\hline 29.09 .2006 & 22,4 \\
\hline 30.09 .2006 & 22,2 \\
\hline 01.10 .2006 & 20,7 \\
\hline 02.10 .2006 & 16,5 \\
\hline 03.10 .2006 & 15,9 \\
\hline 04.10 .2006 & 13,8 \\
\hline 05.10 .2006 & 15,1 \\
\hline 06.10 .2006 & 15,9 \\
\hline 07.10 .2006 & 14,6 \\
\hline 08.10 .2006 & 15,7 \\
\hline 09.10 .2006 & 18,7 \\
\hline 10.10 .2006 & 20,8 \\
\hline 11.10 .2006 & 19,3 \\
\hline 12.10 .200 & 21,5 \\
\hline 13.10 .200 & 16,3 \\
\hline
\end{tabular}

\begin{tabular}{|c|c|c|}
\hline 11,9 & 296,6 & 284,9 \\
\hline 11,7 & 291,4 & 284,7 \\
\hline 11,7 & 291,3 & 284,7 \\
\hline 11,7 & 292,6 & 284,7 \\
\hline 10,1 & 290,3 & 283,1 \\
\hline 10,1 & 289,6 & 283,1 \\
\hline 10,9 & 289,9 & 283,9 \\
\hline 11,5 & 292,3 & 284,5 \\
\hline 14,4 & 295,1 & 287,4 \\
\hline 13,9 & 298,3 & 286,9 \\
\hline 16,8 & 294,6 & 289,8 \\
\hline 15,8 & 294,7 & 288,8 \\
\hline 12,6 & 292,3 & 285,6 \\
\hline 16,2 & 298,9 & 289,2 \\
\hline 12,9 & 298,2 & 285,9 \\
\hline 8,7 & 292,2 & 281,7 \\
\hline 5,8 & 293,6 & 278,8 \\
\hline 7,3 & 297,7 & 280,3 \\
\hline 8,9 & 299,9 & 281,9 \\
\hline 10,7 & 300,5 & 283,7 \\
\hline 10,4 & 301,2 & 283,4 \\
\hline 11,2 & 300,8 & 284,2 \\
\hline 12,3 & 298,3 & 285,3 \\
\hline 18,4 & 298,3 & 291,4 \\
\hline 12,6 & 297,8 & 285,6 \\
\hline 13,7 & 290,9 & 286,7 \\
\hline 13,6 & 293,8 & 286,6 \\
\hline 9,2 & 292,6 & 282,2 \\
\hline 6,2 & 298,1 & 279,2 \\
\hline 10,2 & 300,5 & 283,2 \\
\hline 10,2 & 299,6 & 283,2 \\
\hline 8,1 & 298,7 & 281,1 \\
\hline 7,6 & 297,3 & 280,6 \\
\hline 14,9 & 291,9 & 287,9 \\
\hline 11,5 & 293,6 & 284,5 \\
\hline 9,7 & 294,1 & 282,7 \\
\hline 10,3 & 295,4 & 283,3 \\
\hline 11,4 & 295,2 & 284,4 \\
\hline 10,6 & 293,7 & 283,6 \\
\hline 12,9 & 289,5 & 285,9 \\
\hline 11,9 & 288,9 & 284,9 \\
\hline 10,4 & 286,8 & 283,4 \\
\hline 9,5 & 288,1 & 282,5 \\
\hline 11,7 & 288,9 & 284,7 \\
\hline 9,8 & 287,6 & 282,8 \\
\hline 6,3 & 288,7 & 279,3 \\
\hline 3,7 & 291,7 & 276,7 \\
\hline 8,6 & 293,8 & 281,6 \\
\hline 6,5 & 292,3 & 279,5 \\
\hline 6,1 & 294,5 & 279,1 \\
\hline 12,6 & 289,3 & 285,6 \\
\hline
\end{tabular}

$\begin{array}{r}229,8912 \\ 234,2112 \\ 234,2976 \\ 233,1744 \\ 233,7792 \\ 234,384 \\ 234,816 \\ 233,2608 \\ 233,3472 \\ 230,1504 \\ 235,8528 \\ 234,9024 \\ 234,2112 \\ 231,6192 \\ 229,3728 \\ 230,928 \\ \hline 227,218\end{array}$

10,1088 


\begin{tabular}{|c|c|c|c|c|c|c|c|}
\hline 14.10 .2006 & 13,4 & 11,2 & 286,4 & 284,2 & 238,0992 & 1,9008 & \\
\hline 15.10.2006 & 12,8 & 6,9 & 285,8 & 279,9 & 234,9024 & 5,0976 & \\
\hline 16.10.2006 & 16 & 5,9 & 289 & 278,9 & 231,2736 & 8,7264 & \\
\hline 17.10.2006 & 16,2 & 2,8 & 289,2 & 275,8 & 228,4224 & 11,5776 & \\
\hline 18.10.2006 & 13,9 & 2,1 & 286,9 & 275,1 & 229,8048 & 10,1952 & \\
\hline 19.10.2006 & 17,7 & 5,9 & 290,7 & 278,9 & 229,8048 & 10,1952 & \\
\hline 20.10 .2006 & 17,3 & 10,7 & 290,3 & 283,7 & 234,2976 & 5,7024 & \\
\hline 21.10 .2006 & 17,2 & 11,2 & 290,2 & 284,2 & 234,816 & 5,184 & \\
\hline 22.10 .2006 & 16,4 & 12,3 & 289,4 & 285,3 & 236,4576 & 3,5424 & \\
\hline 23.10.2006 & 17,7 & 13,7 & 290,7 & 286,7 & 236,544 & 3,456 & \\
\hline 24.10 .2006 & 16,7 & 11,7 & 289,7 & 284,7 & 235,68 & 4,32 & \\
\hline 25.10 .2006 & 15,3 & 7,6 & 288,3 & 280,6 & 233,3472 & 6,6528 & \\
\hline 26.10 .2006 & 21,4 & 10,2 & 294,4 & 283,2 & 230,3232 & 9,6768 & \\
\hline 27.10 .2006 & 18,2 & 11,2 & 291,2 & 284,2 & 233,952 & 6,048 & \\
\hline 28.10 .2006 & 14 & 6,8 & 287 & 279,8 & 233,7792 & 6,2208 & \\
\hline 29.10.2006 & 16 & 10,3 & 289 & 283,3 & 235,0752 & 4,9248 & \\
\hline 30.10 .2006 & 12,4 & 6,7 & 285,4 & 279,7 & 235,0752 & 4,9248 & \\
\hline 31.10 .2006 & 13,1 & 9,4 & 286,1 & 282,4 & 236,8032 & 3,1968 & \\
\hline 01.11 .2006 & 9,3 & 0,2 & 282,3 & 273,2 & 232,1376 & 7,8624 & \\
\hline 02.11 .2006 & 3,9 & $-1,9$ & 276,9 & 271,1 & 234,9888 & 5,0112 & \\
\hline 03.11.2006 & 7,1 & -1 & 280,1 & 272 & 233,0016 & 6,9984 & \\
\hline 04.11 .2006 & 9,2 & 3,6 & 282,2 & 276,6 & 235,1616 & 4,8384 & \\
\hline 05.11.2006 & 10,5 & 8,1 & 283,5 & 281,1 & 237,9264 & 2,0736 & \\
\hline 06.11 .2006 & 11,5 & 9,4 & 284,5 & 282,4 & 238,1856 & 1,8144 & \\
\hline 07.11.2006 & 10,2 & 4,7 & 283,2 & 277,7 & 235,248 & 4,752 & \\
\hline 08.11.2006 & 11,2 & 3,6 & 284,2 & 276,6 & 233,4336 & 6,5664 & \\
\hline 09.11.2006 & 10,9 & 5,8 & 283,9 & 278,8 & 235,5936 & 4,4064 & \\
\hline 10.11.2006 & 8,7 & 3,4 & 281,7 & 276,4 & 235,4208 & 4,5792 & \\
\hline 11.11.2006 & 9,2 & 3,4 & 282,2 & 276,4 & 234,9888 & 5,0112 & \\
\hline 12.11.2006 & 7,4 & 3,6 & 280,4 & 276,6 & 236,7168 & 3,2832 & \\
\hline 13.11.2006 & 11,9 & 5,1 & 284,9 & 278,1 & 234,1248 & 5,8752 & \\
\hline 14.11.2006 & 13,6 & 7,4 & 286,6 & 280,4 & 234,6432 & 5,3568 & \\
\hline 15.11.2006 & 14,6 & 7,6 & 287,6 & 280,6 & 233,952 & 6,048 & \\
\hline 16.11.2006 & 16,9 & 8 & 289,9 & 281 & 232,3104 & 7,6896 & \\
\hline 17.11.2006 & 15,1 & 8,3 & 288,1 & 281,3 & 234,1248 & 5,8752 & \\
\hline 18.11.2006 & 12,6 & 3,3 & 285,6 & 276,3 & 231,9648 & 8,0352 & \\
\hline 19.11.2006 & 10 & 2,7 & 283 & 275,7 & 233,6928 & 6,3072 & \\
\hline 20.11.2006 & 7,4 & 2,2 & 280,4 & 275,2 & 235,5072 & 4,4928 & \\
\hline 21.11.2006 & 9,6 & 3,3 & 282,6 & 276,3 & 234,5568 & 5,4432 & \\
\hline 22.11.2006 & 6,2 & 0,8 & 279,2 & 273,8 & 235,3344 & 4,6656 & \\
\hline \multirow[t]{2}{*}{ 23.11.2006 } & 12,8 & 4,6 & 285,8 & 277,6 & 232,9152 & 7,0848 & \\
\hline & & & & & & & 816,307 \\
\hline
\end{tabular}


6.3.2 Berechnungen des Volumenstroms für die unter realitätsnahen Bedingungen (im medizinischen Bereich) gelagerten Testeinheiten und die Kontrollgruppe 2 (2008)

6.3.2.1 Volumenstrom durch wetterabhängige Luftdruckänderungen in Bad Rothenfelde, Oldenburg und bei der Kontrollgruppe 2 (2008) (Boyle- Mariotte- Gesetz)

Bad Rothenfelde (Münster - Osnabrück)

Lagerungszeitraum: 31.03.08 - 10.06.08

(1. Rückführungsetappe) - 30.09.08 (2. Rückführungsetappe) - 08.12 .08 (3. Rückführungsetappe)

\begin{tabular}{|c|c|c|c|c|c|c|}
\hline Datum & $\begin{array}{c}\text { Luftdruck } \\
\text { (p1) }\end{array}$ & $\begin{array}{c}\text { Luftdruck } \\
\text { des Folgetages } \\
\text { (p2) }\end{array}$ & $\mathrm{V} 2=(\mathrm{p} 1 * \mathrm{~V} 1) / \mathrm{p} 2$ & $\begin{array}{l}\text { Luftvolumen- } \\
\text { strom }\end{array}$ & $\begin{array}{c}\begin{array}{c}\text { Luftvolumen- } \\
\text { einstrom } \\
\text { (= positive Werte) }\end{array} \\
\end{array}$ & $\begin{array}{c}\text { Summe } \\
\Delta V\end{array}$ \\
\hline & (hPa) & (hPa) & $(\mathrm{ml})$ & $(\mathrm{ml})$ & $(\mathrm{ml})$ & (ml) \\
\hline 31.03.2008 & 1009,4 & 1013,1 & 239,123482 & 0,876517619 & 0,876517619 & \\
\hline 01.04.2008 & 1013,1 & 1011,9 & 240,284613 & $-0,284613104$ & & \\
\hline 02.04.2008 & 1011,9 & 1017,9 & 238,585323 & 1,414677277 & 1,414677277 & \\
\hline 03.04.2008 & 1017,9 & 1019,1 & 239,717398 & 0,282602296 & 0,282602296 & \\
\hline 04.04 .2008 & 1019,1 & 1002,7 & 243,925401 & $-3,925401416$ & & \\
\hline 05.04.2008 & 1002,7 & 995,4 & 241,760096 & $-1,760096444$ & & \\
\hline 06.04.2008 & 995,4 & 995,9 & 239,879506 & 0,120494026 & 0,120494026 & \\
\hline 07.04.2008 & 995,9 & 999,1 & 239,231308 & 0,768691823 & 0,768691823 & \\
\hline |08.04.2008 & 999,1 & 993,8 & 241,279936 & $-1,279935601$ & & \\
\hline 09.04.2008 & 993,8 & 994 & 239,95171 & 0,048289738 & 0,048289738 & \\
\hline 10.04.2008 & 994 & 994 & 240 & 0 & & \\
\hline 11.04.2008 & 994 & 1001,2 & 238,274071 & 1,725928885 & 1,725928885 & \\
\hline 12.04.2008 & 1001,2 & 1005,1 & 239,068749 & 0,931250622 & 0,931250622 & \\
\hline 13.04.2008 & 1005,1 & 1006,5 & 239,66617 & 0,333830104 & 0,333830104 & \\
\hline 14.04.2008 & 1006,5 & 1013,9 & 238,248348 & 1,751652037 & 1,751652037 & \\
\hline 15.04.2008 & 1013,9 & 1014,3 & 239,905353 & 0,094646554 & 0,094646554 & \\
\hline 16.04.2008 & 1014,3 & 1004,4 & 242,365591 & $-2,365591398$ & & \\
\hline 17.04.2008 & 1004,4 & 997,2 & 241,732852 & $-1,732851986$ & & \\
\hline 18.04.2008 & 997,2 & 996 & 240,289157 & $-0,289156627$ & & \\
\hline 19.04.2008 & 996 & 1001 & 238,801199 & 1,198801199 & 1,198801199 & \\
\hline 20.04.2008 & 1001 & 999,6 & 240,336134 & $-0,336134454$ & & \\
\hline 21.04.2008 & 999,6 & 1003,9 & 238,972009 & 1,027990836 & 1,027990836 & \\
\hline 22.04.2008 & 1003,9 & 1011,8 & 238,126112 & 1,87388812 & 1,87388812 & \\
\hline 23.04.2008 & 1011,8 & 1015,4 & 239,149104 & 0,850896199 & 0,850896199 & \\
\hline 24.04.2008 & 1015,4 & 1019,8 & 238,964503 & 1,035497156 & 1,035497156 & \\
\hline 25.04.2008 & 1019,8 & 1021,5 & 239,600587 & 0,399412628 & 0,399412628 & \\
\hline 26.04.2008 & 1021,5 & 1013,4 & 241,918295 & $-1,918294849$ & & \\
\hline 27.04.2008 & 1013,4 & 1001,1 & 242,948756 & $-2,948756368$ & & \\
\hline 28.04.2008 & 1001,1 & 997,6 & 240,842021 & $-0,84202085$ & & \\
\hline 29.04.2008 & 997,6 & 994,5 & 240,748115 & $-0,74811463$ & & \\
\hline |30.04.2008| & 994,5 & 1004,9 & 237,516171 & 2,483829237 & 2,483829237 & \\
\hline
\end{tabular}




\begin{tabular}{|c|c|c|c|c|c|}
\hline 01.05 .2008 & 1004,9 & 1015,4 & 237,518219 & 2,481780579 & 2,481780579 \\
\hline 02.05.2008 & 1015,4 & 1020,9 & 238,707023 & 1,292976785 & 1,292976785 \\
\hline 03.05.2008 & 1020,9 & 1020 & 240,211765 & $-0,211764706$ & \\
\hline 04.05.2008 & 1020 & 1021,5 & 239,647577 & 0,352422907 & 0,352422907 \\
\hline 05.05.2008 & 1021,5 & 1020,4 & 240,258722 & $-0,25872207$ & \\
\hline 06.05.2008 & 1020,4 & 1016,5 & 240,920807 & $-0,92080669$ & \\
\hline 07.05.2008 & 1016,5 & 1013 & 240,82922 & $-0,829220138$ & \\
\hline 08.05.2008 & 1013 & 1011,1 & 240,450994 & $-0,450993967$ & \\
\hline 09.05.2008 & 1011,1 & 1013 & 239,549852 & 0,450148075 & 0,450148075 \\
\hline 10.05.2008 & 1013 & 1014,2 & 239,716032 & 0,283967659 & 0,283967659 \\
\hline 11.05.2008 & 1014,2 & 1012,5 & 240,402963 & $-0,402962963$ & \\
\hline 12.05 .2008 & 1012,5 & 1010,7 & 240,427427 & $-0,427426536$ & \\
\hline 13.05.2008 & 1010,7 & 1007,9 & 240,666733 & $-0,666732811$ & \\
\hline 14.05.2008 & 1007,9 & 1002,9 & 241,19653 & $-1,196530063$ & \\
\hline 15.05 .2008 & 1002,9 & 1000,9 & 240,479568 & $-0,479568388$ & \\
\hline 16.05 .2008 & 1000,9 & 1000,4 & 240,119952 & $-0,119952019$ & \\
\hline 17.05.2008 & 1000,4 & 1005,2 & 238,853959 & 1,146040589 & 1,146040589 \\
\hline 18.05.2008 & 1005,2 & 1009,1 & 239,072441 & 0,927559211 & 0,927559211 \\
\hline 19.05.2008 & 1009,1 & 1012,1 & 239,288608 & 0,711392155 & 0,711392155 \\
\hline 20.05.2008 & 1012,1 & 1011,8 & 240,07116 & $-0,071160308$ & \\
\hline 21.05.2008 & 1011,8 & 1010,7 & 240,261205 & $-0,261205105$ & \\
\hline 22.05.2008 & 1010,7 & 1009,2 & 240,356718 & $-0,356718193$ & \\
\hline 23.05.2008 & 1009,2 & 1007,1 & 240,500447 & $-0,500446828$ & \\
\hline 24.05 .2008 & 1007,1 & 1007,9 & 239,809505 & 0,190495089 & 0,190495089 \\
\hline 25.05.2008 & 1007,9 & 1006,5 & 240,33383 & $-0,333830104$ & \\
\hline 26.05 .2008 & 1006,5 & 1007,3 & 239,809391 & 0,190608558 & 0,190608558 \\
\hline 27.05.2008 & 1007,3 & 1005,2 & 240,501393 & $-0,501392758$ & \\
\hline 28.05 .2008 & 1005,2 & 1004,9 & 240,071649 & $-0,07164892$ & \\
\hline 29.05.2008 & 1004,9 & 1004 & 240,215139 & $-0,215139442$ & \\
\hline 30.05 .2008 & 1004 & 1007,8 & 239,095059 & 0,904941457 & 0,904941457 \\
\hline 31.05 .2008 & 1007,8 & 1008,9 & 239,738329 & 0,261671127 & 0,261671127 \\
\hline 01.06.2008 & 1008,9 & 1005,8 & 240,73971 & $-0,739709684$ & \\
\hline 02.06.2008 & 1005,8 & 1005 & 240,191045 & $-0,191044776$ & \\
\hline 03.06.2008 & 1005 & 1008,7 & 239,119659 & 0,880341033 & 0,880341033 \\
\hline 04.06 .2008 & 1008,7 & 1008,5 & 240,047595 & $-0,047595439$ & \\
\hline 05.06.2008 & 1008,5 & 1007,4 & 240,262061 & $-0,26206075$ & \\
\hline 06.06 .2008 & 1007,4 & 1008,8 & 239,666931 & 0,333068993 & 0,333068993 \\
\hline 07.06.2008 & 1008,8 & 1013,8 & 238,816335 & 1,183665417 & 1,183665417 \\
\hline 08.06.2008 & 1013,8 & 1016 & 239,480315 & 0,519685039 & 0,519685039 \\
\hline 09.06.2008 & 1016 & 1013,8 & 240,520813 & $-0,520812784$ & \\
\hline 10.06 .2008 & 1013,8 & 1012 & 240,426877 & $-0,42687747$ & \\
\hline 11.06.2008 & 1012 & 1007,3 & 241,119825 & $-1,119825275$ & \\
\hline 12.06.2008 & 1007,3 & 1007,6 & 239,928543 & 0,071456927 & 0,071456927 \\
\hline 13.06.2008 & 1007,6 & 1007,8 & 239,952372 & 0,047628498 & 0,047628498 \\
\hline 14.06 .2008 & 1007,8 & 1003,7 & 240,980373 & $-0,980372621$ & \\
\hline 15.06.2008 & 1003,7 & 1004,4 & 239,832736 & 0,167264038 & 0,167264038 \\
\hline 16.06.2008 & 1004,4 & 1007,4 & 239,285289 & 0,714711138 & 0,714711138 \\
\hline 17.06.2008 & 1007,4 & 1008,4 & 239,761999 & 0,238000793 & 0,238000793 \\
\hline 18.06.2008 & 1008,4 & 1005,4 & 240,716133 & $-0,716132882$ & \\
\hline 19.06.2008 & 1005,4 & 1011,3 & 238,599822 & 1,400177989 & 1,400177989 \\
\hline 20.06.2008 & 1011,3 & 1013,4 & 239,502664 & 0,497335702 & 0,497335702 \\
\hline
\end{tabular}




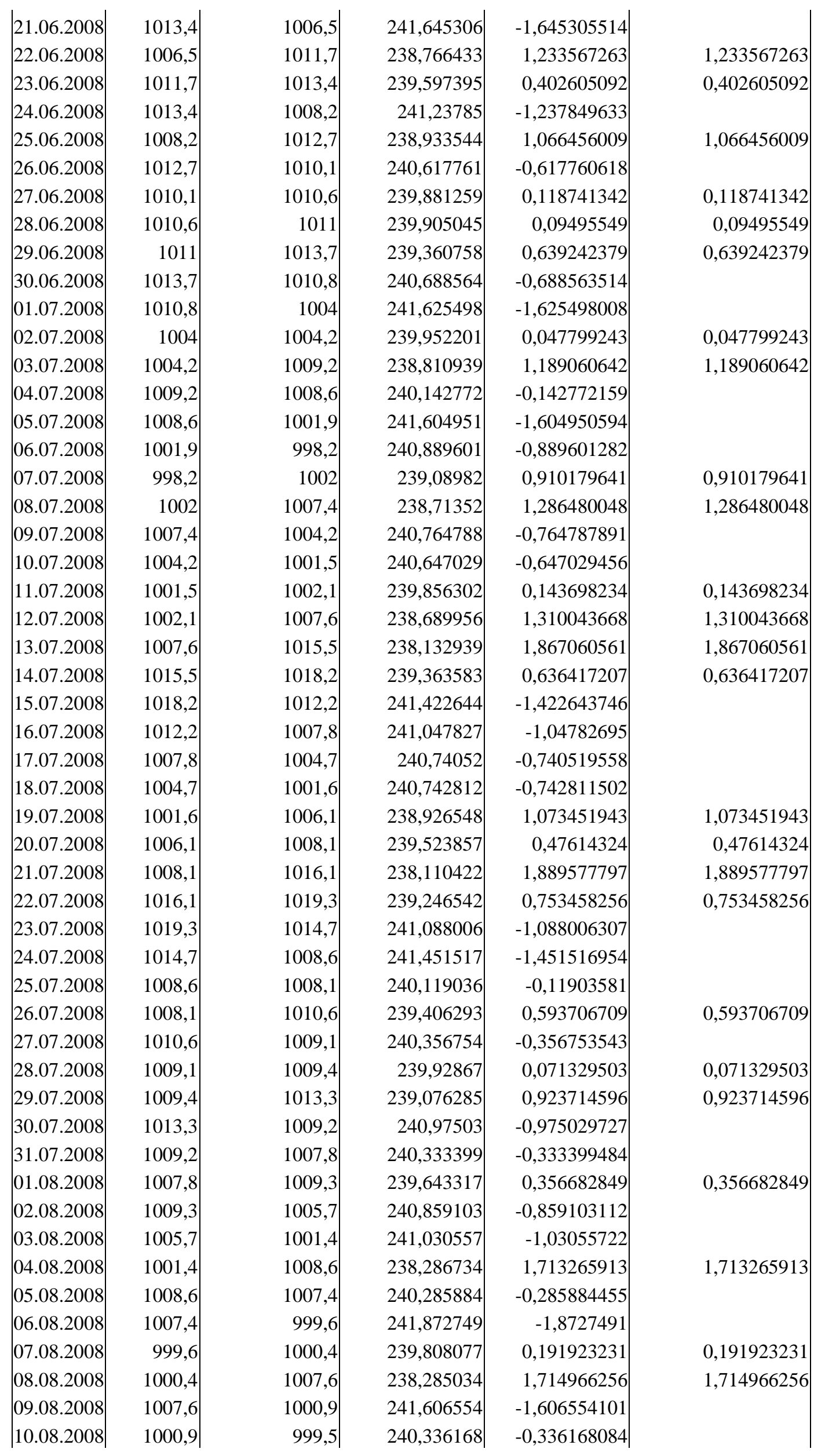




\begin{tabular}{|c|c|c|c|}
\hline 11.08.2008 & 999,5 & 991,3 & 241,985272 \\
\hline 12.08.2008 & 991,3 & 999,1 & 238,126314 \\
\hline 13.08.2008 & 999,1 & 1007,6 & 237,975387 \\
\hline 14.08.2008 & 1007,6 & 1008,3 & 239,833383 \\
\hline 15.08.2008 & 1008,3 & 1007,3 & 240,238261 \\
\hline 16.08.2008 & 1007,3 & 1004 & 240,788845 \\
\hline 17.08.2008 & 1004 & 1003,9 & 240,023907 \\
\hline 18.08.2008 & 1003,9 & 1002,1 & 240,431095 \\
\hline 19.08.2008 & 1002,1 & 1005,4 & 239,212254 \\
\hline 20.08.2008 & 1005,4 & 1009,9 & 238,930587 \\
\hline 21.08.2008 & 1009,9 & 1006,7 & 240,762889 \\
\hline 22.08.2008 & 1006,7 & 1004,9 & 240,429894 \\
\hline 23.08.2008 & 1004,9 & 1007,1 & 239,475722 \\
\hline 24.08.2008 & 1007,1 & 1009,6 & 239,405705 \\
\hline 25.08.2008 & 1009,6 & 1015,1 & 238,699636 \\
\hline 26.08.2008 & 1015,1 & 1015 & 240,023645 \\
\hline 27.08.2008 & 1015 & 1013,1 & 240,450104 \\
\hline 28.08.2008 & 1013,1 & 1012,9 & 240,047389 \\
\hline 29.08.2008 & 1012,9 & 1013,8 & 239,78694 \\
\hline 30.08.2008 & 1013,8 & 1007,5 & 241,500744 \\
\hline 31.08.2008 & 1007,5 & 1008,1 & 239,857157 \\
\hline 01.09.2008 & 1008,1 & 1002,7 & 241,29251 \\
\hline 02.09.2008 & 1002,7 & 999,3 & 240,816572 \\
\hline 03.09.2008 & 999,3 & 999,4 & 239,975986 \\
\hline 04.09.2008 & 999,4 & 997,9 & 240,360758 \\
\hline 05.09.2008 & 997,9 & 997,2 & 240,168472 \\
\hline 06.09.2008 & 997,2 & 1002 & 238,850299 \\
\hline 07.09.2008 & 1002 & 1008,6 & 238,429506 \\
\hline 08.09.2008 & 1008,6 & 1010,1 & 239,6436 \\
\hline 09.09.2008 & 1010,1 & 1008,8 & 240,309278 \\
\hline 10.09.2008 & 1008,8 & 1006,4 & 240,572337 \\
\hline 11.09.2008 & 1006,4 & 1004,8 & 240,382166 \\
\hline 12.09.2008 & 1004,8 & 1012,9 & 238,080758 \\
\hline 13.09.2008 & 1012,9 & 1018,5 & 238,680412 \\
\hline 14.09.2008 & 1018,5 & 1019 & 239,882237 \\
\hline 15.09 .2008 & 1019 & 1017,6 & 240,330189 \\
\hline 16.09 .2008 & 1017,6 & 1017,5 & 240,023587 \\
\hline 17.09.2008 & 1017,5 & 1018,7 & 239,717287 \\
\hline 18.09.2008 & 1018,7 & 1020,5 & 239,576678 \\
\hline 19.09.2008 & 1020,5 & 1024,8 & 238,992974 \\
\hline 20.09.2008 & 1024,8 & 1019,8 & 241,176701 \\
\hline 21.09.2008 & 1019,8 & 1015,2 & 241,08747 \\
\hline 22.09.2008 & 1015,2 & 1012,4 & 240,663769 \\
\hline 23.09.2008 & 1012,4 & 1017,8 & 238,726665 \\
\hline 24.09 .2008 & 1017,8 & 1025,2 & 238,267655 \\
\hline 25.09.2008 & 1025,2 & 1029,2 & 239,067237 \\
\hline 26.09.2008 & 1029,2 & 1024,8 & 241,030445 \\
\hline 27.09.2008 & 1024,8 & 1017,3 & 241,76939 \\
\hline 28.09.2008 & 1017,3 & 1009,2 & 241,926278 \\
\hline 29.09 .2008 & 1009,2 & 997,3 & 242,863732 \\
\hline 30.09 .2008 & 997,3 & 988,9 & 242,038629 \\
\hline
\end{tabular}

\begin{tabular}{|c|c|}
\hline \multicolumn{2}{|l|}{$-1,985271865$} \\
\hline 1,873686318 & 1,873686318 \\
\hline 2,024612942 & 2,024612942 \\
\hline 0,166617078 & 0,166617078 \\
\hline$-0,238260697$ & \\
\hline$-0,788844622$ & \\
\hline$-0,023906764$ & \\
\hline$-0,431094701$ & \\
\hline 0,787746171 & 0,787746171 \\
\hline 1,069412813 & 1,069412813 \\
\hline$-0,762888646$ & \\
\hline$-0,429893522$ & \\
\hline 0,524277629 & 0,524277629 \\
\hline 0,59429477 & 0,59429477 \\
\hline 1,300364496 & 1,300364496 \\
\hline$-0,02364532$ & \\
\hline$-0,450103642$ & \\
\hline$-0,047388686$ & \\
\hline 0,213059775 & 0,213059775 \\
\hline$-1,500744417$ & \\
\hline 0,142842972 & 0,142842972 \\
\hline$-1,292510222$ & \\
\hline$-0,8165716$ & \\
\hline 0,024014409 & 0,024014409 \\
\hline$-0,360757591$ & \\
\hline$-0,168471721$ & \\
\hline 1,149700599 & 1,149700599 \\
\hline 1,570493754 & 1,570493754 \\
\hline 0,356400356 & 0,356400356 \\
\hline$-0,309278351$ & \\
\hline$-0,572337043$ & \\
\hline$-0,382165605$ & \\
\hline 1,919241781 & 1,919241781 \\
\hline 1,319587629 & 1,319587629 \\
\hline 0,117762512 & 0,117762512 \\
\hline$-0,330188679$ & \\
\hline$-0,023587224$ & \\
\hline 0,282713262 & 0,282713262 \\
\hline 0,423321901 & 0,423321901 \\
\hline 1,007025761 & 1,007025761 \\
\hline$-1,176701314$ & \\
\hline$-1,087470449$ & \\
\hline$-0,663769261$ & \\
\hline 1,273334643 & 1,273334643 \\
\hline 1,732344908 & 1,732344908 \\
\hline 0,932763311 & 0,932763311 \\
\hline$-1,030444965$ & \\
\hline$-1,769389561$ & \\
\hline$-1,92627824$ & \\
\hline$-2,863732077$ & \\
\hline$-2,038628779$ & \\
\hline
\end{tabular}

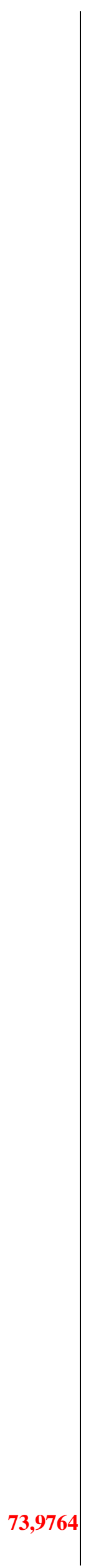




\begin{tabular}{|c|c|c|c|}
\hline 01.10 .2008 & 988,9 & 993,9 & 238,792635 \\
\hline 02.10.2008 & 993,9 & 999,8 & 238,583717 \\
\hline 03.10.2008 & 999,8 & 1006,6 & 238,378701 \\
\hline 04.10 .2008 & 1006,6 & 997,1 & 242,286631 \\
\hline 05.10 .2008 & 997,1 & 1005,7 & 237,947698 \\
\hline 06.10 .2008 & 1005,7 & 1008 & 239,452381 \\
\hline 07.10 .2008 & 1008 & 1010,2 & 239,477331 \\
\hline 08.10.2008 & 1010,2 & 1024 & 236,765625 \\
\hline 09.10.2008 & 1024 & 1026,1 & 239,50882 \\
\hline 10.10.2008 & 1026,1 & 1022 & 240,962818 \\
\hline 11.10 .2008 & 1022 & 1019,8 & 240,517749 \\
\hline 12.10 .2008 & 1019,8 & 1012,5 & 241,73037 \\
\hline 13.10.2008 & 1012,5 & 1013 & 239,88154 \\
\hline 14.10.2008 & 1013 & 1007,4 & 241,334127 \\
\hline 15.10 .2008 & 1007,4 & 1003,6 & 240,908729 \\
\hline 16.10 .2008 & 1003,6 & 1011 & 238,243323 \\
\hline 17.10.2008 & 1011 & 1012,8 & 239,57346 \\
\hline 18.10.2008 & 1012,8 & 1013,8 & 239,763267 \\
\hline 19.10.2008 & 1013,8 & 1006,5 & 241,740686 \\
\hline 20.10.2008 & 1006,5 & 1003,5 & 240,717489 \\
\hline 21.10.2008 & 1003,5 & 1015,3 & 237,210677 \\
\hline 22.10.2008 & 1015,3 & 1020,2 & 238,847285 \\
\hline 23.10.2008 & 1020,2 & 1017,7 & 240,589565 \\
\hline 24.10 .2008 & 1017,7 & 1023,5 & 238,639961 \\
\hline 25.10.2008 & 1023,5 & 1010,4 & 243,111639 \\
\hline 26.10.2008 & 1010,4 & 1000,9 & 242,27795 \\
\hline 27.10 .2008 & 1000,9 & 1002,4 & 239,640862 \\
\hline 28.10.2008 & 1002,4 & 1001,9 & 240,119772 \\
\hline 29.10 .2008 & 1001,9 & 993,6 & 242,004831 \\
\hline 30.10 .2008 & 993,6 & 1000,2 & 238,416317 \\
\hline 31.10 .2008 & 1000,2 & 1003 & 239,33001 \\
\hline 01.11.2008 & 1003 & 1009,8 & 238,383838 \\
\hline 02.11.2008 & 1009,8 & 1008,8 & 240,237906 \\
\hline 03.11 .2008 & 1008,8 & 1013,2 & 238,957758 \\
\hline 04.11 .2008 & 1013,2 & 1008,9 & 241,022896 \\
\hline 05.11 .2008 & 1008,9 & 1009,5 & 239,857355 \\
\hline 06.11 .2008 & 1009,5 & 1007,7 & 240,428699 \\
\hline 07.11 .2008 & 1007,7 & 1011,8 & 239,027476 \\
\hline 08.11 .2008 & 1011,8 & 1008,4 & 240,809203 \\
\hline 09.11 .2008 & 1008,4 & 1004,1 & 241,027786 \\
\hline 10.11.2008 & 1004,1 & 1000,7 & 240,815429 \\
\hline 11.11.2008 & 1000,7 & 1008,7 & 238,09656 \\
\hline 12.11.2008 & 1008,7 & 1017,2 & 237,994495 \\
\hline 13.11.2008 & 1017,2 & 1017,7 & 239,882087 \\
\hline 14.11.2008 & 1017,7 & 1018,1 & 239,905707 \\
\hline 15.11.2008 & 1018,1 & 1019,4 & 239,693938 \\
\hline 16.11 .2008 & 1019,4 & 1022,7 & 239,225579 \\
\hline 17.11.2008 & 1022,7 & 1007 & 243,741807 \\
\hline 18.11.2008 & 1007 & 1008,6 & 239,619274 \\
\hline 19.11.2008 & 1008,6 & 1001,3 & 241,749725 \\
\hline |20.11.2008| & 1001,3 & 994,5 & 241,641026 \\
\hline
\end{tabular}

1,207364926
1,416283257
1,621299424
$-2,286631231$
2,052301879
0,547619048
0,522668778
3,234375

0,491180197

$-0,962818004$

$-0,517748578$

$-1,73037037$

0,11846002

$-1,334127457$

$-0,908728577$

1,756676558

0,426540284

0,236733083

$-1,740685544$

$-0,717488789$

2,789323353

1,152715154

$-0,589564705$

1,360039082

$-3,111638955$

$-2,277949845$

0,359138069

$-0,119772432$

$-2,004830918$

1,583683263

0,66999003

1,616161616

$-0,237906423$

1,0422424

$-1,022896224$

0,142644874

$-0,428699018$

0,972524214

$-0,809202697$

$-1,027786077$

$-0,8154292$

1,903440071

2,005505309

0,117912941

0,094293291

0,30606239

0,774420651

$-3,741807349$

0,380725758

$-1,749725357$

$-1,641025641$

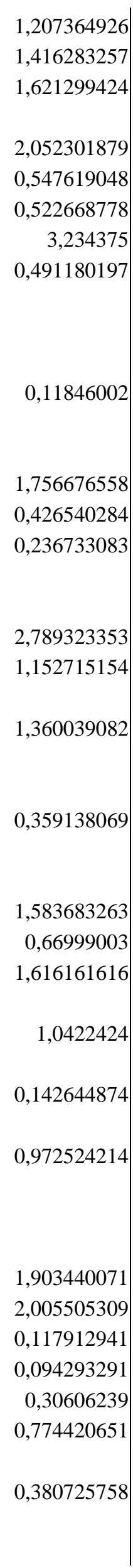




\begin{tabular}{|r|r|r|r|r|r|r|}
21.11 .2008 & 994,5 & 997,2 & 239,350181 & 0,649819495 & 0,649819495 \\
22.11 .2008 & 997,2 & 993,1 & 240,990837 & $-0,990836774$ & \\
23.11 .2008 & 993,1 & 987,4 & 241,385457 & $-1,385456755$ & \\
24.11 .2008 & 987,4 & 1005 & 235,797015 & 4,202985075 & 4,202985075 \\
25.11 .2008 & 1005 & 1016,4 & 237,308146 & 2,691853601 & 2,691853601 \\
26.11 .2008 & 1016,4 & 1010,5 & 241,401286 & $-1,401286492$ & \\
27.11 .2008 & 1010,5 & 998 & 243,006012 & $-3,006012024$ & \\
28.11 .2008 & 998 & 989,7 & 242,012731 & $-2,012731131$ & \\
29.11 .2008 & 989,7 & 989,3 & 240,097038 & $-0,09703831$ & \\
30.11 .2008 & 989,3 & 992,3 & 239,274413 & 0,72558702 & 0,72558702 \\
01.12 .2008 & 992,3 & 996,8 & 238,916533 & 1,083467095 & 1,083467095 \\
02.12 .2008 & 996,8 & 991,3 & 241,331585 & $-1,331584788$ & \\
03.12 .2008 & 991,3 & 986,1 & 241,265592 & $-1,265591725$ & \\
04.12 .2008 & 986,1 & 982,1 & 240,977497 & $-0,9774972$ & \\
05.12 .2008 & 982,1 & 999 & 235,93994 & 4,06006006 & 4,06006006 \\
06.12 .2008 & 999 & 1017,1 & 235,729034 & 4,270966473 & 4,270966473 & \\
07.12 .2008 & 1017,1 & 1017,3 & 239,952816 & 0,047183722 & 0,047183722 & \\
$\mathbf{0 8 . 1 2 . 2 0 0 8}$ & 1017,3 & & & & 122,6106305 & $\mathbf{1 2 2 , 6 1 1}$ \\
\hline & & & & & \\
\end{tabular}

Oldenburg (Bremen)

Lagerungszeitraum: 12.07.08 - 22.09.08

(1. Rückführungsetappe) - 08.12.08 (2. Rückführungsetappe) - 30.03.09 (3. Rückführungsetappe)

\begin{tabular}{|c|c|c|c|c|c|c|}
\hline Datum & $\begin{array}{l}\text { Luftdruck } \\
\text { (p1) }\end{array}$ & \begin{tabular}{|c|} 
Luftdruck \\
des Folgetages \\
(p2)
\end{tabular} & $V 2=(p 1 * V 1) / p 2$ & \begin{tabular}{|c|} 
Luftvolumen- \\
strom
\end{tabular} & $\begin{array}{c}\text { Lufteinstrom- } \\
\text { volumen } \\
\text { (= positive Werte) }\end{array}$ & $\begin{array}{c}\text { Summe } \\
\Delta V\end{array}$ \\
\hline & (hPa) & (hPa) & (ml) & (ml) & (ml) & $(\mathrm{ml})$ \\
\hline 12.07.2008 & 1006,3 & 1011,9 & 238,6718055 & 1,328194486 & \multirow{7}{*}{$\begin{array}{r}1,328194486 \\
1,71899529 \\
0,634112927\end{array}$} & \\
\hline 13.07.2008 & 1011,9 & 1019,2 & 238,2810047 & 1,71899529 & & \\
\hline 14.07 .2008 & 1019,2 & 1021,9 & 239,3658871 & 0,634112927 & & \\
\hline 15.07.2008 & 1021,9 & 1016 & 241,3937008 & $-1,393700787$ & & \\
\hline 16.07.2008 & 1016 & 1011,9 & 240,9724281 & $-0,972428106$ & & \\
\hline 17.07.2008 & 1011,9 & 1009 & 240,6897919 & $-0,689791873$ & & \\
\hline 18.07.2008 & 1009 & 1005,7 & 240,7875112 & $-0,787511186$ & & \\
\hline 19.07.2008 & 1005,7 & 1009,5 & 239,0965825 & 0,903417533 & \multirow{7}{*}{$\begin{array}{r}0,903417533 \\
0,118811881 \\
2,329640161 \\
1,0076157\end{array}$} & \\
\hline 20.07.2008 & 1009,5 & 1010 & 239,8811881 & 0,118811881 & & \\
\hline 21.07.2008 & 1010 & 1019,9 & 237,6703598 & 2,329640161 & & \\
\hline 22.07.2008 & 1019,9 & 1024,2 & 238,9923843 & 1,0076157 & & \\
\hline 23.07.2008 & 1024,2 & 1021,2 & 240,7050529 & $-0,705052879$ & & \\
\hline 24.07.2008 & 1021,2 & 1015,3 & 241,3946617 & $-1,394661676$ & & \\
\hline 25.07.2008 & 1015,3 & 1014,4 & 240,2129338 & $-0,212933754$ & & \\
\hline 26.07.2008 & 1014,4 & 1016,5 & 239,504181 & 0,495818987 & \multirow[t]{2}{*}{0,495818987} & \\
\hline 27.07.2008 & 1016,5 & 1015,9 & 240,1417462 & $-0,141746235$ & & \\
\hline
\end{tabular}




\begin{tabular}{|c|c|c|c|c|c|}
\hline 28.07.2008 & 1015,9 & 1015 & 240,2128079 & $-0,212807882$ & \multirow[b]{2}{*}{0,918637747} \\
\hline 29.07.2008 & 1015 & 1018,9 & 239,0813623 & 0,918637747 & \\
\hline 30.07.2008 & 1018,9 & 1015,8 & 240,7324276 & $-0,732427643$ & \multirow{4}{*}{0,307692308} \\
\hline 31.07.2008 & 1015,8 & 1012,7 & 240,7346697 & $-0,734669695$ & \\
\hline 01.08.2008 & 1012,7 & 1014 & 239,6923077 & 0,307692308 & \\
\hline 02.08.2008 & 1014 & 1009,8 & 240,9982175 & $-0,998217469$ & \\
\hline 03.08.2008 & 1009,8 & 1004 & 241,3864542 & $-1,386454183$ & \multirow{3}{*}{2,085308057} \\
\hline 04.08.2008 & 1004 & 1012,8 & 237,9146919 & 2,085308057 & \\
\hline 05.08.2008 & 1012,8 & 1012,6 & 240,0474027 & $-0,047402726$ & \\
\hline 06.08.2008 & 1012,6 & 1003,9 & 242,0798884 & $-2,079888435$ & \multirow{6}{*}{1,803797468} \\
\hline 07.08.2008 & 1003,9 & 1003,6 & 240,0717417 & $-0,07174173$ & \\
\hline 08.08.2008 & 1003,6 & 1011,2 & 238,1962025 & 1,803797468 & \\
\hline 09.08.2008 & 1011,2 & 1004,3 & 241,6489097 & $-1,648909688$ & \\
\hline 10.08.2008 & 1004,3 & 1003,6 & 240,1673974 & $-0,167397369$ & \\
\hline 11.08.2008 & 1003,6 & 996,1 & 241,8070475 & $-1,807047485$ & \\
\hline 12.08.2008 & 996,1 & 1002,4 & 238,4916201 & 1,508379888 & \multirow{3}{*}{$\begin{array}{l}1,508379888 \\
2,088607595 \\
0,473746546\end{array}$} \\
\hline 13.08.2008 & 1002,4 & 1011,2 & 237,9113924 & 2,088607595 & \\
\hline 14.08.2008 & 1011,2 & 1013,2 & 239,5262535 & 0,473746546 & \\
\hline 15.08.2008 & 1013,2 & 1012,3 & 240,2133755 & $-0,213375482$ & \multirow{7}{*}{$\begin{array}{l}0,594707107 \\
1,183548673\end{array}$} \\
\hline 16.08.2008 & 1012,3 & 1009,5 & 240,6656761 & $-0,665676077$ & \\
\hline 17.08.2008 & 1009,5 & 1008,8 & 240,1665345 & $-0,166534496$ & \\
\hline 18.08.2008 & 1008,8 & 1006,4 & 240,572337 & $-0,572337043$ & \\
\hline 19.08.2008 & 1006,4 & 1008,9 & 239,4052929 & 0,594707107 & \\
\hline 20.08.2008 & 1008,9 & 1013,9 & 238,8164513 & 1,183548673 & \\
\hline 21.08.2008 & 1013,9 & 1011,3 & 240,6170276 & $-0,617027588$ & \\
\hline 22.08.2008 & 1011,3 & 1008,1 & 240,7618292 & $-0,761829184$ & \multirow{5}{*}{$\begin{array}{l}0,735759494 \\
0,686322848 \\
1,200941915\end{array}$} \\
\hline 23.08.2008 & 1008,1 & 1011,2 & 239,2642405 & 0,735759494 & \\
\hline 24.08.2008 & 1011,2 & 1014,1 & 239,3136772 & 0,686322848 & \\
\hline 25.08.2008 & 1014,1 & 1019,2 & 238,7990581 & 1,200941915 & \\
\hline 26.08.2008 & 1019,2 & 1018,6 & 240,1413705 & $-0,141370509$ & \\
\hline 27.08.2008 & 1018,6 & 1016,5 & 240,495819 & $-0,495818987$ & \multirow{3}{*}{$\begin{array}{l}0,141578999 \\
0,705813156\end{array}$} \\
\hline 28.08.2008 & 1016,5 & 1017,1 & 239,858421 & 0,141578999 & \\
\hline 29.08.2008 & 1017,1 & 1020,1 & 239,2941868 & 0,705813156 & \\
\hline 30.08.2008 & 1020,1 & 1013,8 & 241,4914184 & $-1,491418426$ & \multirow{7}{*}{$\begin{array}{r}0,11962915 \\
0,023923445\end{array}$} \\
\hline 31.08.2008 & 1013,8 & 1011,7 & 240,4981714 & $-0,498171395$ & \\
\hline 01.09.2008 & 1011,7 & 1006,9 & 241,1441057 & $-1,144105671$ & \\
\hline 02.09.2008 & 1006,9 & 1002,6 & 241,0293238 & $-1,029323758$ & \\
\hline 03.09.2008 & 1002,6 & 1003,1 & 239,8803709 & 0,11962915 & \\
\hline 04.09.2008 & 1003,1 & 1003,2 & 239,9760766 & 0,023923445 & \\
\hline 05.09.2008 & 1003,2 & 1001,9 & 240,3114083 & $-0,311408324$ & \\
\hline 06.09.2008 & 1001,9 & 1006,8 & 238,8319428 & 1,168057211 & \multirow{3}{*}{$\begin{array}{l}1,168057211 \\
1,303961276 \\
0,756277696\end{array}$} \\
\hline 07.09.2008 & 1006,8 & 1012,3 & 238,6960387 & 1,303961276 & \\
\hline 08.09.2008 & 1012,3 & 1015,5 & 239,2437223 & 0,756277696 & \\
\hline 09.09.2008 & 1015,5 & 1013,4 & 240,4973357 & $-0,497335702$ & \multirow{8}{*}{$\begin{array}{l}0,165811291 \\
1,833496572 \\
1,006534673\end{array}$} \\
\hline 10.09.2008 & 1013,4 & 1012,5 & 240,2133333 & $-0,213333333$ & \\
\hline 11.09.2008 & 1012,5 & 1013,2 & 239,8341887 & 0,165811291 & \\
\hline 12.09 .2008 & 1013,2 & 1021 & 238,1665034 & 1,833496572 & \\
\hline 13.09.2008 & 1021 & 1025,3 & 238,9934653 & 1,006534673 & \\
\hline 14.09.2008 & 1025,3 & 1024,7 & 240,1405289 & $-0,140528935$ & \\
\hline 15.09.2008 & 1024,7 & 1023,3 & 240,3283495 & $-0,328349458$ & \\
\hline 16.09.2008 & 1023,3 & 1023 & 240,0703812 & $-0,070381232$ & \\
\hline
\end{tabular}




\begin{tabular}{|c|c|c|c|c|c|c|}
\hline 17.09 .2008 & 1023 & 1024,6 & 239,6252196 & 0,374780402 & 0,374780402 & \multirow{51}{*}{31,0766} \\
\hline 18.09.2008 & 1024,6 & 1026,7 & 239,5091068 & 0,490893153 & \multirow{2}{*}{$\begin{array}{l}0,490893153 \\
0,861801242\end{array}$} & \\
\hline 19.09.2008 & 1026,7 & 1030,4 & 239,1381988 & 0,861801242 & & \\
\hline 20.09.2008 & 1030,4 & 1025 & 241,2643902 & $-1,264390244$ & \multirow{11}{*}{$\begin{array}{r}1,546421947 \\
1,721430648 \\
0,85763956\end{array}$} & \\
\hline 21.09.2008 & 1025 & 1020,6 & 241,0346855 & $-1,034685479$ & & \\
\hline 22.09 .2008 & 1020,6 & 1017,7 & 240,6838951 & $-0,683895057$ & & \\
\hline 23.09.2008 & 1017,7 & 1024,3 & 238,4535781 & 1,546421947 & & \\
\hline 24.09.2008 & 1024,3 & 1031,7 & 238,2785694 & 1,721430648 & & \\
\hline 25.09.2008 & 1031,7 & 1035,4 & 239,1423604 & 0,85763956 & & \\
\hline 26.09.2008 & 1035,4 & 1030,3 & 241,1880035 & $-1,188003494$ & & \\
\hline 27.09.2008 & 1030,3 & 1021,9 & 241,9727958 & $-1,972795773$ & & \\
\hline 28.09.2008 & 1021,9 & 1013,9 & 241,8936779 & $-1,893677878$ & & \\
\hline 29.09 .2008 & 1013,9 & 1000,2 & 243,2873425 & $-3,287342531$ & & \\
\hline 30.09 .2008 & 1000,2 & 991,2 & 242,1791768 & $-2,179176755$ & & \\
\hline 01.10 .2008 & 991,2 & 996,5 & 238,7235324 & 1,276467637 & 1,276467637 & \\
\hline 02.10 .2008 & 996,5 & 1003,7 & 238,27837 & 1,721629969 & 1,721629969 & \\
\hline 03.10 .2008 & 1003,7 & 1010,2 & 238,4557513 & 1,544248664 & 1,544248664 & \\
\hline 04.10 .2008 & 1010,2 & 1001,1 & 242,1816002 & $-2,18160024$ & & \\
\hline 05.10.2008 & 1001,1 & 1011,5 & 237,5323777 & 2,467622343 & 2,467622343 & \\
\hline 06.10 .2008 & 1011,5 & 1014,1 & 239,3846761 & 0,615323933 & 0,615323933 & \\
\hline 07.10 .2008 & 1014,1 & 1015,1 & 239,7635701 & 0,236429908 & 0,236429908 & \\
\hline 08.10 .2008 & 1015,1 & 1028,6 & 236,8500875 & 3,149912502 & 3,149912502 & \\
\hline 09.10 .2008 & 1028,6 & 1030,7 & 239,5110119 & 0,488988066 & 0,488988066 & \\
\hline 10.10.2008 & 1030,7 & 1026,5 & 240,9819776 & $-0,981977594$ & & \\
\hline 11.10.2008 & 1026,5 & 1024,6 & 240,4450517 & $-0,445051728$ & & \\
\hline 12.10.2008 & 1024,6 & 1017,1 & 241,7697375 & $-1,769737489$ & & \\
\hline 13.10.2008 & 1017,1 & 1017,3 & 239,9528163 & 0,047183722 & 0,047183722 & \\
\hline 14.10.2008 & 1017,3 & 1011,7 & 241,3284571 & $-1,328457052$ & & \\
\hline 15.10.2008 & 1011,7 & 1006,8 & 241,1680572 & $-1,168057211$ & & \\
\hline 16.10.2008 & 1006,8 & 1014,3 & 238,2253771 & 1,774622893 & 1,774622893 & \\
\hline 17.10.2008 & 1014,3 & 1017,2 & 239,3157688 & 0,684231223 & 0,684231223 & \\
\hline 18.10.2008 & 1017,2 & 1018 & 239,8113949 & 0,188605108 & 0,188605108 & \\
\hline 19.10.2008 & 1018 & 1011,3 & 241,5900326 & $-1,590032631$ & & \\
\hline 20.10.2008 & 1011,3 & 1007,9 & 240,8096041 & $-0,809604127$ & & \\
\hline 21.10 .2008 & 1007,9 & 1020,2 & 237,1064497 & 2,893550284 & 2,893550284 & \\
\hline 22.10.2008 & 1020,2 & 1025,2 & 238,8294967 & 1,170503316 & 1,170503316 & \\
\hline 23.10.2008 & 1025,2 & 1022,6 & 240,6102093 & $-0,61020927$ & & \\
\hline 24.10 .2008 & 1022,6 & 1028,7 & 238,5768446 & 1,423155439 & 1,423155439 & \\
\hline 25.10.2008 & 1028,7 & 1014,3 & 243,407276 & $-3,407275954$ & & \\
\hline 26.10 .2008 & 1014,3 & 1005,2 & 242,1727019 & $-2,17270195$ & & \\
\hline 27.10.2008 & 1005,2 & 1007,1 & 239,5472148 & 0,452785225 & 0,452785225 & \\
\hline 28.10.2008 & 1007,1 & 1007,8 & 239,8333003 & 0,166699742 & 0,166699742 & \\
\hline 29.10.2008 & 1007,8 & 999,8 & 241,9203841 & $-1,920384077$ & & \\
\hline 30.10 .2008 & 999,8 & 1007,5 & 238,1657568 & 1,834243176 & 1,834243176 & \\
\hline 31.10 .2008 & 1007,5 & 1011,1 & 239,1454851 & 0,854514885 & 0,854514885 & \\
\hline 01.11 .2008 & 1011,1 & 1017,2 & 238,560755 & 1,439244986 & 1,439244986 & \\
\hline 02.11.2008 & 1017,2 & 1016,5 & 240,165273 & $-0,165272996$ & & \\
\hline 03.11 .2008 & 1016,5 & 1019,8 & 239,2233771 & 0,776622867 & 0,776622867 & \\
\hline 04.11 .2008 & 1019,8 & 1016,8 & 240,7081039 & $-0,708103855$ & & \\
\hline 05.11 .2008 & 1016,8 & 1016 & 240,1889764 & $-0,188976378$ & & \\
\hline 06.11 .2008 & 1016 & 1013,7 & 240,5445398 & $-0,544539805$ & & \\
\hline
\end{tabular}




\begin{tabular}{|r|r|r|}
\hline 07.11 .2008 & 1013,7 & 1017,7 \\
08.11 .2008 & 1017,7 & 1013,6 \\
09.11 .2008 & 1013,6 & 1008,5 \\
10.11 .2008 & 1008,5 & 1004,5 \\
11.11 .2008 & 1004,5 & 1013 \\
12.11 .2008 & 1013 & 1022,2 \\
13.11 .2008 & 1022,2 & 1021,3 \\
14.11 .2008 & 1021,3 & 1021,4 \\
15.11 .2008 & 1021,4 & 1022,7 \\
16.11 .2008 & 1022,7 & 1027,6 \\
17.11 .2008 & 1027,6 & 1010,9 \\
18.11 .2008 & 1010,9 & 1010,9 \\
19.11 .2008 & 1010,9 & 1003 \\
20.11 .2008 & 1003 & 997,3 \\
21.11 .2008 & 997,3 & 1000,3 \\
22.11 .2008 & 1000,3 & 998,1 \\
23.11 .2008 & 998,1 & 993,6 \\
24.11 .2008 & 993,6 & 1009,7 \\
25.11 .2008 & 1009,7 & 1019,8 \\
26.11 .2008 & 1019,8 & 1014,6 \\
27.11 .2008 & 1014,6 & 1003,9 \\
28.11 .2008 & 1003,9 & 995,4 \\
29.11 .2008 & 995,4 & 996,7 \\
30.11 .2008 & 996,7 & 998,1 \\
01.12 .2008 & 998,1 & 1000,8 \\
02.12 .2008 & 1000,8 & 996,2 \\
03.12 .2008 & 996,2 & 992 \\
04.12 .2008 & 992 & 988 \\
05.12 .2008 & 988 & 1004,1 \\
06.12 .2008 & 1004,1 & 1021,5 \\
07.12 .2008 & 1021,5 & 1021,5 \\
$\mathbf{0 8 . 1 2 . 2 0 0 8}$ & 1021,5 & 1012,1 \\
09.12 .2008 & 1012,1 & 1011,6 \\
10.12 .2008 & 1011,6 & 1012,8 \\
11.12 .2008 & 1012,8 & 1016,3 \\
12.12 .2008 & 1016,3 & 1009 \\
13.12 .2008 & 1009 & 1006 \\
14.12 .2008 & 1006 & 1015,8 \\
15.12 .2008 & 1015,8 & 1016 \\
16.12 .2008 & 1016 & 1011,2 \\
17.12 .2008 & 1011,2 & 1016,5 \\
18.12 .2008 & 1016,5 & 1017 \\
19.12 .2008 & 1017 \\
20.12 .2008 & 1016,3 & 1016,3 \\
21.12 .2008 & 1024,4 & 1024,4 \\
22.12 .2008 & 1025,9 & 1025,9 \\
23.12 .2008 & 1032,8 & 1032,8 \\
24.12 .2008 & 1029,2 & 1029,2 \\
25.12 .2008 & 1036,7 & 1036,7 \\
26.12 .2008 & 1043,2 & 1043,2 \\
27.12 .2008 & 1039,7 & 1039,7 \\
& & 1035,3 \\
\hline
\end{tabular}

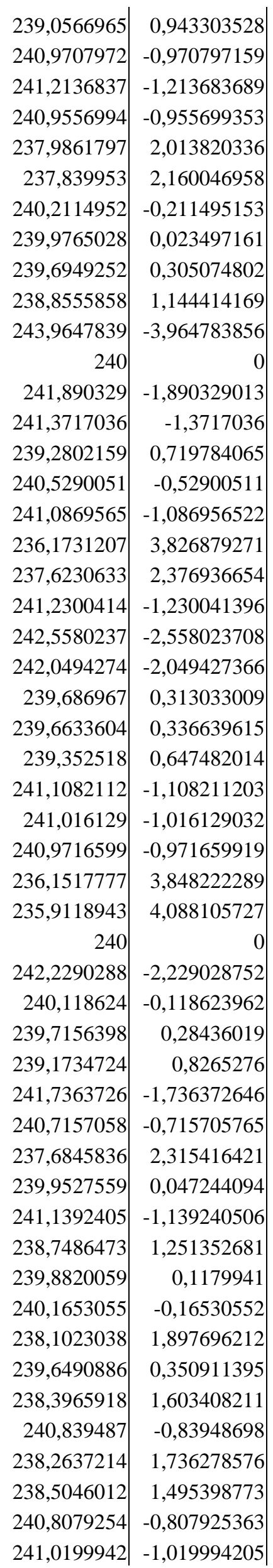




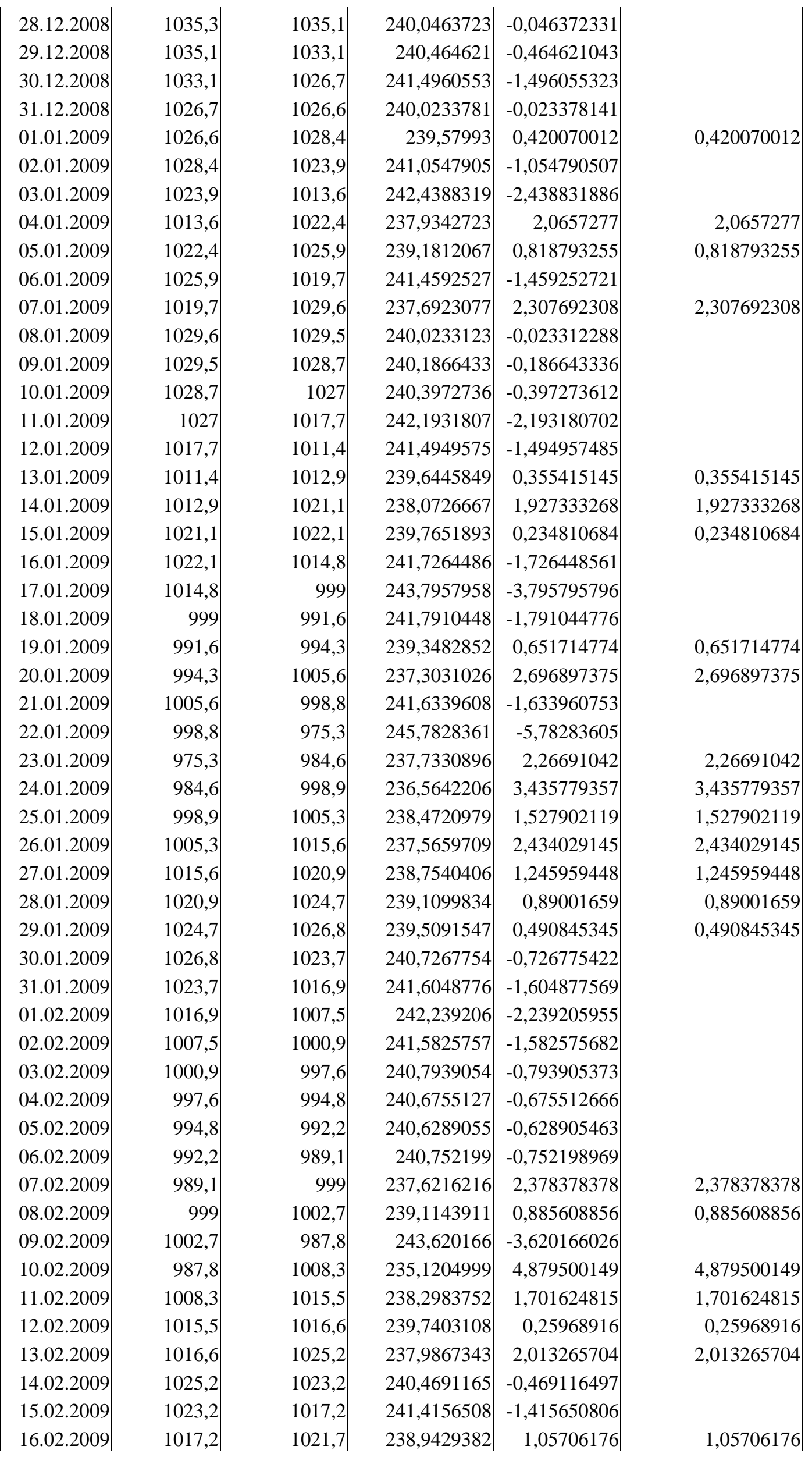




\begin{tabular}{|c|c|c|c|c|c|c|}
\hline 17.02.2009 & 1021,7 & 1028 & 238,5291829 & 1,470817121 & \multirow{2}{*}{\multicolumn{2}{|c|}{1,470817121}} \\
\hline 18.02.2009 & 1028 & 1023,9 & 240,9610314 & $-0,961031351$ & & \\
\hline 19.02.2009 & 1023,9 & 1024,1 & 239,9531296 & 0,046870423 & \multirow{4}{*}{\multicolumn{2}{|c|}{$\begin{array}{l}0,046870423 \\
0,863981319\end{array}$}} \\
\hline 20.02.2009 & 1024,1 & 1027,8 & 239,1360187 & 0,863981319 & & \\
\hline 21.02.2009 & 1027,8 & 1016,9 & 242,5725243 & $-2,572524339$ & & \\
\hline 22.02.2009 & 1016,9 & 1014,1 & 240,6626565 & $-0,662656543$ & & \\
\hline 23.02.2009 & 1014,1 & 1024,5 & 237,5636896 & 2,436310395 & \multirow{6}{*}{\multicolumn{2}{|c|}{2,436310395}} \\
\hline 24.02.2009 & 1024,5 & 1024,4 & 240,0234283 & $-0,023428348$ & & \\
\hline 25.02.2009 & 1024,4 & 1014 & 242,4615385 & $-2,461538462$ & & \\
\hline 26.02.2009 & 1014 & 1013,8 & 240,0473466 & $-0,047346617$ & & \\
\hline 27.02.2009 & 1013,8 & 1013,6 & 240,047356 & $-0,047355959$ & & \\
\hline 28.02.2009 & 1013,6 & 1009,8 & 240,9031491 & $-0,903149138$ & & \\
\hline 01.03.2009 & 1009,8 & 1011 & 239,7151335 & 0,284866469 & \multirow{4}{*}{\multicolumn{2}{|c|}{0,284866469}} \\
\hline 02.03.2009 & 1011 & 1010,9 & 240,0237412 & $-0,023741221$ & & \\
\hline 03.03.2009 & 1010,9 & 993,4 & 244,2279042 & $-4,227904168$ & & \\
\hline 04.03.2009 & 993,4 & 988,7 & 241,1408921 & $-1,140892081$ & & \\
\hline 05.03.2009 & 988,7 & 996 & 238,2409639 & 1,759036145 & & 1,759036145 \\
\hline 06.03.2009 & 996 & 1008,1 & 237,1193334 & 2,880666601 & \multirow{2}{*}{\multicolumn{2}{|c|}{2,880666601}} \\
\hline 07.03.2009 & 1008,1 & 1001,2 & 241,6540152 & $-1,654015182$ & & \\
\hline 08.03.2009 & 1001,2 & 1002,9 & 239,5931798 & 0,406820221 & & 0,406820221 \\
\hline 09.03.2009 & 1002,9 & 1006,5 & 239,1415797 & 0,858420268 & & 0,858420268 \\
\hline 10.03.2009 & 1006,5 & 1017,3 & 237,452079 & 2,547920967 & \multirow{2}{*}{\multicolumn{2}{|c|}{2,547920967}} \\
\hline 11.03.2009 & 1017,3 & 1016,2 & 240,2597914 & $-0,25979138$ & & \\
\hline 12.03.2009 & 1016,2 & 1018 & 239,5756385 & 0,424361493 & \multirow{2}{*}{\multicolumn{2}{|c|}{0,424361493}} \\
\hline 13.03.2009 & 1018 & 1014,9 & 240,7330772 & $-0,73307715$ & & \\
\hline 14.03.2009 & 1014,9 & 1021,5 & 238,4493392 & 1,550660793 & \multirow{2}{*}{\multicolumn{2}{|c|}{$\begin{array}{l}1,550660793 \\
2,326708677\end{array}$}} \\
\hline 15.03.2009 & 1021,5 & 1031,5 & 237,6732913 & 2,326708677 & & \\
\hline 16.03.2009 & 1031,5 & 1032,4 & 239,7907788 & 0,209221232 & \multirow{3}{*}{\multicolumn{2}{|c|}{0,209221232}} \\
\hline 17.03.2009 & 1032,4 & 1031,6 & 240,1861187 & $-0,186118651$ & & \\
\hline 18.03.2009 & 1031,6 & 1028,3 & 240,7702032 & $-0,770203248$ & & \\
\hline 19.03.2009 & 1028,3 & 1032,5 & 239,0237288 & 0,976271186 & \multirow{4}{*}{\multicolumn{2}{|c|}{ 0,976271186 }} \\
\hline 20.03.2009 & 1032,5 & 1027 & 241,285297 & $-1,285296981$ & & \\
\hline 21.03.2009 & 1027 & 1020,3 & 241,5760071 & $-1,576007057$ & & \\
\hline 22.03.2009 & 1020,3 & 1003,5 & 244,0179372 & $-4,01793722$ & & \\
\hline 23.03.2009 & 1003,5 & 1004,4 & 239,7849462 & 0,215053763 & \multirow{2}{*}{\multicolumn{2}{|c|}{0,215053763}} \\
\hline 24.03.2009 & 1004,4 & 998,6 & 241,3939515 & $-1,393951532$ & & \\
\hline 25.03.2009 & 998,6 & 998,9 & 239,9279207 & 0,072079287 & \multirow{3}{*}{\multicolumn{2}{|c|}{0,072079287}} \\
\hline 26.03.2009 & 998,9 & 995,3 & 240,86808 & $-0,868079976$ & & \\
\hline 27.03.2009 & 995,3 & 995 & 240,0723618 & $-0,072361809$ & & \\
\hline 28.03.2009 & 995 & 1008 & 236,9047619 & 3,095238095 & \multirow{3}{*}{\multicolumn{2}{|c|}{$\begin{array}{l}3,095238095 \\
2,870025485\end{array}$}} \\
\hline 29.03.2009 & 1008 & 1020,2 & 237,1299745 & 2,870025485 & & \\
\hline 30.03.2009 & 1020,2 & & & & & \\
\hline
\end{tabular}


Göttingen (Kontrollprobe 2 (2008))

Lagerungszeitraum: 31.03.08 - 31.05.08

\begin{tabular}{|c|c|c|c|c|c|c|}
\hline Datum & $\begin{array}{l}\text { Luftdruck } \\
\text { (p1) }\end{array}$ & $\begin{array}{c}\text { Luftdruck } \\
\text { des Folgetages } \\
\text { (p2) }\end{array}$ & $V 2=(p 1 * V 1) / p 2$ & $\begin{array}{c}\text { Luftvolumen- } \\
\text { strom }\end{array}$ & $\begin{array}{c}\begin{array}{c}\text { Luftvolumen- } \\
\text { einstrom }\end{array} \\
\text { (= positive Werte) }\end{array}$ & $\begin{array}{c}\text { Summe } \\
\Delta V\end{array}$ \\
\hline & (hPa) & (ml) & (ml) & $(\mathrm{ml})$ & (ml) & $(\mathrm{ml})$ \\
\hline 31.03.2008 & 1014 & 1014 & 240 & 0 & & \\
\hline 01.04.2008 & 1014 & 1016 & 239,5275591 & 0,47244094 & 0,472440945 & \\
\hline 02.04.2008 & 1016 & 1021 & 238,8246817 & 1,17531832 & 1,175318315 & \\
\hline 03.04.2008 & 1021 & 1027 & 238,5978578 & 1,40214216 & 1,402142162 & \\
\hline 04.04.2008 & 1027 & 1008 & 244,5238095 & $-4,52380952$ & & \\
\hline 05.04.2008 & 1008 & 999 & 242,1621622 & $-2,16216216$ & & \\
\hline 06.04.2008 & 999 & 997 & 240,4814443 & $-0,48144433$ & & \\
\hline 07.04 .2008 & 997 & 1006 & 237,8528827 & 2,1471173 & 2,147117296 & \\
\hline 08.04.2008 & 1006 & 997 & 242,1664995 & $-2,1664995$ & & \\
\hline 09.04.2008 & 997 & 999 & 239,5195195 & 0,48048048 & 0,48048048 & \\
\hline 10.04.2008 & 999 & 997 & 240,4814443 & $-0,48144433$ & & \\
\hline 11.04 .2008 & 997 & 1006 & 237,8528827 & 2,1471173 & 2,147117296 & \\
\hline 12.04 .2008 & 1006 & 1011 & 238,8130564 & 1,18694362 & 1,18694362 & \\
\hline 13.04.2008 & 1011 & 1009 & 240,4757185 & $-0,47571853$ & & \\
\hline 14.04.2008 & 1009 & 1017 & 238,1120944 & 1,8879056 & 1,887905605 & \\
\hline 15.04.2008 & 1017 & 1021 & 239,0597453 & 0,94025465 & 0,940254652 & \\
\hline 16.04.2008 & 1021 & 1008 & 243,0952381 & $-3,0952381$ & & \\
\hline 17.04 .2008 & 1008 & 998 & 242,4048096 & $-2,40480962$ & & \\
\hline 18.04.2008 & 998 & 1009 & 237,3835481 & 2,61645193 & 2,616451933 & \\
\hline 19.04.2008 & 1009 & 1003 & 241,4356929 & $-1,43569292$ & & \\
\hline 20.04.2008 & 1003 & 1008 & 238,8095238 & 1,19047619 & 1,19047619 & \\
\hline 21.04 .2008 & 1008 & 1016 & 238,1102362 & 1,88976378 & 1,88976378 & \\
\hline 22.04 .2008 & 1016 & 1018 & 239,5284872 & 0,47151277 & 0,47151277 & \\
\hline 23.04.2008 & 1018 & 1021 & 239,294809 & 0,70519099 & 0,705190989 & \\
\hline 24.04.2008 & 1021 & 1024 & 239,296875 & 0,703125 & 0,703125 & \\
\hline 25.04.2008 & 1024 & 1029 & 238,8338192 & 1,16618076 & 1,166180758 & \\
\hline 26.04.2008 & 1029 & 1018 & 242,5933202 & $-2,59332024$ & & \\
\hline 27.04.2008 & 1018 & 1002 & 243,8323353 & $-3,83233533$ & & \\
\hline 28.04.2008 & 1002 & 1003 & 239,7607178 & 0,23928215 & 0,239282154 & \\
\hline 29.04.2008 & 1003 & 998 & 241,2024048 & $-1,20240481$ & & \\
\hline 30.04.2008 & 998 & 1010 & 237,1485149 & 2,85148515 & 2,851485149 & \\
\hline 01.05.2008 & 1010 & 1021 & 237,4142997 & 2,58570029 & 2,585700294 & \\
\hline 02.05 .2008 & 1021 & 1028 & 238,3657588 & 1,63424125 & 1,634241245 & \\
\hline 03.05.2008 & 1028 & 1033 & 238,8383349 & 1,16166505 & 1,161665053 & \\
\hline 04.05 .2008 & 1033 & 1027 & 241,4021422 & $-1,40214216$ & & \\
\hline 05.05.2008 & 1027 & 1023 & 240,9384164 & $-0,93841642$ & & \\
\hline 06.05.2008 & 1023 & 1021 & 240,4701273 & $-0,47012733$ & & \\
\hline 07.05 .2008 & 1021 & 1017 & 240,9439528 & $-0,9439528$ & & \\
\hline 08.05.2008 & 1017 & 1015 & 240,4729064 & $-0,4729064$ & & \\
\hline
\end{tabular}




\begin{tabular}{|r|r|r|r|r|r|r|}
09.05 .2008 & 1015 & 1019 & 239,0578999 & 0,9421001 & 0,942100098 \\
10.05 .2008 & 1019 & 1020 & 239,7647059 & 0,23529412 & 0,235294118 \\
11.05 .2008 & 1020 & 1016 & 240,9448819 & $-0,94488189$ & \\
12.05 .2008 & 1016 & 1014 & 240,4733728 & $-0,47337278$ & \\
13.05 .2008 & 1014 & 1012 & 240,4743083 & $-0,4743083$ & \\
14.05 .2008 & 1012 & 1007 & 241,1916584 & $-1,19165839$ & \\
15.05 .2008 & 1007 & 1005 & 240,4776119 & $-0,47761194$ & \\
16.05 .2008 & 1005 & 1001 & 240,959041 & $-0,95904096$ & \\
17.05 .2008 & 1001 & 1009 & 238,0971259 & 1,90287413 & 1,902874133 \\
18.05 .2008 & 1009 & 1012 & 239,2885375 & 0,71146245 & 0,711462451 \\
19.05 .2008 & 1012 & 1015 & 239,2906404 & 0,70935961 & 0,709359606 \\
20.05 .2008 & 1015 & 1017 & 239,5280236 & 0,4719764 & 0,471976401 \\
21.05 .2008 & 1017 & 1015 & 240,4729064 & $-0,4729064$ & \\
22.05 .2008 & 1015 & 1013 & 240,4738401 & $-0,47384008$ & \\
23.05 .2008 & 1013 & 1011 & 240,4747774 & $-0,47477745$ & \\
24.05 .2008 & 1011 & 1014 & 239,2899408 & 0,71005917 & 0,710059172 \\
25.05 .2008 & 1014 & 1010 & 240,950495 & $-0,95049505$ & \\
26.05 .2008 & 1010 & 1014 & 239,0532544 & 0,94674556 & 0,946745562 \\
27.05 .2008 & 1014 & 1011 & 240,7121662 & $-0,71216617$ & \\
28.05 .2008 & 1011 & 1013 & 239,5261599 & 0,47384008 & 0,473840079 \\
29.05 .2008 & 1013 & 1005 & 241,9104478 & $-1,91044776$ & \\
30.05 .2008 & 1005 & 1012 & 238,3399209 & 1,66007905 & 1,660079051 \\
31.05 .2008 & 1012 & & & \\
\hline
\end{tabular}


6.3.2.2 Volumenstrom durch Temperaturänderungen am Standort Oldenburg (Charles`sches Gesetz)

Oldenburg (Bremen)

Lagerungszeitraum: 12.07.08 - 22.09.08

(1. Rückführungsetappe) - 08.12.08 (2. Rückführungsetappe) - 30.03.09 (3. Rückführungsetappe)

\begin{tabular}{|c|c|c|c|c|c|c|c|}
\hline Datum & $\begin{array}{c}\text { T1 } \\
\text { (max. } \\
\text { Temp.) }\end{array}$ & $\begin{array}{c}\text { T2 } \\
\text { (min. } \\
\text { Temp.) }\end{array}$ & T1 & $\mathbf{T} 2$ & $\begin{array}{c}240 *(1+0,0036 * \\
(\mathrm{T} 2-\mathrm{T} 1)) \\
=\mathrm{V} 2\end{array}$ & $\begin{array}{c}\text { Luftvolumen- } \\
\text { einstrom } \\
\text { (=V1-V2) }\end{array}$ & $\begin{array}{c}\text { Summe } \\
\Delta \mathbf{V}\end{array}$ \\
\hline & $\left({ }^{\circ} \mathrm{C}\right)$ & $\left({ }^{\circ} \mathrm{C}\right)$ & (Kelvin) & (Kelvin) & $(\mathrm{ml})$ & (ml) & (ml) \\
\hline 12.07 .2008 & 19,9 & 11,5 & 292,9 & 284,5 & 232,7424 & 7,2576 & \\
\hline 13.07.2008 & 21,2 & 8,3 & 294,2 & 281,3 & 228,8544 & 11,1456 & \\
\hline $\mid 14.07 .2008$ & 22,3 & 11,4 & 295,3 & 284,4 & 230,5824 & 9,4176 & \\
\hline 15.07 .2008 & 22,3 & 12,5 & 295,3 & 285,5 & 231,5328 & 8,4672 & \\
\hline 16.07.2008 & 20 & 10,7 & 293 & 283,7 & 231,9648 & 8,0352 & \\
\hline 17.07.2008 & 20,5 & 11,6 & 293,5 & 284,6 & 232,3104 & 7,6896 & \\
\hline 18.07.2008 & 17,8 & 13,1 & 290,8 & 286,1 & 235,9392 & 4,0608 & \\
\hline 19.07.2008 & 19,8 & 12,3 & 292,8 & 285,3 & 233,52 & 6,48 & \\
\hline 20.07.2008 & 18,1 & 12 & 291,1 & 285 & 234,7296 & 5,2704 & \\
\hline 21.07.2008 & 15,9 & 10,8 & 288,9 & 283,8 & 235,5936 & 4,4064 & \\
\hline 22.07.2008 & 18,7 & 9 & 291,7 & 282 & 231,6192 & 8,3808 & \\
\hline 23.07.2008 & 21,7 & 8,5 & 294,7 & 281,5 & 228,5952 & 11,4048 & \\
\hline 24.07.2008 & 26 & 11,5 & 299 & 284,5 & 227,472 & 12,528 & \\
\hline 25.07.2008 & 28,3 & 15,8 & 301,3 & 288,8 & 229,2 & 10,8 & \\
\hline 26.07.2008 & 30,7 & 17,4 & 303,7 & 290,4 & 228,5088 & 11,4912 & \\
\hline 27.07.2008 & 31,7 & 17,9 & 304,7 & 290,9 & 228,0768 & 11,9232 & \\
\hline 28.07.2008 & 31,4 & 16,6 & 304,4 & 289,6 & 227,2128 & 12,7872 & \\
\hline 29.07.2008 & 32,5 & 18,1 & 305,5 & 291,1 & 227,5584 & 12,4416 & \\
\hline 30.07 .2008 & 26,6 & 16,3 & 299,6 & 289,3 & 231,1008 & 8,8992 & \\
\hline 31.07.2008 & 30,5 & 16,6 & 303,5 & 289,6 & 227,9904 & 12,0096 & \\
\hline 01.08 .2008 & 27,1 & 16,7 & 300,1 & 289,7 & 231,0144 & 8,9856 & \\
\hline 02.08 .2008 & 25 & 13,3 & 298 & 286,3 & 229,8912 & 10,1088 & \\
\hline 03.08.2008 & 23,6 & 16,5 & 296,6 & 289,5 & 233,8656 & 6,1344 & \\
\hline 04.08 .2008 & 20,4 & 14,5 & 293,4 & 287,5 & 234,9024 & 5,0976 & \\
\hline 05.08 .2008 & 22,2 & 13,4 & 295,2 & 286,4 & 232,3968 & 7,6032 & \\
\hline 06.08 .2008 & 26,6 & 15,5 & 299,6 & 288,5 & 230,4096 & 9,5904 & \\
\hline 07.08.2008 & 30 & 16,5 & 303 & 289,5 & 228,336 & 11,664 & \\
\hline 08.08.2008 & 21,7 & 12,1 & 294,7 & 285,1 & 231,7056 & 8,2944 & \\
\hline 09.08.2008 & 20 & 10,2 & 293 & 283,2 & 231,5328 & 8,4672 & \\
\hline 10.08.2008 & 20,3 & 14,9 & 293,3 & 287,9 & 235,3344 & 4,6656 & \\
\hline 11.08.2008 & 22 & 13,3 & 295 & 286,3 & 232,4832 & 7,5168 & \\
\hline 12.08.2008 & 23,2 & 14,7 & 296,2 & 287,7 & 232,656 & 7,344 & \\
\hline 13.08.2008 & 19,8 & 13,7 & 292,8 & 286,7 & 234,7296 & 5,2704 & \\
\hline 14.08 .2008 & 21 & 9,6 & 294 & 282,6 & 230,1504 & 9,8496 & \\
\hline $\mid$ & 21,7 & 8,8 & 294,7 & 281,8 & 228,8544 & 11,1456 & \\
\hline
\end{tabular}




\begin{tabular}{|c|c|c|c|c|}
\hline 16.08.2008 & 22,5 & 7,7 & 295,5 & 280,7 \\
\hline 17.08.2008 & 24 & 8,3 & 297 & 281,3 \\
\hline 18.08.2008 & 18,7 & 14,7 & 291,7 & 287,7 \\
\hline 19.08.2008 & 22,5 & 14,1 & 295,5 & 287,1 \\
\hline 20.08.2008 & 20,1 & 13,9 & 293,1 & 286,9 \\
\hline 21.08.2008 & 22,3 & 13 & 295,3 & 286 \\
\hline 22.08.2008 & 20,7 & 12,8 & 293,7 & 285,8 \\
\hline 23.08.2008 & 14,5 & 11,8 & 287,5 & 284,8 \\
\hline 24.08.2008 & 19,7 & 9,3 & 292,7 & 282,3 \\
\hline 25.08.2008 & 20,5 & 13,4 & 293,5 & 286,4 \\
\hline 26.08.2008 & 19,5 & 13,4 & 292,5 & 286,4 \\
\hline 27.08.2008 & 18 & 15,5 & 291 & 288,5 \\
\hline 28.08.2008 & 20,5 & 15,8 & 293,5 & 288,8 \\
\hline 29.08.2008 & 21,4 & 12,4 & 294,4 & 285,4 \\
\hline 30.08 .2008 & 22,3 & 10 & 295,3 & 283 \\
\hline 31.08.2008 & 25,5 & 12,3 & 298,5 & 285,3 \\
\hline 01.09.2008 & 21,5 & 11,8 & 294,5 & 284,8 \\
\hline 02.09 .2008 & 20,2 & 10,5 & 293,2 & 283,5 \\
\hline 03.09.2008 & 18,4 & 10,6 & 291,4 & 283,6 \\
\hline 04.09.2008 & 18,9 & 9,7 & 291,9 & 282,7 \\
\hline 05.09.2008 & 21,1 & 12,1 & 294,1 & 285,1 \\
\hline 06.09.2008 & 22,2 & 13,8 & 295,2 & 286,8 \\
\hline 07.09.2008 & 20,7 & 12,4 & 293,7 & 285,4 \\
\hline 08.09.2008 & 19,6 & 11,2 & 292,6 & 284,2 \\
\hline 09.09.2008 & 22,6 & 10,1 & 295,6 & 283,1 \\
\hline 10.09 .2008 & 22,5 & 13,5 & 295,5 & 286,5 \\
\hline 11.09 .2008 & 25,6 & 12,4 & 298,6 & 285,4 \\
\hline 12.09.2008 & 21,7 & 13 & 294,7 & 286 \\
\hline 13.09.2008 & 17 & 7,6 & 290 & 280,6 \\
\hline 14.09.2008 & 16,2 & 8,3 & 289,2 & 281,3 \\
\hline 15.09.2008 & 17,6 & 9,3 & 290,6 & 282,3 \\
\hline 16.09 .2008 & 13,9 & 8,9 & 286,9 & 281,9 \\
\hline 17.09.2008 & 16,8 & 5,7 & 289,8 & 278,7 \\
\hline 18.09.2008 & 16,8 & 5,2 & 289,8 & 278,2 \\
\hline 19.09.2008 & 16 & 3,8 & 289 & 276,8 \\
\hline 20.09.2008 & 18,5 & 1,5 & 291,5 & 274,5 \\
\hline 21.09.2008 & 17,5 & 6,9 & 290,5 & 279,9 \\
\hline 22.09 .2008 & 14,8 & 9 & 287,8 & 282 \\
\hline 23.09.2008 & 13,7 & 11,2 & 286,7 & 284,2 \\
\hline 24.09.2008 & 17,8 & 8,3 & 290,8 & 281,3 \\
\hline 25.09.2008 & 18,6 & 8,5 & 291,6 & 281,5 \\
\hline 26.09 .2008 & 17,4 & 3,9 & 290,4 & 276,9 \\
\hline 27.09.2008 & 18,3 & 2,9 & 291,3 & 275,9 \\
\hline 28.09.2008 & 17,8 & 4,6 & 290,8 & 277,6 \\
\hline 29.09.2008 & 11,7 & 7 & 284,7 & 280 \\
\hline 30.09 .2008 & 12 & 7,8 & 285 & 280,8 \\
\hline 01.10 .2008 & 14,3 & 8,5 & 287,3 & 281,5 \\
\hline 02.10 .2008 & 12,1 & 6,3 & 285,1 & 279,3 \\
\hline 03.10.2008 & 12,3 & 5,7 & 285,3 & 278,7 \\
\hline 04.10 .2008 & 11,7 & 5,4 & 284,7 & 278,4 \\
\hline |05.10.2008 & 11,7 & 8,3 & 284,7 & 281,3 \\
\hline
\end{tabular}

$\mid 227,2128$
226,4352
236,544
232,7424
234,6432
231,9648
233,1744
237,6672
231,0144
233,8656
234,7296
237,84
235,9392
232,224
229,3728
228,5952
231,6192
231,6192
233,2608
232,0512
232,224
232,7424
232,8288
232,7424
229,2
232,224
228,5952
232,4832
231,8784
233,1744
232,8288
235,68
230,4096
229,9776
229,4592
225,312
230,8416
234,9888
237,84
231,792
231,2736
228,336
226,6944
228,5952
235,9392
236,3712
234,9888
234,9888
234,2976
234,5568
237,0624

12,7872

13,5648

3,456

7,2576

5,3568

8,0352

6,8256

2,3328

8,9856

6,1344

5,2704

2,16

4,0608

7,776

10,6272

11,4048

8,3808

8,3808

6,7392

7,9488

7,776

7,2576

7,1712

7,2576

10,8

7,776

11,4048

7,5168

8,1216

6,8256

7,1712

4,32

9,5904

10,0224

10,5408

14,688

9,1584

5,0112

2,16

8,208

8,7264

11,664

13,3056

11,4048

4,0608

3,6288

5,0112

5,0112

5,7024

5,4432

2,9376 


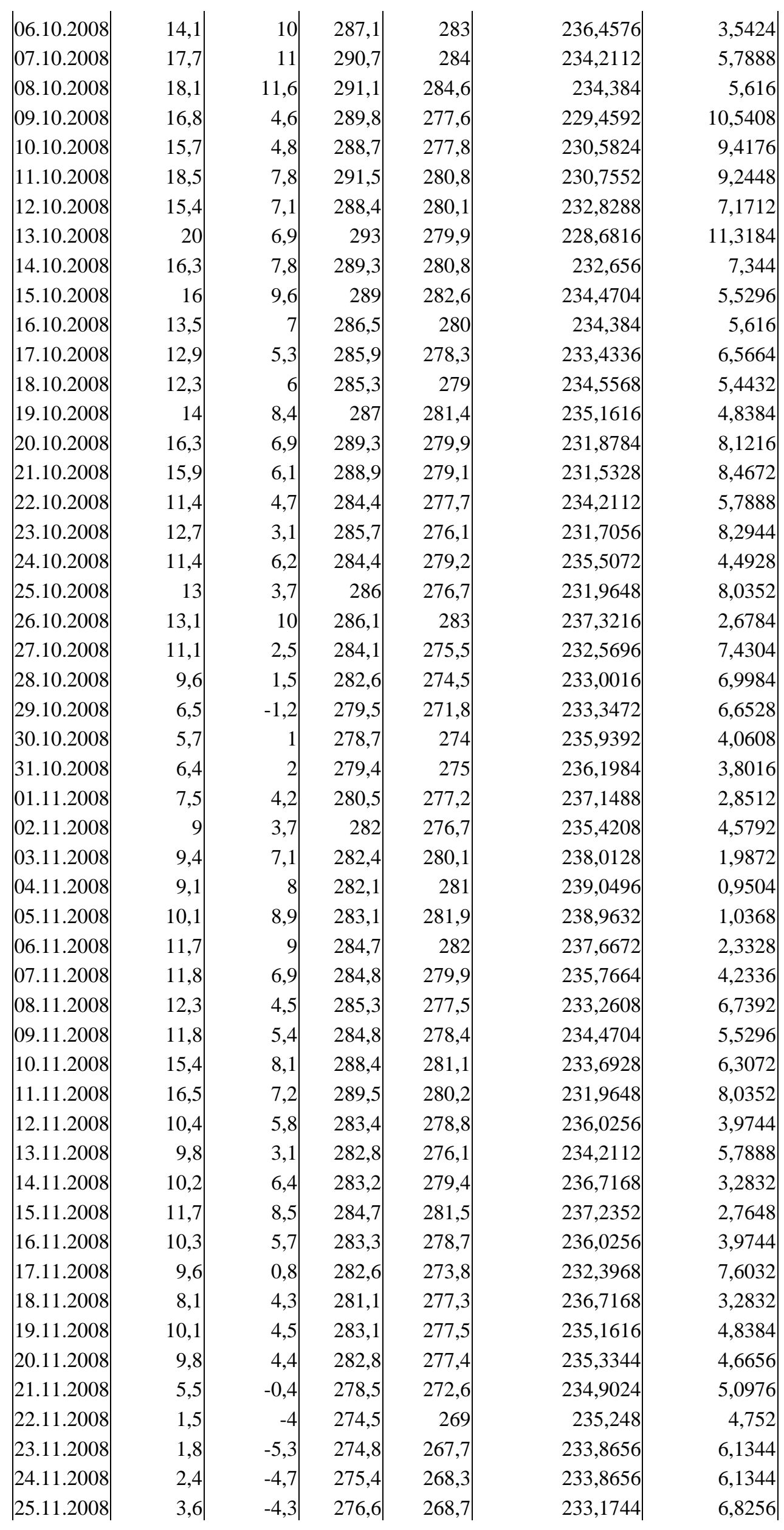




\begin{tabular}{|c|c|c|c|c|c|c|c|}
\hline 26.11.2008 & 6,2 & 0,3 & 279,2 & 273,3 & 234,9024 & 5,0976 & \multirow{51}{*}{1029,8016} \\
\hline 27.11.2008 & 7 & 5,4 & 280 & 278,4 & 238,6176 & 1,3824 & \\
\hline 28.11.2008 & 5,8 & 0,4 & 278,8 & 273,4 & 235,3344 & 4,6656 & \\
\hline 29.11.2008 & 2,9 & 0,1 & 275,9 & 273,1 & 237,5808 & 2,4192 & \\
\hline 30.11 .2008 & 1,7 & $-2,1$ & 274,7 & 270,9 & 236,7168 & 3,2832 & \\
\hline 01.12 .2008 & 3,1 & $-1,7$ & 276,1 & 271,3 & 235,8528 & 4,1472 & \\
\hline 02.12 .2008 & 5,7 & 0,8 & 278,7 & 273,8 & 235,7664 & 4,2336 & \\
\hline 03.12.2008 & 3 & 0,5 & 276 & 273,5 & 237,84 & 2,16 & \\
\hline 04.12 .2008 & 5,3 & 1,3 & 278,3 & 274,3 & 236,544 & 3,456 & \\
\hline 05.12.2008 & 6,7 & 2,8 & 279,7 & 275,8 & 236,6304 & 3,3696 & \\
\hline 06.12.2008 & 6 & 1,9 & 279 & 274,9 & 236,4576 & 3,5424 & \\
\hline 07.12 .2008 & 7,6 & 0,6 & 280,6 & 273,6 & 233,952 & 6,048 & \\
\hline 08.12.2008 & 5,7 & $-0,9$ & 278,7 & 272,1 & 234,2976 & 5,7024 & \\
\hline 09.12 .2008 & 1,4 & $-1,9$ & 274,4 & 271,1 & 237,1488 & 2,8512 & \\
\hline 10.12.2008 & 2,4 & $-1,3$ & 275,4 & 271,7 & 236,8032 & 3,1968 & \\
\hline 11.12.2008 & 2,5 & $-1,8$ & 275,5 & 271,2 & 236,2848 & 3,7152 & \\
\hline 12.12.2008 & 1,6 & -3 & 274,6 & 270 & 236,0256 & 3,9744 & \\
\hline 13.12.2008 & 2,1 & $-1,6$ & 275,1 & 271,4 & 236,8032 & 3,1968 & \\
\hline 14.12 .2008 & 4,4 & 0,3 & 277,4 & 273,3 & 236,4576 & 3,5424 & \\
\hline 15.12 .2008 & 4,2 & 2,6 & 277,2 & 275,6 & 238,6176 & 1,3824 & \\
\hline 16.12 .2008 & 3,7 & 1,9 & 276,7 & 274,9 & 238,4448 & 1,5552 & \\
\hline 17.12.2008 & 3,4 & 1,5 & 276,4 & 274,5 & 238,3584 & 1,6416 & \\
\hline 18.12.2008 & 5,7 & 0,3 & 278,7 & 273,3 & 235,3344 & 4,6656 & \\
\hline 19.12.2008 & 7 & 2,5 & 280 & 275,5 & 236,112 & 3,888 & \\
\hline 20.12.2008 & 9,1 & 3,6 & 282,1 & 276,6 & 235,248 & 4,752 & \\
\hline 21.12.2008 & 9,6 & 0,8 & 282,6 & 273,8 & 232,3968 & 7,6032 & \\
\hline 22.12 .2008 & 9,9 & 5,2 & 282,9 & 278,2 & 235,9392 & 4,0608 & \\
\hline 23.12.2008 & 7,7 & 5,3 & 280,7 & 278,3 & 237,9264 & 2,0736 & \\
\hline 24.12 .2008 & 7,7 & 2,9 & 280,7 & 275,9 & 235,8528 & 4,1472 & \\
\hline 25.12.2008 & 5,7 & $-1,4$ & 278,7 & 271,6 & 233,8656 & 6,1344 & \\
\hline 26.12.2008 & 0,6 & $-3,4$ & 273,6 & 269,6 & 236,544 & 3,456 & \\
\hline 27.12.2008 & 1,2 & $-3,6$ & 274,2 & 269,4 & 235,8528 & 4,1472 & \\
\hline 28.12.2008 & 2,2 & $-4,5$ & 275,2 & 268,5 & 234,2112 & 5,7888 & \\
\hline 29.12.2008 & $-2,2$ & $-5,1$ & 270,8 & 267,9 & 237,4944 & 2,5056 & \\
\hline 30.12.2008 & $-2,5$ & $-8,4$ & 270,5 & 264,6 & 234,9024 & 5,0976 & \\
\hline 31.12 .2008 & $-2,4$ & $-9,4$ & 270,6 & 263,6 & 233,952 & 6,048 & \\
\hline 01.01 .2009 & 1,7 & $-2,8$ & 274,7 & 270,2 & 236,112 & 3,888 & \\
\hline 02.01 .2009 & 1,3 & -8 & 274,3 & 265 & 231,9648 & 8,0352 & \\
\hline 03.01 .2009 & 3 & -8 & 276 & 265 & 230,496 & 9,504 & \\
\hline 04.01 .2009 & 3,9 & 0,4 & 276,9 & 273,4 & 236,976 & 3,024 & \\
\hline 05.01 .2009 & 0,5 & $-8,1$ & 273,5 & 264,9 & 232,5696 & 7,4304 & \\
\hline 06.01 .2009 & $-2,3$ & $-10,8$ & 270,7 & 262,2 & 232,656 & 7,344 & \\
\hline 07.01 .2009 & 0,4 & $-10,4$ & 273,4 & 262,6 & 230,6688 & 9,3312 & \\
\hline 08.01.2009 & $-4,5$ & $-11,1$ & 268,5 & 261,9 & 234,2976 & 5,7024 & \\
\hline 09.01 .2009 & $-0,7$ & $-7,1$ & 272,3 & 265,9 & 234,4704 & 5,5296 & \\
\hline 10.01 .2009 & 1,8 & $-7,3$ & 274,8 & 265,7 & 232,1376 & 7,8624 & \\
\hline 11.01.2009 & 1,8 & -6 & 274,8 & 267 & 233,2608 & 6,7392 & \\
\hline 12.01 .2009 & 7,1 & $-1,8$ & 280,1 & 271,2 & 232,3104 & 7,6896 & \\
\hline 13.01.2009 & 6,4 & 3,4 & 279,4 & 276,4 & 237,408 & 2,592 & \\
\hline 14.01 .2009 & 4,1 & 2,6 & 277,1 & 275,6 & 238,704 & 1,296 & \\
\hline$|15.01 .2009|$ & 3,3 & 1,2 & 276,3 & 274,2 & 238,1856 & 1,8144 & \\
\hline
\end{tabular}




\begin{tabular}{|c|c|c|c|c|c|}
\hline 16.01.2009 & 2,2 & 0 & 275,2 & 273 & 238,0992 \\
\hline 17.01.2009 & 5,2 & 0,2 & 278,2 & 273,2 & 235,68 \\
\hline 18.01.2009 & 5,8 & 2,1 & 278,8 & 275,1 & 236,8032 \\
\hline 19.01.2009 & 7,4 & 1,1 & 280,4 & 274,1 & 234,5568 \\
\hline 20.01.2009 & 6,2 & $-0,4$ & 279,2 & 272,6 & 234,2976 \\
\hline 21.01.2009 & 4,5 & $-2,6$ & 277,5 & 270,4 & 233,8656 \\
\hline 22.01.2009 & 5 & $-1,2$ & 278 & 271,8 & 234,6432 \\
\hline 23.01.2009 & 4,1 & 0,7 & 277,1 & 273,7 & 237,0624 \\
\hline 24.01.2009 & 5,8 & 1,6 & 278,8 & 274,6 & 236,3712 \\
\hline 25.01.2009 & 5,2 & $-0,6$ & 278,2 & 272,4 & 234,9888 \\
\hline 26.01.2009 & 0,5 & $-3,8$ & 273,5 & 269,2 & 236,2848 \\
\hline 27.01.2009 & $-0,5$ & $-2,9$ & 272,5 & 270,1 & 237,9264 \\
\hline 28.01.2009 & $-1,2$ & $-3,5$ & 271,8 & 269,5 & 238,0128 \\
\hline 29.01.2009 & 1,1 & -3 & 274,1 & 270 & 236,4576 \\
\hline 30.01 .2009 & $-0,1$ & $-3,2$ & 272,9 & 269,8 & 237,3216 \\
\hline 31.01.2009 & 2,8 & $-1,7$ & 275,8 & 271,3 & 236,112 \\
\hline 01.02.2009 & 0,8 & $-3,8$ & 273,8 & 269,2 & 236,0256 \\
\hline 02.02.2009 & 2,6 & -1 & 275,6 & 272 & 236,8896 \\
\hline 03.02.2009 & 2,4 & 1 & 275,4 & 274 & 238,7904 \\
\hline 04.02 .2009 & 2,1 & 0,4 & 275,1 & 273,4 & 238,5312 \\
\hline 05.02.2009 & 5,1 & 0,3 & 278,1 & 273,3 & 235,8528 \\
\hline 06.02.2009 & 9,4 & 3 & 282,4 & 276 & 234,4704 \\
\hline 07.02.2009 & 4,1 & $-3,8$ & 277,1 & 269,2 & 233,1744 \\
\hline 08.02.2009 & 5,7 & $-3,1$ & 278,7 & 269,9 & 232,3968 \\
\hline 09.02.2009 & 2,1 & $-2,7$ & 275,1 & 270,3 & 235,8528 \\
\hline 10.02.2009 & 4 & 0,2 & 277 & 273,2 & 236,7168 \\
\hline 11.02.2009 & 4,4 & $-2,7$ & 277,4 & 270,3 & 233,8656 \\
\hline 12.02.2009 & 3,9 & $-3,7$ & 276,9 & 269,3 & 233,4336 \\
\hline 13.02.2009 & 2,8 & $-2,9$ & 275,8 & 270,1 & 235,0752 \\
\hline 14.02 .2009 & 1,1 & $-6,8$ & 274,1 & 266,2 & 233,1744 \\
\hline 15.02.2009 & 2,1 & $-3,9$ & 275,1 & 269,1 & 234,816 \\
\hline 16.02.2009 & 5,6 & 2 & 278,6 & 275 & 236,8896 \\
\hline 17.02.2009 & 4,8 & $-3,5$ & 277,8 & 269,5 & 232,8288 \\
\hline 18.02.2009 & 1,4 & $-6,1$ & 274,4 & 266,9 & 233,52 \\
\hline 19.02.2009 & 2,3 & $-4,1$ & 275,3 & 268,9 & 234,4704 \\
\hline 20.02.2009 & 5,8 & 1,8 & 278,8 & 274,8 & 236,544 \\
\hline 21.02.2009 & 6,5 & 2,1 & 279,5 & 275,1 & 236,1984 \\
\hline 22.02.2009 & 8,3 & 4,6 & 281,3 & 277,6 & 236,8032 \\
\hline 23.02.2009 & 5,8 & 3,1 & 278,8 & 276,1 & 237,6672 \\
\hline 24.02.2009 & 5,6 & 0,5 & 278,6 & 273,5 & 235,5936 \\
\hline 25.02.2009 & 6,4 & 3,1 & 279,4 & 276,1 & 237,1488 \\
\hline 26.02.2009 & 9,1 & 5,1 & 282,1 & 278,1 & 236,544 \\
\hline 27.02.2009 & 7,6 & 2,1 & 280,6 & 275,1 & 235,248 \\
\hline 28.02.2009 & 9,6 & 6,5 & 282,6 & 279,5 & 237,3216 \\
\hline 01.03.2009 & 10,2 & 5,6 & 283,2 & 278,6 & 236,0256 \\
\hline 02.03.2009 & 8 & 4,5 & 281 & 277,5 & 236,976 \\
\hline 03.03.2009 & 9,4 & 0,3 & 282,4 & 273,3 & 232,1376 \\
\hline 04.03.2009 & 10,8 & 2,3 & 283,8 & 275,3 & 232,656 \\
\hline 05.03.2009 & 7,2 & 1 & 280,2 & 274 & 234,6432 \\
\hline 06.03.2009 & 5,8 & 3,7 & 278,8 & 276,7 & 238,1856 \\
\hline 07.03.2009 & 8,1 & 3,2 & 281,1 & 276,2 & 235,7664 \\
\hline
\end{tabular}




\begin{tabular}{|c|c|c|c|c|c|c|c|}
\hline |08.03.2009| & 10 & 1,2 & 283 & 274,2 & 232,3968 & 7,6032 & \\
\hline 09.03.2009 & 6,2 & 1,9 & 279,2 & 274,9 & 236,2848 & 3,7152 & \\
\hline 10.03.2009 & 7 & 2,4 & 280 & 275,4 & 236,0256 & 3,9744 & \\
\hline 11.03.2009 & 9,1 & 0,3 & 282,1 & 273,3 & 232,3968 & 7,6032 & \\
\hline 12.03.2009 & 8,8 & 2 & 281,8 & 275 & 234,1248 & 5,8752 & \\
\hline 13.03.2009| & 11,5 & 1,7 & 284,5 & 274,7 & 231,5328 & 8,4672 & \\
\hline 14.03.2009 & 12,5 & 3,3 & 285,5 & 276,3 & 232,0512 & 7,9488 & \\
\hline 15.03.2009 & 8,2 & 5,1 & 281,2 & 278,1 & 237,3216 & 2,6784 & \\
\hline 16.03.2009 & 8,6 & 5,4 & 281,6 & 278,4 & 237,2352 & 2,7648 & \\
\hline 17.03.2009 & 10,6 & 1,4 & 283,6 & 274,4 & 232,0512 & 7,9488 & \\
\hline 18.03.2009 & 9,8 & $-0,8$ & 282,8 & 272,2 & 230,8416 & 9,1584 & \\
\hline 19.03.2009 & 10,2 & 1 & 283,2 & 274 & 232,0512 & 7,9488 & \\
\hline 20.03.2009 & 8,5 & $-0,7$ & 281,5 & 272,3 & 232,0512 & 7,9488 & \\
\hline 21.03.2009 & 10,7 & $-2,7$ & 283,7 & 270,3 & 228,4224 & 11,5776 & \\
\hline 22.03.2009 & 9,9 & 4,9 & 282,9 & 277,9 & 235,68 & 4,32 & \\
\hline 23.03.2009 & 7,4 & 1,2 & 280,4 & 274,2 & 234,6432 & 5,3568 & \\
\hline 24.03.2009 & 6,6 & $-3,3$ & 279,6 & 269,7 & 231,4464 & 8,5536 & \\
\hline 25.03.2009 & 4,3 & $-1,4$ & 277,3 & 271,6 & 235,0752 & 4,9248 & \\
\hline 26.03.2009| & 7,9 & 1,9 & 280,9 & 274,9 & 234,816 & 5,184 & \\
\hline 27.03.2009 & 9,8 & 4,3 & 282,8 & 277,3 & 235,248 & 4,752 & \\
\hline 28.03.2009 & 9,3 & 2,3 & 282,3 & 275,3 & 233,952 & 6,048 & \\
\hline 29.03.2009 & 9,8 & $-0,6$ & 282,8 & 272,4 & 231,0144 & 8,9856 & \\
\hline 30.03.2009 & 11,2 & $-2,1$ & 284,2 & 270,9 & 228,5088 & 11,4912 & 1581,12 \\
\hline
\end{tabular}




\section{Danksagung}

Mit dem Zeitpunkt der Fertigstellung dieser Dissertationsschrift ist der Moment gekommen, ein Wort des Dankes an die Personen zu richten, die zur Erstellung selbiger beigetragen haben.

Besonderer Dank gilt zunächst Herrn Professor Dr. med. Hartmut Dunkelberg, der mir ermöglichte diese Arbeit im Institut für Hygiene und Umweltmedizin durchzuführen. Seine wissenschaftliche und zielgerichtete Betreuung dieses Projektes habe ich genauso geschätzt wie die kritischen und ideenreichen Fachdiskussionen mit ihm.

Des Weiteren danke ich Herrn Dr. med. Ulrich Schmelz für seine wertvollen Ratschläge, er war stets ein Ansprechpartner mit neuen Ideen und Anregungen für das Projekt.

Für die Unterstützung dieser Arbeit durch Logistik und Bereitstellung entsprechender Lagerungsmöglichkeiten richtet sich mein Dank an Frau C. Abrahamson (Schüchtermann- Klinik Bad Rothenfelde) und an Herrn Dr. med. J. Herrmann ( Institut für Krankenhaushygiene, Klinikum Oldenburg). 


\section{Lebenslauf}

Mein Name ist Katharina Niemeyer, wohnhaft in der Wilhelm- Raabe- Strasse 35 in 37603 Holzminden. Geboren wurde ich am 06. Januar 1985 in Holzminden. Ich wuchs als Tochter von Gerlinde Borgolte (Drogistin) und Dietmar Borgolte (Berufssoldat) gemeinsam mit einem zwei Jahre älteren Bruder auf. Vom August 1991 bis zum Juli 1995 besuchte ich die katholische Grundschule Stahle. Anschließend absolvierte ich im Juni 2004 am König- Wilhelm- Gymnasium in Höxter mein Abitur mit einer Abschlussnote von 1,8. Während der Schulzeit verschaffte ich mir durch Praktika im Dentallabor Düker in Höxter und in der Zahnarztpraxis Sonja Richter in Fürstenau einen Einblick in den zahnärztlichen Tätigkeitsbereich. Bereits im Oktober 2004 konnte ich mit meinem Studium der Zahnmedizin an der Georg- August- Universität in Göttingen beginnen. Im Jahr 2005 schloss ich das Vorphysikum mit der Gesamtnote sehr gut ab. Zwei Jahre darauf bestand ich das Physikum mit der Note gut. Am 03. Dezember 2009 beendete ich das Studium der Zahnmedizin mit der Bewertung sehr gut im Staatsexamen. Die Approbation zur Zahnärztin erhielt ich am 08. Dezember 2009. Meine Zeit als Entlastungsassistentin leistete ich vom 01.01.2010 bis zum 31.12.2011 in der Gemeinschaftspraxis Dr. Thore Litta und Dr. Holger Quaas. Ab dem 01.01.2012 arbeitete ich mit Dr. Thore Litta und Dr. Holger Quaas als niedergelassene Zahnärztin in einer Berufsausübungsgemeinschaft (ehemals Gemeinschaftspraxis) zusammen. Nach dem plötzlichen Tod unseres Kollegen Dr. Thore Litta besteht die Gemeinschaftspraxis aktuell noch aus zwei Behandlern. Die Vorbereitungen zu meiner Dissertationsarbeit habe ich im Februar 2008 im Institut für Hygiene und Umweltmedizin in Göttingen unter der Leitung von Prof. Dr. Hartmut Dunkelberg aufgenommen. Nach dem Abschluss meiner Dissertationsarbeit strebe ich eine von der DGI (Deutsche Gesellschaft für Implantologie) anerkannte Fortbildung zur Implantologin an. Seit dem 06. April 2013 bin ich mit Hans- Jürgen Niemeyer (Chemielaborant) verheiratet und trage nun den Familiennamen Niemeyer (geborene Borgolte). 